Date of Publication: $17 / 6 / 2013$

\section{Cunninghamia}

A journal of plant ecology for eastern Australia
$\Leftrightarrow$ Domain Trust

ISSN $0727-9620$ (print) • ISSN 2200-405X (Online)

\title{
Revisiting Tony Price's (1979) account of the native vegetation of Duck River and Rookwood Cemetery, western Sydney
}

\author{
Alison Hewitt \\ Postgraduate student, School of Science and Health, University of Western Sydney, Hawkesbury campus, \\ Richmond NSW 2753, AUSTRALIA \\ email: a.hewitt@uws.edu.au
}

\begin{abstract}
The Duck River Reserve and Rookwood Cemetery in the highly urbanised Auburn district of western Sydney hold small but botanically valuable stands of remnant native vegetation. In the late 1970s, local resident G.A. (Tony) Price, recognised the value of these remnants, both for the species they held and the clues they could give us to the past, and spent three years surveying and collecting plants at these sites. Price recorded the species present and their abundance, and described the habitats in which they were found. He observed the ecology of plant interactions, moisture, shading and fire response, interpolating them into a picture of the landscape and vegetation of the district prior to European settlement. At a time when field botany was inaccessible to many, and the focus of conservation was largely on the broader scale, Price's local scale work at these sites was unusual and important. Though never formally published, Price's 1979 account 'The Vegetation of Duck River and Rookwood Cemetery, Auburn' has been cited in all subsequent work of consequence for the area. This paper presents and reviews Price's work and discusses his observations in relation to the current vegetation of these areas. Tony Price's contributions also highlight the value and role that ordinary citizens can play alongside professional botanists and plant ecologists in long term data collection, considered observation and environmental management. A copy of Price's original unpublished account has been included as an appendix to this paper.
\end{abstract}

Cunninghamia (2013) 13 :25-124

doi: 10.7751/cunninghamia.2013.13.002

\section{Introduction}

The Auburn district is approximately $18 \mathrm{~km}$ west of Sydney Harbour, on the eastern margin of the Cumberland Plain, that makes up the landscape of most of western Sydney. Agriculture and urbanisation in the 200 years since European arrival has resulted in the loss of an estimated $99 \%$ of the original native vegetation cover in the Auburn Local Government Area (NPWS 1997). Small remnants of native vegetation still survive in the perimeters of council parks and along railway corridors and creek banks. The largest stands are in Rookwood Cemetery and in the Duck River Reserve at South Granville where small, but botanically valuable, remnants of four communities listed as Critically Endangered
Ecological Communities under the NSW Threatened Species Conservation Act, 1995 (TSC Act) occur. These are Cooks River/ Castlereagh ironbark forest (CRCIF), Cumberland Plain woodland (CPW), Shale Gravel Transition forest, and Sydney Coastal River Flat Forest - Alluvial Woodland, lining the riverbank of the Duck River Reserve (species assemblages are described in the Final Determinations of the NSW Scientific Committee). The Duck River Reserve and Rookwood Cemetery also encompass important populations of two species listed as Vulnerable, (Acacia pubescens and Epacris purpurascens var. purpurascens) and two populations listed as Endangered (Pomaderris prunifolia and Wahlenbergia multicaulis) (Greening Australia 1990, Rookwood Plan of Management 1995). The two areas hold 
more than 20 floral taxa considered regionally significant because they are either rare or inadequately conserved in Western Sydney e.g., Asterolasia correifolia, Macrozamia spiralis and Eucalyptus viminalis at Duck River and Dillwynia parvifolia, Juncus procerus and Gonocarpus longifolius at Rookwood (Greening Australia 1990, NPWS 1997, James et al. 1999). The sites also provide habitat for listed vulnerable animal species such as the grey-headed flying fox, eastern bentwing bat, Cumberland land snail, regent honeyeater and green and golden bell frog. In addition, several bird species rare to the Sydney district, such as the zebra finch, yellowrumped thornbill, diamond fire-tail finch and double-barred finch; species that feed exclusively on grass seeds but require shrubby cover for nesting have been recorded (EDAW 1996, Smith \& Smith 1999, Applied Ecology 2011).

In the late 1970s, local Auburn resident G A (Tony) Price, recognised the value of these remnants both for the species they held and the clues they could give to past vegetation patterns, spent three years surveying and collecting plants in the area. He compiled an extensive list of the existing plant species, recorded ecological observations, and interpolated them into a picture of the landscape and vegetation of the district at the time of European settlement, in his unpublished account The Vegetation of Duck River and Rookwood Cemetery, Auburn. An electronic copy of Price's original account is included as Appendix 2 to this paper.

Greville Anthony (Tony) Price (1934-2010) grew up in Auburn and was a high school English/ History teacher and an avid collector of books. He gained his Master of Arts with $1^{\text {st }}$ Class Honours in History from the University of New England in 1964. He was also a student in the (then) School of Biological Sciences at Macquarie University in the 1980s. Aside from short teaching posts in Canberra and Armidale, Tony Price lived in the house on Park Rd, Berala that he owned and his parents owned before him. He died in April 2010 at age 75 (Kathleen Mealing, Laurence Gordon, Margot Wood pers. comm.).

Tony Price was widely consulted for his botanical knowledge, and his ecological work and opinions provided baselines for research, as well as environmental management by councils, catchment authorities, community groups and others, both locally and within the wider district. He was a founding member of the Friends of Duck River Reserve whose activities included: petitioning for interpretative signage; instigating fencing of the area to protect it from minibike riders and overflow parking at the Melita Soccer Stadium; gaining funding for the removal of old car bodies from the reserve; ensuring the retention of trees along the riverbanks; propagating local species and undertaking bush regeneration work.

Tony Price left a small bequest to Macquarie University to support future plant ecology research. This paper was supported by that bequest.

\section{Methods}

\section{Duck River and Rookwood site descriptions}

The Duck River Reserve covers 16 ha of Parramatta City Council Reserve $20 \mathrm{~km}$ west of Sydney's Central Business District on the western banks of Duck River, between Wellington and Everley Roads in South Granville (3352"04'S, 151 00" 48'E). Rookwood Cemetery lies 4km east between Lidcombe and Strathfield ( $33^{\circ} 52^{\prime}{ }^{\prime} 25^{\prime} \mathrm{S}, 151^{\circ}$ 03' $58^{\prime}$ E) covering 286 ha of Crown land under the control of the NSW Department of Lands, the majority intensively used as a cemetery. Conservation areas within Rookwood today total approximately 18 ha (DEM 2006).

Topography of the Auburn district is level to slightly undulating. Broad valleys and an alluvial floodplain drain the area that is $20-50 \mathrm{~m}$ above sea level, with gradients of less than three percent (Branagan et al. 1979, Parramatta River 9130-3N and Botany Bay 9130-3S 1: 25000 topographic maps). Duck River flows into the Parramatta River, draining the suburbs of Auburn, Berala and Birrong to the east, Sefton, Chester Hill, Granville and Clyde to the west and the northern part of Yagoona to the south. Eighty percent of river discharge is generated in wet weather flowing from impermeable urban surfaces and stormwater and carrying high nutrient loads and debris (EDAW 1996). The major drainage lines within Rookwood Cemetery are brick-lined canals (Price 1979).

The geology of the district comprises Ashfield and Bringelly shales of the Wianamatta Group, with a small sandstone outcrop at Duck River (Price 1979). The soils are yellow podsols, comprising silt and clay-sized alluvial materials, with additional areas of disturbed soils present (EDAW 1996). The area is classified as belonging to the Birrong Soil Landscape, characterised by soils that show seasonal waterlogging, low water permeability, low fertility, low water availability, low wet strength with hardsetting surfaces, and subsoils that are often saline (Chapman \& Murphy 1989). Soil depth varies but is typically $60 \mathrm{~mm}$ to clay and $1.8 \mathrm{~m}$ to shale (Glenn Piggott, pers. comm.).

The climate has mild, wet winters and warm, wet summers. Monthly average temperatures show summer maxima of $28.1^{\circ} \mathrm{C}$ in January, $17.2^{\circ} \mathrm{C}$ in July; and winter minima of $5.1^{\circ} \mathrm{C}$ in July, $18^{\circ} \mathrm{C}$ in January. Annual average rainfall is $983 \mathrm{~mm}$ (data from the Bureau of Meteorology, nearest rainfall records from Rookwood (Hawthorne Ave), nearest temperature records from Bankstown Airport).

\section{History}

Prior to European occupation, the Auburn district was occupied by the 'woods tribes' or Darug people. The Darug did not depend on fish and shellfish as the coastal indigenous peoples did; instead small animals and the tubers of native vines, lilies and orchids growing in the area comprised an important part of their diet (Granville Historical Society 1992, Kohen 1993). 
European settlers referred to the district around Auburn as 'Liberty Plains' with land granted to free settlers from 1793 (Auburn Municipal Council 1982). From the early 1800s, timber was extracted from the area - ironbark and turpentine trees for use in wharves and bridges; stringybarks for railway sleepers and roofing; and other local tree species for general firewood. Later, wattlebark was extracted for use in tanneries that operated along Duck River (Granville Historical Society 1992). As timber availability decreased, orchards and vineyards were tried but were unsuccessful, and the land was subsequently subdivided and sold for dairies, poultry farms and market gardens (Auburn Municipal Council 1982, Granville Historical Society 1992).

The Duck River Reserve is all that has survived from the original 600 acres granted to James Chisholm, a sergeant of the NSW Corps in 1823. It was grazed and sold on to a timber merchant in 1882 and then investors in 1885, before being purchased by Parramatta City Council in 1946 (Price 1979; Greening Australia 1990). Since this time, Parramatta City Council has maintained it as a public open space, while developing adjoining land as rubbish tips and a 'night soil' area, which more recently have become sporting fields and a golf course (EDAW 1996). By the end of the 1800s, there was much heavy industry operating along Duck River including Hudson's, Clyde Engineering and later the Commonwealth Engineering or Comeng site. Factory sites included flour mills, iron works, tool makers and several tanneries from 1877 (Granville Historical Society 1992).

In 1860 the Government Gazette newspaper carried an advertisement for ' 100 acres of land which may be suitable for a General Cemetery', setting out general requirements such as soil 'of considerable depth', 'drainage to an area whence water supply is not obtained for domestic purposes' and close proximity to 'the Great Southern Railway between Sydney and Parramatta' (Sigrist 1989). The government surveyor who subsequently visited the Auburn district reported that 'Cohen's Hyde Park Estate at Liberty Plains was covered with dense ti-tree and wattle scrub and wooded with mahogany, stringy bark and hollybutt, though the best timber had already been cut out' (Rookwood Plan of Management 1993). The land was purchased in lots in 1861 and 1864, and the first burial occurred at Rookwood in 1867. More land was purchased as burials increased in the decades that followed. Rookwood Necropolis is now the largest cemetery in the southern hemisphere (Rookwood Plan of Management 1993).

\section{Material and data sources}

Tony Price conducted his fieldwork in 1976, 1977 and 1978 and completed his written account in 1979. He states that he studied the Duck River site most intensively. $\mathrm{He}$ collected voucher specimens, utilised the limited number of published field guides that were available at that time and took specimens to the identification counter at the Royal Botanic Gardens in Sydney for assistance. He recorded abundance estimates on all species, coding them as common $(\mathrm{X})$, occasional $(\mathrm{O})$ or rare $(\mathrm{R})$ but did not record a decision rule as to how species were assigned into these abundance classes. He carefully recorded the habitat/s in which each species was found, dividing these into his eight 'microenvironments': 1. low woodland, Duck River; 2. ti-tree and eucalypt scrub; 3. grasslands; 4. exposed soils and subsoils; 5. drainage lines, edges of permanent sheets of water, creeks etc.; 6. permanent and transient sheets of water; 7. graves, Rookwood cemetery; and 8. dumped soil, edges of roads and tracks. Price was also interested in the ecological processes that were occurring in the vegetation and made notes on the apparent regeneration and establishment requirements of species: their light and shade tolerances and responses to waterlogging, drought, fire and soil disturbance.

Based largely on his surveys of the remnant native vegetation at Duck River and Rookwood Cemetery, Price reconstructed a picture of pre-European vegetation for the Auburn district. He also drew from his observations of the 'scattered, veteran eucalypts' of the State hospital grounds at Lidcombe, the Carnarvon Golf Course at Berala, and various parks and yards immediately east and west of these sites. He incorporated his knowledge of local land use and fire history and compared his conclusions on the pre-European vegetation with those postulated earlier by Pidgeon (1941) and Kartzoff (1969).

\section{Analysis}

An interpretation of Tony Price's 1979 account is presented, drawing from both his species list and written comments for details of vegetation structure, floristic and growth form assemblage, species richness and weed invasion at the sites. An examination of Price's species records was made, tallying richness and growth form and extracting records of species restricted within each of his eight microenvironments. Comparisons are made between the sites, to later studies at these and other western Sydney sites, and to the vegetation of these sites today. Sorensen's index was calculated as a similarity index between the two sites: Similarity $=2 a /(2 a+$

Table 1: Species and family richness (raw numbers with percentages in brackets)

Total Rookwood Duck River Unique to Rookwood Unique to Duck River Occurring both sites

$\begin{array}{lllllll}\text { Native species } & 311(53 \%) & 212(53 \%) & 254(54 \%) & 58 & 99 & 154(50 \%) \\ \text { Exotic species } & 273(47 \%) & 186(47 \%) & 213(46 \%) & 59 & 87 & 127(47 \%) \\ \text { All species } & 584 & 398(68 \%) & 467(80 \%) & 117(20 \%) & 186(32 \%) \\ \text { Families } & 110 & 89 & 99 & 11 & 21 & 781(48 \%)\end{array}$


$\mathrm{b}+\mathrm{c})$ where $\mathrm{a}=$ the number of species present at both sites, $\mathrm{b}$ $=$ the number of species present only at Rookwood, and $\mathrm{c}=$ the number of species present only at Duck River.

Price's species list (reproduced in Appendix 1) has been updated with plants listed alphabetically by current species name, within current family, under subheadings of growth form (derived from PlantNet and APNI websites). Species that Price recorded twice under two different names and are now considered the same species (taxonomic synonyms) have been listed only once (3 species) under their currently accepted name. Species missing both site and habitat codes (4 species) have been excluded. Species with missing or indecipherable site codes ( 2 species) or habitat recordings (35 species) have been included but location data left blank. The 35 taxa without habitat codes could not be used in habitat analyses. A number of other minor amendments, such as correcting spelling errors, or the erroneous coding of native species as weeds (or vice versa) were made.

\section{Results}

Price recorded a total of 584 species across the two sites: 311 native and 273 exotic (Table 1 ). The most species rich families were the Poaceae (88 species), Myrtaceae (61 species), Asteraceae (49), Fabaceae subfamily Faboideae (46) and subfamily Mimosoideae (16), and Iridaceae (16). In terms of both native and exotic species Duck River was floristically more diverse than Rookwood. A higher number of plant families were also found at Duck River.

The proportions of native and exotic species are remarkably consistent at the two sites (53-54\% native, $46-47 \%$ exotic). $50 \%$ of the native species and $47 \%$ of exotics occurred at both sites. Sorensen's similarity index for the two sites was 0.64 for all species, 0.66 for native flora and 0.63 for exotic flora.

The proportions of the nine growth forms were also remarkably similar at the two sites (Table 2), as was the ranking of the growth forms based on the numbers of species within each (Figure 1). Herbs were the most numerous growth form, comprising 37-39\% of species. Graminoids (grasses, sedges and rushes) were the next most numerous, comprising $\sim 21 \%$ of species. Shrubs followed at 16-19\%, trees $12-15 \%$, subshrubs and climbers, each made up 4-5\% of species recorded, with ferns, succulents and aquatics in lesser numbers and each comprising $<1 \%$ of growth forms recorded. Duck River had more native and exotic herb, graminoid, shrub and climber growth forms. Rookwood had slightly higher numbers of native and exotic tree species overall, while subshrubs, aquatics and succulents occurred in similar numbers at the two sites (Table 2).

Of the 8 microenvironments, ti-tree and eucalypt scrub was the most species-rich (230 species) and a high percentage of those species $(78 \%)$ were natives (Figure 2, Table 3). Grasslands had the second highest number of species recorded $(n=209)$ but a smaller percentage were native $(61 \%)$. The drainage lines, creeks and edges of water also held high numbers of species, $(n=195)$ but a higher percentage of those were exotic (58\%). A large number of species were recorded
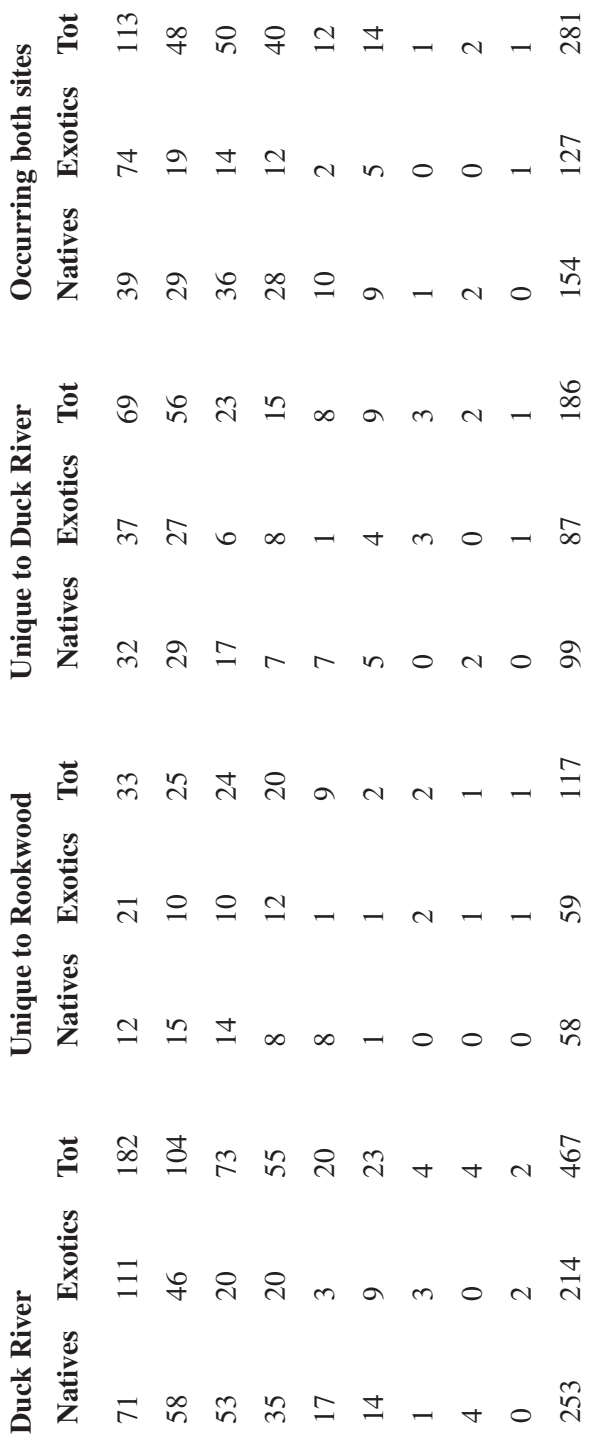

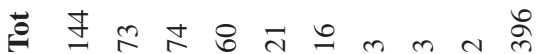

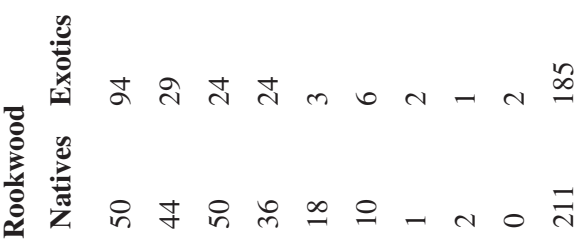

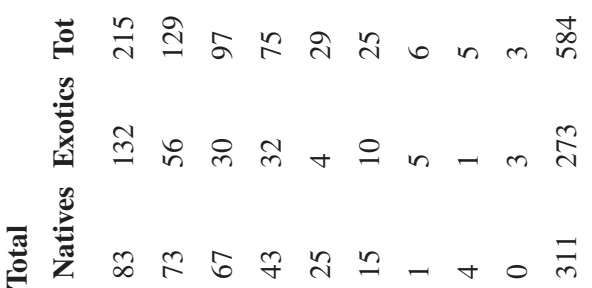

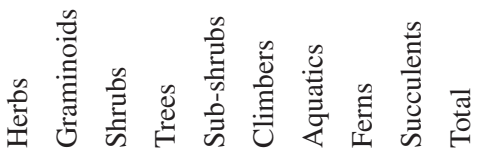




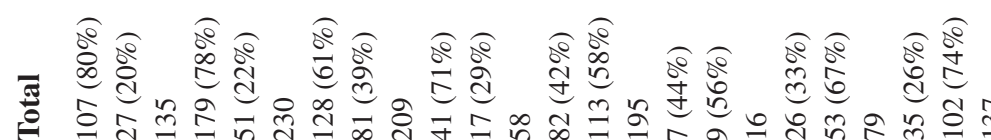
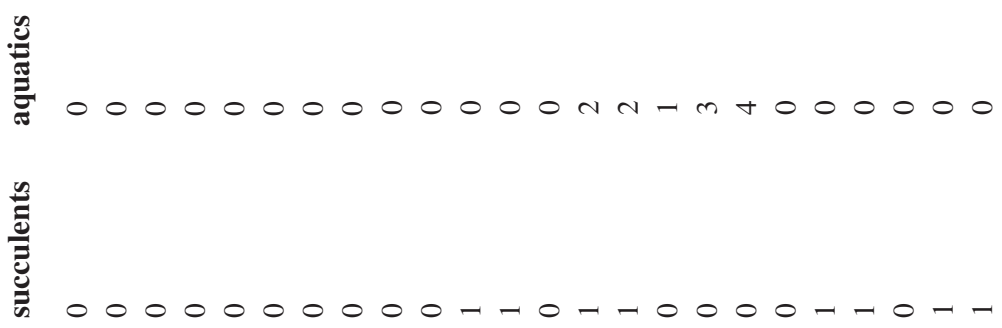

竞

$404-0-7.0-0004 h+000-0-000$

茜

ป

:

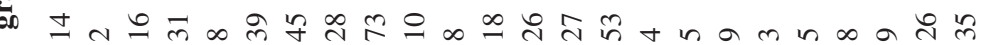

$\frac{n}{\frac{1}{n}}$

$n-\infty \stackrel{n}{n}=0$ J momn-mo0 $-n$ nnm n

章

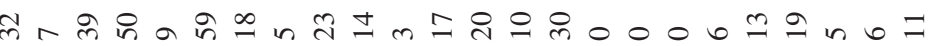

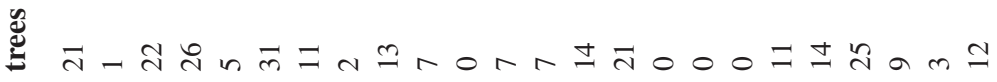

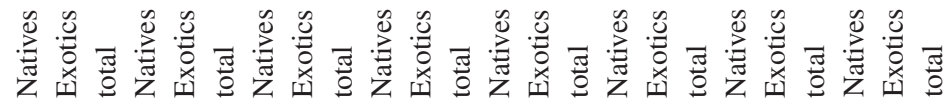

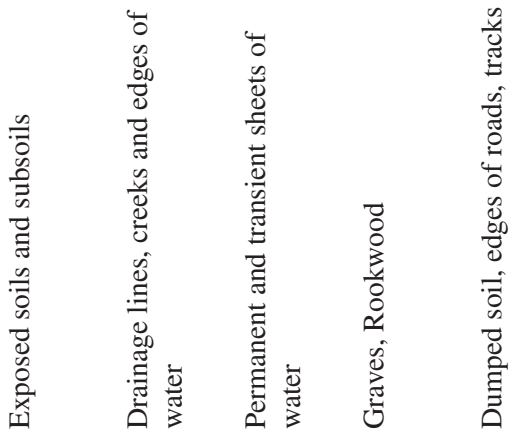




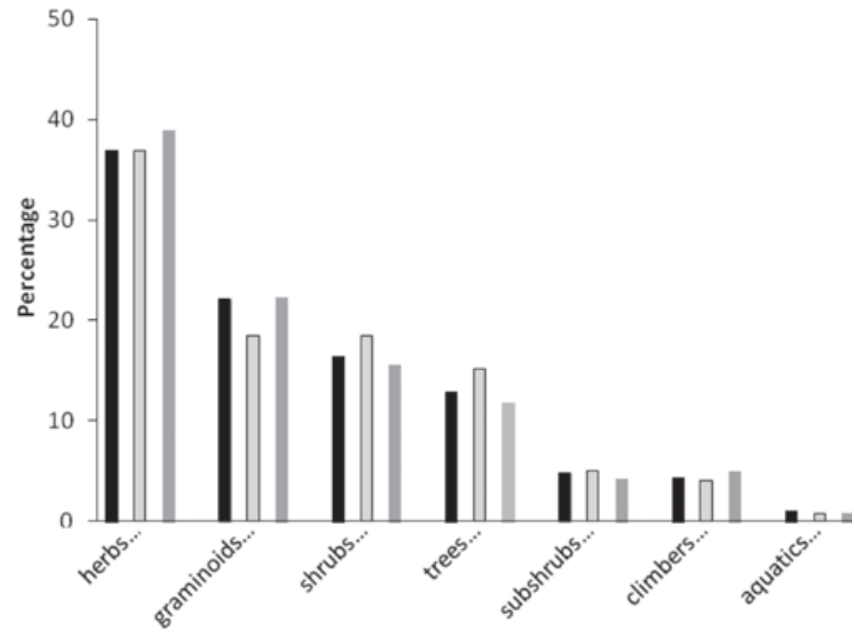

Fig. 1. Proportions of the 9 growth forms derived from Price (1979) as recorded at both sites (total), at Rookwood $\square$ and Duck River .

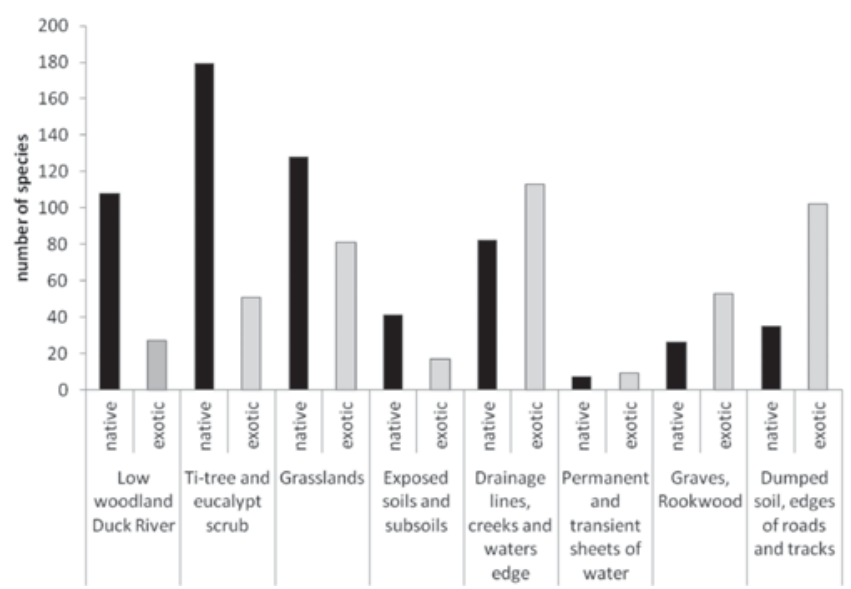

Fig. 2. The number of native and exotic species recorded by Price (1979) in each of his eight 'microenvironments'.

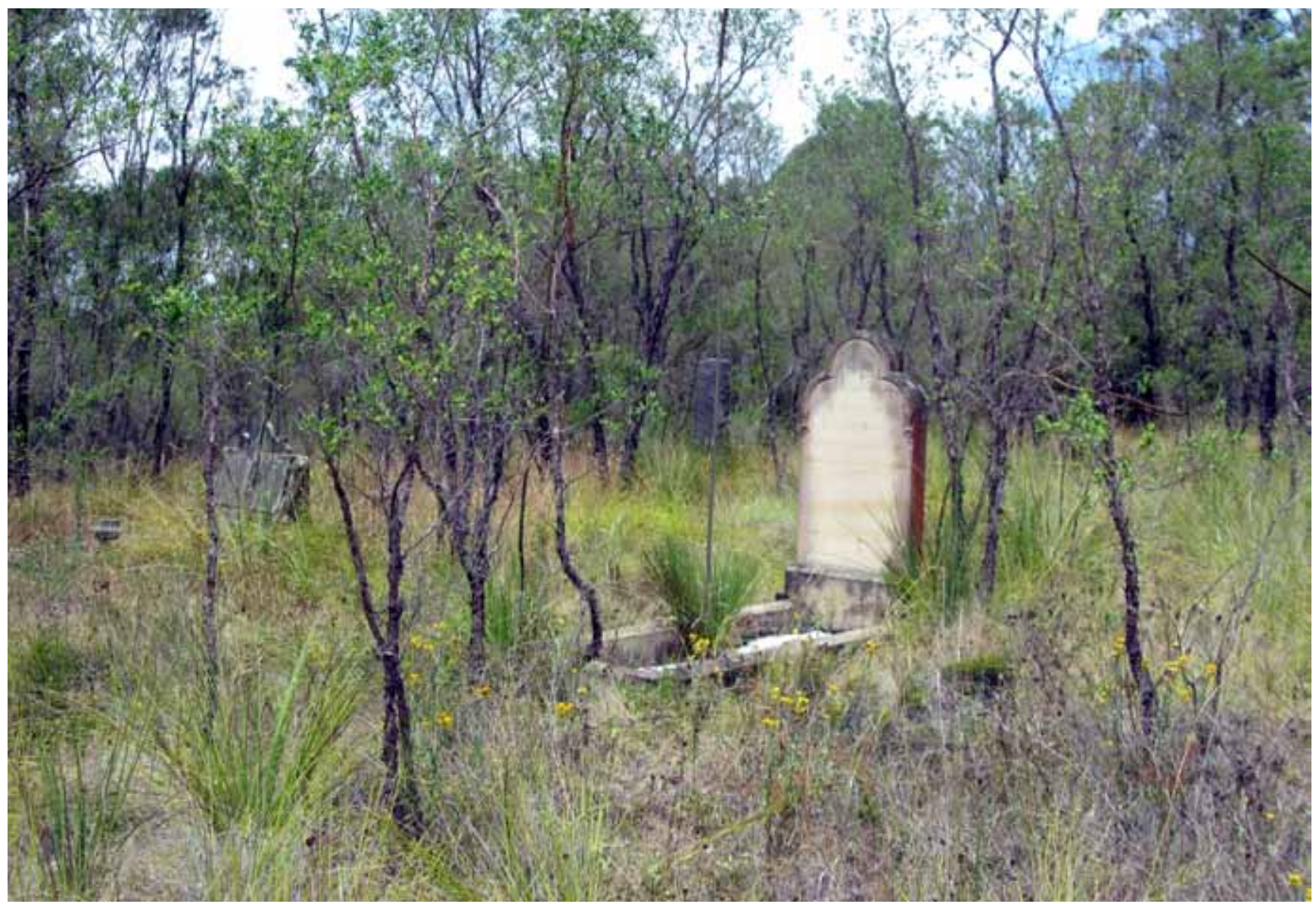

Fig. 3. Rookwood regeneration over graves: Some older sections of the cemetery are being managed to allow native plant regeneration with species present in this area including Dillwynia parvifolia, Daviesia ulicifolia, Xanthorrhoea resinosa, Macrozamia spiralis, Coronidium scorpioides, Stylidium graminifolium, Chrysocephalum apiculatum, Lissanthe strigosa, Angophora bakeri, Themeda australis, Patersonia longifolia, Pultenaea villosa, Hibbertia aspera, Leptospermum trinervium, Bursaria spinosa, Leucopogon juniperinus, Lomandra gracilis, Lepidosperma laterale, Hakea sericea seen here growing amongst the headstones. 


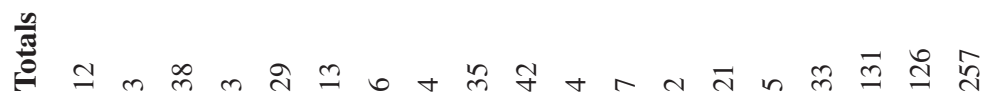

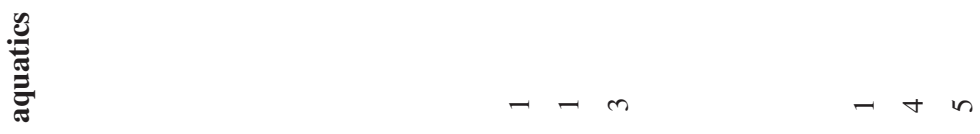

ڤ⿱艹

$0 \mathrm{n} n$

$\stackrel{\mathscr{E}}{\underline{E}}$

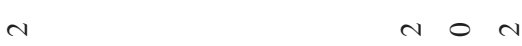

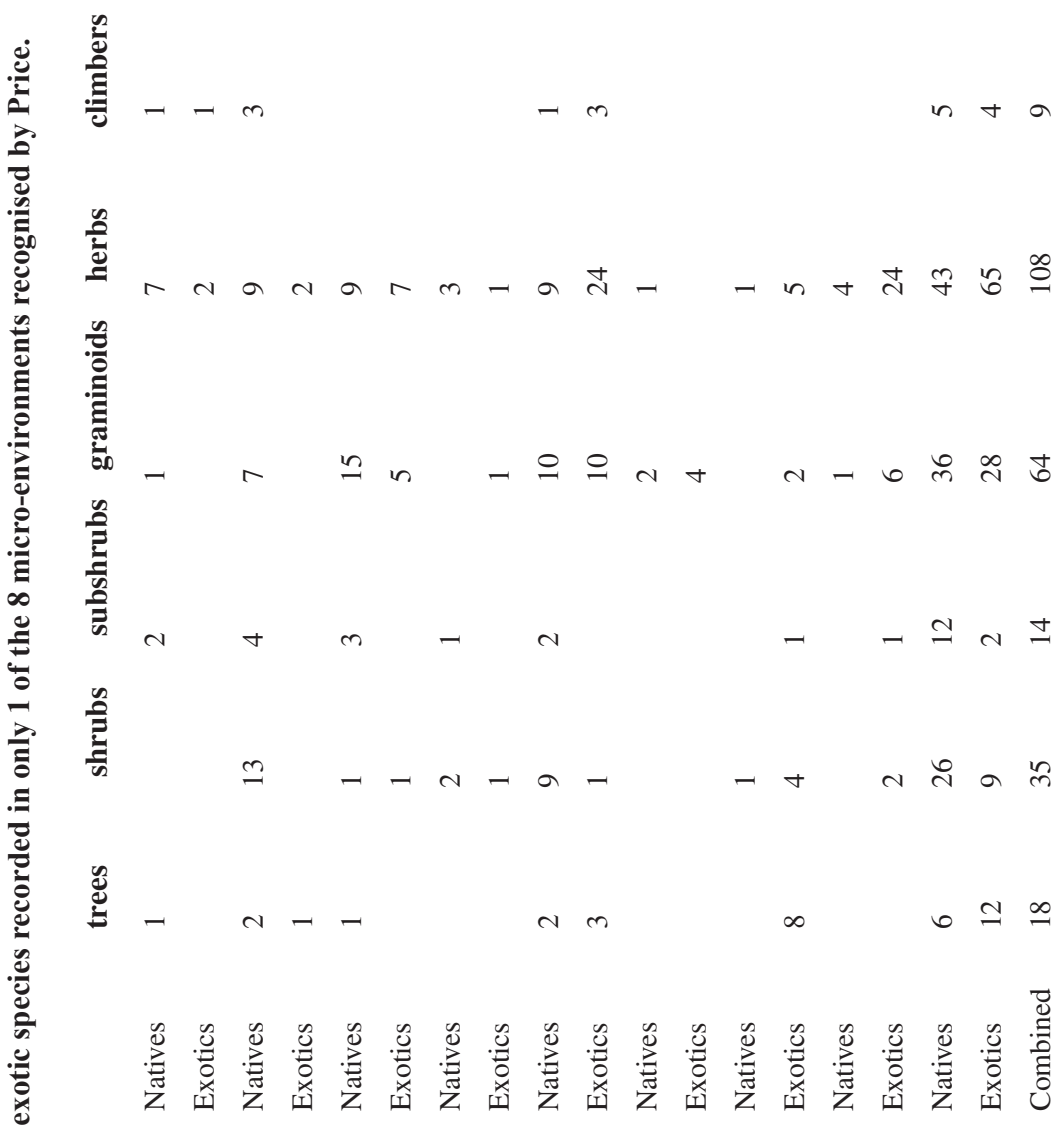




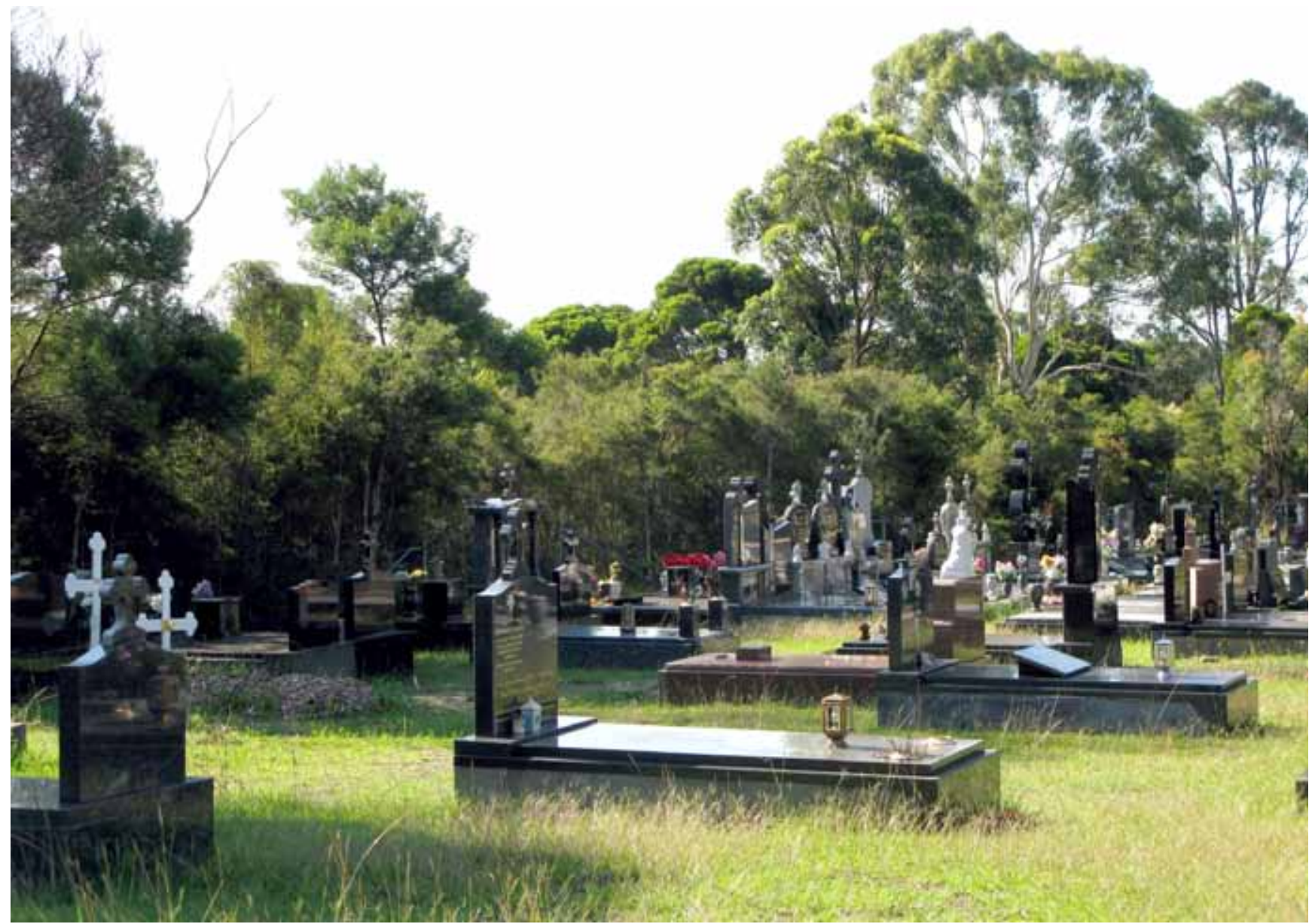

Fig. 4. Recent Rookwood graves in the foreground to one of the areas Tony Price termed 'ti-tree and eucalypt scrub' now a designated conservation area within the cemetery. Scattered Eucalyptus resinifera, Eucalyptus tereticornis, Eucalyptus sclerophylla, Angophora bakeri and Angophora floribunda overtop Melaleuca nodosa and Melaleuca decora with its striking mistletoe Amyema gaudichaudii. More unusual species in this area include Rhytidosporum procumbens, Hibbertia pedunculata, Dillwynia tenuifolia, Melaleuca erubescens, Bossiaea prostrata, Pultenaea retusa, Xanthorrhoea resinosa, Banksia spinulosa and Banksia oblongifolia.

from dumped soil, edges of roads and tracks $(n=137)$, with many of those being exotic $(74 \%)$. The low woodland at Duck River held a similar number of species $(n=134)$, but conversely showed the highest percentage of native species $(80 \%)$.

A total of 257 species (131 native and 126 exotic) were recorded in only one of the eight micro-environments recognised by Price (Table 4a). From an overall total of 584 species, this represents $44 \%$ of all species, being generally more restricted or rarer in occurrence. Of note are the 38 native species, of which 13 are shrubs, recorded only in the ti-tree and eucalypt scrub; the 29 natives (15 graminoids) recorded only in the grasslands; the 42 exotic species restricted to the drainage lines ( 24 of them exotic herbs) and 33 exotic species (with a further 24 exotic herbs) restricted to the disturbed soil environments, as well as an interesting suite of 21 exotic species restricted to graves (Table $4 \mathrm{~b}$ ).

In his 1979 account Price made numerous observations on individual species, their regeneration and establishment requirements, light and shade tolerances and responses to waterlogging, drought, fire and soil disturbance. He also recorded flowering and fruiting times for the dominant eucalypts. Many of these observations have been subsequently incorporated into the 'Ecology of Sydney Plant Species' series by Benson and McDougall in Cunninghamia (1993-2005). Readers can go to this source for more comprehensive information on the ecology of individual plant species.

\section{Discussion}

Tony Price's account raises a wide range of issues that are relevant to the conservation and management of remnant bushland in an urban context today. In light of current plant ecological understanding and the vegetation at the sites today I review Price's work below under six headings: a) his picture of the vegetation of the Auburn district as it was prior to European settlement; b) his insights into the ongoing vegetation dynamics occurring at these sites in relation particularly to fire, moisture and light; c) his work as it informs 
Table 4b: Species recorded in only 1 of the 8 microenvironments, arranged by growth form. Exotics are indicated by an asterisk.

\section{Species restricted to low woodland, Duck River:}

\begin{tabular}{|c|c|}
\hline Trees: & Glochidion ferdinandi \\
\hline Subshrubs: & Boronia polygalifolia, Pomax umbellata \\
\hline Graminoids: & Juncus homalocaulis \\
\hline Herbs: & $\begin{array}{l}\text { Brunoniella pumilio, Brunoniella australis, } \\
\text { Einadia polygonoides, Brachyscome linearifolia, } \\
\text { Cardamine lilacina, Scaevola albida, } \\
\text { Lagenophora stipitata, *Aptenia cordifolia, } \\
\text { *Polycarpon tetraphyllum }\end{array}$ \\
\hline
\end{tabular}

Species restricted to ti-tree and eucalypt scrub:

Trees: $\quad$ Allocasuarina littoralis, Eucalyptus sideroxylon, $*$ Morus nigra

Shrubs: $\quad$ Isopogon anemonifolius, Persoonia laurina, Pomaderris prunifolia, Exocarpos strictus, Banksia spinulosa var. spinulosa, Epacris purpurascens var. purpurascens, Melaleuca erubescens, Podolobium ilicifolium, Monotoca scoparia, Acacia stricta, Acacia suaveolens, Notolaea ovata, Banksia oblongifolia

Subshrubs: Hibbertia diffusa, Gompholobium minus, Opercularia aspera, Micrantheum ericoides

Graminoids: Gahnia melanocarpa, Digitaria parviflora, Dianella revoluta, Lomandra multiflora subsp. multiflora, Lomandra longifolia, Austrodanthonia racemosa var. racemosa, Paspalidium aversum

Herbs:

Einadia nutans subsp. linifolia,

Senecio quadridentatus, Senecio linearifolius, Solenogyne bellioides, Vittadinia dissecta, Wahlenbergia stricta, Goodenia heterophylla, Orthoceras strictum, Pterostylis nutans, *Chenopodium album, *Tropaeolum majus,

Climbers: Billardiera scandens var. scandens, Cassytha glabella, Cassytha pubescens

\section{Species restricted to grasslands:}

Trees: Melaleuca quinquenervia

Shrubs: Melaleuca armillaris, *Nerium oleander

Subshrubs: Bossiaea buxifolia, Gompholobium glabratum, Gonocarpus longifolius

Graminoids:

Carex inversa, Eragrostis leptostachya, Sporobolus elongatus, Austrodanthonia setacea, Lomandra cylindrica, Lomandra fluviatilis, Lomandra gracilis, Dichelachne rara, Austrostipa mollis, Aristida ramosa, Eriochloa pseudoacrotricha, Panicum simile, Sorghum leiocladum, Juncus procerus, Juncus sarophorus, *Dactylis glomerata, *Phalaris minor, *Poa pratensis, *Briza subaristata, *Chloris gayana
Herbs: $\quad$ Calotis lappulacea, Senecio pinnatifolius var. pinnatifolius, Linum marginale, Caesia parviflora, Caesia parviflora var. vittata, Zornia dyctiocarpa, Goodenia bellidifolia, Plantago varia, Hypoxis hygrometrica, , *Cerastium glomeratum *Lotus subbiflorus, *Richardia stellaris, *Misopates orontium, *Sparaxis spp., *Silene gallica var. gallica, *Romulea longifolia

Species restricted to exposed soils and subsoils:

Shrubs: Acacia longissima, Kunzea ambigua, *Lycium ferocissimum

Subshrubs: Astroloma humifusum

Graminoids: *Ehrharta longiflora

Herbs: Dysphania littoralis, Vittadinia muelleri, Chamaesyce drummondii, *Hypochaeris microcephala

Succulents: *Aloe spp.

Species restricted to drainage lines, creeks:

Trees: $\quad$ Melaleuca linariifolia, Casuarina glauca, *Salix babylonica, *Prunus domestica, *Prunus persica,

Shrubs: Leucopogon lanceolatus var. lanceolatus, Leptospermum polygalifolium, Melaleuca ericifolia, Hovea longifolia, Goodenia ovata, Persoonia linearis, Viminaria juncea, Pomaderris lanigera, Asterolasia correifolia, *Ricinus communis

Subshrubs: Mirbelia rubiifolia, Opercularia varia

Graminoids: Juncus subsecundus, Juncus continuus, Schoenus apogon, Crinum pedunculatum, Cyperus mirus, Amphibromus neesii, Phragmites australis, Joycea pallida, Arundinella nepalensis, Urochloa foliosa, *Stenotaphrum secundatum, *Phormium tenax, *Bromus molliformis, *Bromus rubens, *Cortaderia selloana, *Eragrostis curvula, *Holcus lanatus, *Axonopus fissifolius, *Pennisetum glaucum, *Setaria palmifolia,

Herbs: $\quad$ Centrolepis strigosa, Centipeda minima, Epilobium billardierianum subsp. cinereum, Einadia trigonos, Isotoma fluviatilis, Goodenia paniculata, Persicaria lapathifolia, Persicaria decipiens, Solanum americanum, *Zantedeschia aethiopica, *Spergularia rubra, *Stellaria media, *Bidens subalterans, *Apium graveolens, *Hydrocotyle bonariensis, *Leucojum aestivum, *Canna indica, *Canna spp., *Brassica fruticulosa, *Brassica juncea,

*Brassica rapa subsp. sylvestris,

*Rorippa nasturtium-aquaticum, *Fumaria muralis, *Mentha x. piperita, *Hippeastrum puniceum, *Gladiolus cuspidatus, *Linum usitatissimum, *Mirabilis jalapa, *Polygonum arenastrum, *Plantago coronopus, *Artemisia vulgaris, *Cotula coronopifolia, *Vellereophyton dealbatum, 
Ferns: $\quad$ Pellaea falcata, Nephrolepis cordifolia

Climbers: $\quad$ Parsonsia straminea, *Anredera cordifolia, *Lonicera japonica, *Cardiospermum grandiflorum

Aquatics: $\quad$ *Sagittaria platyphylla

Species restricted to permanent and transient sheets of water:

Graminoids: Isolepis inundata, Paspalum distichum, *Isolepis prolifera, *Juncus capitatus, *Scirpus chlorostachys, *Polypogon monspeliensis

Herbs: Alternanthera denticulata

Aquatics:

Typha orientalis, *Myriophyllum aquaticum, *Eichhornia crassipes, *Alternanthera philoxeroides

Species restricted to graves:

Trees: *Pinus halepensis, *Pinus pinaster, *Pinus pinea, *Pinus radiata, *Tristania conferta,

*Eucalyptus melliodora, *Araucaria bidwillii, *Robinia pseudoacacia

Shrubs: Acacia myrtifolia, *Ulex europaeus, *Polygala myrtifolia, *Rhaphiolepis indica, *Lantana montevidensis

Subshrubs: *Pelargonium asperum

Graminoids: *Paspalum urvillei, *Crocosmia $x$. crocosmiiflora

Herbs: Diuris punctata, *Vinca major, *Lavandula stoechas, *Oxalis purpurea, *Allium neapolitanum, *Watsonia aletroides

Succulents: *Agave americana

Species restricted to dumped soil, edges of roads, tracks:

Shrubs: $\quad *$ Genista linifolia, *Acacia podalyriifolia

Subshrubs: $\quad *$ Solanum linnaeanum

Graminoids: Juncus vaginatus, *Agapanthus praecox subsp. orientalis, *Triticum aestivum, *Chloris virgata, *Avena fatua, *Avena ludoviciana, *Avena sterilis,

Herbs: Suaeda australis, Calotis cuneifolia, Crassula sieberiana, Triptilodiscus pygmaeus, *Iris germanica, *Amaranthus hybridus, *Paronychia brasiliana, *Soliva anthemifolia, *Soliva sessilis, *Capsella bursa-pastoris, *Coronopus didymus, *Rapistrum rugosum, *Centranthus ruber, *Myosotis sylvatica, *Cichorium intybus, *Silybum marianum, *Tragopogon porrifolius, *Sisymbrium officinale, *Sisymbrium orientale, *Alyssum maritima, *Euphorbia peplus, *Chamaesyce prostrata, *Oxalis debilis var. corymbosa, *Oxalis latifolia, *Papaver somniferum subsp. setigerum, *Lupinus spp., *Arctotheca calendula,

*Aster subulatus, our understanding of weed invasion and native persistence within a suburban matrix; d) some comparisons between the vegetation at the two sites; e) prior and subsequent surveys at the sites and the recording of rare species; and f) the current conservation status of the two sites.

\section{a) Pre-1788 vegetation of Duck River and Rookwood}

Benson et al. (1999) and Benson \& Howell (2002) have suggested sources of information that can give us a picture of the landscape and vegetation at the time of European settlement in Australia. These include historical firsthand descriptions from journals, early letters and reports; material from museum collections and other systematic data collections; old paintings and photographs; and an area's remnant vegetation in context with climate and geology. Price has used the latter to draw a picture of the area as tall woodland or forest with trees that were $60-80$ feet $(18-24 \mathrm{~m})$ high, with an understorey that was grassy, but prone to invasion by shrubs when fire-free intervals were longer. He described Duck River and Rookwood vegetation in 1979 as 'disturbed' from their original vegetation, noting that 'regrowth' and the 'scattered, veteran eucalypts' still standing pointed to past woodland or forest structure. He described the variety of shrub species in the understorey in 1979, noting the range of species 'from plants such as Kunzea ambigua and Pultenaea villosa that grow well only in the open, somewhat exposed situations, to others such as Glochidion ferdinandi, Breynia oblongifolia and Notelaea longifolia, which are normally plants of wet, shaded forests'. He also noted the occurrence of shrub species more typical of sandstone soils, including Asterolasia correifolia, Banksia spinulosa, Pultenaea retusa, Pomaderris lanigera and Pomaderris ferruginea. In summary, the native species were predominantly of a suite pertaining to clay based soils but with lesser numbers of species of a sandstone flora, particularly in Rookwood.

Price postulated that pre-1788 the area was dominated by Eucalyptus moluccana and Eucalyptus fibrosa while commenting on the diversity of tree species present at the sites, noting the presence in lower numbers of 'tree species from the drier west such as Eucalyptus eugenioides, Eucalyptus parramattensis and Eucalyptus longifolia' and that these 'mingle with others from wetter areas such as Eucalyptus resinifera and Syncarpia glomulifera'. Of the trees he notes also the presence of 'those that grow best near to waterholes', such as Angophora floribunda and Eucalyptus amplifolia (the latter only at Duck River) and to species more typical of sandy soils 'such as Angophora bakeri, Eucalyptus punctata and Eucalyptus sclerophylla'. Price disputed Kartzoff's (1969) idea of the area as one of blackbutt and Sydney blue gum (Eucalyptus pilularis - Eucalyptus saligna) high forest extending as far west as Merrylands and Granville. Rather, he agreed with Pidgeon's (1941) picture of assemblages of forest species typically found on clay shales that she recorded from the Bankstown-Liverpool district. Price's interpretation accords with Benson \& Howell's (1990a, b) and Tozer's (2003 \& 2010) reconstructions of the vegetation across the Cumberland Plain. 


\section{b) Vegetation dynamics - fire, moisture and light}

Price described the scenario of 'too-frequent fires' reducing species diversity at both sites, noting that species with longer primary juvenile periods need fire-free intervals long enough to be able to flower and fruit before a subsequent fire, so as not to 'exhaust the gene pools'. He noted the succession dynamics of the vegetation in response to fire frequency, drawing a picture of scrub or shrubby areas 'reverting' to eucalypt woodland in the absence of fire, while following fire these areas return to grassland, which in turn, is re-invaded by shrubs and trees if fire is long absent. His conclusions are consistent with current understanding of secondary succession dynamics and disturbance by fire, both locally in Cumberland Plain woodland (see for example, Watson et al. 2009) and at a global scale (see Bond et al. 2005).

Price noted a bias towards Melaleuca species at the expense of eucalypts under regimes of frequent fire. He also noted that tree species that were quicker to reach reproductive maturity, such as Angophora bakeri, Angophora floribunda, Eucalyptus eugenioides and Eucalyptus globoidea, or those that had fire resistant bark (Syncarpia glomulifera, Eucalyptus longifolia and Eucalyptus resinifera), were able to 'persist better' under frequent fire, compared to those he states were 'more fire sensitive'. He suggested frequent fire as the cause of the decline in numbers of the once dominant Eucalyptus moluccana, seen at Rookwood but not Duck River.

The regular cool hazard reduction grass fires that had previously occurred every 2 years across large areas of Rookwood Cemetery had become less frequent by the mid-1970s according to Price, owing to tightening of the Environment Protection Authority's air pollution regulations (Price 1979). In 2001 a large, uncontrolled fire occurred in the eastern conservation area (Cemetery Areas 8, 27 and 28) and occasional small arson fires are currently reported by grounds staff at Rookwood cemetery (Glenn Piggott, pers. comm). The Duck River Reserve was not subject to such regular, deliberate burning in its earlier years, and the greater species richness recorded there may reflect this. However, in the 1980s it was noted with concern that some areas of the Duck River Reserve were subject to spot fires as frequently as annually (Price, G.A. cited in Greening Australia 1990). Parramatta City Council (2012) reports that for decades the reserve has been subject to arson fires 'at least every 2 years somewhere in the reserve' (David Kuhle, pers. comm.).

Price made reference to the 'vigorous growth habits' of the Melaleuca species outcompeting other species for light, forming 'dense stands' and 'suppressing competitors', particularly noting Melaleuca nodosa, which he described as 'aggressive and tolerant'. Even today at these sites, species of Melaleuca, particularly Melaleuca nodosa, develop large seed loads and recruit continuously, with adequate moisture, to develop a mixture of age cohorts in the field. They are seen colonising bare areas, eventually forming dense thickets and casting shade that suppresses much recruitment beyond the seedling stage (Hewitt unpub. doctoral studies). Price (1979) wrote that 'few plants other than tough herbs... and a few tufts of tolerant grasses...' were able to '...linger on in the dense shade' (of the Melaleuca). Price described 'the struggle to reach the canopy' with competition for light, and the way in which eucalypts, in contrast to Melaleuca, 'open up' the canopy somewhat allowing in light. This concurs with Bale et al. (1998) who remark that eucalypts have an open canopy structure and leaves of a pendant habit, allowing more direct and scattered light into the subcanopy. It is because of these growth habits that Price, while cautioning against too frequent a fire regime, reported fire as 'necessary to keep the ti-tree in check'.

'Niche partitioning' according to light and moisture levels was evident to Price in the distribution of grass species at the two sites. He wrote: 'At the most exposed, seasonally driest end of the spectrum in grassy glades Themeda australis may be dominant; in moister areas of dappled shade Microlaena stipoides is most common; between the 2 extremes there tends to be a mixed stand with Microlanea stipoides, Entolasia marginata, Echinopogon ovata and Echinopogon caespitosus more frequent in sheltered sites while in exposed places Danthonia species, Aristida vagans, Stipa species, particularly Stipa nervosa, Dichelachne sciurea and Eragrostis brownii are more common'. He noted also native Agrostis species present at the moistest sites while 'Dichelachne sciurea, Eragrostis brownii and Aristida vagans are more successful at drier sites' (Price 1979). Price's idea of niche partitioning in the grassy layer, according to light and moisture levels, warrants further study, and has implications particularly for restoration efforts in grassy woodland communities.

\section{c) Exotic species}

The origin of the exotic species that Price recorded at the sites is interesting, with a number of those from Rookwood naturalised from cultural plantings at the site (e.g. 11 tree and palm species are restricted to graves). Exotic species used from the cemetery's inception to achieve a VictorianEdwardian gardenesque style included formal avenues of Phoenix canariensis, Pinus and Araucaria species. Other exotics now growing in Rookwood were probably once planted over graves for their religious or Victorian funereal symbolism. These include perfumed species like honeysuckle (Lonicera japonica) representing innocence or sweetness of disposition, climbers or vines like Chinese wisteria (Wisteria sinensis) and morning glory (Ipomoea indica) to signify the bonds of love, roses for sinlessness, lilies for purity, and the varied palms and cedars for their biblical and quaranic connotations (Burke \& Betteridge 1989). Pinus and Cupressus, 'evergreen' species, have symbolised the afterlife or life everlasting since Roman times. Many of these species now fall into the category of major weeds within the cemetery. UBM (2011) list problematic weeds within the cemetery as hawthorn (Raphiolepis indica) and privet (Ligustrum sinense), both of which were planted as hedges in the cemetery from the 1870s; Coreopsis lanceolata, Watsonia meriana 'Bulbilifera' (widely used as grave planting in the late 1800s), Camphor laurel (Cinnamomum camphora), freesia, bridal creeper (Asparagus asparagoides), Crofton weed (Ageratina adenophora), blue periwinkle (Vinca major), 


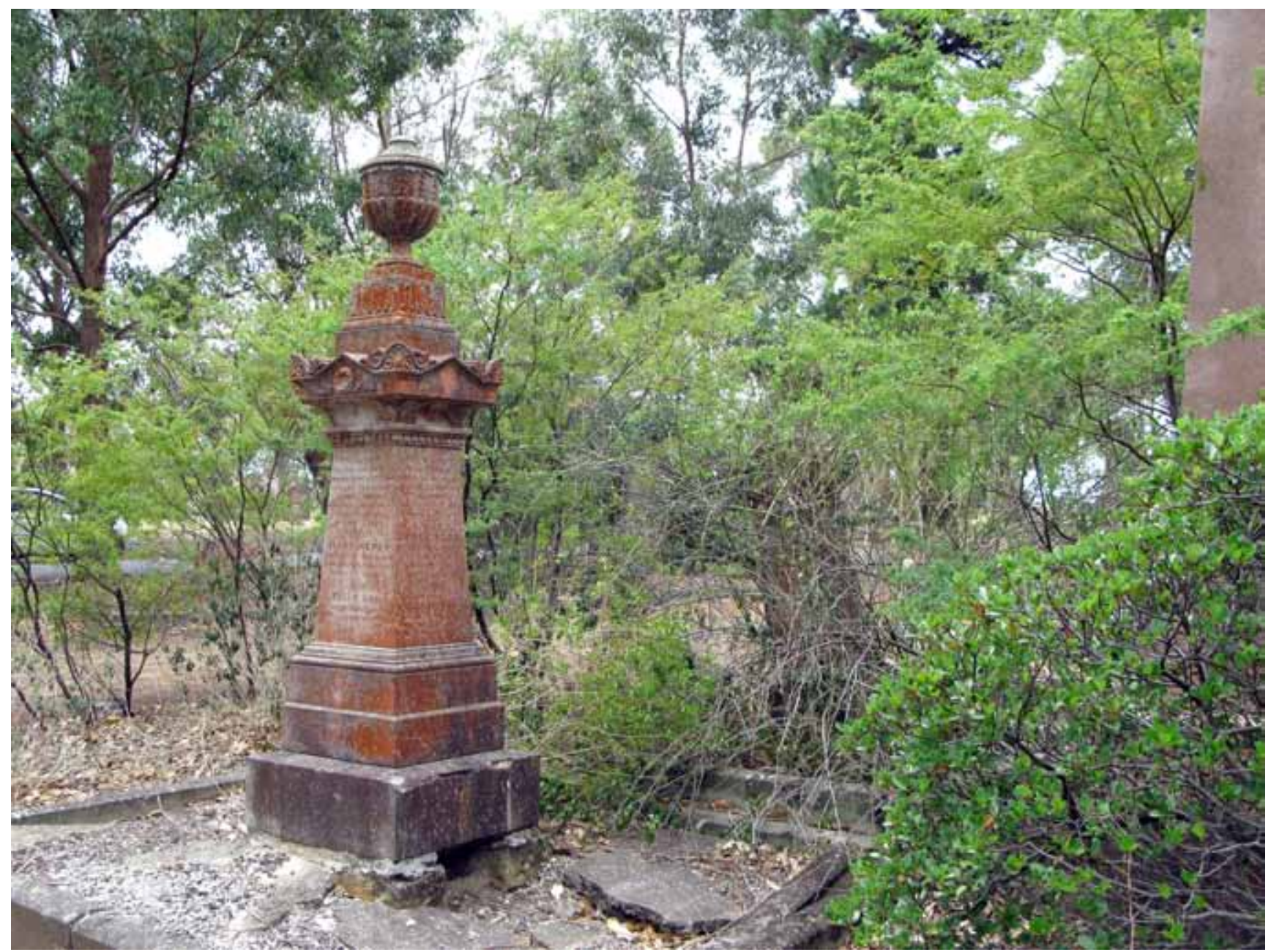

Fig. 5. The endangered species Acacia pubescens is seen here regenerating at Rookwood amongst graves and exotic species (Rhaphiolepis indica to the right).

lantana (Lantana camara and Lantana montevidensis), boneseed (Chrysanthemoides monilifera subsp. monilifera), blackberry (Rubus spp.), castor oil plant (Ricinus communis), pampas grass (Cortaderia selloana), prickly pear (Opuntia stricta) and water hyacinth (Eichhornia crassipes).

Some Eucalypts from other parts of Australia have naturalised at Rookwood following planting of a few individuals e.g., Eucalyptus saligna (Sydney Blue Gum), Eucalyptus punctata (Grey Gum), Eucalyptus melliodora (Yellow Box), Eucalyptus microcorys (Tallowwood) and Eucalyptus citriodora (Lemon-scented Gum).

Some of the exotic tree species Price recorded at Duck River may also originate from deliberate plantings, with some traced to the time immediately following Parramatta City Council's acquisition of the land in 1946, e.g., Erythrina sykesii (Indian coral tree), Salix babylonica (weeping willow), Populus nigrus (poplar) and Cinnamomum camphora (camphor laurel) (EDAW 1996).

At the time of Price's work many of the exotic species were only present in drainage lines, on dumped soil and on the edges of roads and tracks (Tables $4 a$ and $4 b$ ). This is a picture commonly seen in urban bushland such as the Duck River and Rookwood sites today, where run-off increases soil nutrients and moisture levels, favouring the growth of weeds (see discussion in Benson \& Howell 1990a). By contrast, the areas with the lowest percentage of exotics (Tables 3 and $4 a$ ) are the core bushland areas Price termed Duck River woodland and Ti-tree and eucalypt scrub. This, too, is in accordance with what we know about the resilience of unploughed stands of native vegetation and priority effects. In assessing the impact of clearing and grazing history on the species composition of Cumberland Plain woodland remnants, Hill et al. (2005) found that clearing with soil disturbance had the highest impact on native species decline and exotic invasion, with grazing alone not dramatically impacting on composition. Price expressed it more colloquially from his own observations (page 10): 'Most of the original species no doubt still occur as clearing with axe and fire without years of laborious grubbing and weeding is somewhat akin to sowing dragon's teeth. The native species able to regenerate from rootstocks, lignotubers and stumps....'.

The abundance and microenvironment data for the exotic species that Price recorded in the 1970 s provides a 


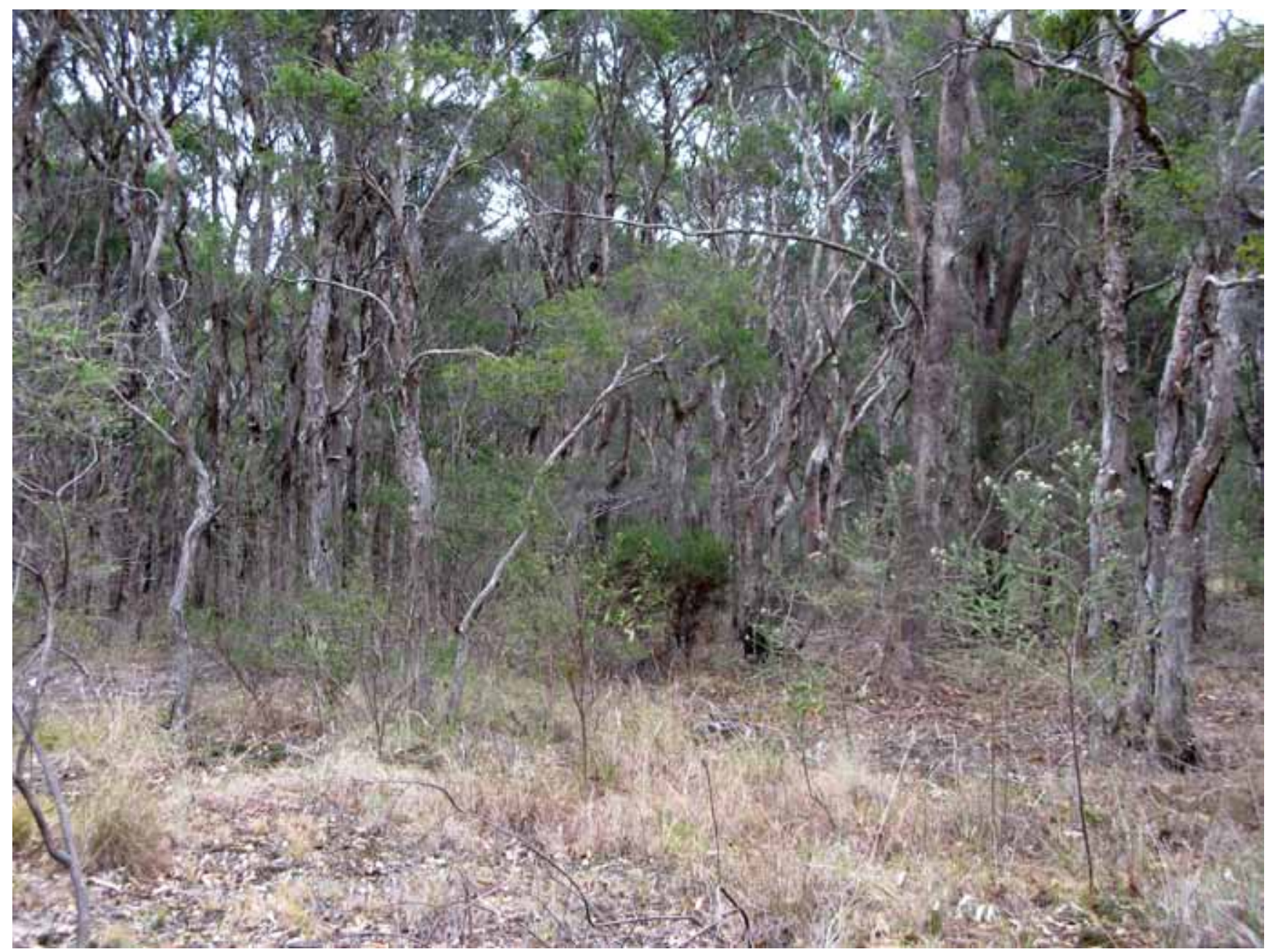

Fig. 6. A species rich woodland understorey at Duck River Reserve in South Granville where Tony Price recorded many native species. In Summer the pretty green flowers of Callistemon pinifolius and the dark blue fruits of Polyscias sambucifolia can be seen in the shrub layer. Diverse native groundcovers and herbs include Brunoniella australis, Bossiaea prostrata, Vittadinia muelleri, Dichondra repens, Glycine clandestina, Desmodium varians, Polymeria calycina, Caesia parviflora, and Astroloma humifusum.

comparison with the present. For example, he recorded Vinca major as only occasional at Rookwood over graves, and Rhaphiolepis indica as rare; both are now considered common and problematic weeds across the site. The major weeds today include a number of significant ones not recorded by Price, including (at Rookwood) Andropogon virginicus (Whisky grass), Olea europaea subsp. cuspidata (African olive), Eragrostis curvula (African lovegrass), Hypericum perforatum (St. John's wort), Cytisus scoparius (English broom) and Grevillea robusta. At Duck River, Thunbergia alata (Black-eyed Susan), Passiflora edulis (Common passionfruit), Ipomoea cairica (Coastal Morning Glory) and Acetosa sagittata (Rambling Dock) are additional exotic vines along the riverbanks. Olea europea and Eragrostis curvula in particular have established widely elsewhere in western Sydney since the 1970s (Doug Benson pers. comm. 2012) and indicate the short time spans over which invasion and vegetation change can occur.

\section{d) Differences between Rookwood and Duck River}

Price noted that a number of ground orchids (Diuris, Microtis and Thelymitra) associated particularly with the Themeda grasslands at Rookwood were not present at Duck River. He suggested that these species had survived at Rookwood and not Duck River, due to different landuse histories, Rookwood with its many decades of protection from stock grazing while still exposed to frequent burning. Native geophytes of grassy woodlands are now rare, although frequently found in cemeteries where they are not at risk from stockgrazing, but still vulnerable to lawnmowers, herbicides and 'tidying up' (Loneragan 1975, Semple-Kerr 1985, McBarron et al. 1987, Barrett \& Barrett 2001). Sadly, the area of Themeda grassland that Price noted as rich in native Liliaceae and Orchidaceae (he mapped it later in 1993) has since disappeared under a monoculture of kikuyu (Pennisetum clandestinum) and the neatly rowed headstones of a lawn cemetery. 
Price noted the many species in common to both sites, but pointed to higher numbers of naturalised exotics and a suite of native species more commonly found on sandstone substrates, that were collected from Rookwood. Sorensen's index of similarity shows a moderate degree of similarity in species assemblage between the two sites; 0.649 or $65 \%$ for all species, 0.662 or $66 \%$ for native flora and 0.635 or $64 \%$ for exotic flora. By comparison, Analyses of Similarity of quadrat data collected from seven remnant Cumberland Plain Woodland sites across western Sydney (French et al. 2000) showed similarities of 25-49\% (Bray-Curtis dissimilarity scores of 51-75\%). The lower similarity amongst these sites compared with the higher proportion of species shared at Duck River and Rookwood in the late 1970s might have a number of causes. Possibly the sites assessed by French et al. (2000) are of different sizes, with different edge effects and different propagule pressures; or that they have been subject to different disturbance factors; or that the intervening decades between Price's surveys and this later work has enabled greater divergence in species composition to have occurred. It would be worthwhile to recalculate similarity indices at Duck River and Rookwood today. Such data may even inform our understanding of species persistence traits against historical filtering by a range of disturbance types.
Most of the difference in species assemblage between Rookwood and Duck River is in the herb, shrub and graminoid growth forms (consistent with the French et al (2000) finding that differences between Cumberland Plain remnant sites were mainly due to differences in understorey species). The Rookwood/ Duck River comparison is unusual, (as noted by Price) in the higher number of exotic tree species at Rookwood, having naturalised from deliberate grave plantings.

Benson and Howell (2002) draw a picture from varied historical sources of Cumberland Plain woodland species assemblages being fairly continuous before European colonisation. Without major geographical boundaries to divide the plain, species would have been distributed reasonably uniformly. This they contrast with coastal rainforest communities that are naturally dissected (by soil and landscape features), resulting in species compositions that can vary markedly from site to site, with many isolated species occurrences. This latter type of variation in species occurrences is more in accordance with the picture Price recorded at Rookwood and Duck River and of the work of French et al. (2000), and is consistent with the species compositions given by Benson and Howell (2002) of Cumberland Plain remnants today. The local distribution and frequencies of species has changed and each remnant can

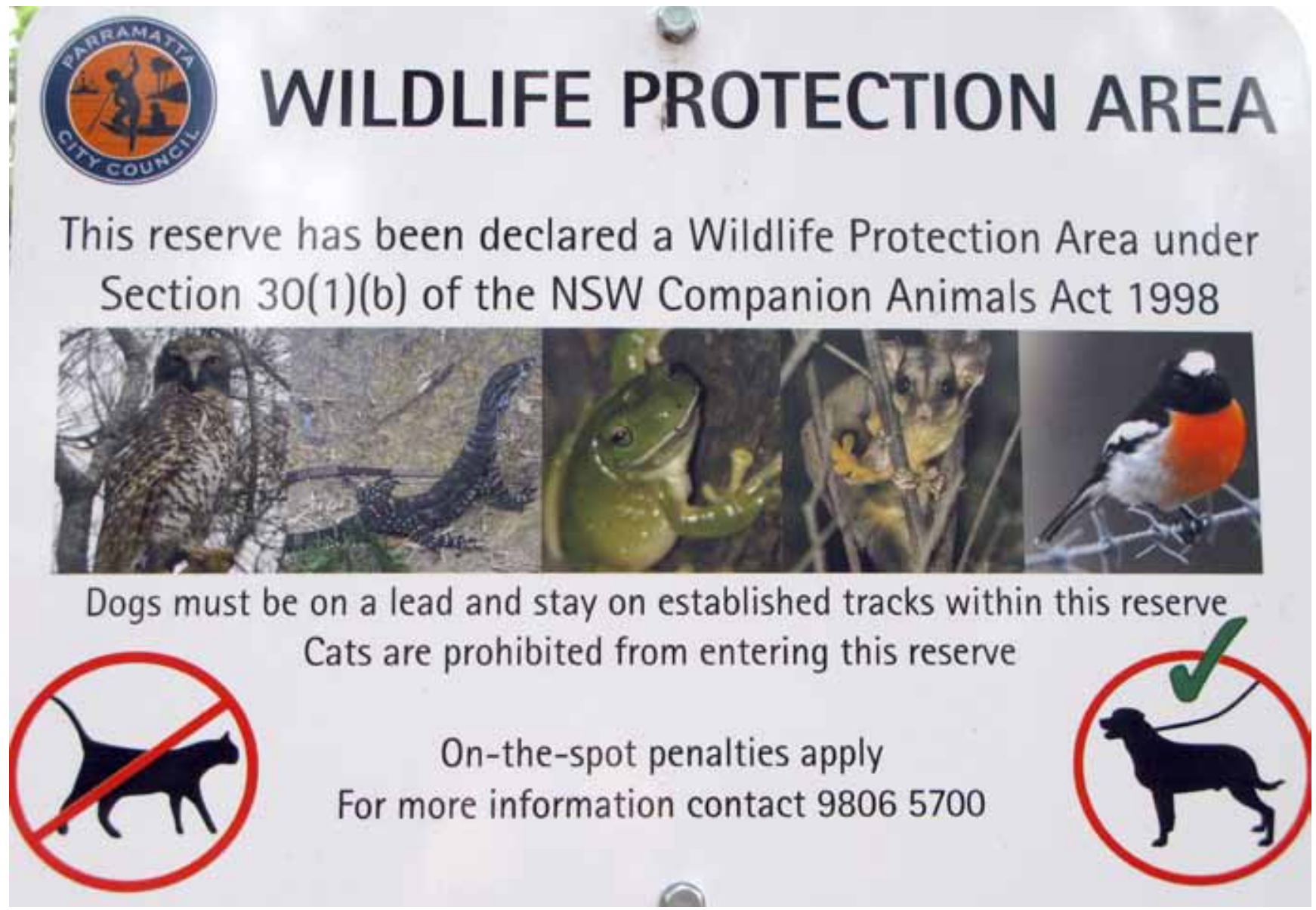

Fig.. 7. Parramatta City Council and the community have a strong commitment to caring for the woodland at Duck River and council recently declared the Duck River Reserve a Wildlife Protection Area prohibiting cats or dogs off leashes. 


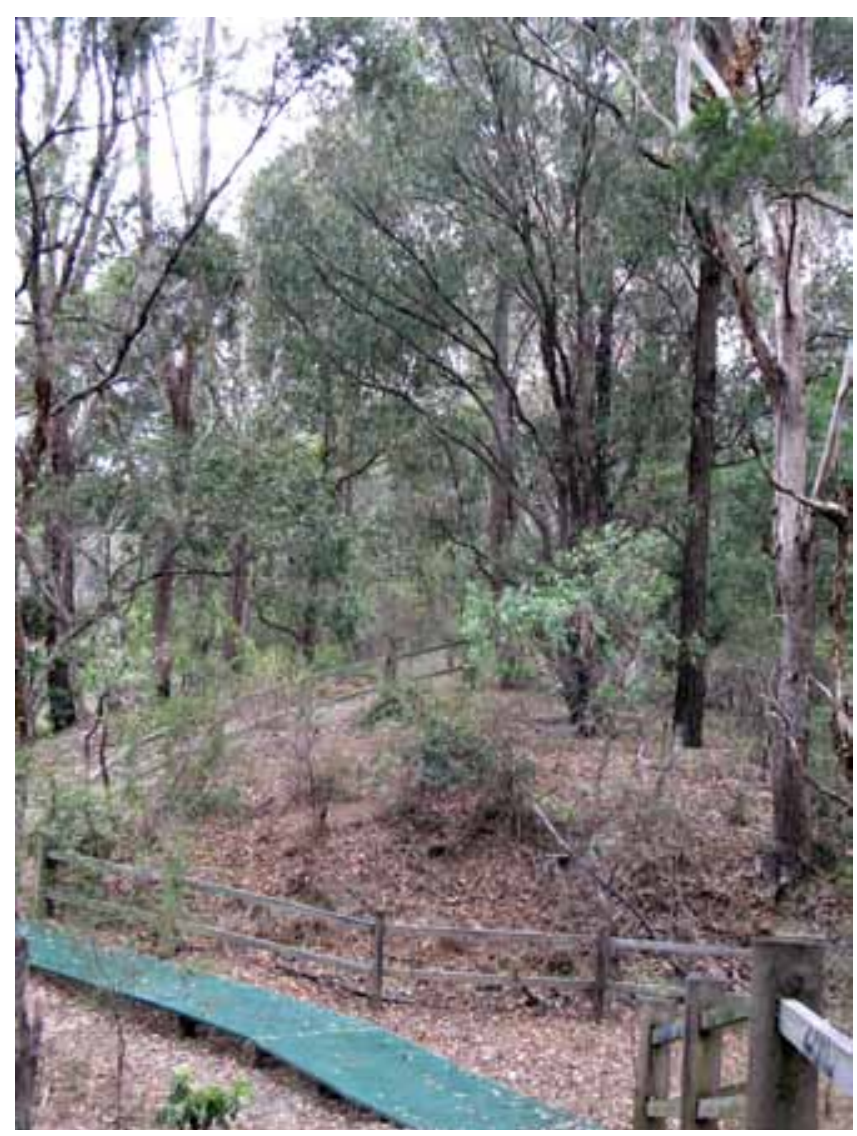

Fig. 8. Parramatta City Council now maintains tracks and fencing at Duck River to minimise erosion and protect the area from trail bike riders as was initially proposed by the Friends of Duck River under Tony Price.

hold different sets of once widespread species. Remnants differ in composition due to fragmentation, differing disturbance histories and chance survivals in some, with species made rare by virtue of their being confined to just a few sites. This is another reason that these small remnants of native vegetation are of great conservation value.

\section{e) Surveys and rare species}

Smith \& Smith (1999) compiled a list of plant species recorded in Rookwood up to 1999 (i.e. their own survey plus Price (1979), Mount King Ecological Survey (1992), Quality Environmental Management (1994) and Teresa James (NPWS (1997)). They listed 608 species for Rookwood, 327 native and 281 exotics, including 115 native and 95 exotic species recorded since 1979 . The additional species may indicate surveys of more stringent sampling design, but may also indicate vegetation change. Certainly the many additional exotic species recorded in the decades since 1979 indicate additional weed encroachments. It is also noted that while Price recorded a large number of grasses present in the cemetery in the late 1970s he did make a note to the effect that 'graminae, poorly collected, Rookwood', and the list of grasses (now Poaceae) has been added to substantially in later years.
Smith \& Smith (1999) noted also that several native species, collected from Rookwood prior to Price's work (known from old herbarium records), were not recorded by him, nor have been since, and are almost certainly no longer there - these include Tetratheca juncea and Bothriochloa biloba lodged in 1913 and 1935, respectively. There are also a small number of species recorded from Rookwood cemetery before and after Price's surveys that do not appear in his lists e.g., Dianella revoluta 1973 \& 1995; Boronia polygalifolia 1912 \& 1997; and Calotis lappulacea 1887 \& 1992 (lodged records viewed via Australia's virtual herbarium website). It may be that these species did not flower and were therefore cryptic and/ or simply overlooked by Price at the time of his surveys. It should be noted that, at the time of Price's work, plant identification was a considerably greater challenge without access to experts and herbarium collections, and that while Beadle, Carolin and Evans' Flora of the Sydney Region (1972) provided a major spur to field botany, there were not the many flora guidebooks or electronic resources that have become available since.

No prior or subsequent flora survey of Duck River has been located that is as comprehensive as that of Price (1979). In herbaria across Australia there exist only a few dozen specimens from Duck River that predate the work of Price, among them material collected by Robert Brown in 1802, Joseph Maiden 1887, A.A. Hamilton 1909, and R. Coveny, D. Benson and H. Bryant 1976 (Australia's virtual herbarium website). And the few surveys since Price (e.g., Greening Australia 1990, NPWS 1997, Applied Ecology 2011), aside from verifying the dominant and common species in the field, all acknowledge that they are largely based on Price's 1979 account.

\section{f) Conservation status of the sites today}

The National Trust recognises Rookwood as a cemetery of World Heritage significance for both its cultural and nature conservation values. It is listed on the Register of the National Estate and under the NSW Heritage Act. While there is enormous pressure on all land within the cemetery for burial space, the vegetation conservation areas are also protected under the Rookwood Necropolis Property Management Plan 2002, which is a statutory document under the NSW Threatened Species Conservation Act, 1995. The Plan of Management recognises the regional significance of the remnant indigenous vegetation, and as a part of the fabric of the cemetery to be preserved and maintained (Rookwood Visual Significance Study 2010). Whilst it has not received the same level of external recognition, the Duck River Reserve in the Parramatta Local Government Area at South Granville is zoned 2EC for conservation, with strong council commitment to impact management and overall protection. 


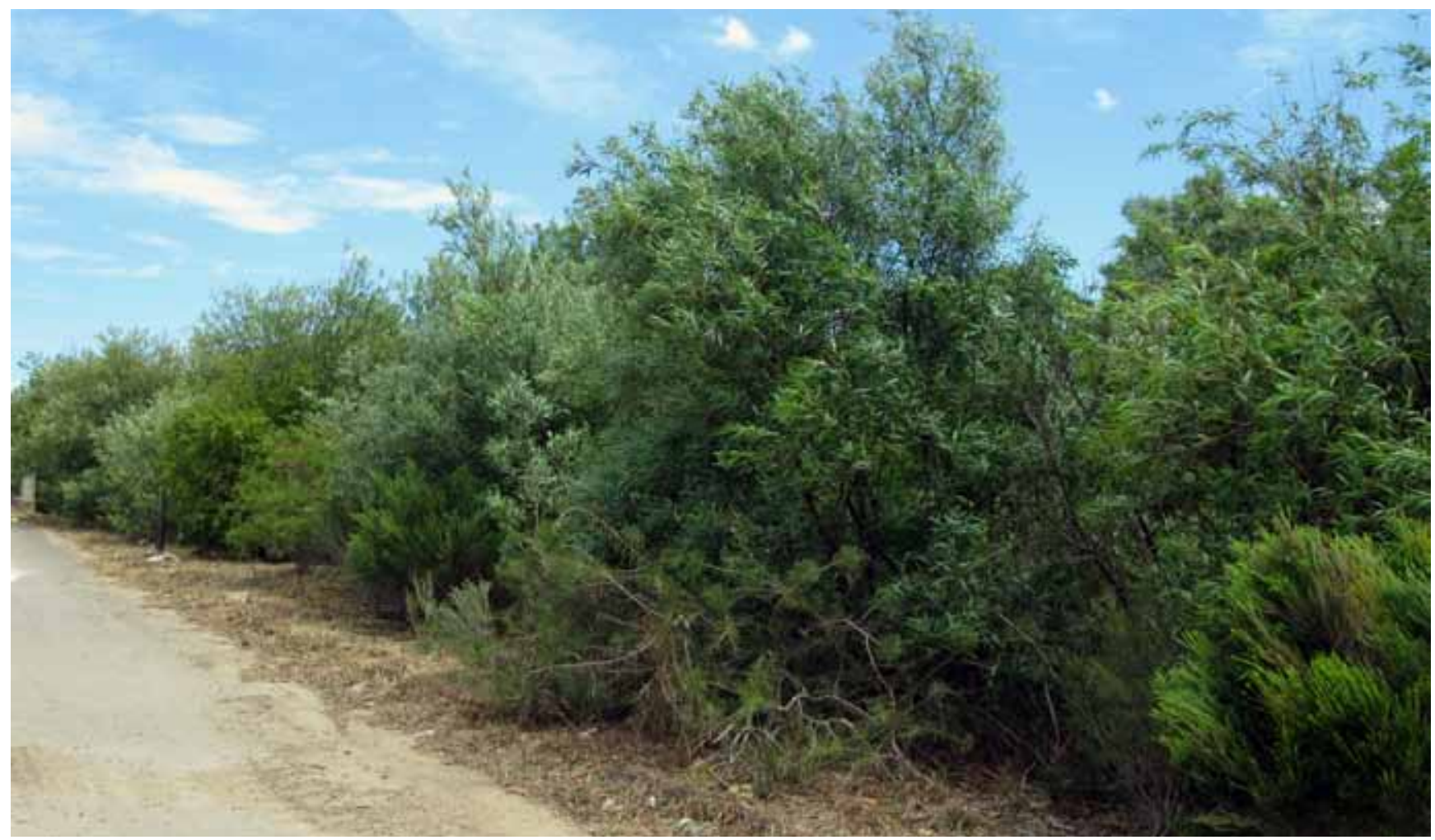

Fig. 9. Rookwood roadside native plantings: A number of perimeter and roadside plantings made within the cemetery since 2005 comprise native species grown from seed collected on site and germinated in the Rookwood Necropolis Joint Committee's nursery located on Hawthorne Avenue. In this planting - Melaleuca thymifolia, Melaleuca erubescens, Dillwynia sieberi, Callistemon linearis, Acacia pubescens, Acacia longifolia.

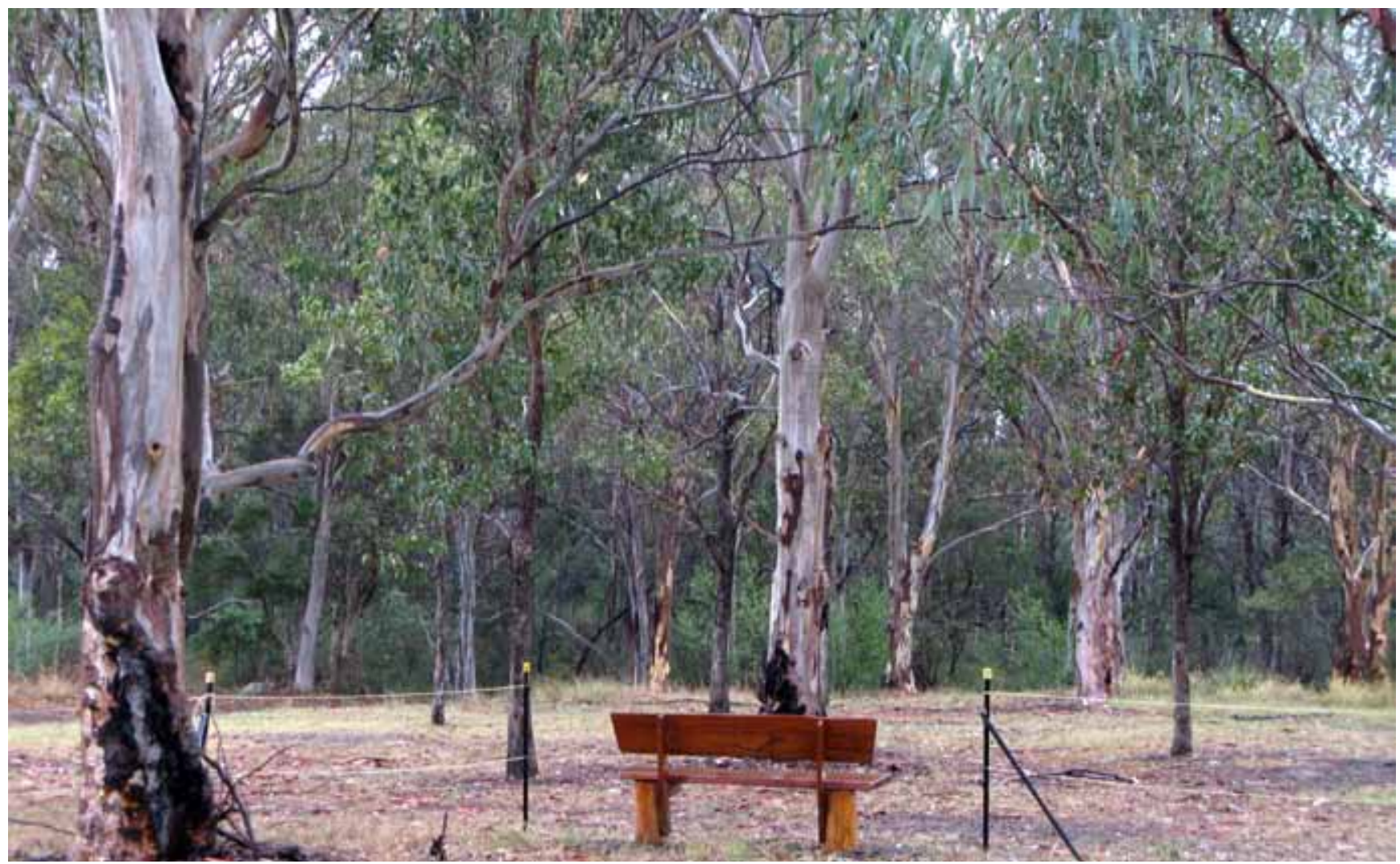

Fig. 10. At the Wellington Road end of the Duck River Reserve a wooden seat dedicated to the memory of Tony Price has been placed looking into the stately Eucalyptus amplifolia. Further into the reserve the track winds past Eucalyptus moluccana, Eucalyptus fibrosa, Eucalyptus longifolia and an unusual stand of Eucalyptus punctata. 


\section{Conclusion}

In the 1970s, at a time when major conservation efforts were being directed at broad scale issues such as the protection of mangroves, rainforests and the development of national park systems, Tony Price's work focussed on the details of remnant vegetation in an increasingly modified suburban landscape. He saw value in this for what it revealed about the past landscape, as well as the ongoing ecological patterns. He concentrated on the careful recording of plant species and observing the ecology of plant responses to environmental conditions such as moisture, shading and fire. As a result his work is a valuable record of the presence, distribution and abundance of plant species at Rookwood and Duck River in the late 1970s, useful for comparing with the present and future times, as well as providing a picture of the district's past as seen from that viewpoint in time.

His observations that rare native species could survive in small areas such as parts of cemeteries, in spite of human impact, highlighted the importance of small areas for conservation, and provided local conservation groups with arguments for improved protection and management of such sites. Similarly, his careful observations of the ecology of native and exotic species set the scene for the development of regeneration and management programs by local conservation groups in subsequent decades.

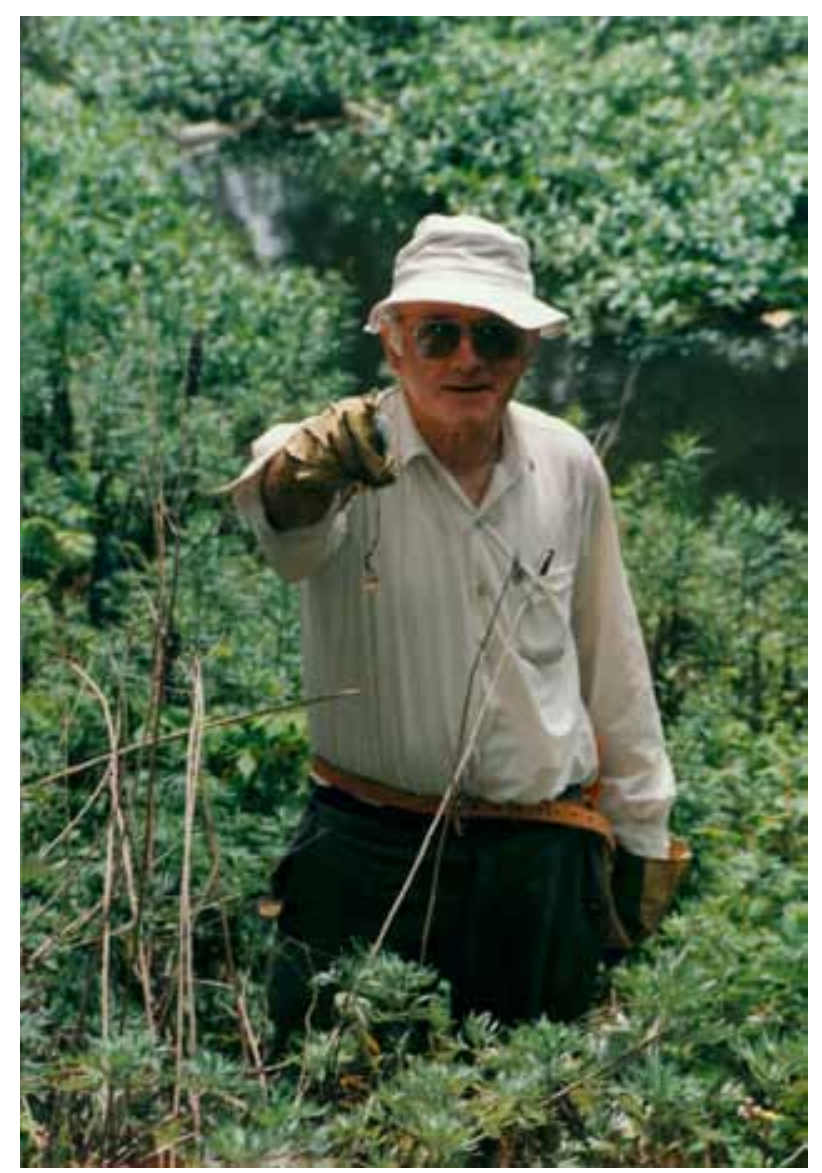

Fig. 11. Tony Price working with other volunteers at Duck River Reserve in January 1998.
Until his death in 2010 Tony Price was still being consulted for his botanical knowledge and ecological opinion which were held in high regard. His work is testament to the value of long term citizen science and community involvement in environmental management. Tony Price joins a long tradition in botany, and the natural sciences more broadly, of citizen contribution and amateur/ professional collaborations (see Gilbert 1982 for interesting examples).

Despite competing landuse priorities at both Duck River and Rookwood, these bushland remnants still survive and certainly remain worthy of protection. As Tony Price was deeply aware, they are important for their inherent conservation values, for the clues they can give us to the past, for the regionally rare and significant flora they hold, for dependent fauna, and as a source of genetic variability for seed banking, horticulture and revegetation projects.

\section{Acknowledgements}

I would like to thank A. Prof. Paul Holford (University of Western Sydney), A. Prof. Michelle Leishman (Macquarie University), Prof. Lesley Hughes (Macquarie University), Doug Benson (Royal Botanic Gardens, Sydney) and Peter Lister (University of Western Sydney) for helpful discussion and constructive comments on earlier drafts of this work. Thank you to my primary supervisor A. Prof. Charles Morris (University of Western Sydney) for his ongoing support and patience. Thanks also to the Friends of Duck River Reserve, staff of Parramatta and Auburn Councils, Mrs Margot Wood in New Zealand and the staff and contractors at Rookwood Necropolis for access to old reports and for sharing their memories of Tony Price.

*This work was supported by a bequest from Greville Anthony (Tony) Price to the Department of Biological Sciences, Macquarie University.

\section{References}

Applied Ecology Pty Limited (2011) Core Bushland Flora and Fauna Surveys Parramatta Local Government Area

Auburn Municipal Council (1982) Liberty Plains: A History of Auburn, N.S.W. (George Lewis Group, Auburn)

Australian National Botanic Gardens, Australian National Herbarium (Jan-May 2012) Australian Plant Name Index APNI http://www.cpbr.gov.au/apni/databases

Australia's Virtual Herbarium (Nov. 2012) http://avh.ala.org.au/ search

Bale, C.L., Williams, J.B. \& Charley, J.L. (1998) The impact of aspect on forest structure and floristics in some Eastern Australian sites. Forest Ecology and Management 110: 363-377.

Barrett, G. W. \& T. L. Barrett (2001) Cemeteries as repositories of natural and cultural diversity. Conservation Biology 15(6): 1820-1824.

Benson, D. \& Howell, J. (1990a) Sydney's vegetation 1788-1988: utilisation, degradation and rehabilitation. Proceedings of the Ecological Society of Australia 16: 115-127.

Benson, D. \& Howell, J. (1990b) Taken for Granted: the Bushland of Sydney and its suburbs. Kangaroo Press: Sydney) 
Benson, D., Ondinea, D. \& Bear, V. (1999) Missing Jigsaw Pieces: The Bushland Plants of the Cooks River Valley (Royal Botanic Gardens: Sydney).

Benson, D. \& Howell, J. (2002) 'Cumberland Plain Woodland ecology then and now: interpretations and implication from the work of Robert Brown and others'. Cunninghamia 7(4): 631-650.

Bond, W.J., Woodward, F.I. and Midgley, G.F. (2005) The global distribution of ecosystems in a world without fire. New Phytologist 165: 525- 538.

Branagan, D. F., Herbert, C. \& Langford-Smith, T. (1979) An outline of the geology and geomorphology of the Sydney Basin. Marrickville, N.S.W., Science Press for the Dept. of Geology and Geophysics, University of Sydney.

Burke, B. \& Betteridge, C. (1989) From bush to burial ground: the landscape and design of a garden cemetery. In: The Sleeping City. The Story of Rookwood Necropolis. (D. A. Weston, Society of Australian Genealogists in conjunction with Hale and Iremonger), 61-74.

Chapman, G. A. \& Murphy, C.L. (1989) Soil Landscapes of the Sydney 1: 100000 Sheet. (Soil Conservation Service of NSW, Sydney)

DEM Australia Pty Limited (2006) Rookwood Necropolis Property Management Plan Audit, Section 2.1, (DEM Chatswood, NSW 2067).

DEM Australia Pty Limited (2010) Rookwood Visual Significance Study, (DEM Chatswood, NSW 2067)

EDAW Australia Pty Limited (1996) Duck River Management Plan. (Duck River Steering Committee).

EDAW Australia Pty Limited (1996) Appendix C. Fauna List for Duck River.

French, K., Callaghan, B. \& Hill, S. (2000) Classifying endangered vegetation communities: a case study of Cumberland Plain Woodland. Pacific Conservation Biology 6 (2): 120-129.

Gilbert, L.A. (1982) Plants and Parsons in Nineteenth Century New South Wales. Historical Records of Australian Science 5 (3): 17-32.

Greening Australia Limited (1990) The Duck River Open-Space Bushland Survey South Granville, New South Wales.

Granville Historical Society (1992) Granville from Forest to Factory. (David J. File Printers, Seven Hills)

Hill, S.J., Tung, P.J. \& Leishman, M.R. (2005) Relationships between anthropogenic disturbance, soil properties and plant invasion in endangered Cumberland Plain Woodland, Australia. Austral Ecology 30: 775-788.

James, T., McDougall, L. \& Benson, D. (1999) Rare Bushland Plants of Western Sydney (Royal Botanic Gardens Sydney).

Kartzoff, M. (1969) Nature and a city: the native vegetation of the Sydney area (Edwards and Shaw Pty Limited).

Kerr, J. Semple (1985) Cemeteries: Their Value and Conservation. In: Australia in Trust. A Selection of the Best Writing from 'Heritage Australia' Sydney (Australian Council of National Trusts in association with William Collins Pty Limited).

Kohen, J. (1993) Ch. 3 Hunting and Collecting. In: The Darug and their neighbours. (Darug Link in association with Blacktown and District Historical Society), p. 23-30.

Loneragan, W. A. (1975) The ecology of a graveyard. Australian Journal of Botany 26: 803 - 814.

McBarron, E. J., Benson, D.H. \& Doherty, M.D. (1987) The botany of old cemeteries. Cunninghamia 2(1): 97-104.

National Parks and Wildlife Service (1997) Urban Bushland Biodiversity Survey Stage 1: western Sydney (NSW National Parks and Wildlife Service: Hurstville).

Pidgeon, I.M. (1941) The ecology of the central coastal area of New South Wales. IV. Forest Types on soils from Hawkesbury Sandstone and Wianamatta Shale. Proceedings of the Linnean Society of New South Wales 66, 113-137.
Price, G. A. (1979) The Vegetation of Duck River and Rookwood Cemetery, Auburn. (Unpub. Copy attached as Appendix 2)

Price, G. A. (1993) A Conservation and Management Strategy for the remnants of Native Vegetation in Rookwood Cemetery. Appendix 9. In: Rookwood Necropolis Plan of Management 1993.

Rookwood Necropolis Joint Committee (1993) Ch. 3 Conservation Analysis. In: Rookwood Cemetery Plan of Management 1993.

Sirgist, J. (1989) A Walk Through History In: The sleeping city. the story of Rookwood Necropolis. (D. A. Weston, Society of Australian Genealogists in conjunction with Hale and Iremonger), 13-29.

Smith, P. \& J. Smith (1999) Flora and Fauna Survey of Rookwood Necropolis. Report to Joint Committee of Necropolis Trustees.

The Royal Botanic Gardens and Domain Trust (Jan-May 2012). PlantNET - The Plant Information Network System of The Royal Botanic Gardens and Domain Trust, Sydney, Australia http://plantnet.rbgsyd.nsw.gov.au

Tozer, M. (2003) The native vegetation of the Cumberland Plain, western Sydney: systematic classification and field identification of communities. Cunninghamia 8(1): 1-75.

Tozer, M.G., Turner, K., Keith, D.A., Tindall, D., Pennay, C., Simpson, C., Mackenzie, B., Beukers, P. \& Cox, S. (2010) Native vegetation of southeast NSW: a revised classification and map for the coast and eastern tablelands. Cunninghamia 11 (3): 359-406.

Urban Bushland Management (UBM) Ecological Consultants (2011) Rookwood Necropolis Bush Regeneration Annual Report for July 2010 - June 2011.

Watson, P. J., Bradstock, R. A. and Morris, E. C. (2009), Fire frequency influences composition and structure of the shrub layer in an Australian subcoastal temperate grassy woodland. Austral Ecology 34: 218-232.

Manuscript accepted 26 February 2013 


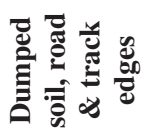

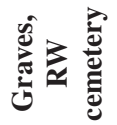

$\mathscr{1}$

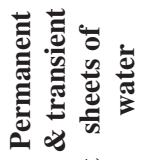

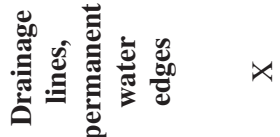

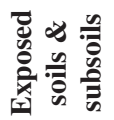

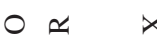

$\circ$

踣

\&

娄

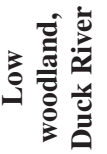

0

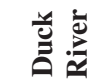

$\times$

○

$00 \simeq 0 x \simeq x \propto 0 \times 0 \times x$

Uัँ

$\simeq \simeq \simeq 0$

$000 \simeq \simeq$

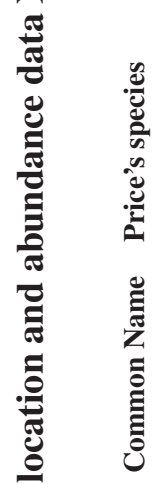
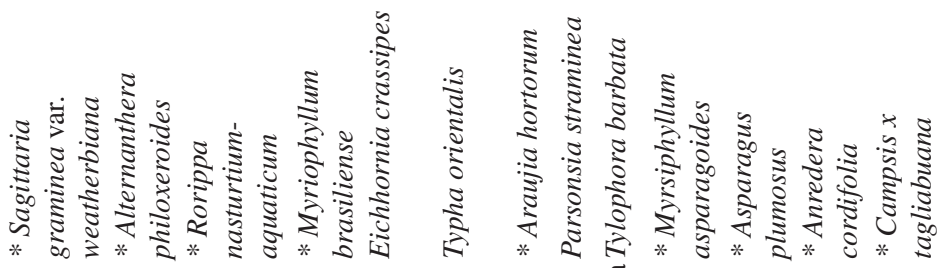

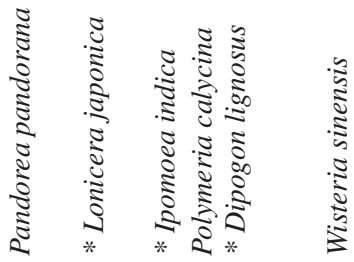

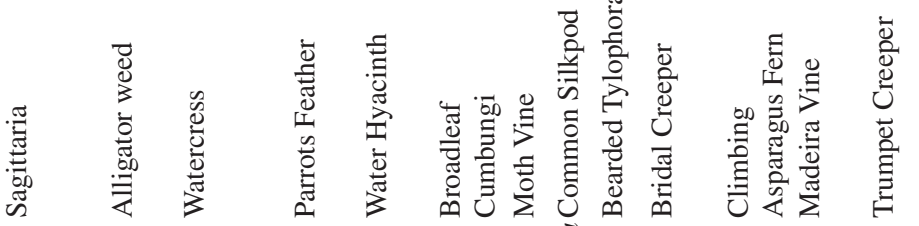

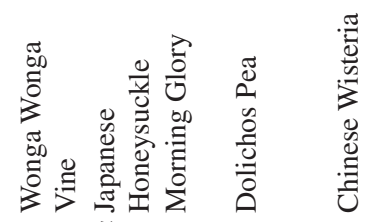

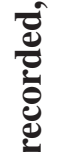

๕

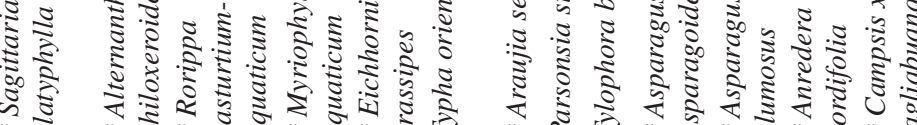

穿离 


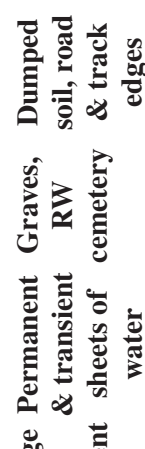

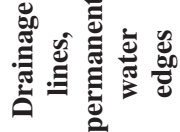

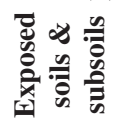

है

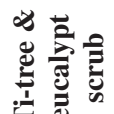

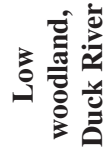

弟苛

这

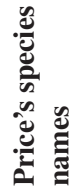

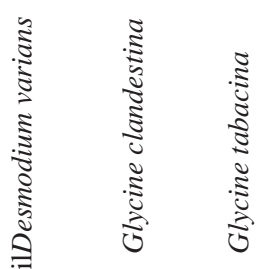

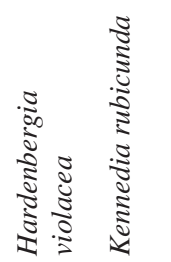
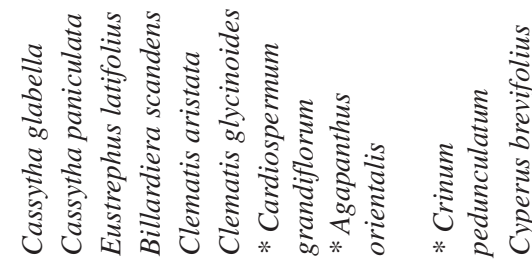

$000 x$

己ัँ

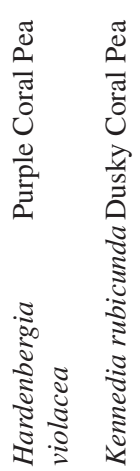

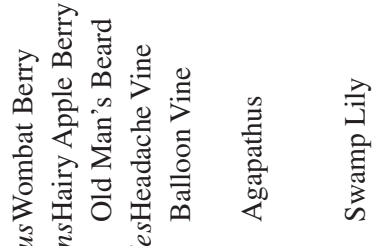

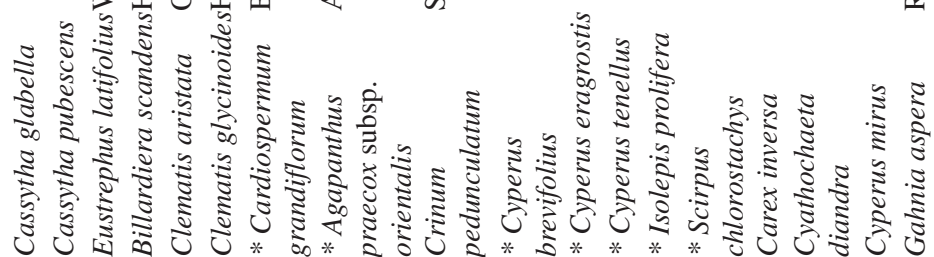

Еัّ

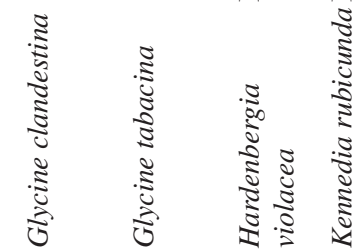

$0 \propto x$

00

$0 x$

竞

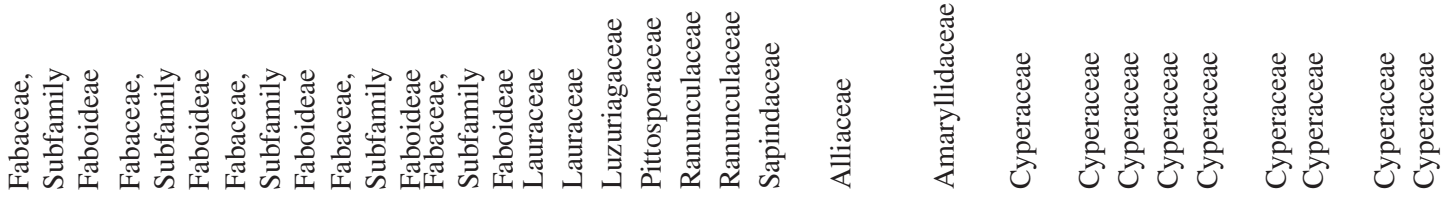

旁

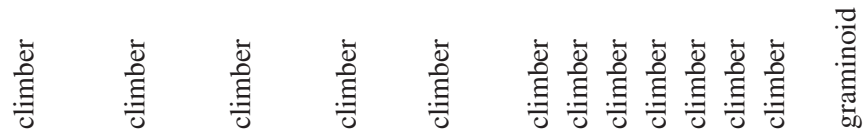




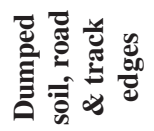

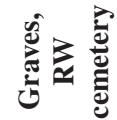

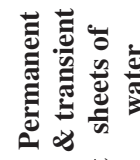

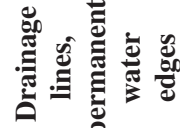

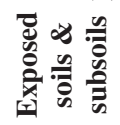

量

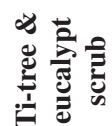

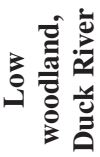

喜离

递

00

o

$0000 \simeq \simeq$

$\simeq x$

$\simeq$

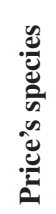

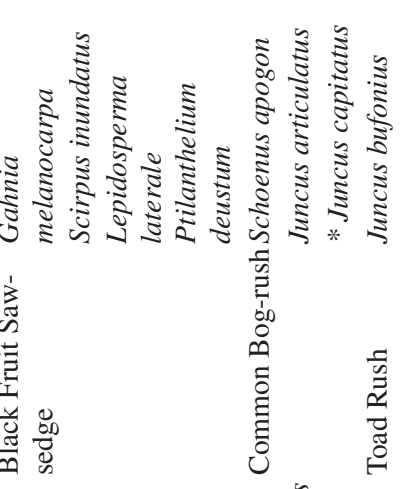

$\simeq \simeq \simeq \simeq \simeq x$

$\circ 0 \times 0$

$x 0 \quad x \approx$

蒙

产

竎竎

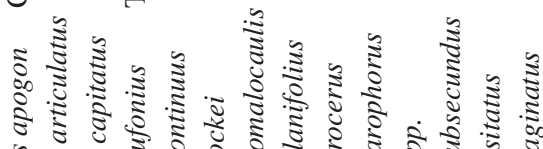

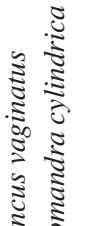

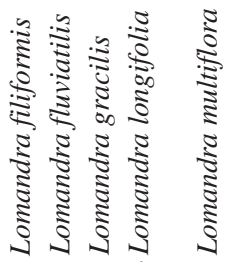

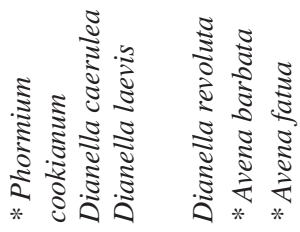

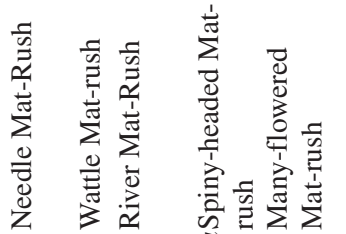

胥

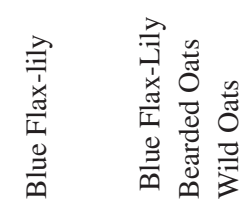

שัّ

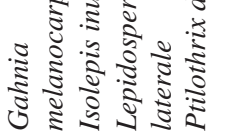

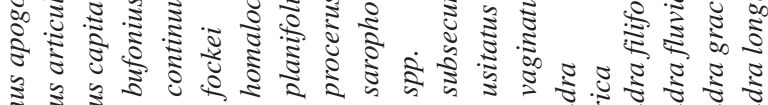

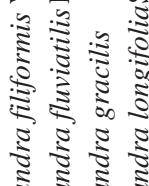

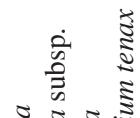

$8: \approx$ : 2

竞

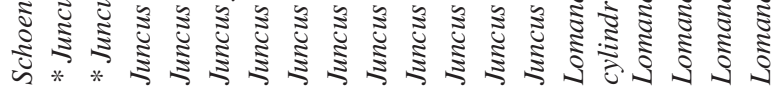

골ำ

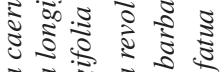

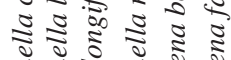

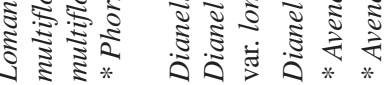

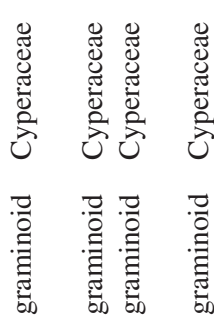

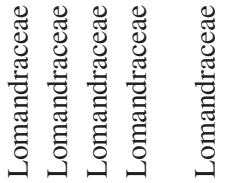

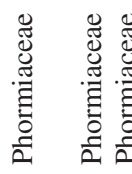

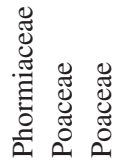

产

WIIID

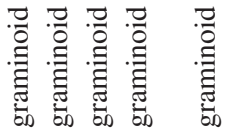

I

翌翌瓷 
MIn $\ldots$

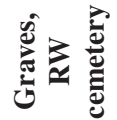

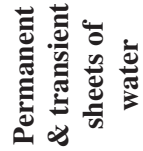

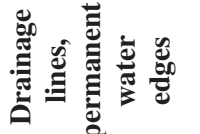

$\simeq 0 \times x \propto \propto ⿻$

$\simeq \simeq \circ \circ \times 0$

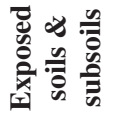

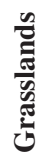

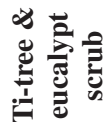

$0 x \simeq 0$

-

ren

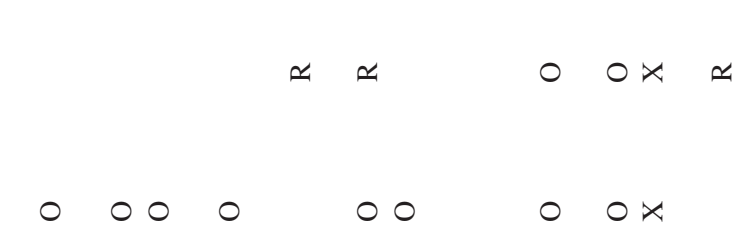

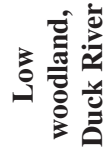

$00 \approx$

○

$\simeq$

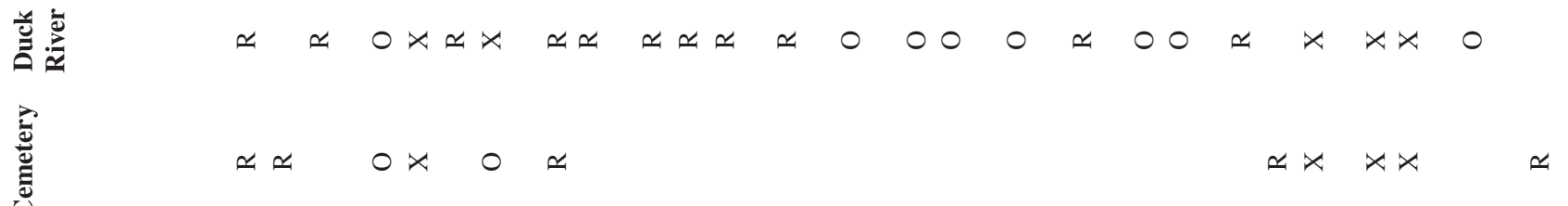

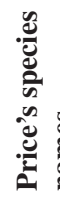

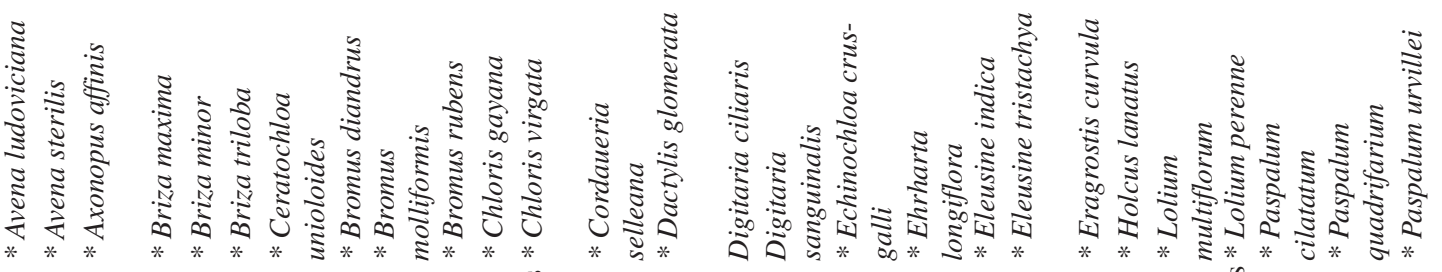

(2)

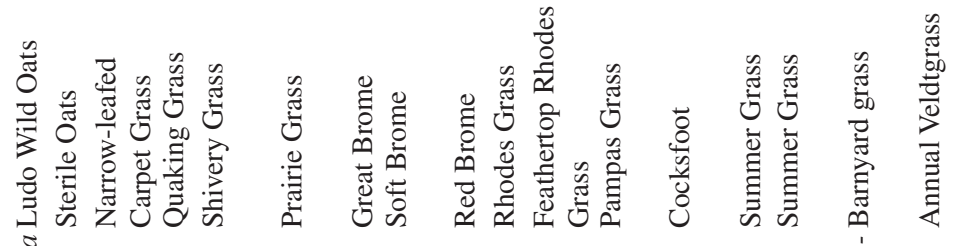

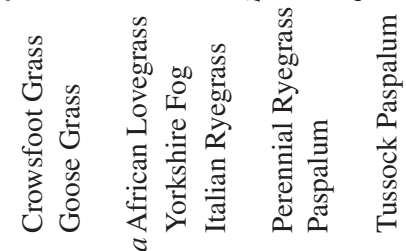

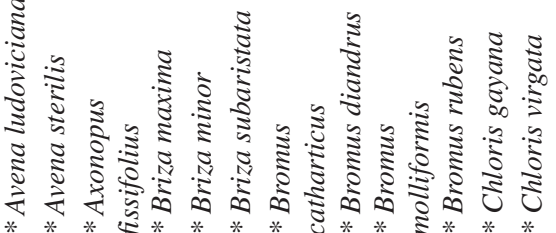

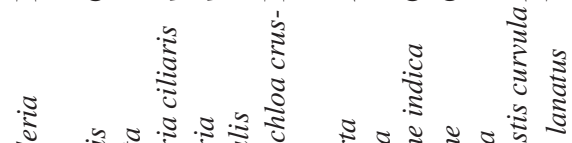

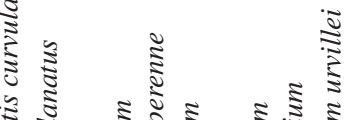

שัّ

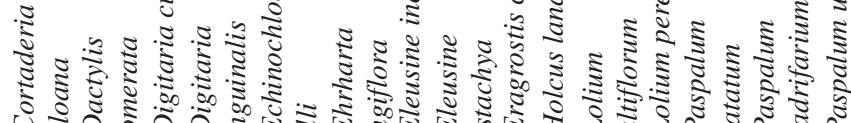

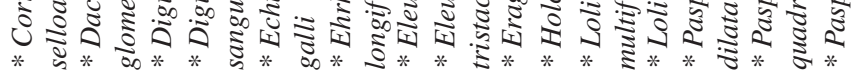

吾

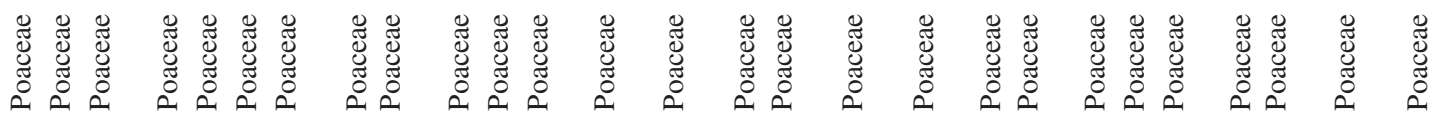

㝇

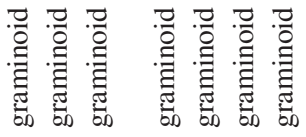

翌

翌量量品

最

臀

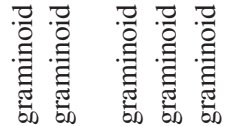

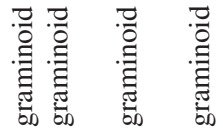




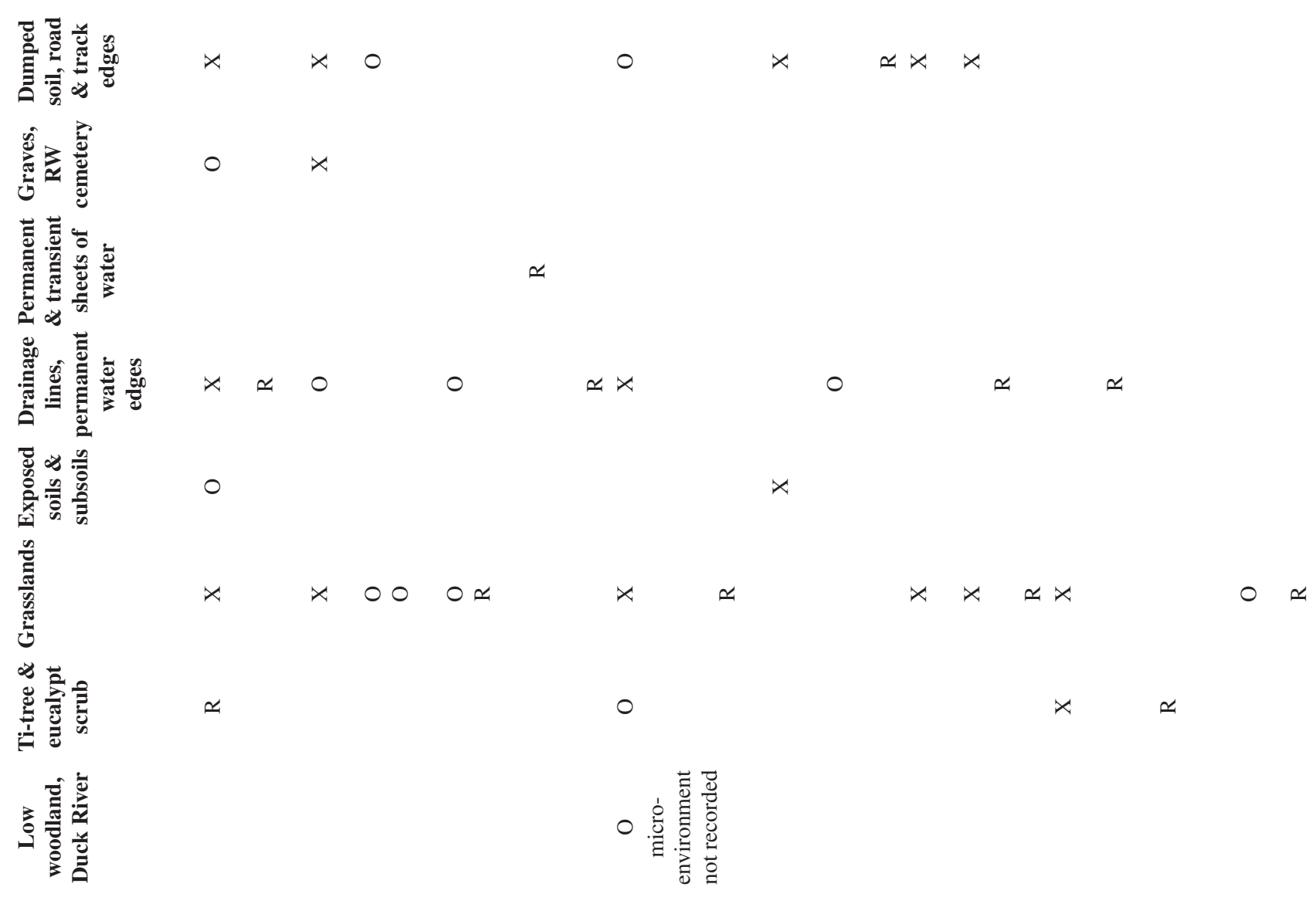

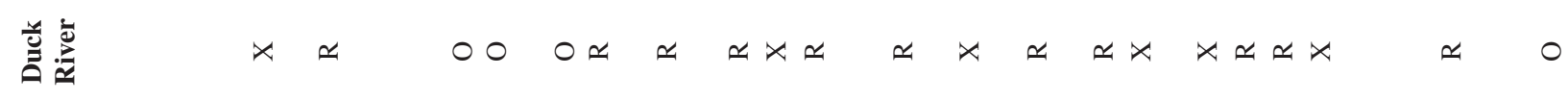

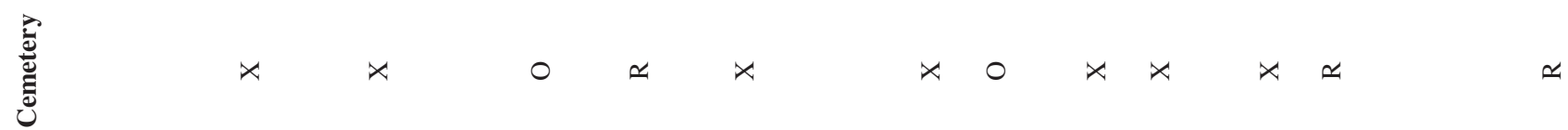

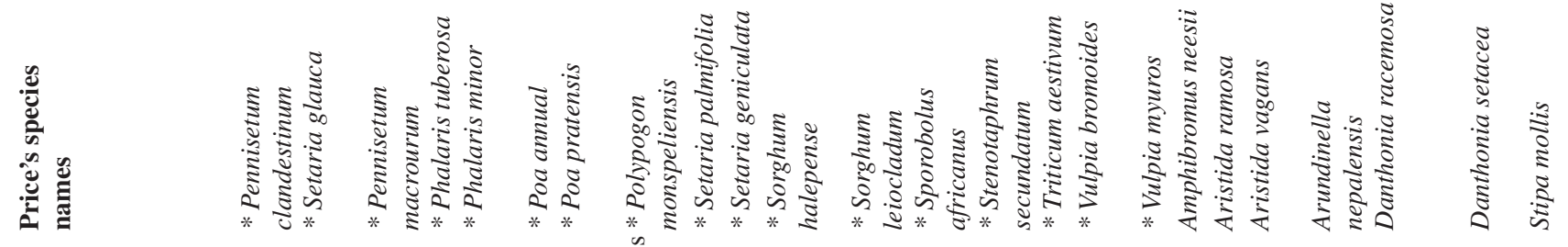

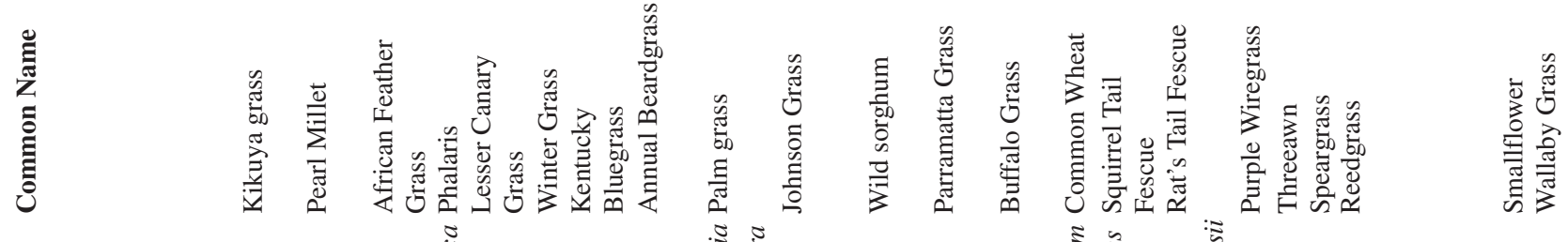

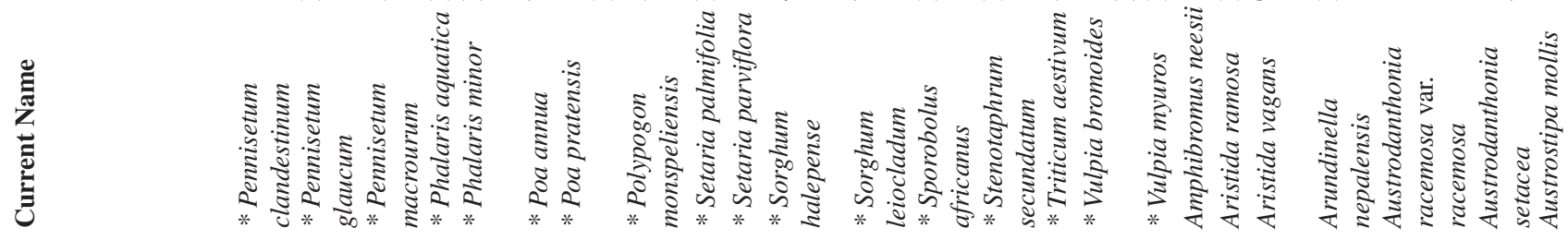

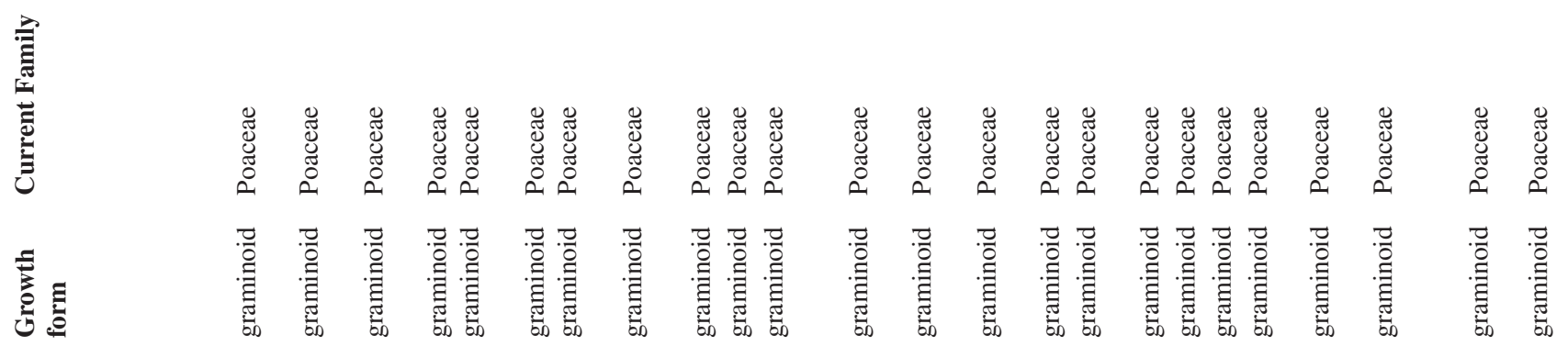




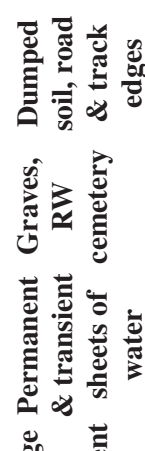

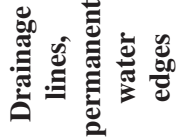

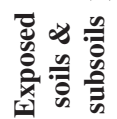

焉

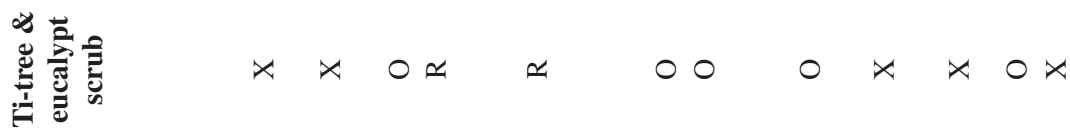

至

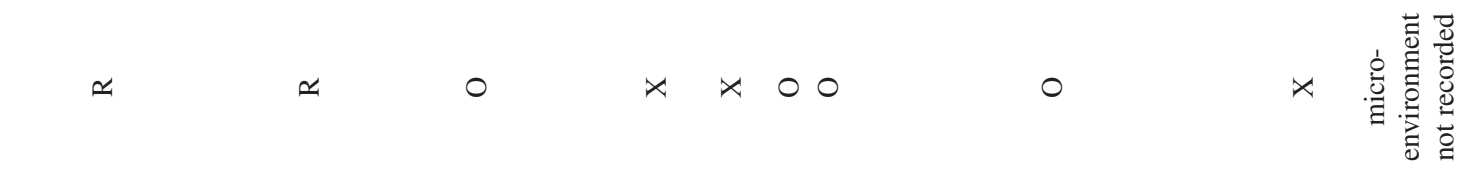

善离

$\times x$

$0 \simeq x$

这

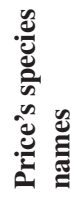

$x \times$

苋

ठี

Uัँ

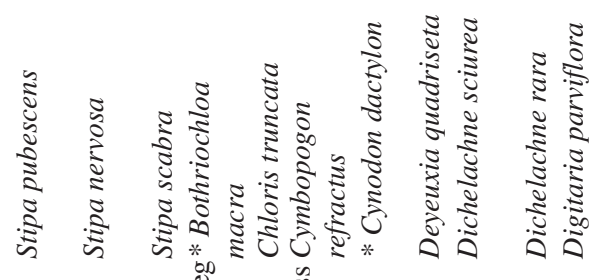

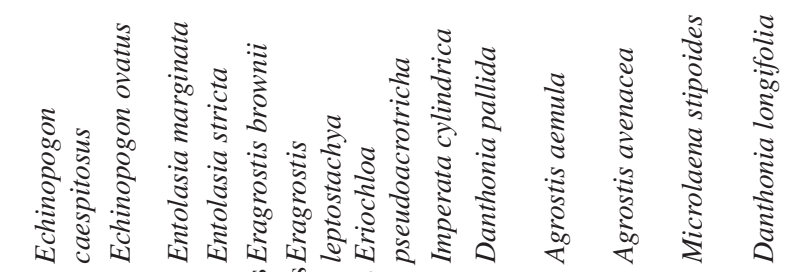

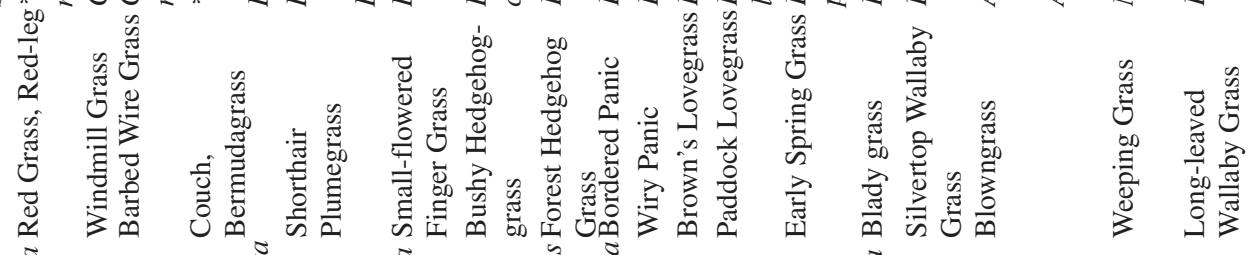

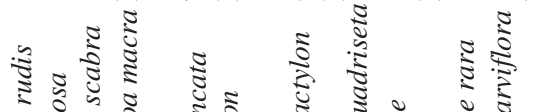

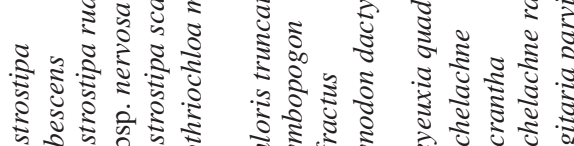
MUM

$\times 0 \simeq$
$\times 0 \simeq$
$x \quad x$

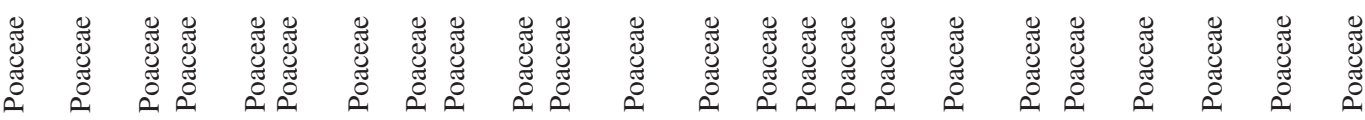

言竞

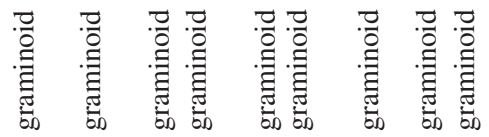

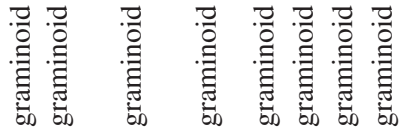

nen

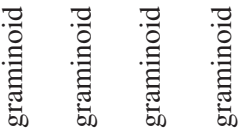




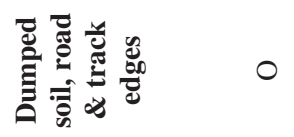

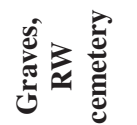

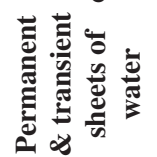

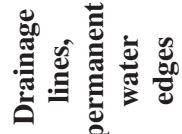

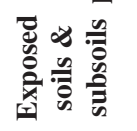

造

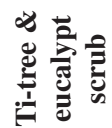

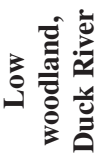

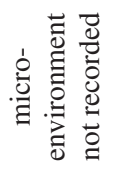

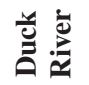

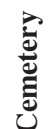

:

己.

嶽

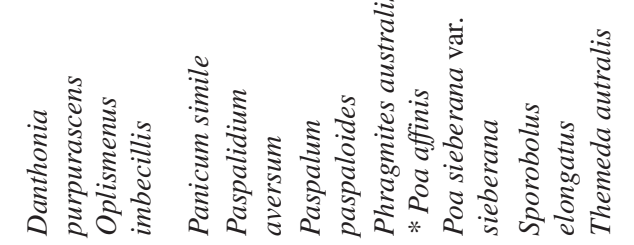

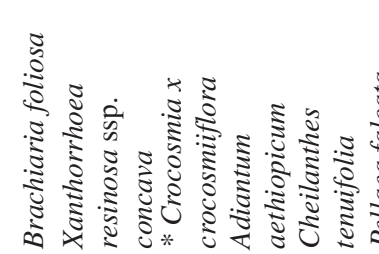

$\frac{\sqrt{3}}{\sqrt{3}}$

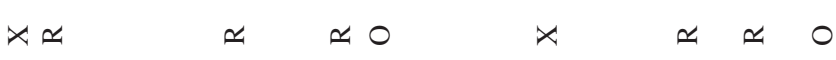

$\simeq 0 \quad 0 x$

$\simeq$

$\alpha$

$\simeq x$

$\times 00$

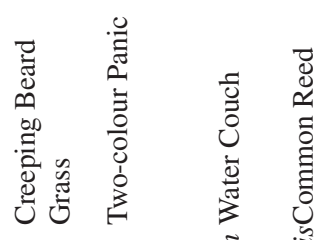

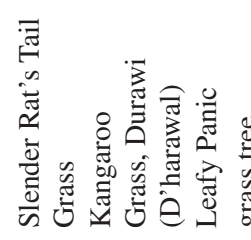

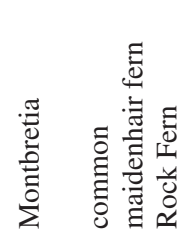

器旁

In

总。

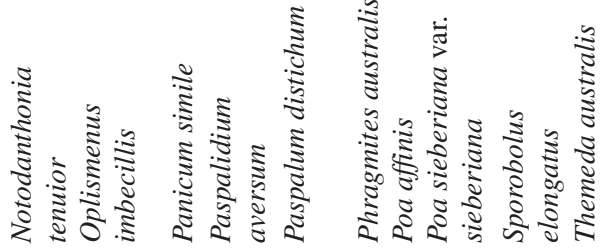

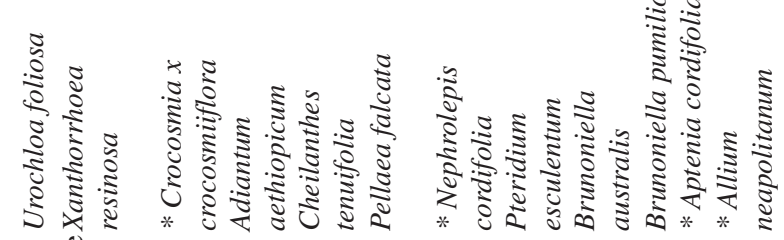

竞

MIIII

11

11111111

II

In

III

1

II

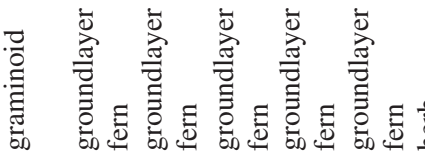

空告

产会亭 8 


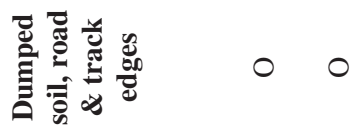

部范密

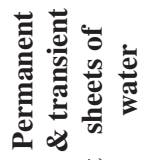

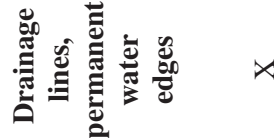

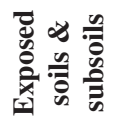

焉

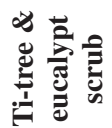

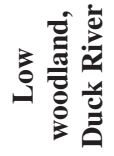

产产

Uัँ

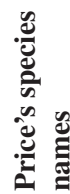

$+1$

压

ठี

$0 \simeq \simeq$

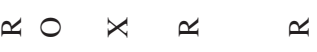

$\simeq$

$\times 000 \quad 0$

$\propto$

$\simeq \times 0 \times x \times x 0$

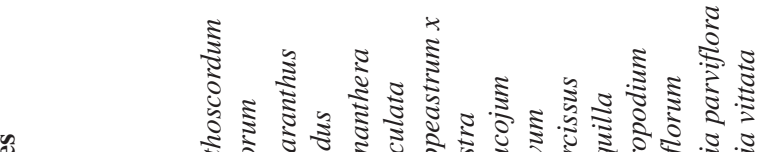

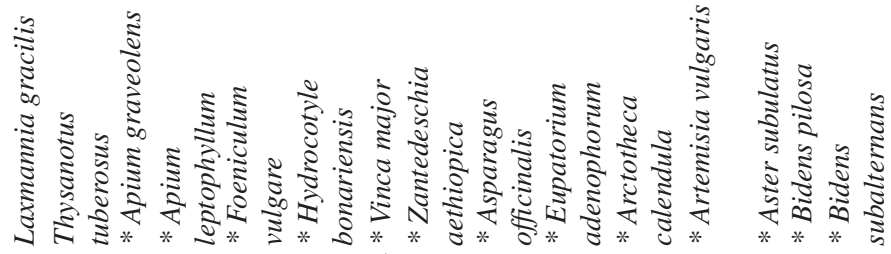

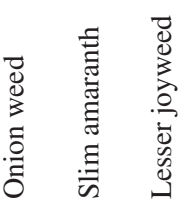

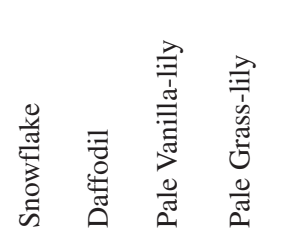

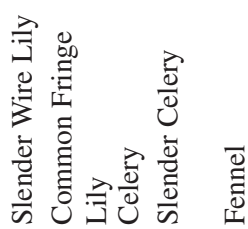

竞

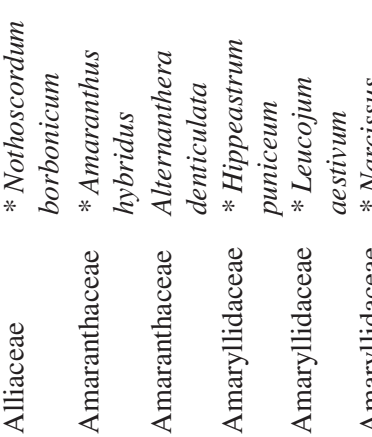

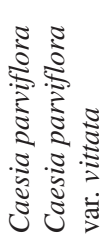

气

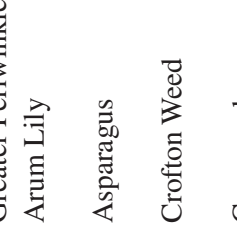

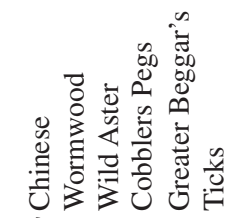

שัّ

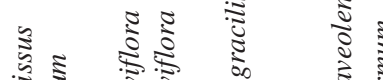

竞

ㄴำ

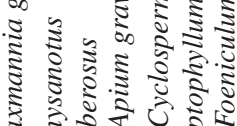

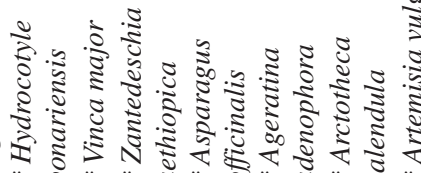

5

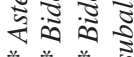

递

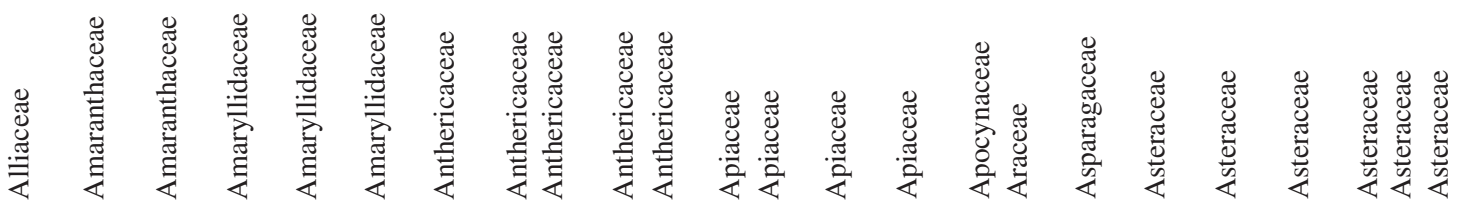

善

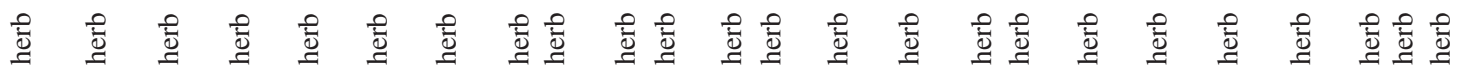




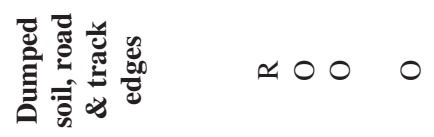

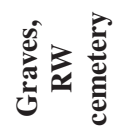

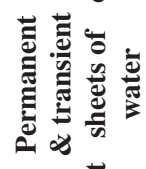

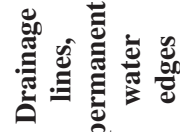

离为语

○

$\simeq$

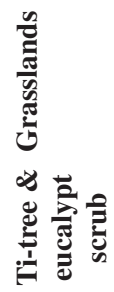

总

$\circ \circ$

$\circ 00$

$x$

$\circ \times$

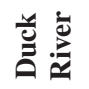

$\simeq 000$

$\circ \circ$

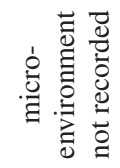

:

$000 \times 0 x$

$\frac{\mathscr{e}}{\tilde{E}}$

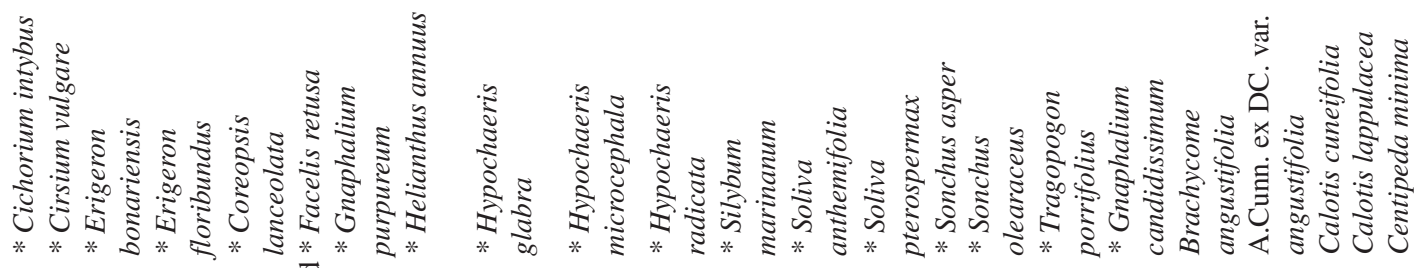

己ั.

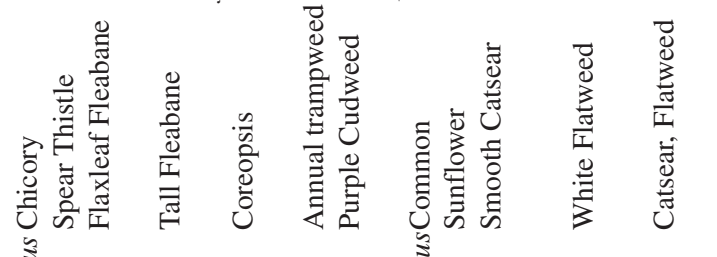

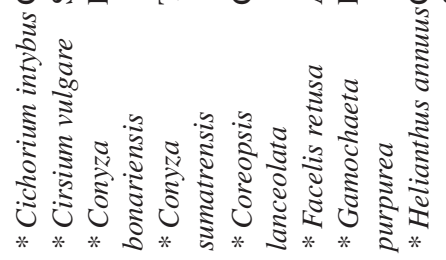

芯

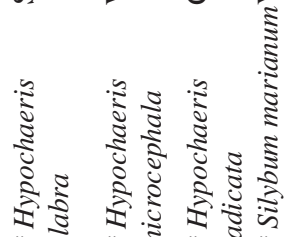

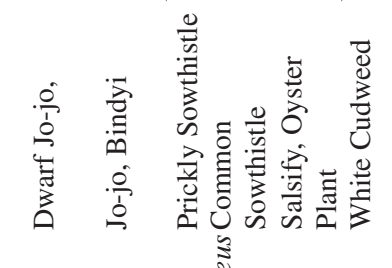

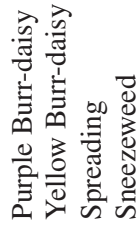

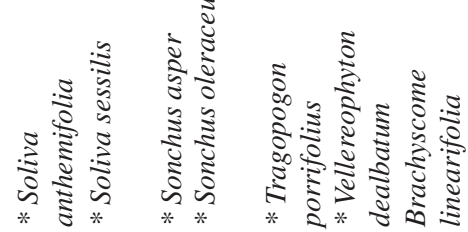

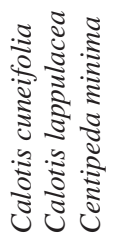

葛

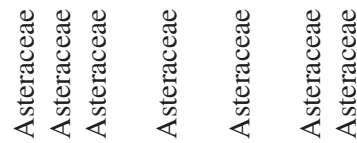

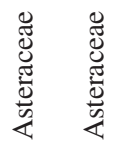

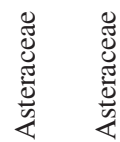

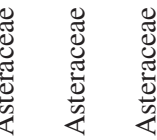

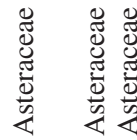

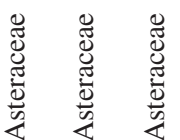

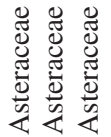

苞

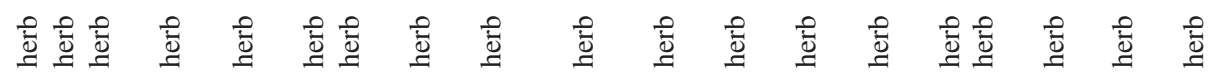

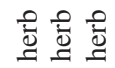




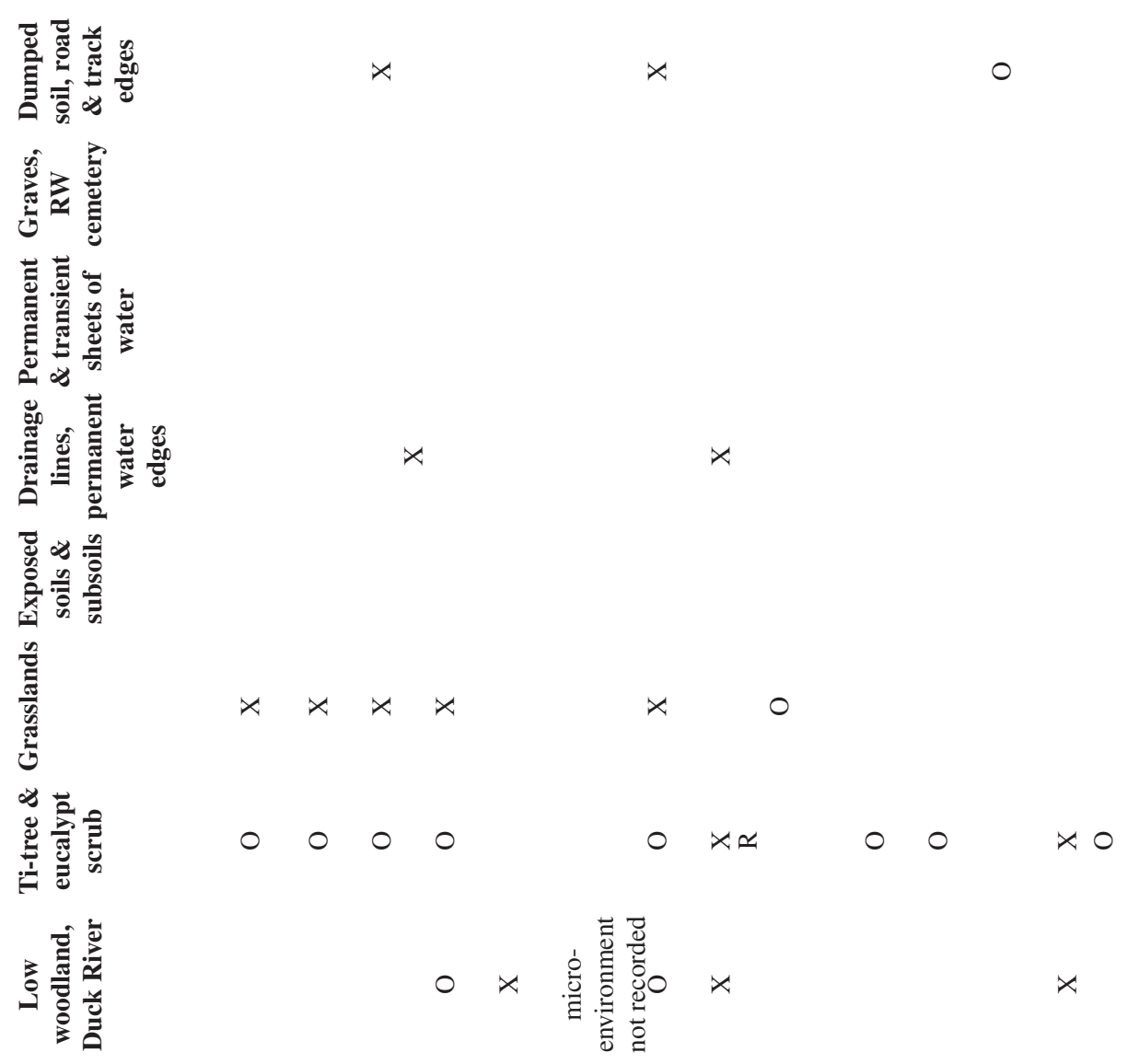

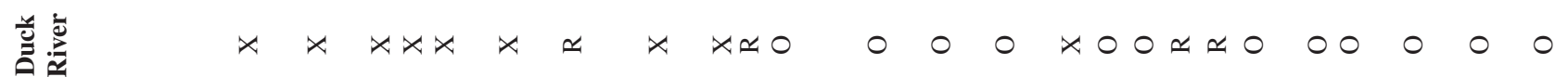

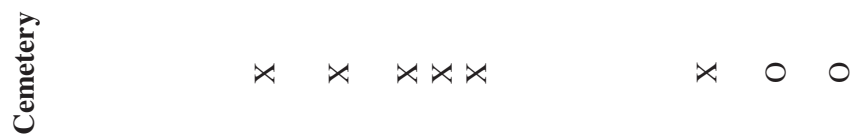
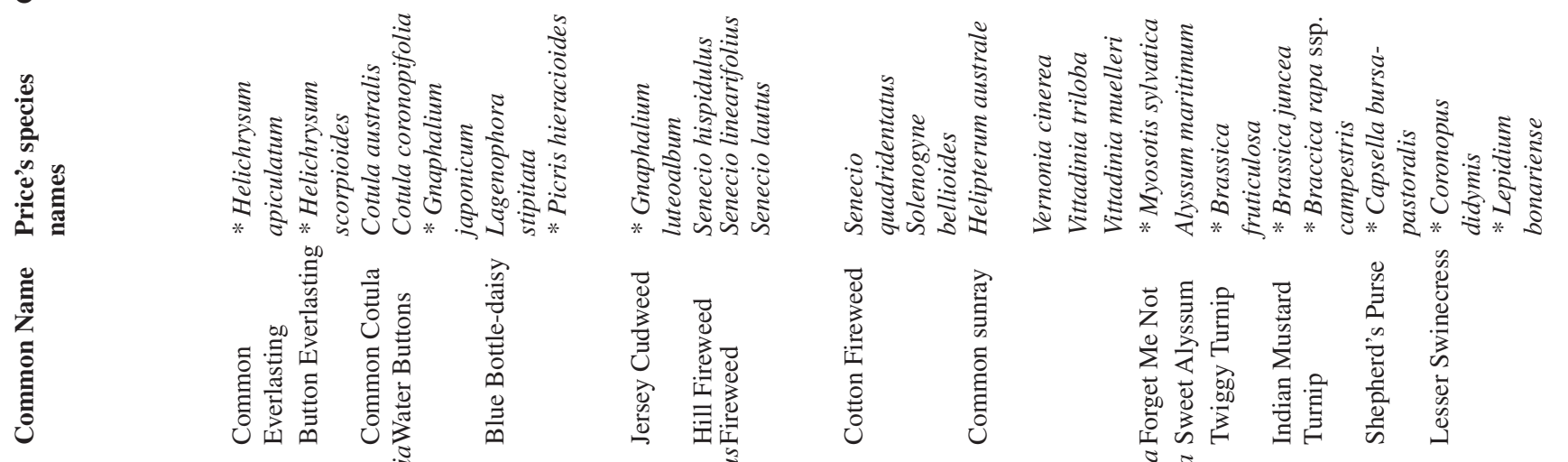

हัँّ

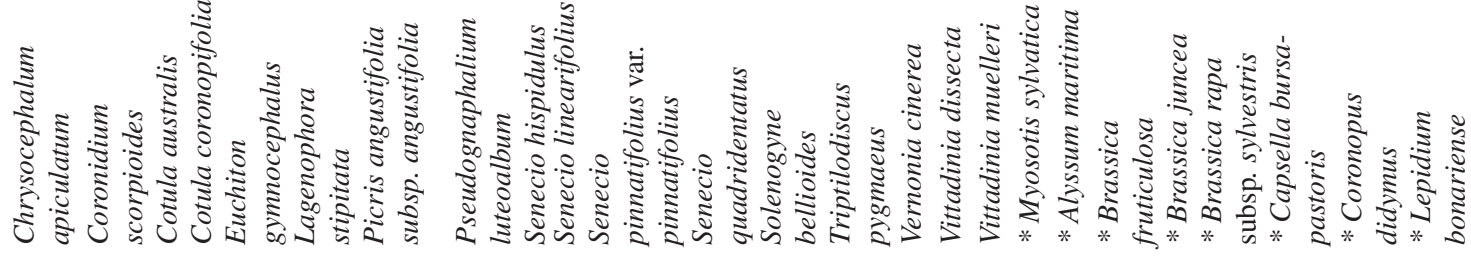

曾
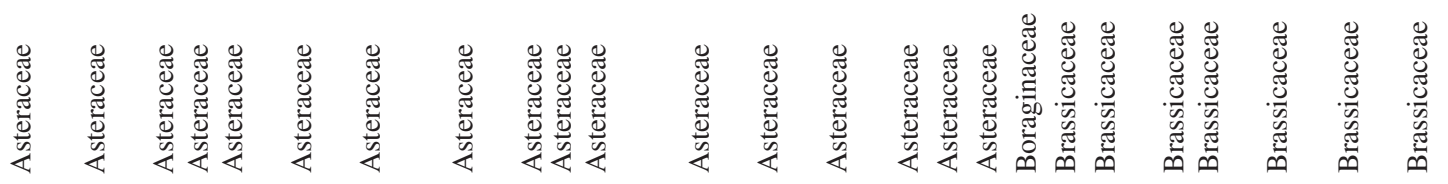

婄的

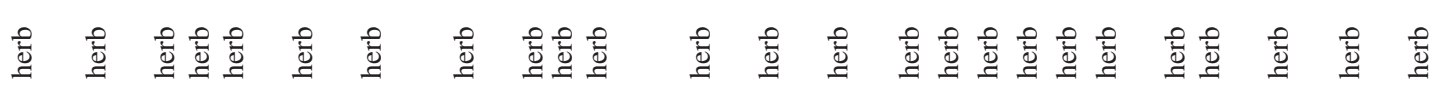




\section{琶影}

$\sum_{0}^{\circ}$

竞苛范

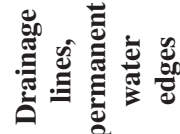

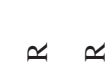

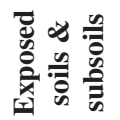

卷

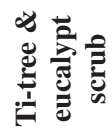

ق气

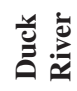

$\simeq \simeq 0 \times x \simeq \simeq 0$

:

$\circ$

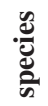

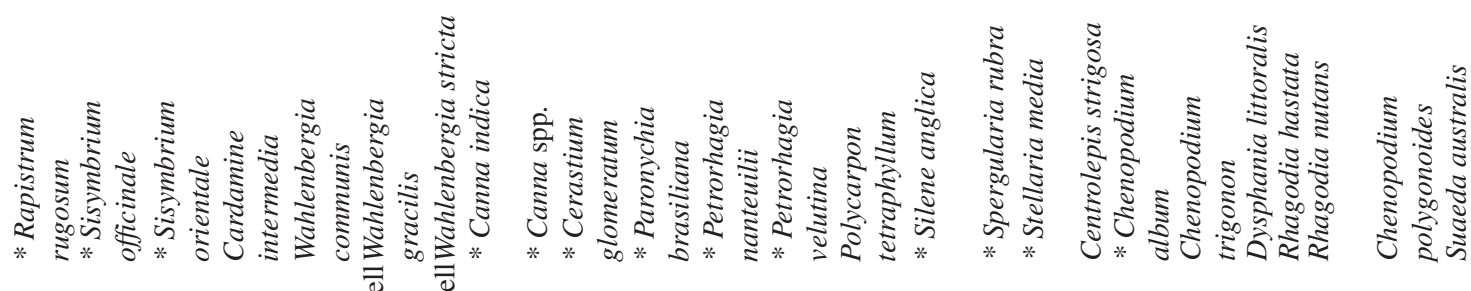

ठั.

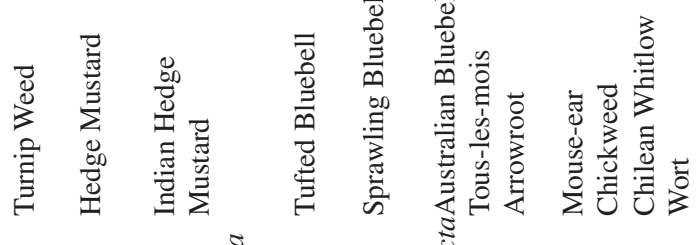

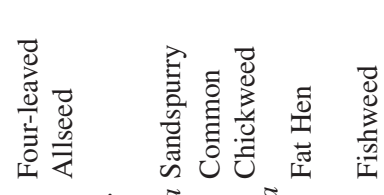

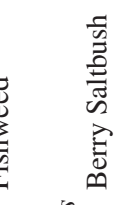

总

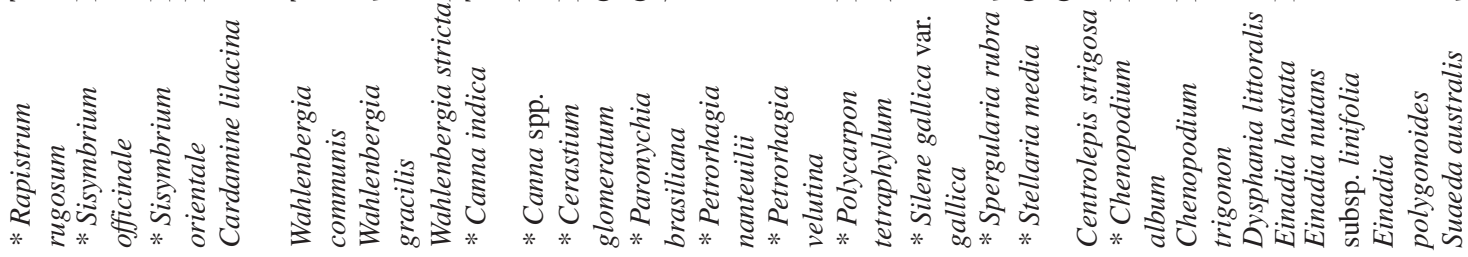

总

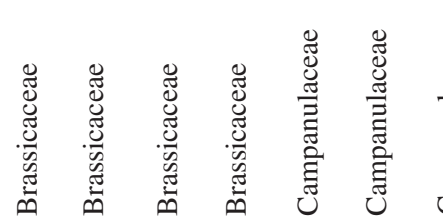

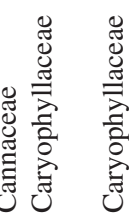

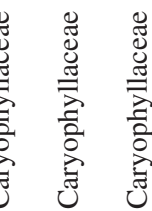

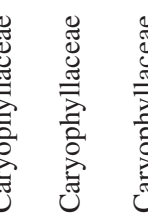

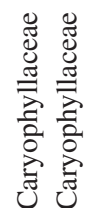

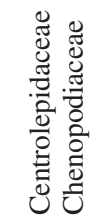

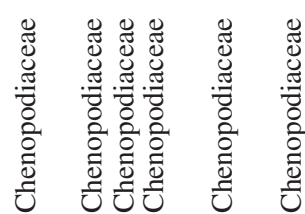

言竞

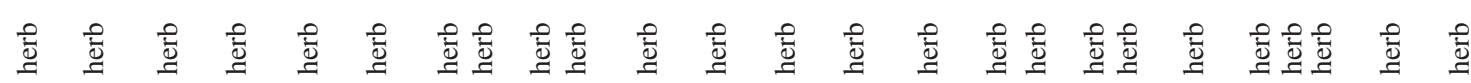




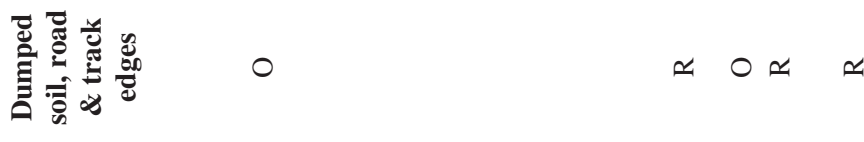

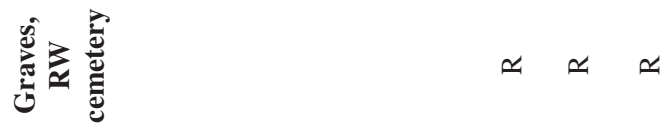

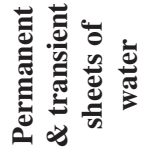

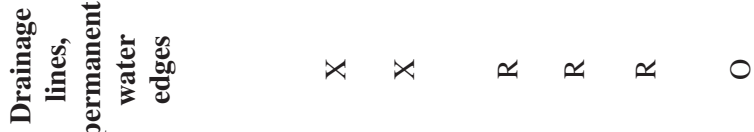$$
\text { 要事 }
$$

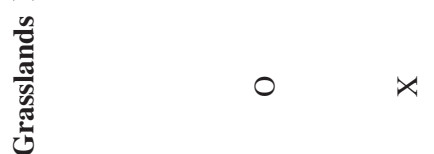

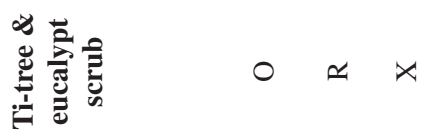

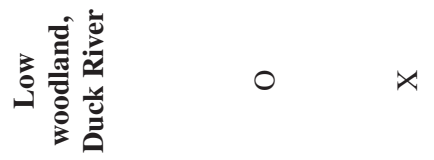

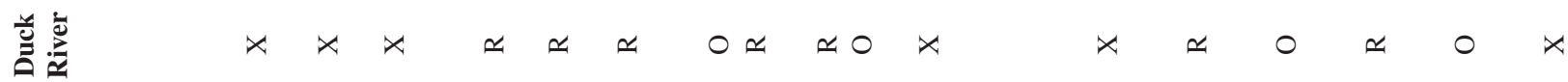

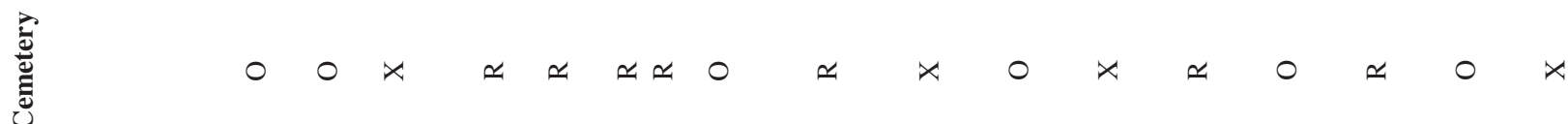
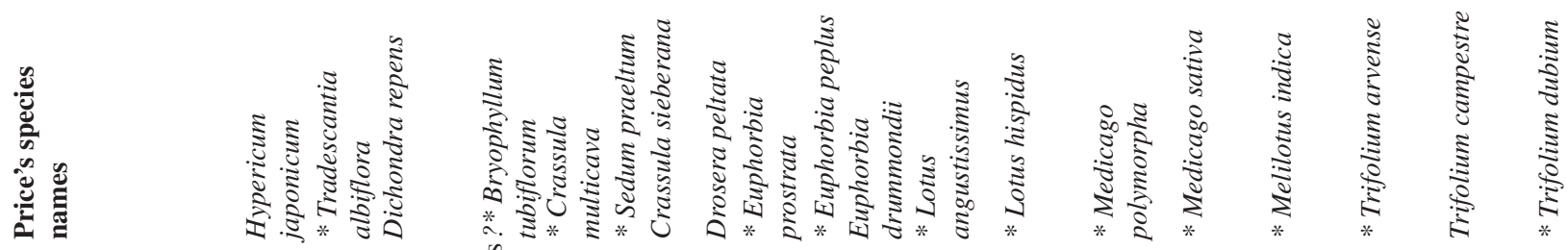

हัँ

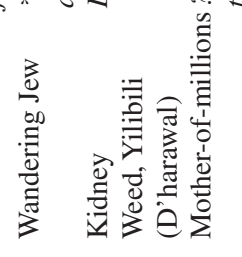

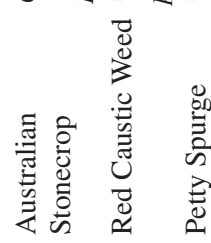

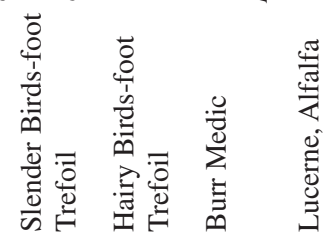

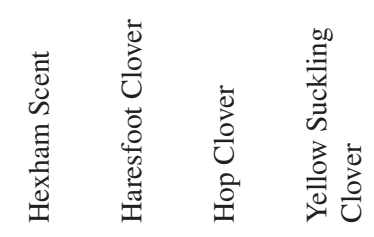

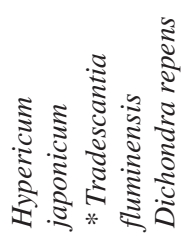
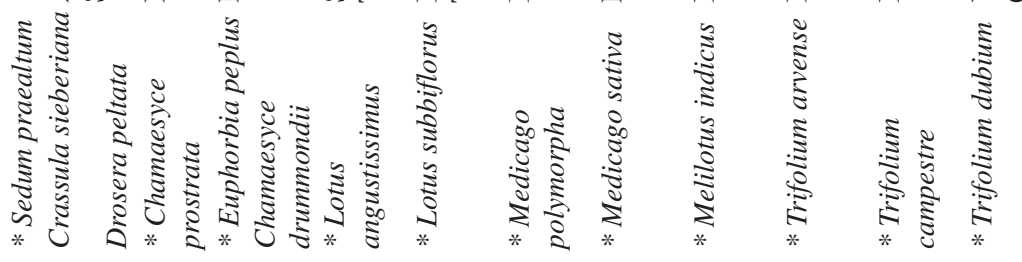

离
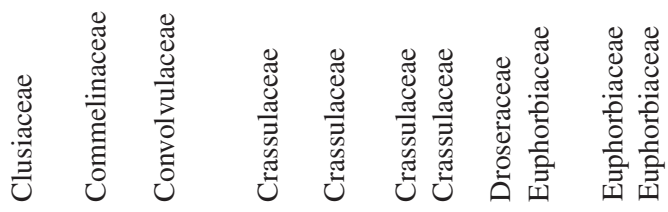

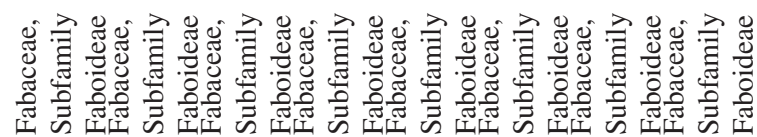




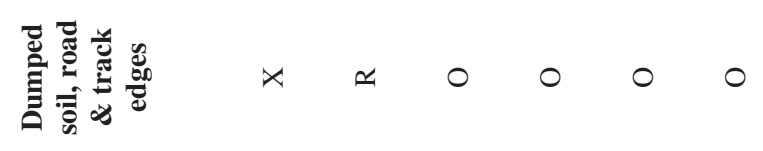

就!

Wis

III!

In:

畒部

㨱

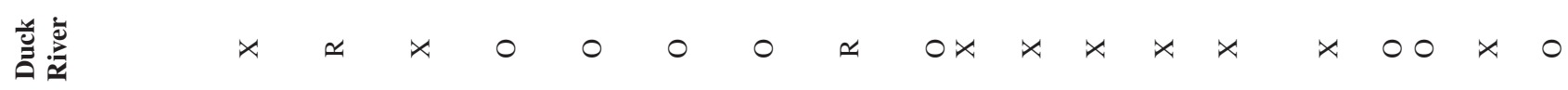

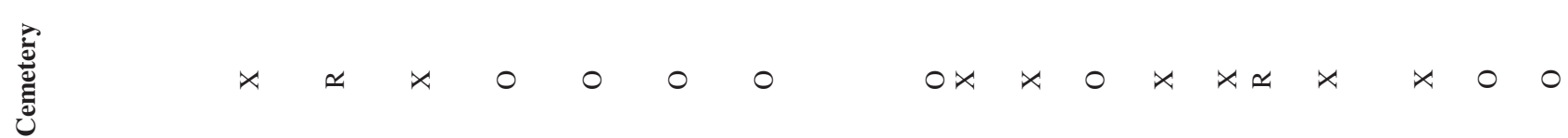

恼

\|\|\|\|

WHWWWU!

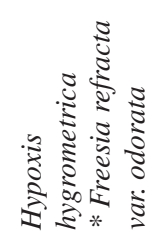

I

高咳

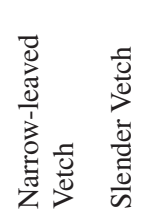

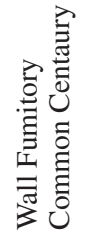

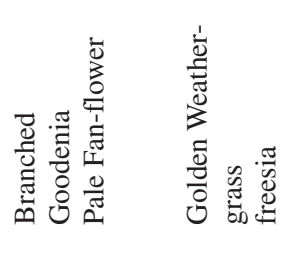

艺

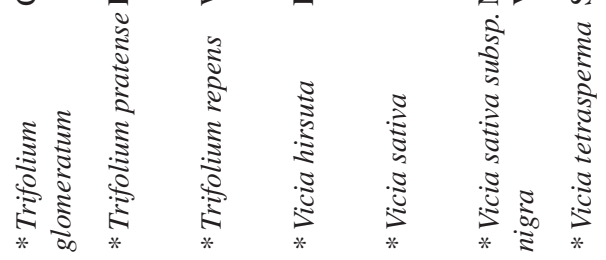

हิ

意咅高

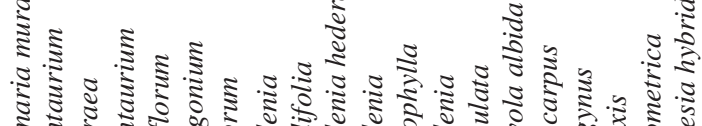

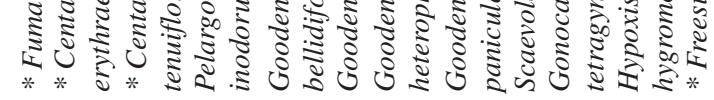

I

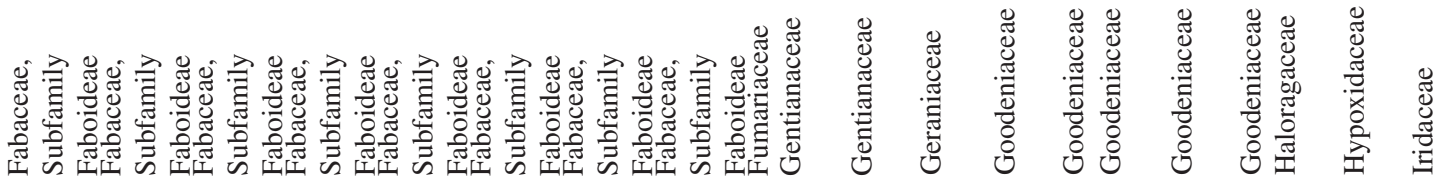

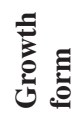

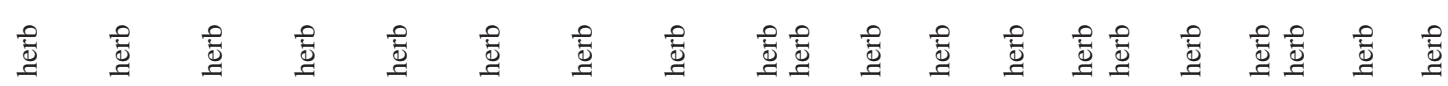


竖部野

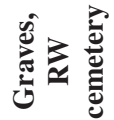

00

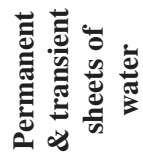

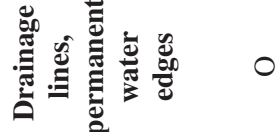

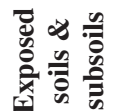

ט.

$\circ \circ \circ \times$

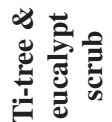

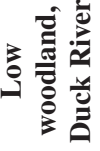

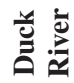

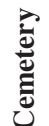

$0 \propto x<\propto x$

$0 x x x \simeq 0 x x 0 \quad 0 \quad 0 \quad x$

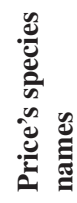

$\circ 0$

$000 \times 0$

$x \propto x$

$x x \simeq$

$0 x \quad 0 x 0 \quad 0 \quad 0 x$

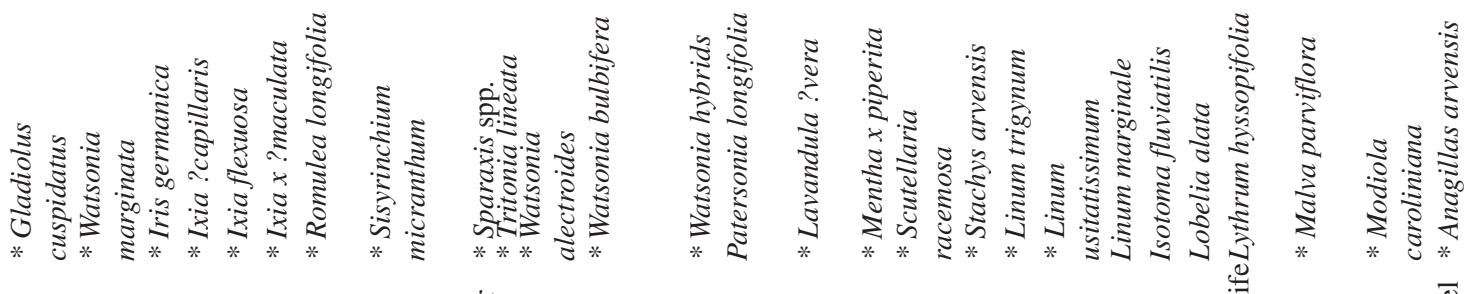

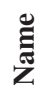

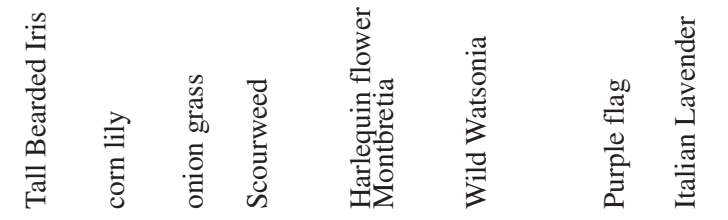

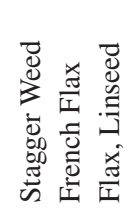

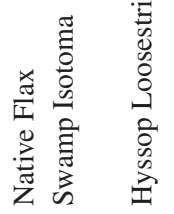

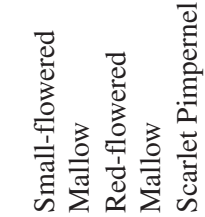

己ัّ

๕

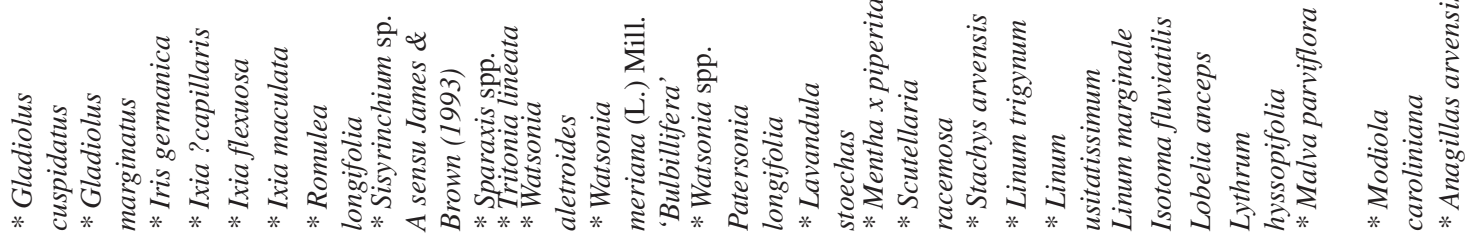

党

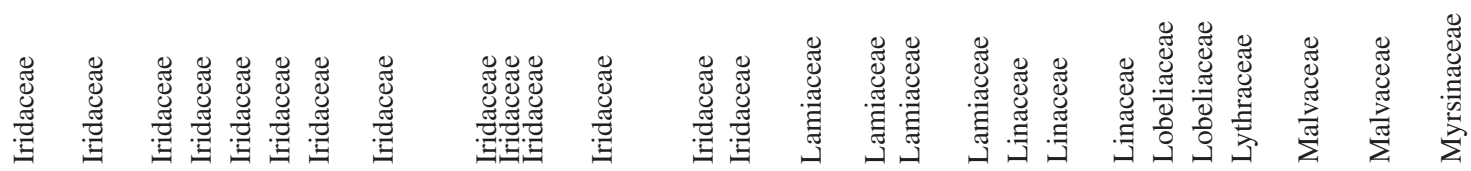

僖

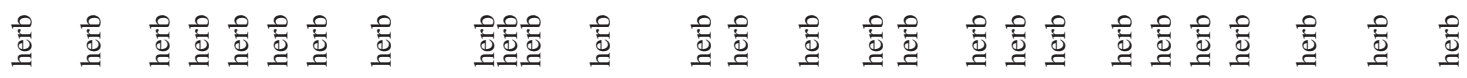




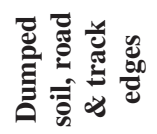

预芯总

Win

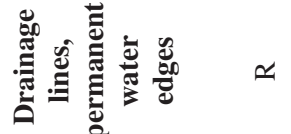

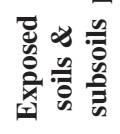

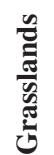

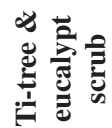

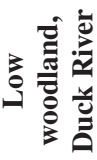

U⿺辶ّ

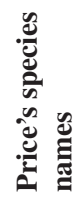

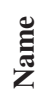

蒫

爮

曾

高咅

$\frac{1}{2} \simeq$

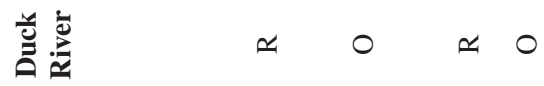

$$
\propto \propto ⿻ \simeq \propto ⿻ 上
$$

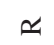

$\simeq \propto$

$x 0 \propto ⿻ x \times x \propto x \simeq x \simeq \simeq$

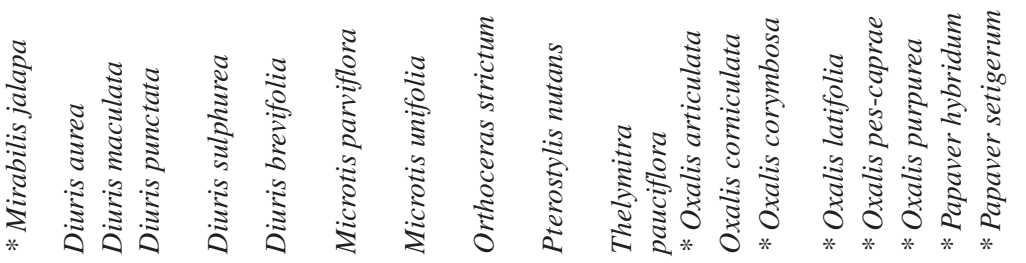

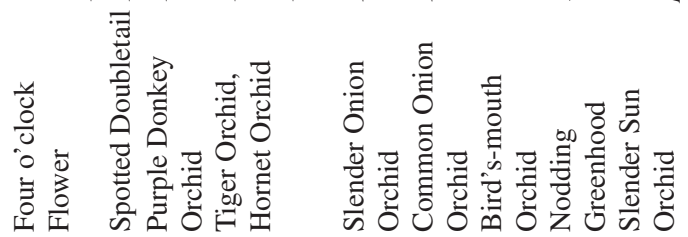

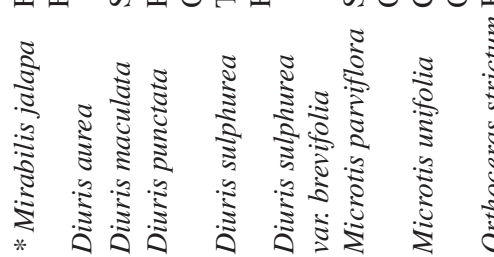

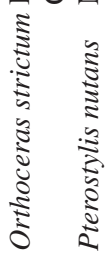

产离

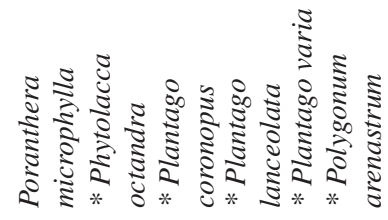

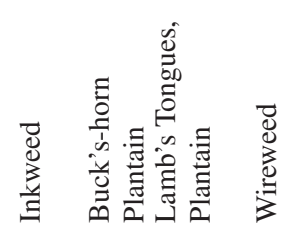

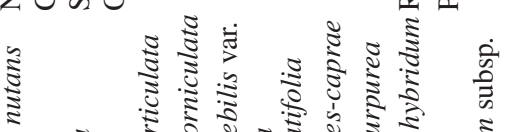

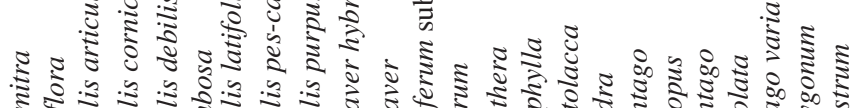

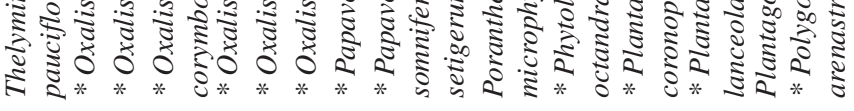
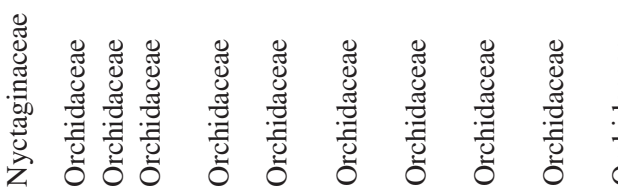

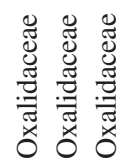
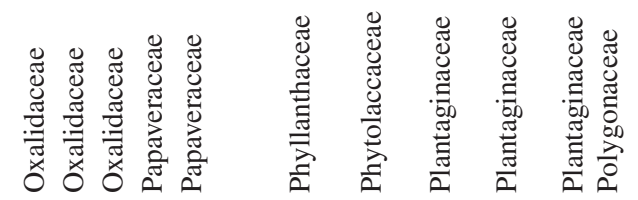

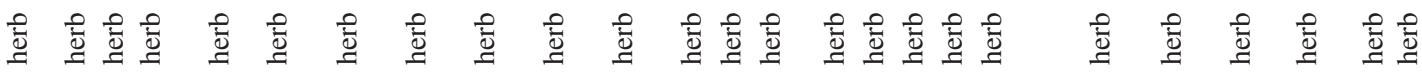




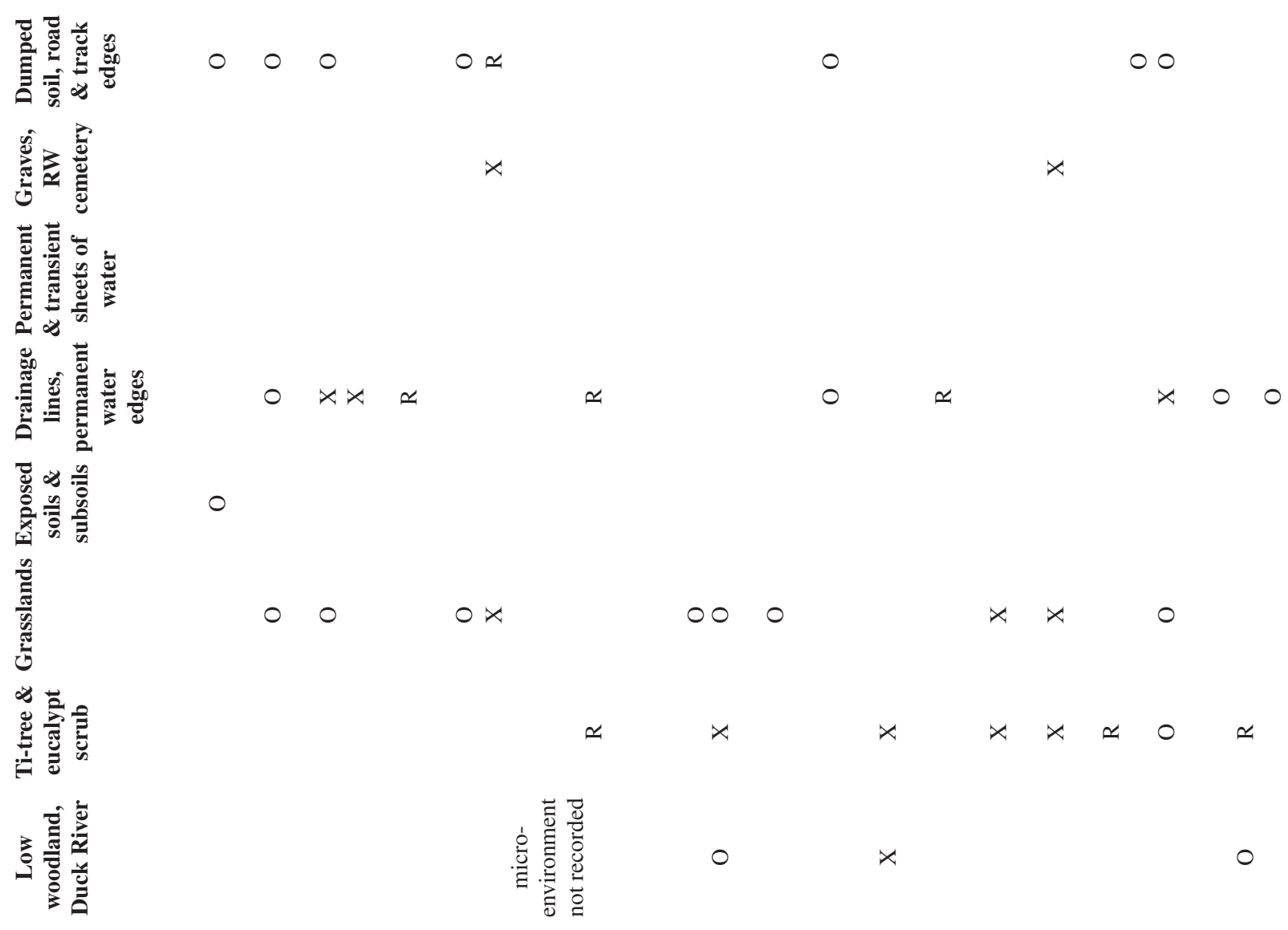

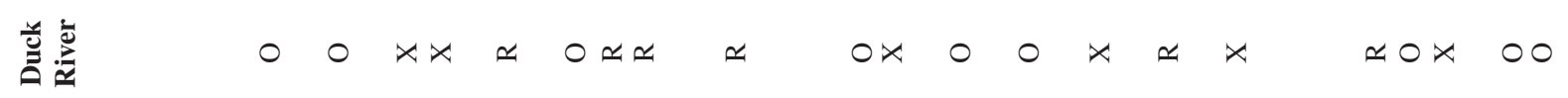

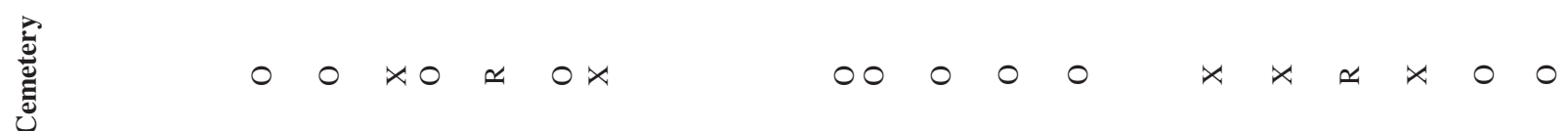

峞

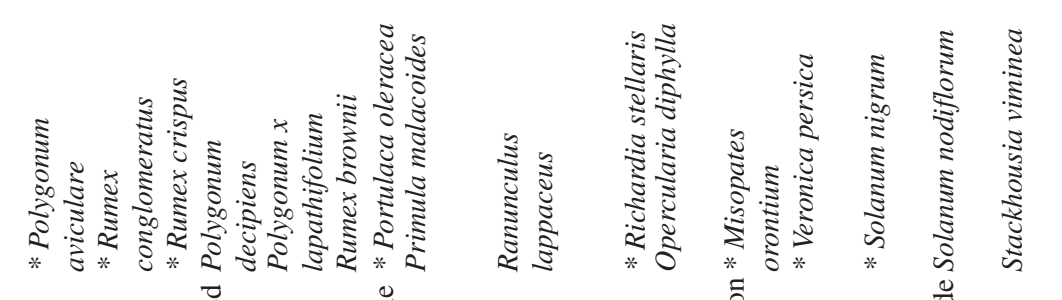

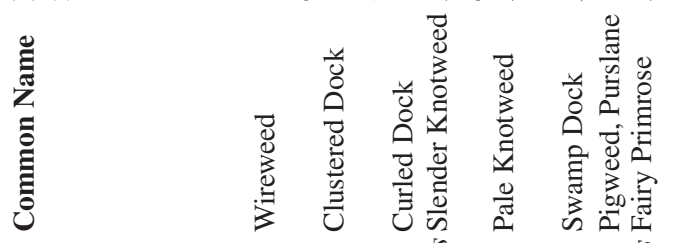

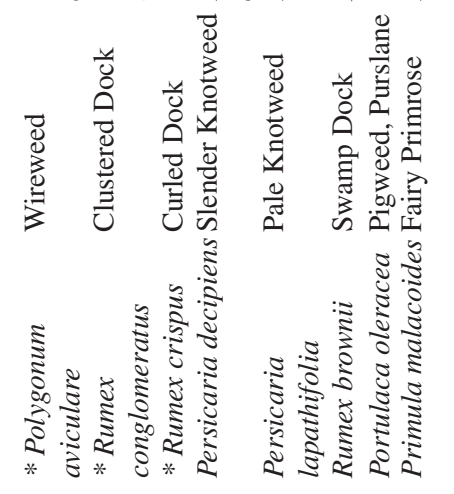

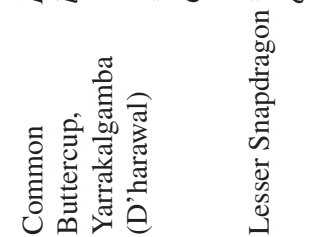

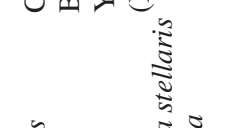

$\simeq \quad \circ \simeq \simeq$

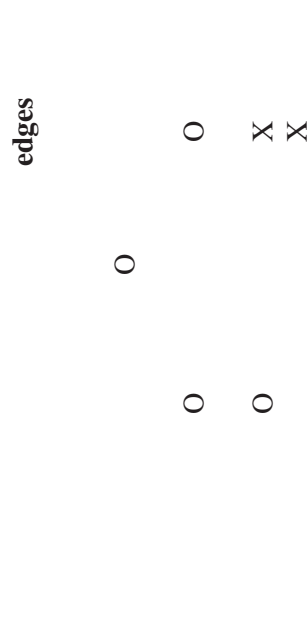

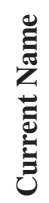

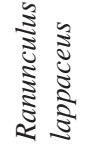

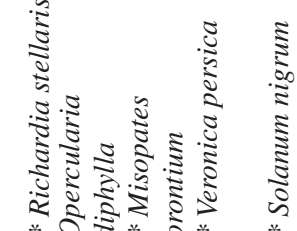

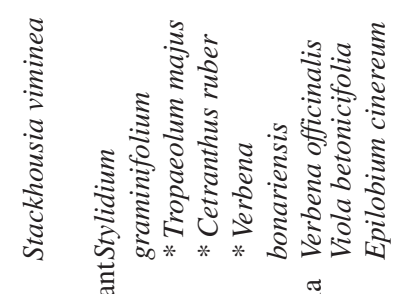

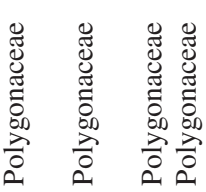

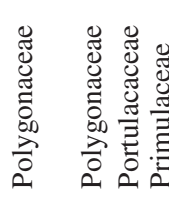

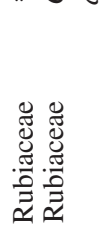

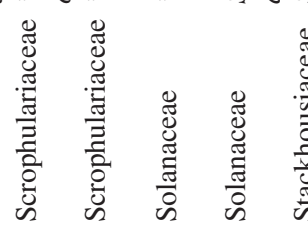

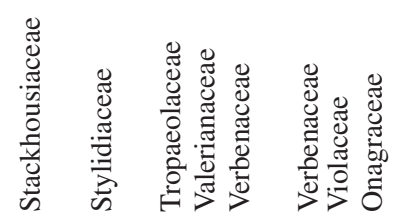

言竞

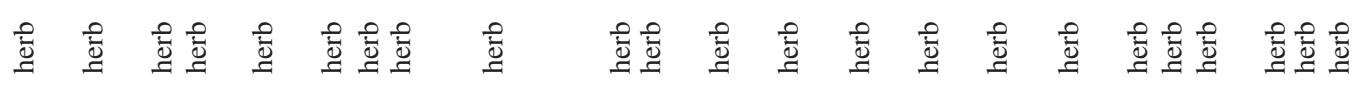




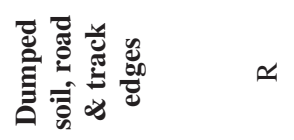

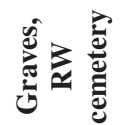

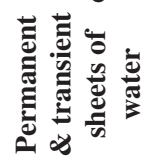

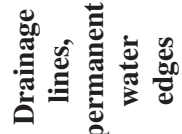

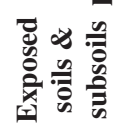

昰

$\simeq \simeq$

\%

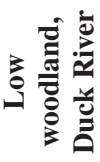

产芠

:

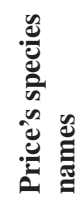
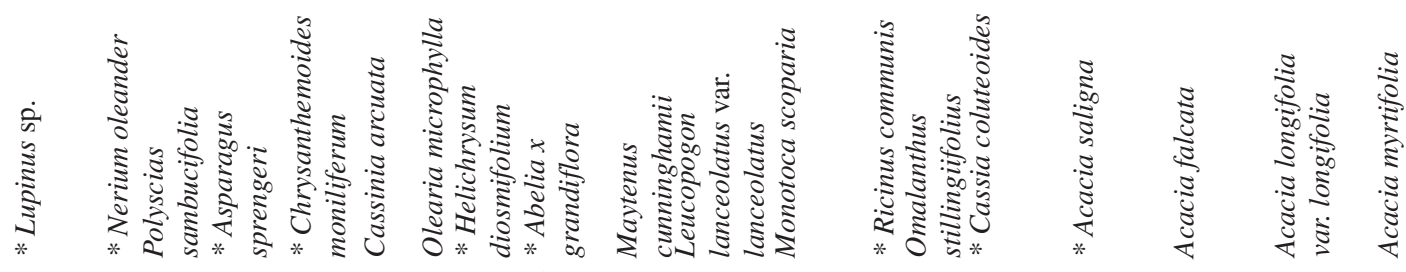

है

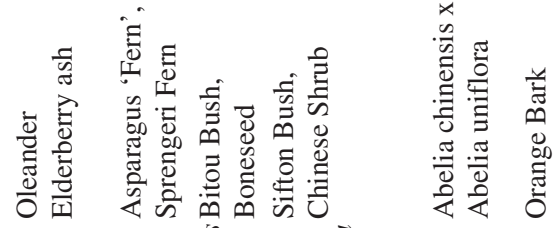

ڤ્छ

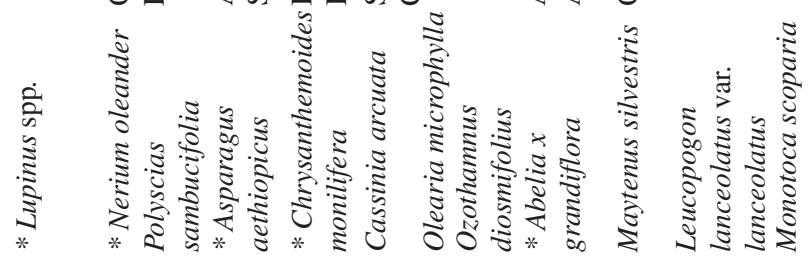

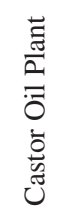

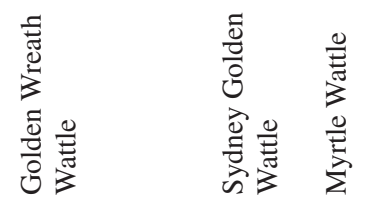

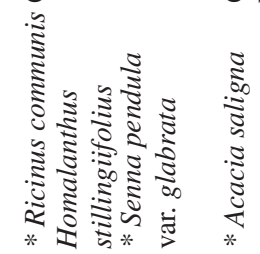

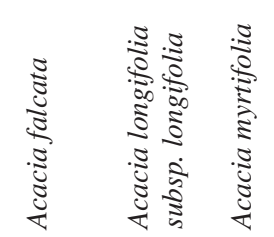

葛

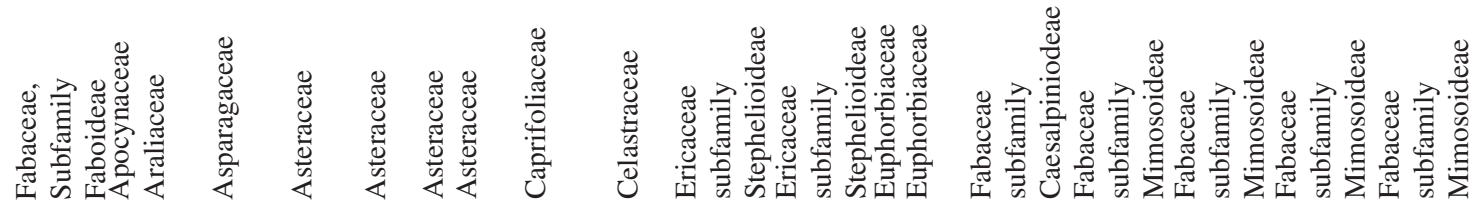

雀

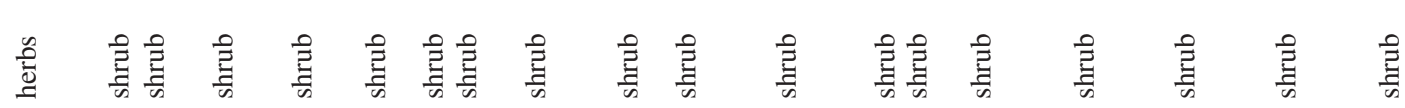




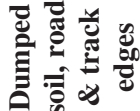

它范苞

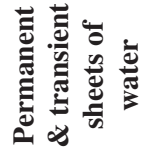

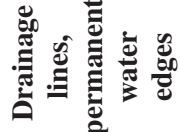

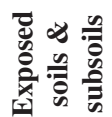

$\frac{\text { है }}{\frac{\pi}{\tilde{V}}}$

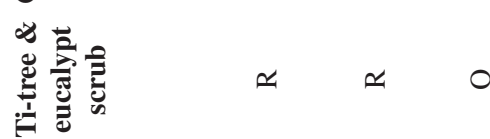

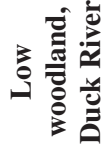

产高

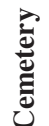

迅

葛

気

อี

U

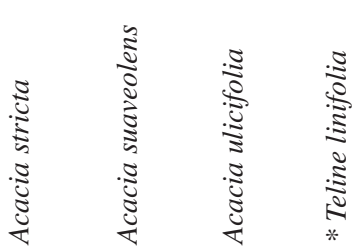

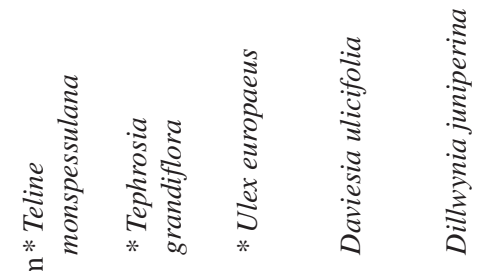

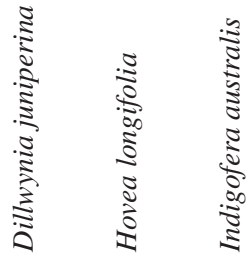

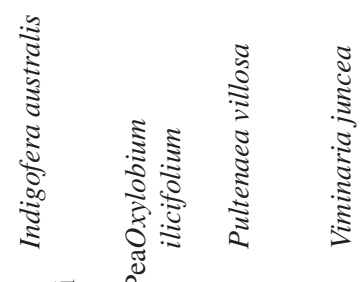

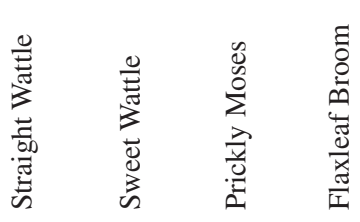

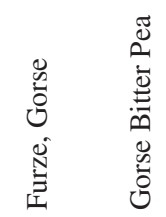

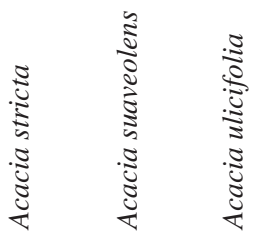

章

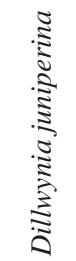

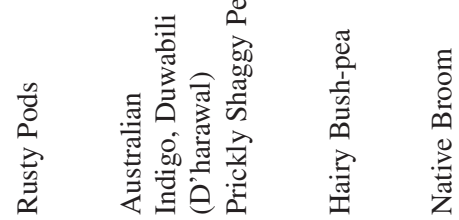

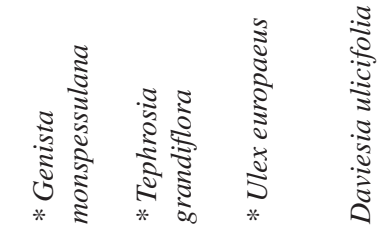

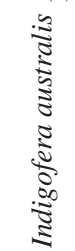

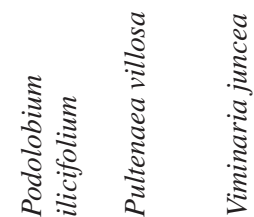

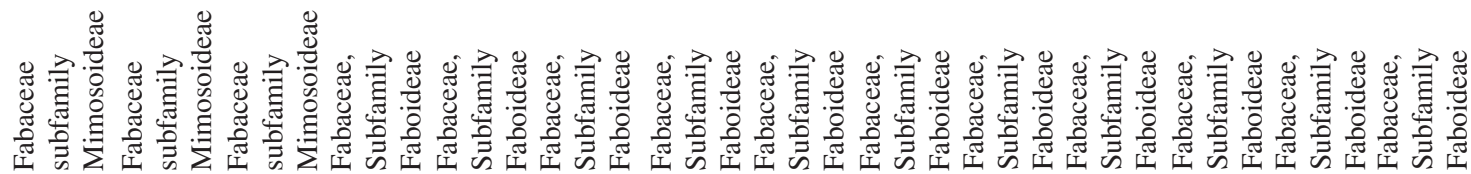

踏

章

毫辜

高

高

吊毫

重毫喜重高 


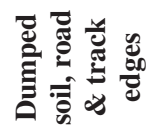

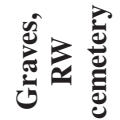

$\simeq \simeq$

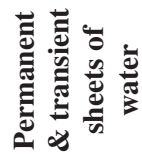

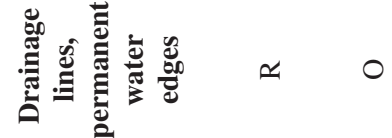

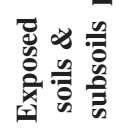

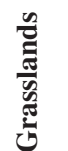

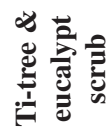

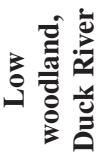

$\circ 00 \propto$
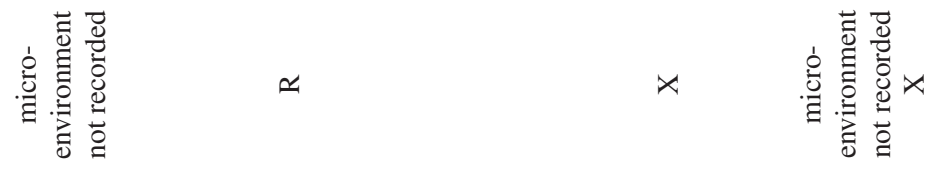

善衰

$\simeq 0 \quad 0 \simeq$

000000

$00 \times 00$

$\times 0 \simeq 0$

Uั:

$000 \simeq \simeq \simeq 0$ 0 $0 \simeq \times 0$

$0 x 0 \simeq x \times \simeq 0$

気

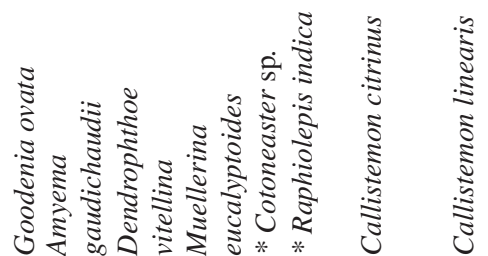

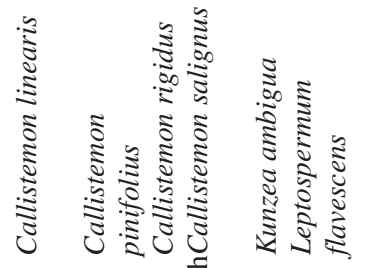

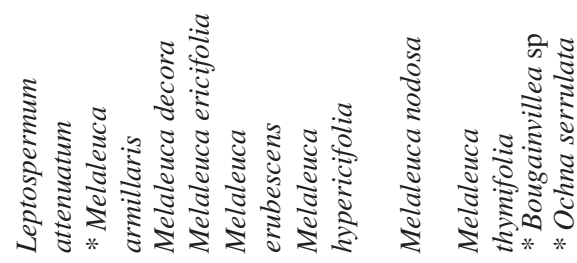

己ั

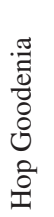

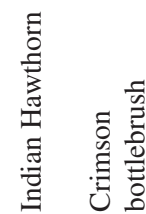

च

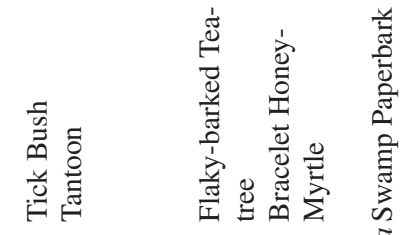

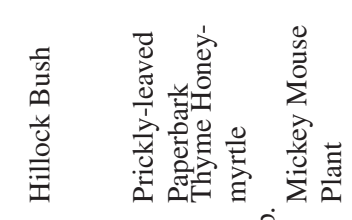

Uัّ

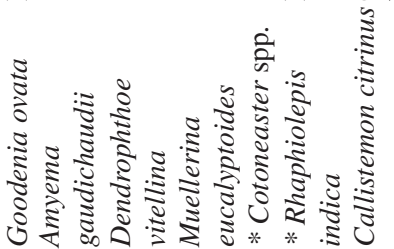

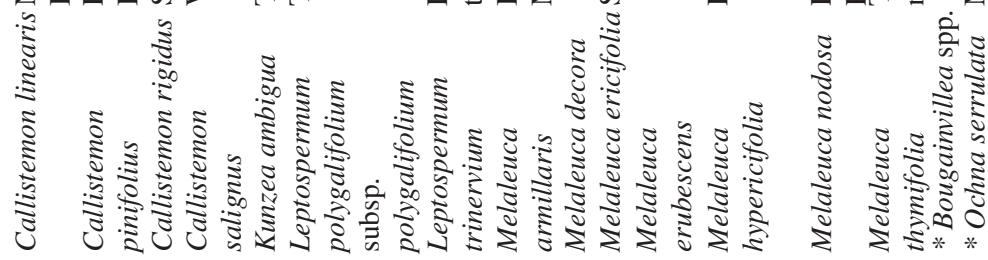

竞

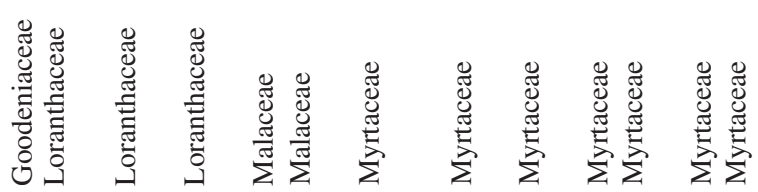

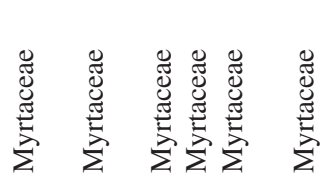

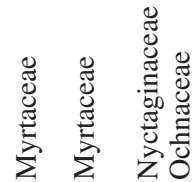

言音

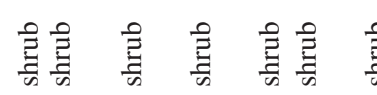

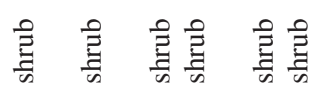

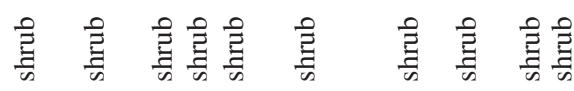




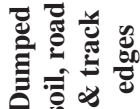

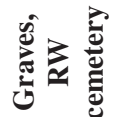

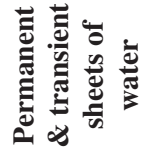

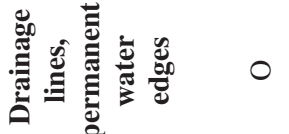

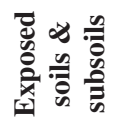

ט.

\&

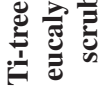

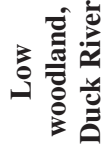

这

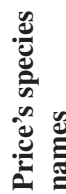

ถั้

שัّ

递

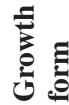

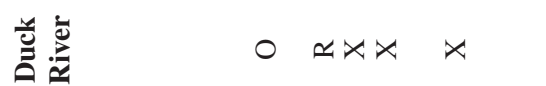

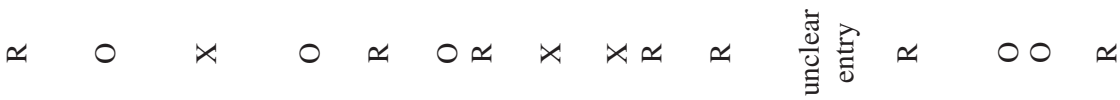

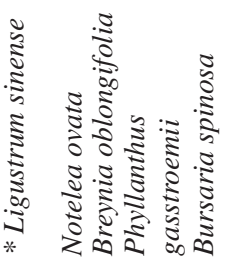

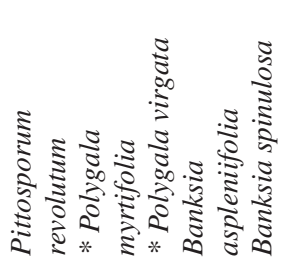

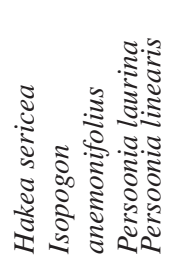

$\simeq \quad \simeq$

$00 \simeq$ $\simeq$

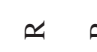

$$
\begin{aligned}
& \simeq \simeq 0 \quad \simeq \quad 0 \\
& \simeq \quad \simeq \quad
\end{aligned}
$$
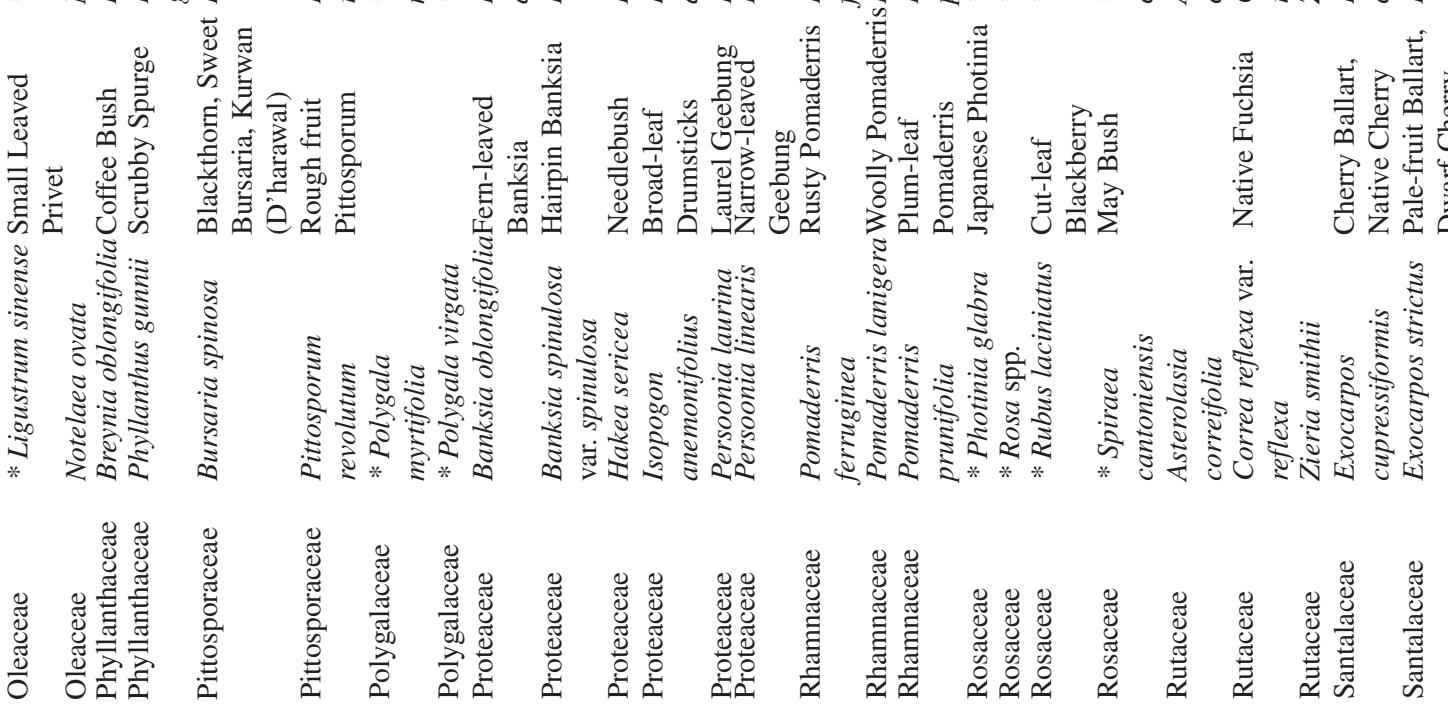

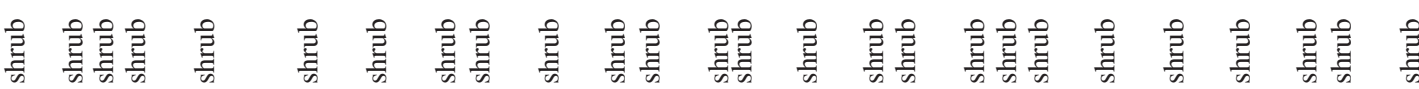




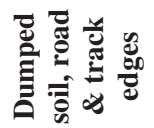

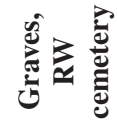

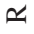

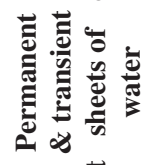

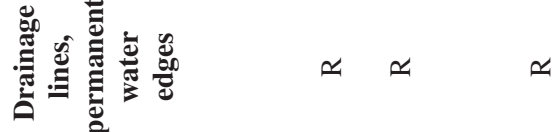

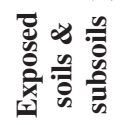

卷

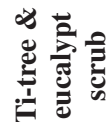

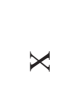

$0 \simeq x$

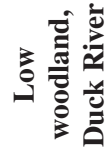

$x \simeq \simeq \simeq \propto x \quad \simeq$

$0 \simeq 00$

蓄

$x \simeq \simeq \simeq 0 x x 0$

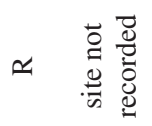

$\simeq \simeq \simeq \simeq$

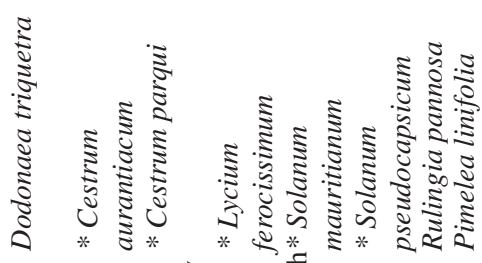

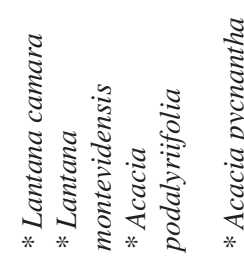

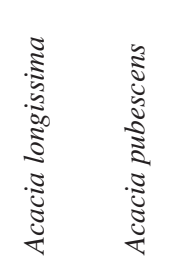

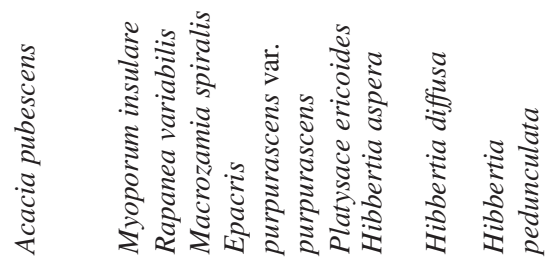

薝

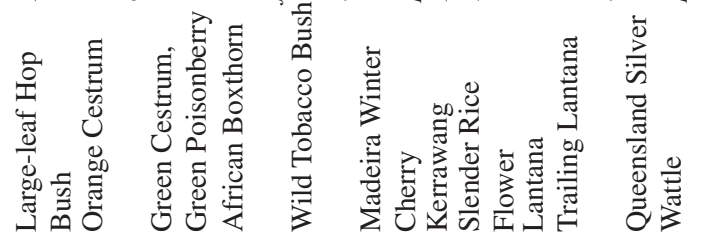

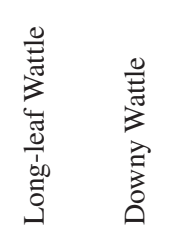

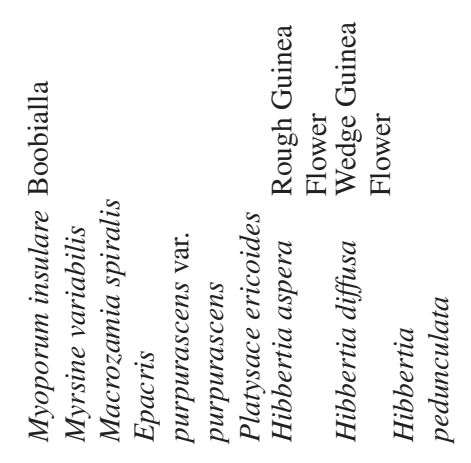

党

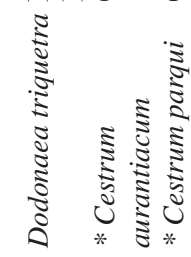
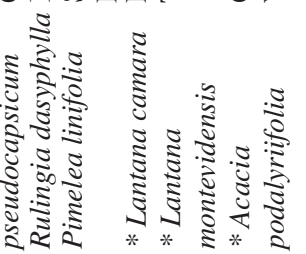

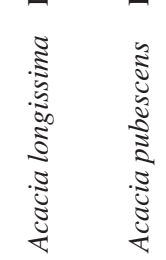

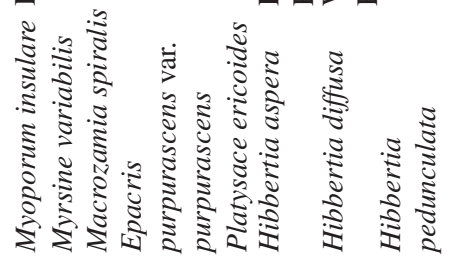

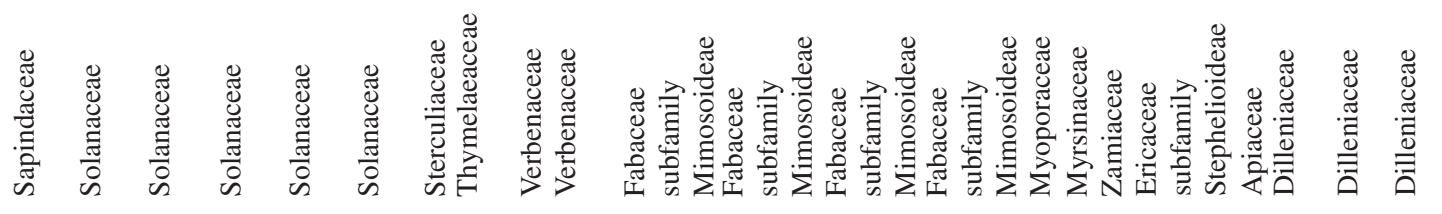

言竞

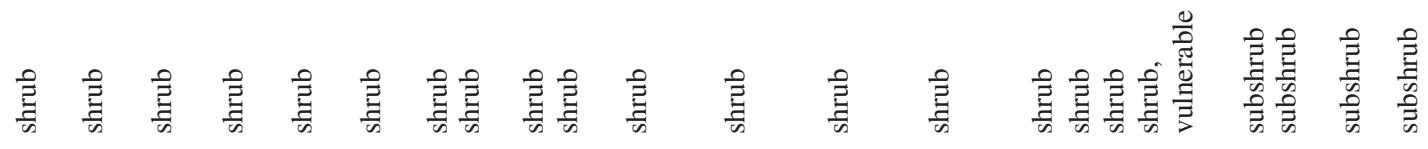




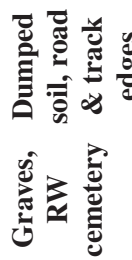

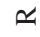

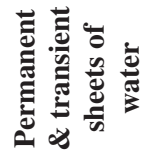

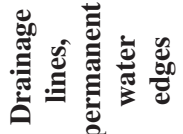

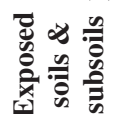

焉

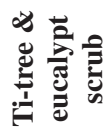

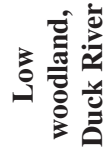

产产

Uัँ

कूष

递

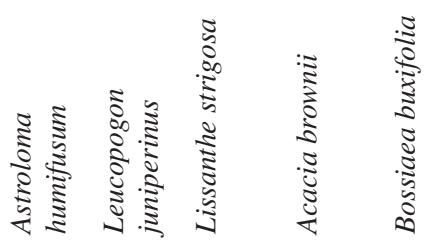

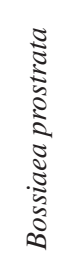

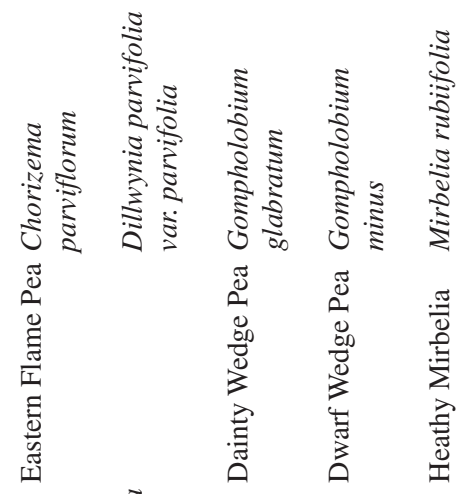

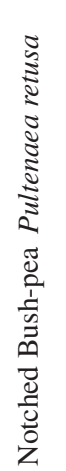

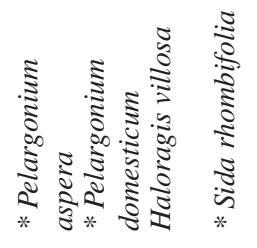

己े

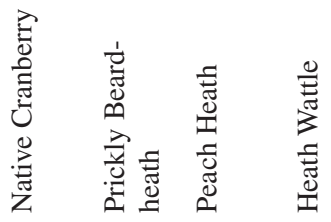

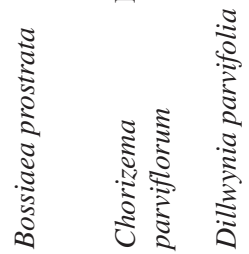

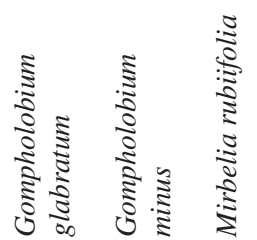

芩

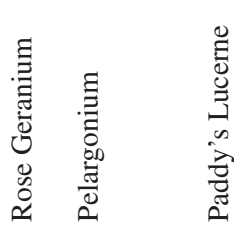

这

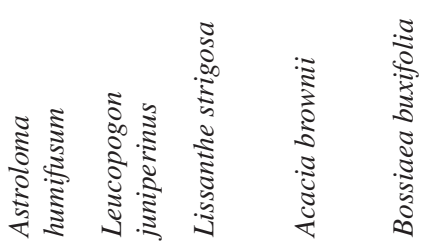

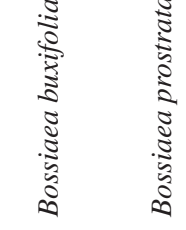

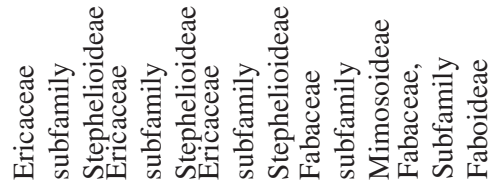

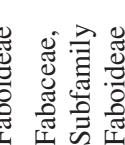

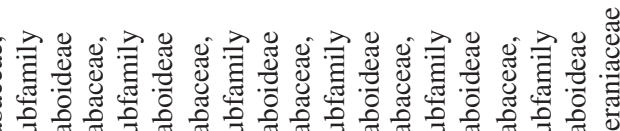

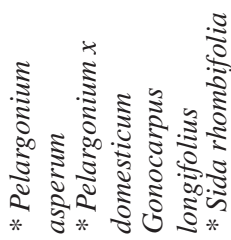

竎

㫮

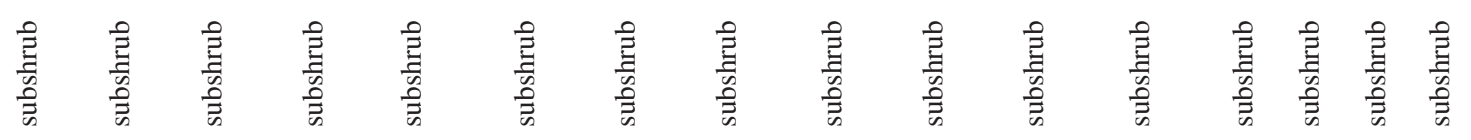




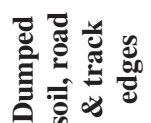

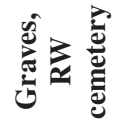

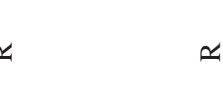

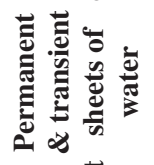

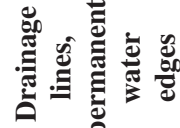

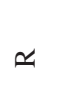

$\simeq$

$\simeq$

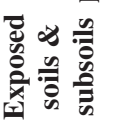

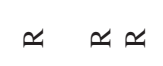

$\simeq$

离

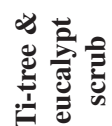

$\circ \times \quad x$

$\simeq$

$\simeq \propto \propto ⿻ \propto ⿻ 上 丨$

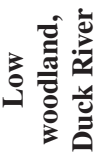

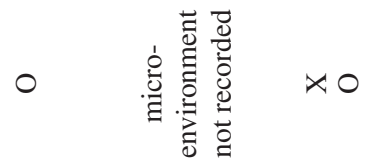

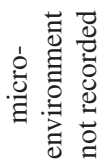

苍

$\circ$

$\simeq \simeq 0 \simeq x$

$\simeq \simeq \propto x$

$\simeq \quad 0 \simeq$

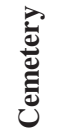

$\simeq \simeq \simeq$

$\frac{\mathscr{s}}{\tilde{x}}$

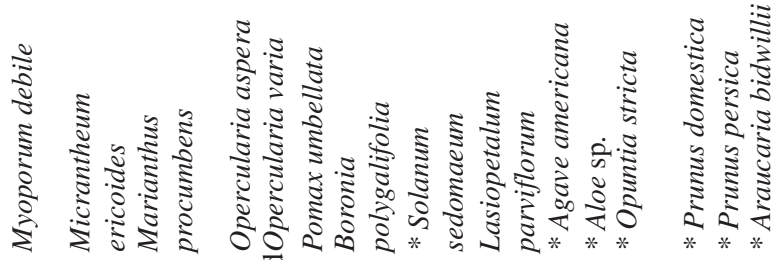

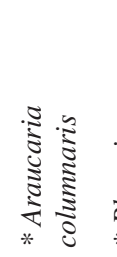

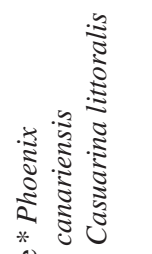

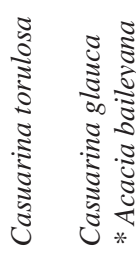

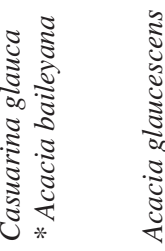

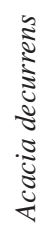

己े

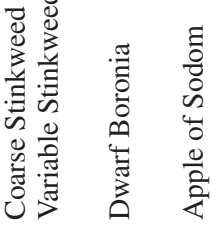

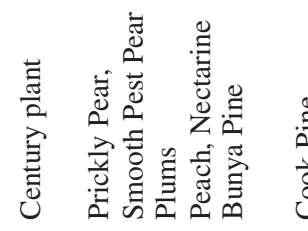

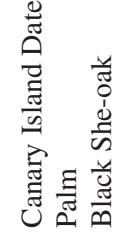

范

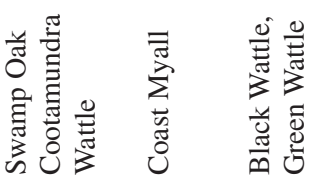

崖

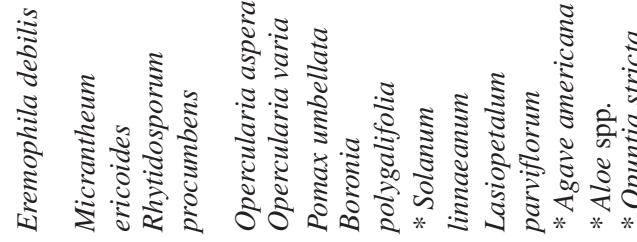

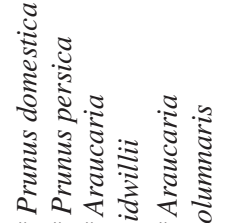

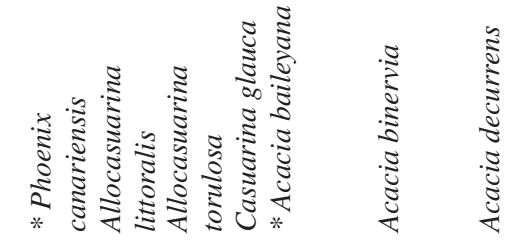

离

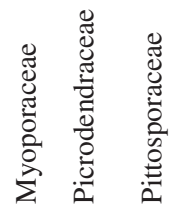

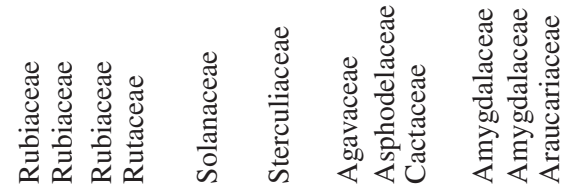

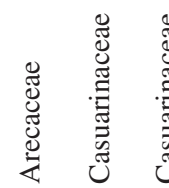

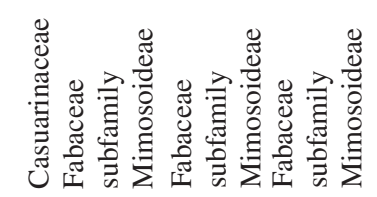

紊罗

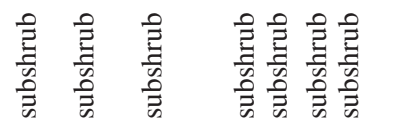

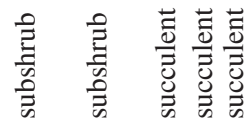

: 巳:

导导导导导导 


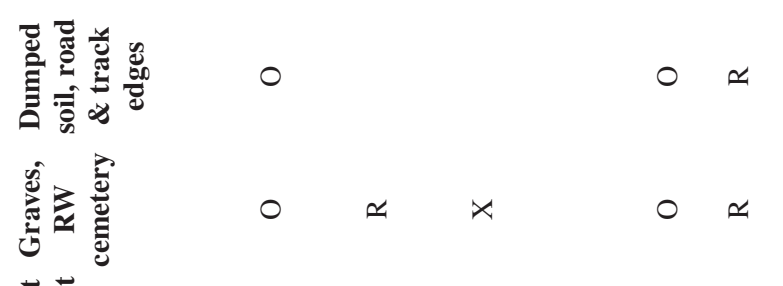

蓠苛

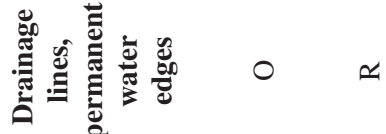

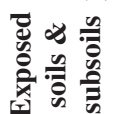

ט.

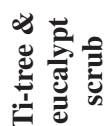

$\simeq \simeq$

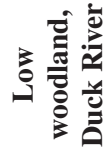

$\simeq$

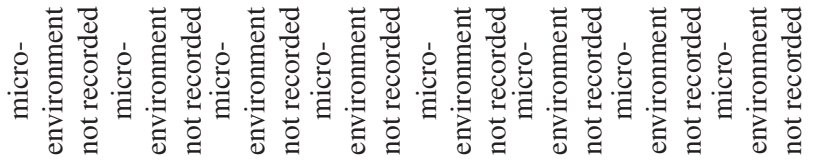

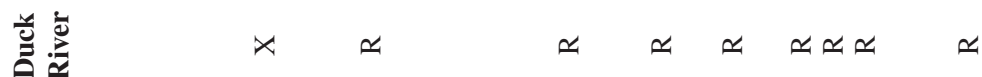

这

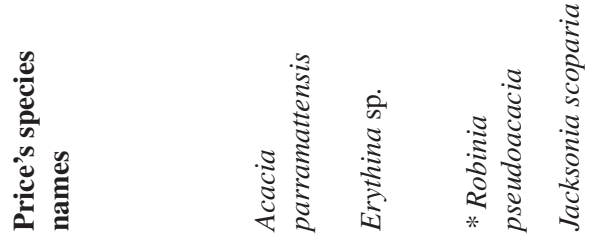

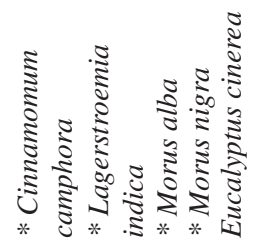

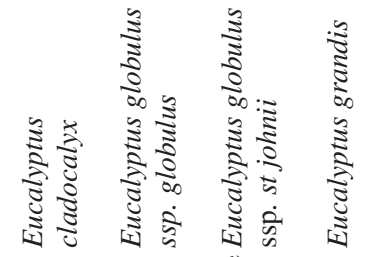

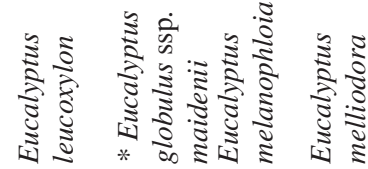

节

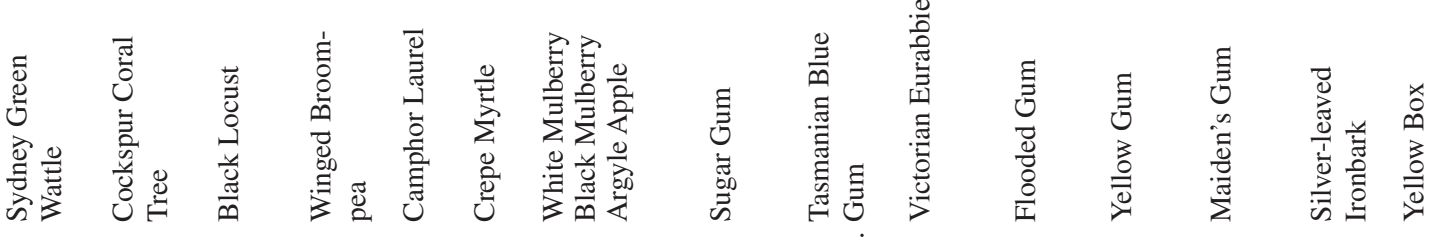

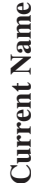

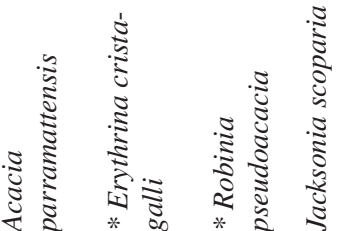

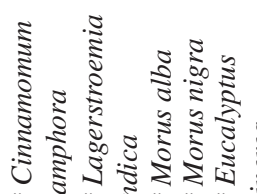

害

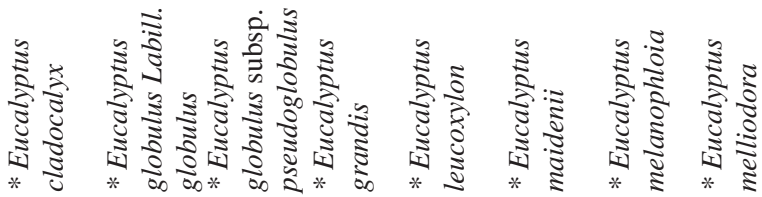

吾

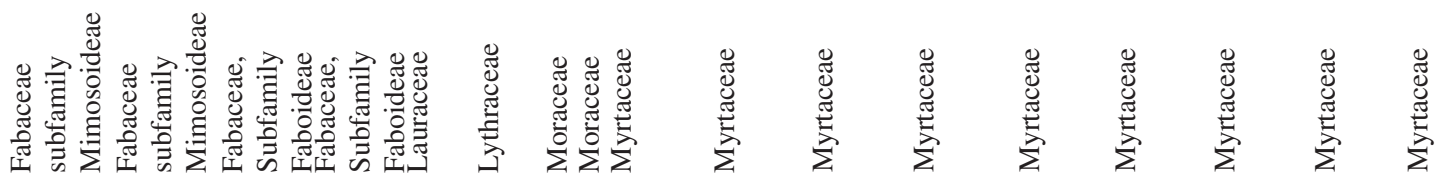

竞跑

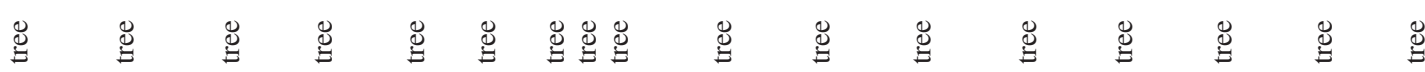




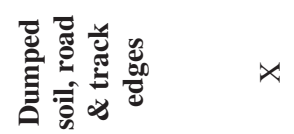

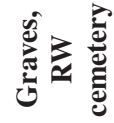

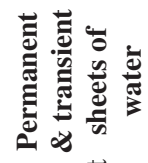

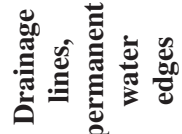

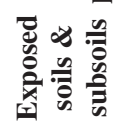

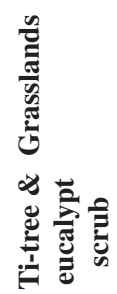

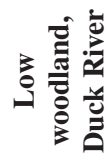

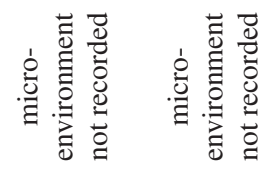

亭

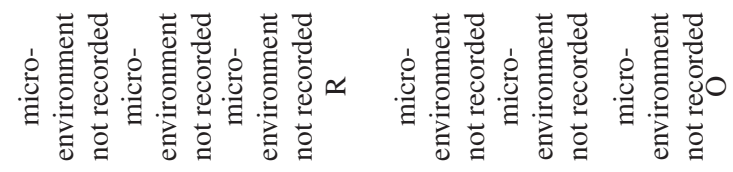

蓄

I

边

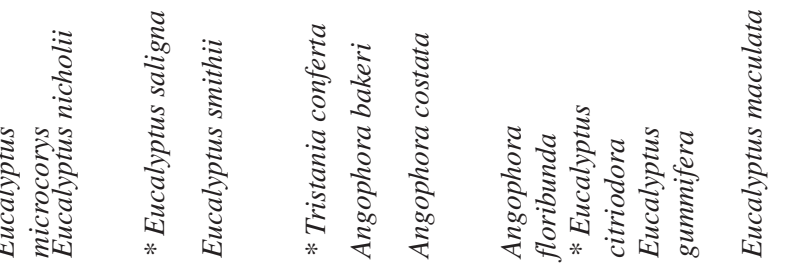

离

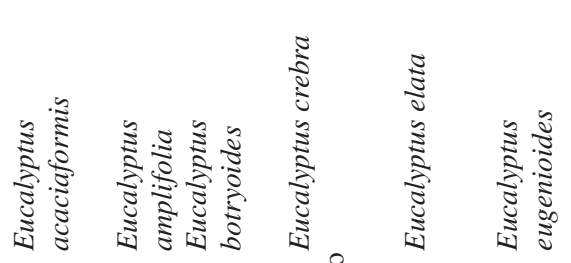

嶽

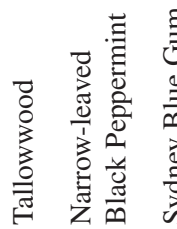

言

(1)

I. the

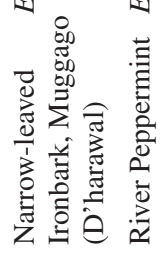

I

I

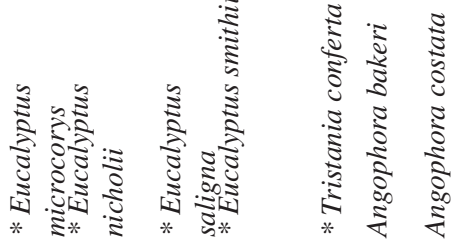

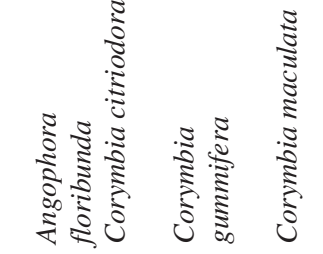

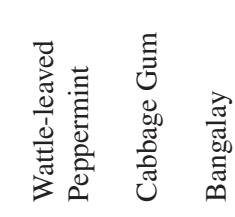

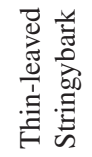

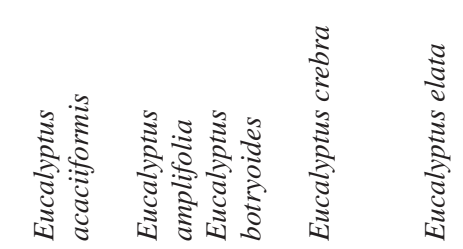

善高

I

$\|\Pi\|$

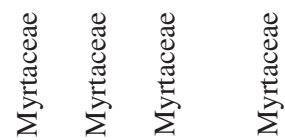

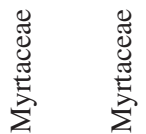

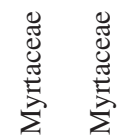

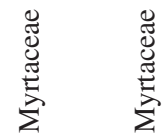

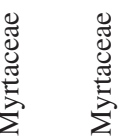

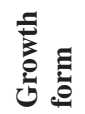

๕ 


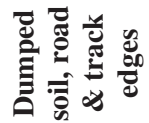

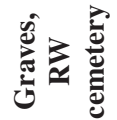

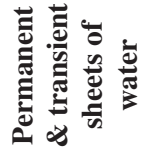

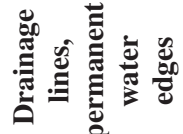

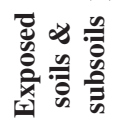

ט.

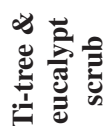

00

○

ق气

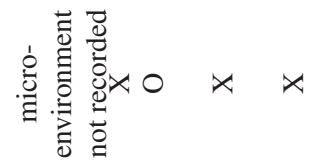

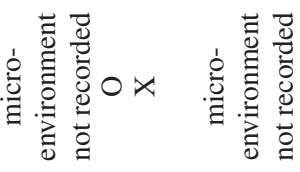

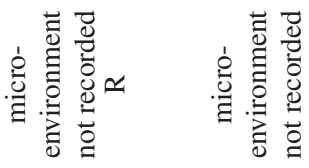

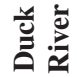

Uัँ

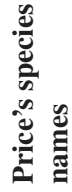

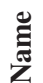

音

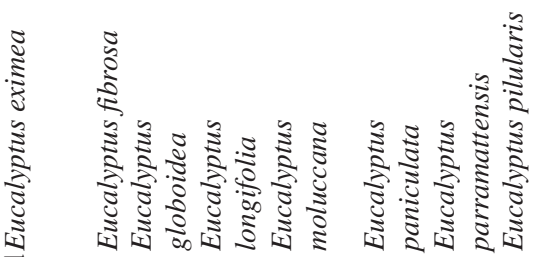

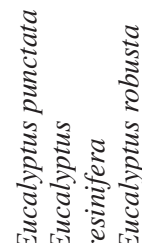

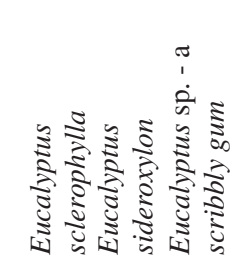

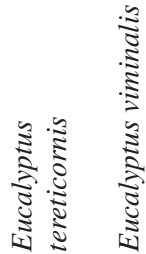

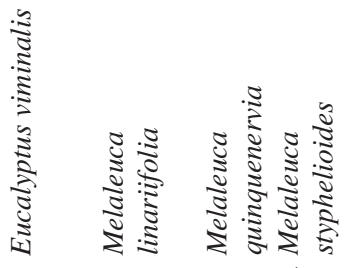

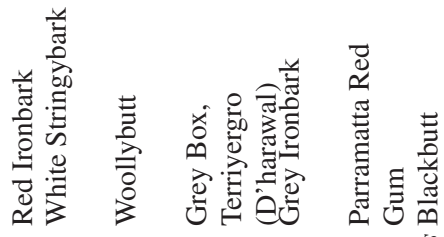

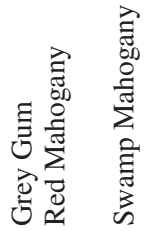

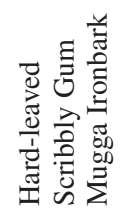

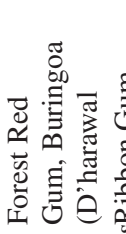

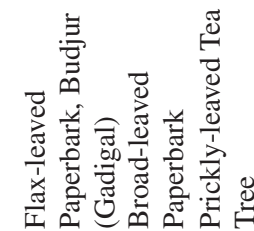

己ัّ

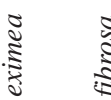

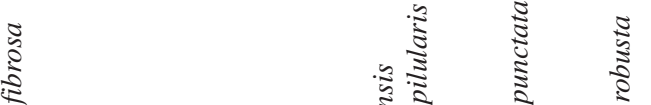

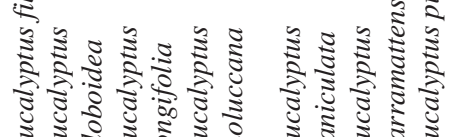

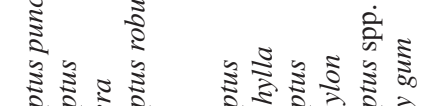

药

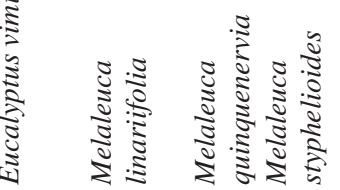

竞

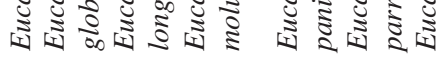

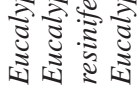

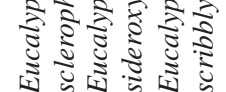
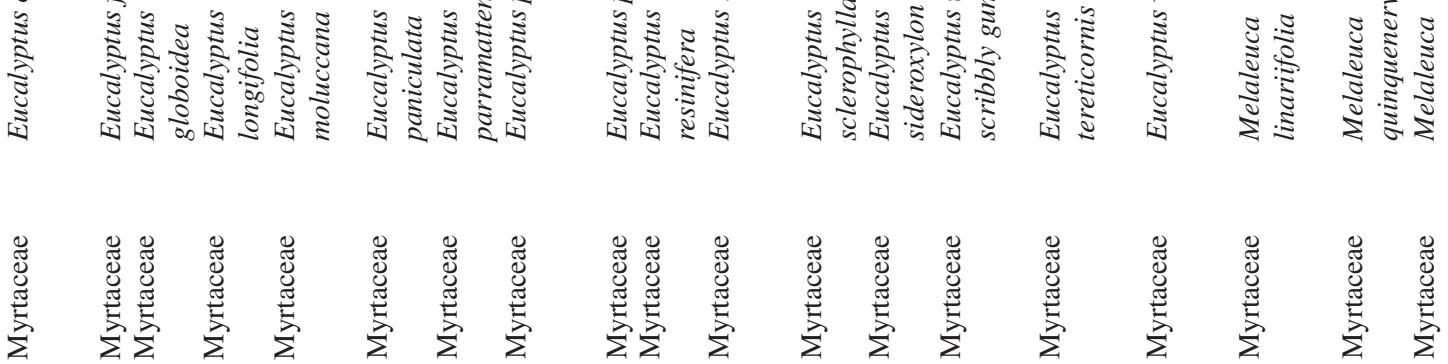

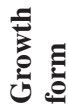

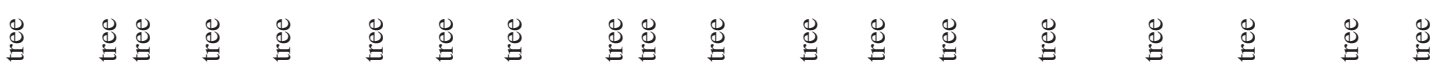



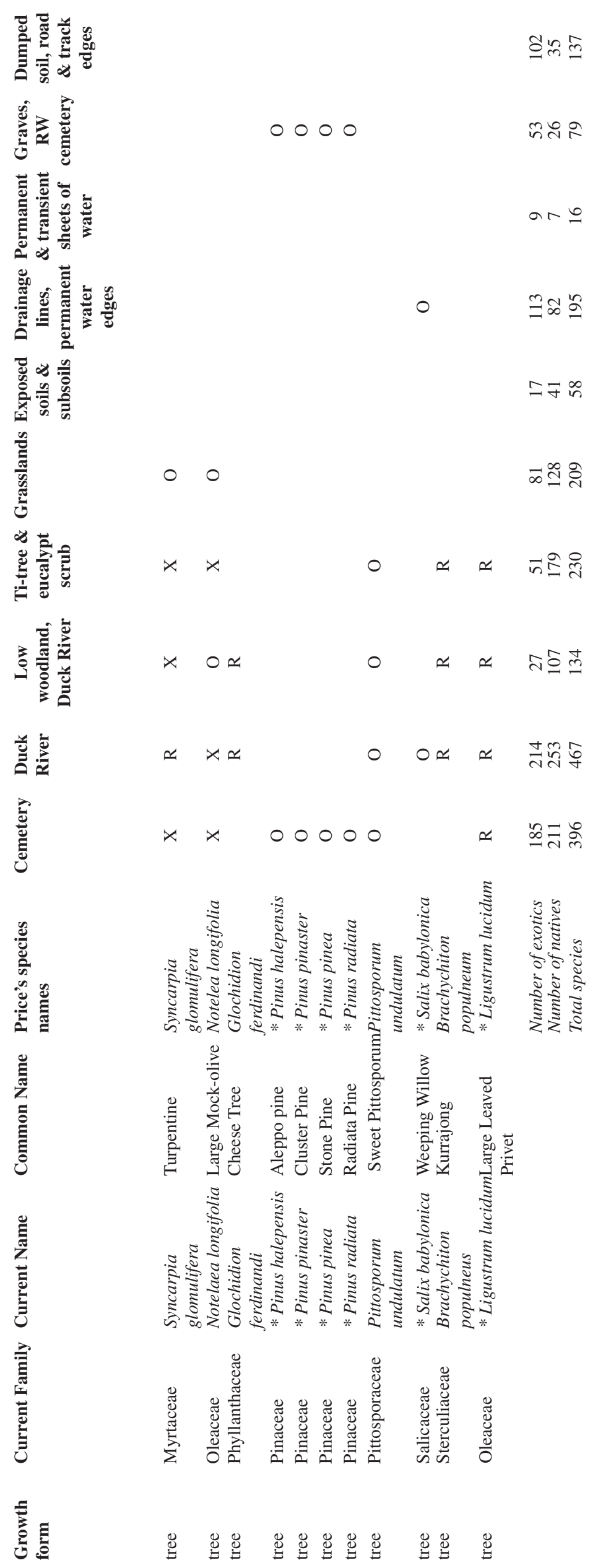
Appendix 2

\author{
The Veretation of Duck iriver and \\ kookwood Cenetery, Aueurn \\ (with a liat of specios)
}

i.h. Price

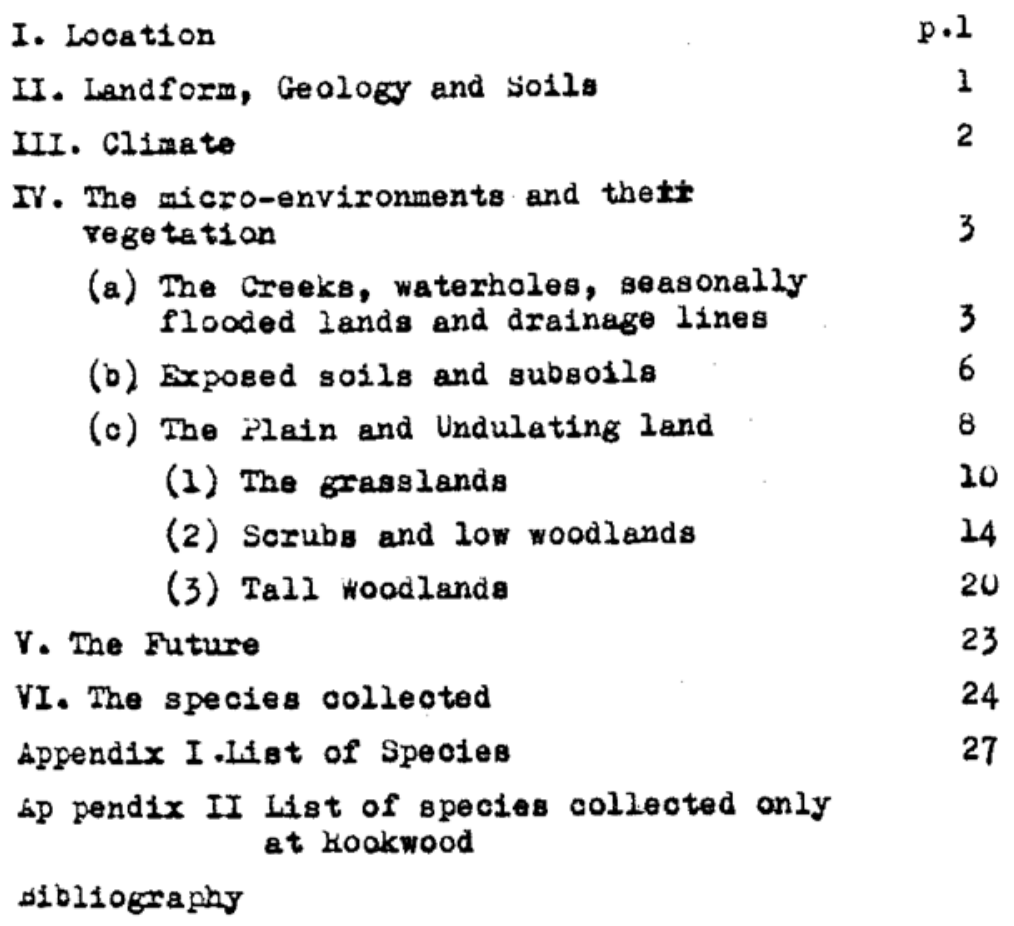

hap I (Included in text) Location of sample areas

kap II (at rear) Iruck and Haslems Croëks

Lap III Inck River sample area (at rear)

Kap IV kookwood sample area (at rear)

The argument: The Auburn area carries the remants of a much diaturbed Ilora representative of that normally found in the wetter parts of the W ianamatta shales of the Cumberland Basin but there is Elso the remnantx of a sandstone flora, particularly at kookwood. The original type of vegetation was probably a tall woodland or dry schlerophyll forest in which the dominant trees were those of the drier parts of the Cumberland basin (an bucalyptus moluccana-tipfibrosa sap. fioroga) sesociation) but a number of the aubordinant tree species and many of the understorey plants were species of moleter environments. There are no longer any stands of the original tall woodlands and the dis turbed vegetation has been shaped largely by man and fire into Brassiands (dominated by either native or exotic associations of grases and herbs), ti-tree and euoalypt scrub (with kunzea sorub a variant on exposed sub-solis) but in one area the sorub has evolved into a low roodland. 
The Veretation of Huok kiver and

kookwood venetery, Auburn

G.....ren

I. Dobstion

The perts of the hookwood Cemetery and the buok kiver which have boen surreyed are in the mid w-western suburbs of bydney 12-14 miles w.S.H. of the city (see Wap I). Both are part of the southweetern portion of the Ferramatta KIver Hagin butthe southern end of the rookwood ceactery extends over the divide between Haslem's and the freshwater weeks into the uppex reaches of the Coaks kiver.

II. Lendform, Geology and Solls

The Duck biver and Haslen's (reek basing (see map II) form a V-shaped wedife of country that pivots in the south east on the aandstones exposed 1n the bigh country about $r$ otte 4111 by the warpine and differential ersoion that has helped form the Clumberland basin. Untliers of sandstone-like rocks occur north of rotts Hill at such places as ini2l1pe Hill (not seen) and the iresbyterian burlal sround near veorges irenue (a silicifled alztetone). The Duok kiver has also out down Into a quite large stratum of aandstone that underlies and extends some distance beyond the ourvey area there. Hut none of the sandstones north of rotts Hill is massive, each apparently having a quite high clay content, and none has any obvious effect on the shape of the land. Ihe cominant bedrock is the soft, easily eroded shales of the lilanamatta Series that are so deeply weathered that they are exposed only in Ieilway cuttings (Kookwood, Hegents Park, Ghullora) and so littlo apore oase level that the land is nearly flat or gently undulating.

both basins are poorly defined by gentle undulations little more than forty metres above sea level. The broad, almost flat plain rising Fadually to the east and west and imperbeptioly to the low undulations thet still out aoross the oreeks' courses lo largest on Luok kiver and encompesses the whole of the survey ares there. Ihe area in the fookmood cemetery and the old state Hospltal grounds is nearer to or onthe southern and eastern watershed of Haslem's oreek so the country ia more undulating. However, fffors of flat land extend well upstream into the cemetery from Lidoombe and are broader in the Carnarvon lolf lourse (once part of the Hopstialal). The buok Hiver seems to have had a permanent flow these past three years though often it has been little more than a trickle of factory effluent and atreat drainage though that is pure enough these days to support freshwater fish and tortorse and the oreeding of duoks on the deeper, proader reaches. Nonetheless, 'the river' above its tidal basin is simply a storewater ahannel for all that its natural banks have been kept in Auburn and liranvilie. Its 1 erel can change abruptly and flooding nay occur below hhlsolm hoad after heary storms once the oatchment has become saturated. Haslein's lreek Is a series of atormwater channels that flow after rain, even the most 


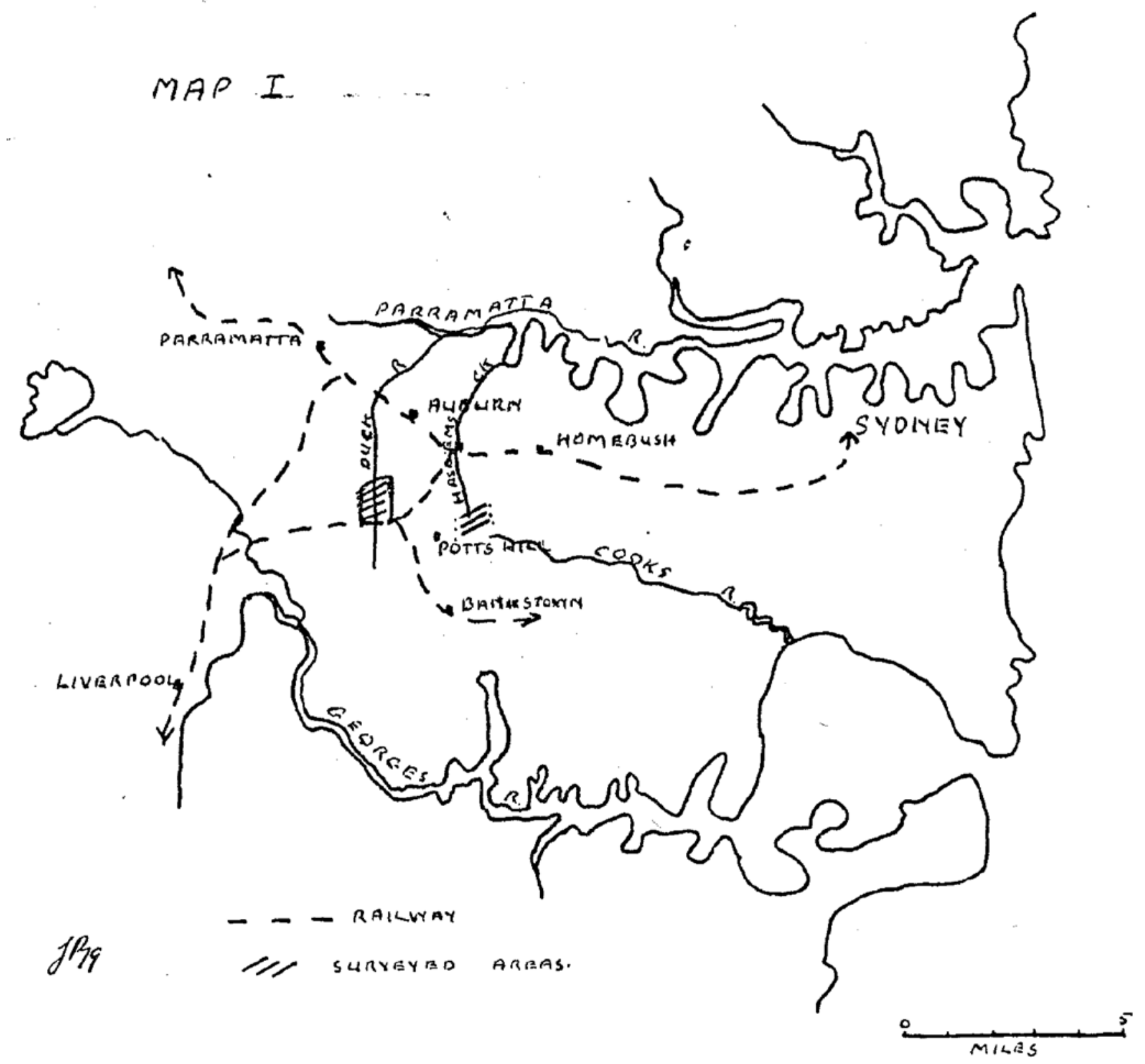


ephemeral oulles in the state Hoppltal grounds and the lemetery having oeen bricked.

Solls everywhere seem to be deep, as muoh as up to $81 x$ to elight feet on even the Juck Liver sandstones, and seeln always to be underlain by much decaying rock. To the eye, topacils seem allike whatever the basefrock and are a grey, vertically jointed, podsol-like ' $A$ ' horizon up to some 12-18 inches deep that protects a softer, more easily erodaole subsoil. Subso1la seem mare variablex in colour and texture but seem always to be clays - deep, tough, elastio red clays at 296 Fark koad and on the $2 x$ northern sloper of Freshwater lreek, a yellowish-white and somewhat more orumbly clay once much valued by looal tile and pipeworke on the Juck Hirer sandstones. Sinoe alithe so1ls seem to have a high olay content they a.re prone to crack widely and deeply in exposed places in dry westher, many of these fractures apparently persiating for years (Duck kiver, 296 Park hosd) and sonetimes become accentuated oy erosion to give a peculiar raised and blook-1ike appearance to the surface. In wet seasons all soils become saturated and most water runs off the surface rather than percolating into the subsoil. Pertility 18 moderate though I have not had any samples analyoed. Yet, for all this apparent uniformity in the solls the remants of a sendstone flora oocur in the headraters of Haglem's creek and at Potts Hill but are legs well represented on the Duak Klver. However, most of the species colleoted are those that are normally found on w ianamatta shales.

\section{III. caimate}

The average annual rainfall and tempeirature figures (seo pase 3) are those of an area with mild wet winters and warm wet suminers and put the sample area towards the milder, wetter end of the specturm of climatic change that occurs from east to west in the sydney basin. kainfall is a ufficiently high for there once to have been a 'tall woodland' or 'forest' yet sufficiently varisole, given the nature of the soila, for

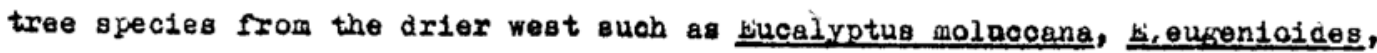
E. parramattensis and E. longlfolla to mingle with others from wetter

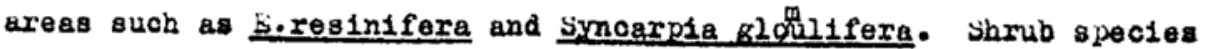
also range from jlants suoh as Kunzes amoisua, pultenaes villoga that brow well only in open, somewhat exposed situations to others such a: Gloohidion ferdinandi, Breynia oblongifolla and Notolaes longifolia which are normally plants of wet, shaded foresta.

However, average figures of the sort given above are often less laportant for plants than the extremes they mask. bven over the past finger yearg rainfall has been aufficiently variable for the totala for the years to be roughly 'average' though the soils have veen waterloged for months on end (as in the winter, spring, and early sumer of 19\%) and then to have gradually dried out and cracked deeply (late sumer, early autumn of 1977). bixposure to sun and wind, perticularly to the hot dry 
$-3-$

norti-westerlies in summer and cold dry south westerlies in winter con compound these problems of soil wolsture and the streas that they can wow cause in plants. In the sprinb of 1977, for example, so 118 weis deeply $c$ cracked and even the oufhest of herbs and srasses were veginning to fail where the aspect was north westerly one without scelter. Yet scarcely fifty jerde eway in sheltured woodland soft species such as the natden bair tern (Adiantum aethioploum) were growing well in a topsoil which was atsll moist. Probably sirilar variations in temperature extremes can occur. Frost, for expmple, setme only to oocur in open graselancis at Ducix alver and trees and shrubs there provide a freat deal of sheltar from chill, outherlies in winter.

The surrey was made during a cyole of wetter than everage years and it may be that during droughts some of the plants adapted to moiater enviroments inight fail as the one colony of pomaderrig ferrubinea an Drack Hiver almost did during the dry spring of 1977. However, there roul a robably always be molstex, more sheltered niches in which species night survive or re-establish. Of course, it may de that at least some of the aberrant distributions given in the species list owe litile or no thing to changes in rainfall and are due ojmply to the disturbed ecolosy of the pushland axeas about Sydney and the changes in the havits of migratory birds and 80 on that this has ouased.

\section{The micro-environments and their vesetation}

(a) Ihe oreeks, meterholes, sesonally flooded lands and drainase lines Tor persanent and trangient sheets of water

Only in the deeper reaches of the Luck Hiver is water jermanent and deep enough for there to be aoft, quite delicate out as yet unidentified woter plants which ourvive despite repeated, quite violent flushing during every heary fall of rain. Soft but pliant knotweede (polyzonum deciplens, P. lapathifolium) and alligator need (Altermhinere philoxioldes) rooted in the Danks about low water level also aeem able to regenerate after flood from a straggle of atripped stems by the following orowinis aeason, the alligator weed so successfully that it takes over much of the water surface by mid summer deapite the cSInv's control programme.

The areas of semi-permanent and tranglent aheets of water are equally dificult environments where changes in the depth of the water, the lenoth of time it persigts and the atrength of 1ts flow, if any, can oe criticul for the plants that grow there. Hull-rushes (Typhs oriental1s) grow wezl In the dams on Carnarvon Golf lourse and the semi-permanent pond in the old shallow olay pit behind the woolworth's factory on luck kiver. A succesior. of wet seasons has also allowed quite small colonies to spread onto a few sites that are simply moist but the river in flood 18 too violent for them to have oolonised the normally still waters behind the rrinces load weir. Sagbitaria (Saptarria graninoa var westhemana) has also adapated to the changting levels of the water in the buok kiver clay pits. 
$-3+-$

jata relating to ilinate

Average figures are not published for stations thear allourn, bo wose f'or syuney $(12-14$ iles to the east and by thesea) end farremattes ( 4 milea to the *est) are oiven.

line 1 sterage bally maximum teinperature in dogrees finreniselt

line 2 average daily winimua teijerature

ine 3 sverase daily inean temperature

line 4 iverage index of mean relative husality

ine 5 averase daily s p.m. relative hunidity

line 6 everage monthly and Yearly rainfall in inche aud pointa

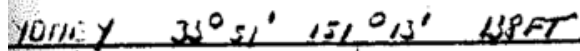

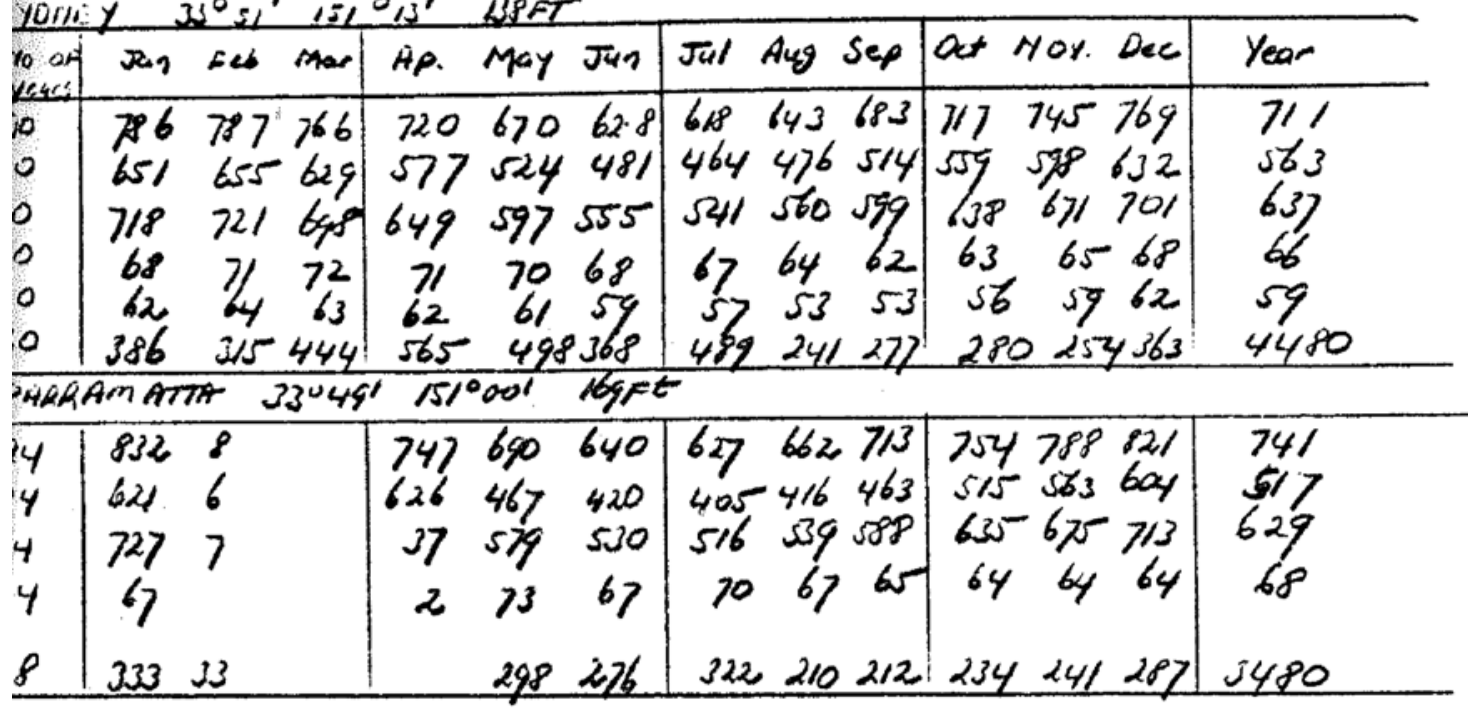

The mantily reinfall received and the number of days on which rain fell are aveilable for the Clyde Wagon Waintenance Works (roughly the crossing of the rain 'iestern Kailway line on buck kiver) and the rotts bill keservotr. ioxever, rainfall figures are in millinetres. The averase annual reinfell at Clyde is $994 \mathrm{~mm}$. that at fotts $4131909 \mathrm{~mm}$

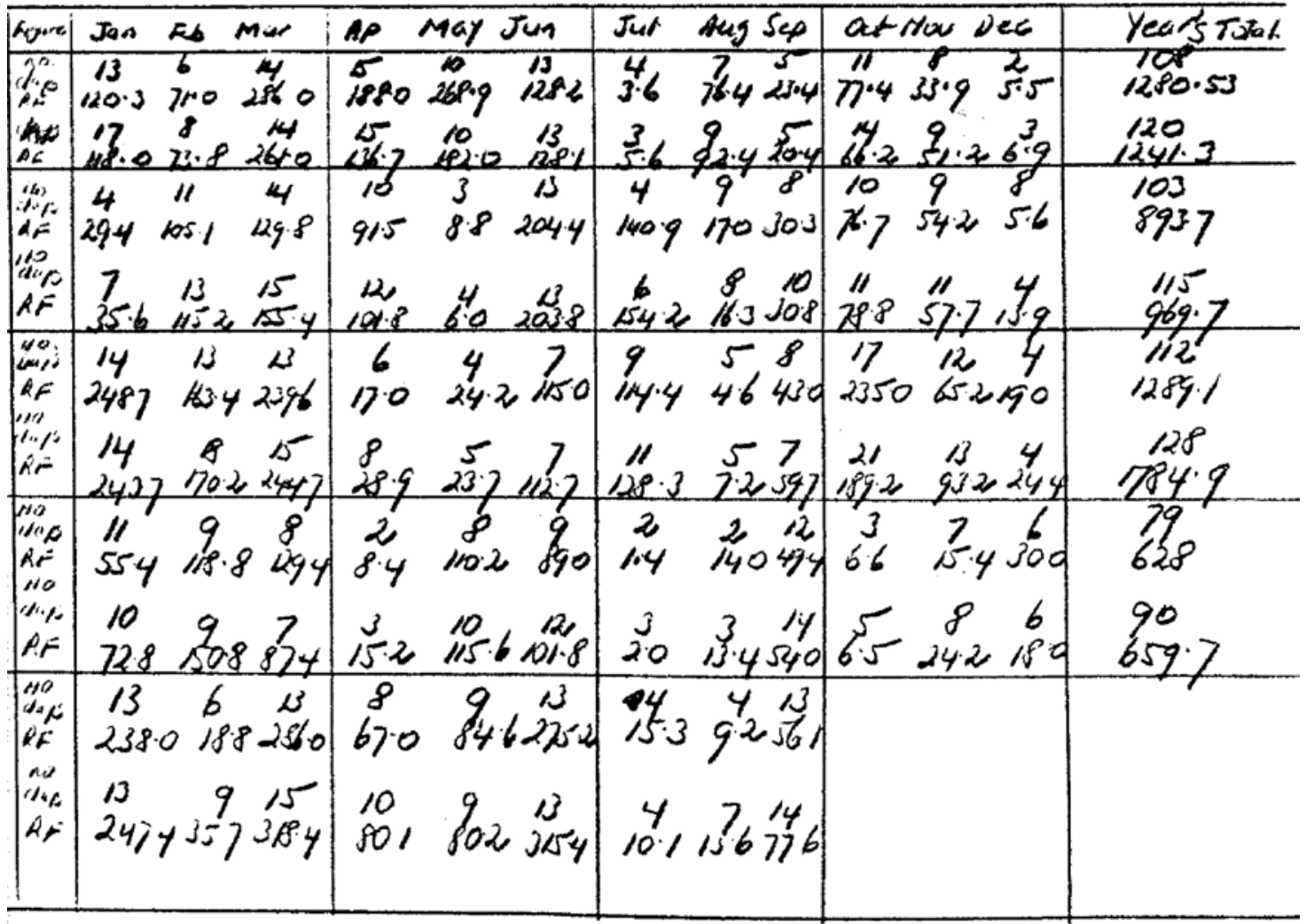


$-4-$

But I doubt that the water hyocinth (bichhornia crassipes) and parrotes feather (dvelophylluse prasilienge) in the mall, quite shallow pond in it the tip at Georges Avenue, lookwood, are anything out recent jetsam from an acquarian that must fall. The quiokiy maturint rushes and sedge-like plants (Junous cepitatus, J. continuoue, J. planifoliugs Scirepus prolifer, S. Inundatus, S. chlorostachyej that srow in and abo ut the edge of this and other sowewhat wore transient sinetrs of water seem better adapted to the alternately flooded and sun-baked and cracked soils that oocur in such areas. loodenia paniculata, a fleshy rooted native perennial is competitive in sowewhat s lillar situation. If the water is 108 persistent. Uther rughes, sedges and herbs frow well where bul ldozing, digsing, wheslruts and erosion cause the soll to be under water too long for most other plants to survive. Some are drought-reaistant perenniala, others quick-growing annuals that can mature and seed surprisingly quickly, cymerus tenellus being aolo to go througch this cyole on uneven paths and grasslands during a long apell of wet weather. The seads of other plante such as Senecio hispidulus and bigigeron floribundue can also be washed into these short-lived pools, germinate profusely after the water reoedes, and then survive with varying degrees of sucaess.

The edres of creeks and waturhales: other drainage 11weg

The Duok kirer has a profile that has been shaped by the great fluotuations in the volues of water it has to carry. The bed is deeply inolaed ten to twenty feet below the plain and 18 elther permanent pools, exposed claye and rock or shifting beds of allt and road gravel all confined between 10w inner banks. On one side or the other there 1s often a broad bench or long slope back to the outer rim of the creek and here moisture levels, humidity and perhaps nutrients tand to be at their highes $t$ particularly if the banks are wooded and shaded. Hut the flow of water oan be suffloiently violent to flatten shrubs and even undercut and topple trees particularly where the river's course has become unstaple due to the east bank of the golf course having been reclaimed. Wost roody plants, therefore, now perch abouthe rim of the upper oank though upstream a few survivors and sekeletons sugsest that trees and tell shrubs once down to the inner bank. Back on the platn the natural dratnage pattern has been truncated but not entirely replaced by culverts and stormwater ohannels. Thefo rty foot oontour on map IIX outl ines the course of four such gul lies, each of them oroad and U-shaped, rarely more than four to six feet deep, but still lonis enoush to carry up to eighteen inches of water in heavy storms and to reusin moist with seepage for some time after particularly where there is any shelter. Those on the west bank still flow into 1mpermanent waterholes on their lower reaches of the sort that probably formed the $s$ wining holes that once were found on Haslem's oreek before they were replaced by stormater channels and the waterworks of the larnarvon lolf course. 
$-5-$

These drainage lines seem to have been deliberately clearea of tall shrubs and trees particularly in the vewetery where the scruo tends to occur in reglmented stands well back from their edges. But the urickwark of the channels there often as not has blocked local surfice drainase water which has eroded new natural lines alongside them.

Wost of the plants collected can be found oxowing adout tilese watercourges as weil as in many other places. but others brow only on thein, beut for a varlety of reasons. Un the buck kiver Hakes suricea and perboonin linearis are riverside plants probady because only there are they muoh pr oteoted from the fires that have kilied them off on the plain. bich the same reason may explain why the only specimens of loodenia ovate, Asterolas 1 a corresfolia, Leucopogon lanceolatus, romaderitis lanikers. Pomaderris fermugines, and Hovea lonkifolia oocur on the banks of the river or the deep drainage easement on 1to west bank. ur it hay be that some of these species are there simply because birds cowing into drink have passed seeds which they have brought in from outside the sample

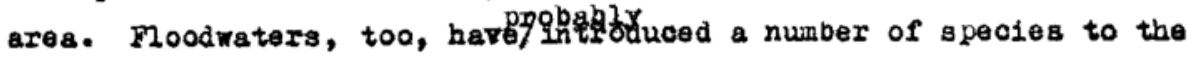
banks of the river such as the Arum Lily (Kantedeschla aethiopioa), the swamp lily (Crinum podunculatum), the hippeastrum (Hippeastrum $x$ equestre, Dutch hybrid (?), the snow flake (Leucotum aestivum) and the madéris vine (Anredera cordifolia). Uther speoles, particularly many of the exotios, grow on these sites because they appear to need the roister soils, higher husidity and enriched solls found there if they are to survive or to be competitive.

So agsressive are some of these exotios that they they tend to be dominants which suppress and exclude many natiye planta. "here soils are moistest on Irak Kiver oreepers such as Jasmine (Lonfcera faponica), the balloon vine (Cardiospermum grandiflorum) and occasionally byrsxijhyllum ssparagoices form layer on layer of runners so quiokly that fow herbs and small shrubs other then the reed (Phragmites australig), fennelfoeniculum vuisare), and retsonia (ilatsonia bulbifera)esoape tinrough this canopy whilst the larger shrubs and trees are weakened by it, particularly by the fires that the creepers carry up into their orowns. Where shade is dense the wandering jew (Tradescentie albiflora) also forms dense nats. A little h1gher up the banks prairie grass (Ceratochloa unioloide日) can de equally aforessive in sesson as can kikuyu (rennisetum clendestinum) wherever it spills over the banks fron playlut flelds. A sowewhat different range of exotice tends to we dominant on cleared drainage lines that are only seasonally moist, there usuaily being a paspaiuff-couch (Yaspalum dilatatum-iynodon dactylon) association of seinly exotio grasses and herbs on such sites. Ihe t wo dowinants in this assodation show a wide range of tolexance, fift ther being competitive on soils that are continuously molst, both bein capsole of ploneering quiee dry exposed sites particularly where the soil has oeen distrued, but each seeming to be at its most vigorous where the soils are quite noist for much of its growing season. However, the two more inportant herbs 


\begin{abstract}
$-6-$
that form a substratue in this agsociation on the buok kiver

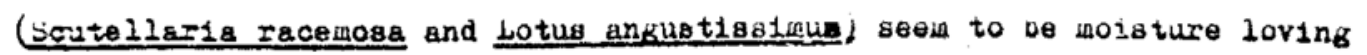

s.ectes that survive dry seasons oither as seeds or as a fleshy root

syscem. in kookwood cemetery this as8oolation (but without skulloay, apjerentlyj 19 often replaoed oy 8 tands ot Hatsonie tulpif'era so dense

that they exclude most other plants. But th1s species also falls where soils exe under water for any length of tine and graduelly thing out upalope to occasional scattered plants.

dpar $t$ from the cominon reed (fhrataites australig), which is found anly witin the osnks of the thack Kiver, most of the nutive species

that prefer xoist soila seem tolerant of a ranje of havitawa. Lven quite soft, moisture loving herbs such as the pale knotweed (yolyganum decijens) and the common ranunoulus (Kanunculus lappaceus) oan iror 1. places that are simply damp and sheltered whilet waterouttons (Cotiul a coxonopifolia) can brow If the solls are simyly seasonally damp. Shribs and trees such as Caguaring glauca, Callistenon salignus, Leleleuce ingarifolia and Leptospermum flavescens seem tolerant of sites near permanent or seasonalwater, damp sites, or even slopes in the caseor the swamp oak. Tree species that obviously brow and reproduce best wiore there is permanent water (Angophora floriounda on Luck kiver) $\propto$ about inpermanent waterholes (the fine stand of jucalyotua emplifolys apout the oully at wellington road seem even more adapatable and aole to extend out onto the undulating ploin (New street and St. Johns hoad for the red oum, Kibo keserve and KIngsland koed for the angophora). Giren the zange of habitats towhich all these species can adapt probably noze forsed dominant atands on the permament and seasonulwatercourses but were simply more common in the original woodlands at these places. The stends of swamp oak in the cemetery and those of Ancouhora fluribunda and the red gum on Duck Kiver seem recent and are probuoly due to distiurpance.
\end{abstract}

\title{
(b) lxpoged soils and subsoils.
}

Cool orsss and scrub fires are constantly exposing thetopsoil out in most seesons it is quicily proteoted from damage by regeneration from roots, stems and seeds. Where it is not orftefere gubsoil is brought to the surface oy erosion or interference the habitat becomes a far more difficult one for plants. Soils thet becone comploted, baked hard by the sun, and poilshed of the wind and the ruin offer few rootholds other than the fractures that occur in these vertically struotured soils. juvsoils are even more difficult for plants to colonise, particulariy the guosoil a on the Duck Kiver sandstone s. 'hat rock oreaks down into a ilisely divided clay that is euficientiy unstaole on slopes for tire surface run-off and perhaps oven the impactos raindrops to underaine seedlings 
$-7$

or to orerwhelm them with debris. The red claya are also easily eroded. in such sites each ploneering plant must result from a delloate balance that allows for the chance lodiement of a seed on pere and often sjoping fround, 1ts nurture by rains suliticient to keep it raolst yet not that heavy thatthey undermine or awanp the seedling, and snelter tist neither suppresses it nor allows a few days of brifht sunshine to iessioete the seedbed.

Yet plants can adapt to these exaoting requirements and the tougher and acre prolifio among them can outld up into quite large populations. If there is a source of seed even seedings of tree species such as

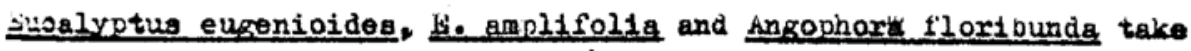
on the bared subsoll of the breakintays in the clay pits and bullies on Duck jtrer, 978 having been a particularly good year for the germination of ingohores. Howeverx, shrub species are more usual with those species with soed that has adaptations that ensure it isspread wide perhaps being the first to co lonise such areas. Cassinia sxcuata, which has wind 0 lom seed, is often the most commonand tallest shrub and it may be thet once established it attracts bixds which bring in seed of the tougher wattlea (Acaota faloata, Ao longifol18, A. bromit, A. pubescena) and pea-flowered ilants (Pultenges villoga being the most common though

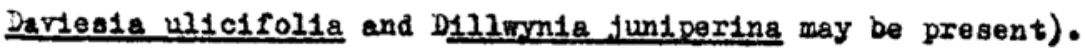
Seattered tuftand tussocks of tough native and exotio irasses are also well-represented (Danthonio purpurasceng, intolsgla stricta,

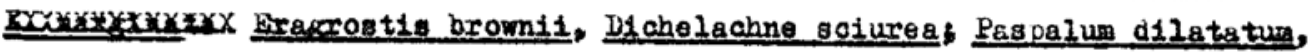
Gyodion dactylon), possbily because their seed is windolom or spread

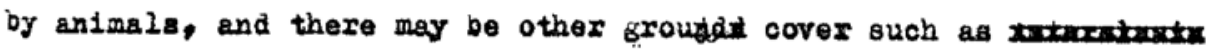
istoloma hums fusum and Hardenbergia yiolsoes. But Kunzea ambjoug, though often wltimately dominant on auch solls, maybo absent if there are no fruting speoimens nearby as the seed seems spread mainly by windrthrow orwater. Hakea sericea is an equally proflfio species, if present, though how so large-seeded a species the seedlings of which are rarely found more than a fer maxryards from their parents came to be 80 Mdespread in the diatriot 18 a n10e question. Indeed, many of the native species that grow in these exposed sites are so light-demanding and their seedings so intolerant of overmolst and shady conditions that they may once have been chancesurvitors in the orifinal woodland. sut nowadays it is the very clearing and ourning that once nay have cause $d$ an explogive increase in their numbers and spread than wide that threaten their very exiletence.

io doubt in the furiness of tiwe the soils on these disturbed areas onoe reverted to an eucalypt woodland. Joday that suocession need not ocour but may be cheoked by fire, further clearing, or be the interposition of a new and phrhaps more stable phase, that of grasslande which include a high proportion of exotio species. Un parts of the clay pits on the east bank of Duok river a form of the paspaium couch and herb association and a mixed pasture of those and native grasses suah as 
$-8-$

Dichelacine soiureg and trakroatio orownit way well estaolish if the area is ever kept free of minl oskes. However, wherever casoinia arcuata SOWB well in these pits and on parts of nearby sewerage and drainace esecents it and Its associater, fultenaes v12100e and the wattles, seem to be able to keep the exases sufftciently in check for surther shrivo seedlings to take. It may be that these areas, once the soil profile becoines more mature, could in turn be invaded by Nelaleucas or even eucalypts and ultimately becone woodlands. But wherever the zenes of Kunzes ameigus are present the sites with disturbed s01l profiles rould seen first to become Kunzes sorub, which is the typioal regetation type on much of the southern slope of the crematoriun Hill and the rim of a sinall clay pit on Duck kiver at frinces koad.

Anzes empigus with its prolific regeneration, its need ror intenge light, iss spreaaling habit of growth and dense canopy, 1ts height, and the large asount of litter it sheds, quickly becopes dominant in these scrubs. Other taller shrubs such as the wattles, occesional melaleucas, particularly Kelaleuca nodose, Hakes serices and lasuarina littoralis $800 \mathrm{~m}$ able to remein part of the community by their natural inorease in heigit out are able to reproduoe only where the eround is more open. vall shrubs such as Cassinta arcuata, Futtenaea villosa, Daviegia ulicifol is

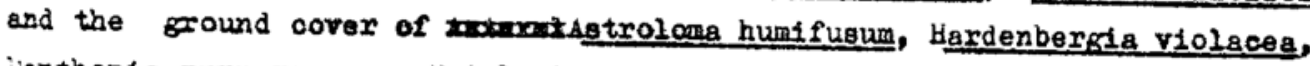
jenthoris purparescens, jintolasia gtricta and so on at best linger an vefore Fadually being suppressed unlessthey are able to colonise the edises of the scrub. Tree species are rare in theexisting scrubs, but are so everjkiere in the Cemetery and on the cllotrent on buck iftver where tho frepents of it occur. Seedlings of angophore floribunde do occur ciceak of jowl with the Kunzea at prinoes road and eucalypt seedings cen estaplish on the soils of breakikways out I have not seen them do so in direct competition with the scrub. Hovever, the Kunzea, wettie日 and the like are relatively short-lived and the ecrub would gradually ve opened up by deaths, wind throw and fires to the invesion of other specles, particularl y once the soil proftlle became more nature. jeeding eucalypts misht only establish by chance, of course, out most seem to do that answay. Ultimately they would tend to dominate and suppress the scrub and it would revert to eucalypt woodland in tach the saine way as aome of the titiee scrubs are now doing.

\section{(c) The j lain and Undulating Land}

bere the micro-environment seems dependent on and difitirs in each vegetation type. And the structre and composition of the vegetation, within the range of possidilities nature allows, is deteriained nowadays very mach oy man and fire.

At rookrood 'the bugh' and the 'burial grounds' have always been Ircompatiole and 'the bush' also irreconoilkable with the Victorians' concept of'a necropolis' as a beautiful tarden of lawns, shruboerios, 
LARGE HOLDINGS

CHISOLM ESTATE

DUCK RIVER

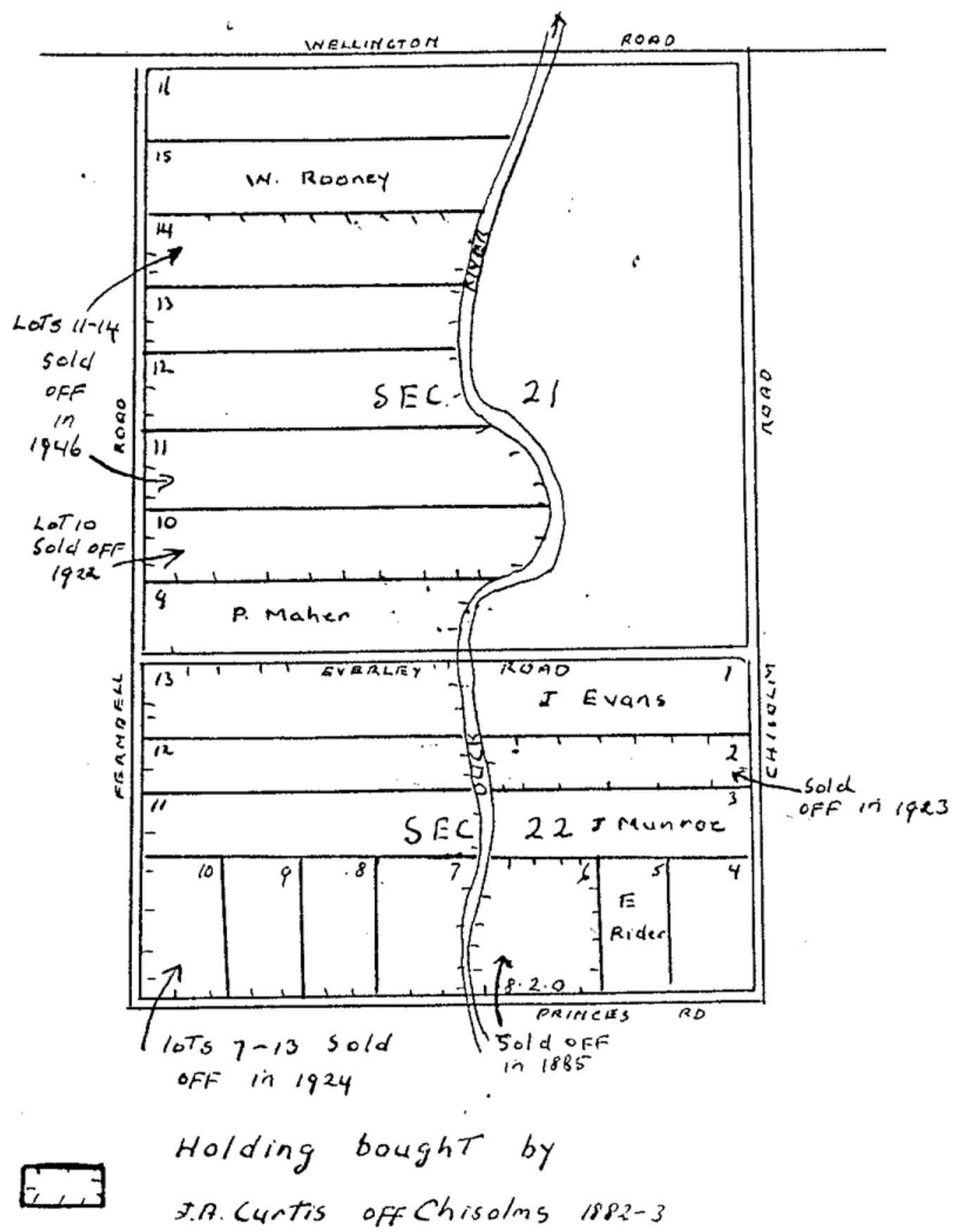


avenues of trees, ponds and fountains. Yet the usihland survived as manageable themeda grasslands and depauperate scruos alone swaipy drainase lines and fullies and out of sight over the watershed towards lhuliara. It did so and now even encrogches onto the older vurial brounds only decause of a latterduy preferenoe for cremation that has leit some waste land and inpoverished the various denominational trusts but fire, until recently, decame the trusts' one economio tool of sianafement and grassiands, scrubs, shrubberies and avenues have veen indiscrimminately burned for many years. Grasslands and scrubs survived, estaolianso on uneasy equilibrium with one another, and seebed likely to become permanent vegetation types until grave-dig8ing became mechanised and bulldozers were able to muke short shrift of both. The old itate fospltal ground (which once included the Carnarvon Golf lourse) have been even more completely cleared of all but a few veteran trees and shruba, perhaps because 'The OId Men's Home' was expeoted to be self-sufficient in vegetables, milk and cheese. However, it may well be that these areas and the cewoteryz were at least partly cleared for grazing much earlier as they were all fa part of the 1350 acres of the 2640 granted richard Hyde lotts which were topused by the crom ing (about 1860?).

in Duck Hiver the relation between the present vegetation types and the past use of the land is much clearex. On Map III grasslands give way abruptly to gum and ti-tree sorub at the old property boundaries, the one atand of tall woodland that has re-established over some seventy years 18 on an unasable part of the Hillston market garden, and the one other area of low woodland 18 on an 180lated part of a property on the vest bank. Once the whole of the survey area was part of a 600

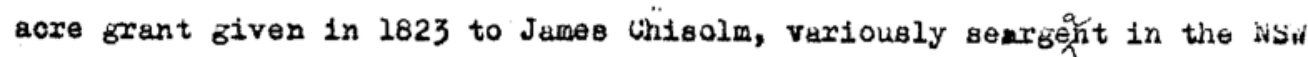
corps, proprietor of the Thistle inn in leorge street, businessman and then grazier. The grant ultinately became part of a larer estate, 'bverley', named after 'hisolm's property at kedfern, but whether or not 1t was much cleared and worked in with the lhisolm family's other properties at Narelian, Goulburn und the bland I can't say. But increasingly it became more profitable to subdivide and sell the land though, beins low lying and near the river, it was surveyed into faralets (see jiegran I) and not into suburoan allotimentsy of the sort that seemed likely for a time to turn the ridge towards inuurn into another midale class Arcadia lik e Strathíield. In 1882-3 all out eight of the twenty-one allotments into which the survey area was suodivided were sold to J.i. curtis who, perhaps sibnifioantly, was a jydrey timbar merchant and he, in turn, sold all out $8_{i}$ acres to Kovort lhadwick in 1885. The various members of the chadwick fuwily (some of whome are sonetines described in the transfyredocuwents as 'surveyars') hald this property intaot until 1922-4 when the southern allothents were yold uut It was not unt1l 1946 that they perted with the area which now includes the best preserved areas of bushland. That oush, I would cuess, 3irvived 80 long sipply because it was on tilis large and possibly speculative 
holding. The market ferdeners, dairymen ana the like usually could 1ll-aftorc to let their land lie idle.

If the exact chronology of all thits atstruotion is in uoviti its outcome 1s not. No stand of the orfisinal vegetation aurvived not even on the Hillston property where the fine oroup of euoulypta has regineratad slace 1902 from a cleared but badly placed chlcicen tiaris. brost of the orlifinal species no doubt atill oocur as cleartne with axe and lire withoul years of lavorious gruboing and weeding la soinewhat akin to sowing dragona' $t$ eeth. The native species able to regenerate from footstociks, lignotuders and stumps range through the full gaidit of growth forms from forns, soft herbs, areepers and shrubs to all the dowinant trees. wing of these and other species also seed profusely and would have found a cleared and ourned seebed ldeal for their regeneration. Froof of the native species' toush, endurinis qualities lie both in the proportion they atill form of the species list and the way in which they still exclude all but a few exotics froc nost stands of sefenerated sorub. What was destroyed, and that probabiy quite quickly and perhape iredesulaoly, was the struoture and composition of the orifinal vegetation. for what was once probably an area of tall woodland or dry schlerophyll t'oreat

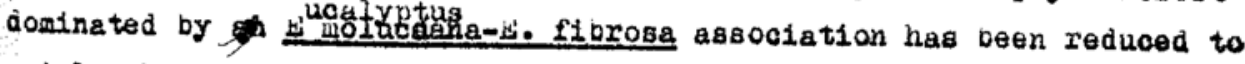
mainly the erselande and depauperate eucalypt and melaleuce sorubs shown on the Maffi. Both are unstable, the Brasslands being prone to invas10 $n$ by shrubs and trees, the sorub having a propensity to revert into woodlend. but firea are frequent, as often as once in two years if sufficient cead litter accumulates about the base of grass tussocks to fuel che the cool grass that are most comon. host woody plants lack the chance to rtestablish and raise their crouns above the flemes before they are burned baok once mosell. Those that are tall enough to be unaffected rarely have the girth to be free of the risk of beints axed back to fire aensitive regeneration from stumps. Systematic clearing may have first formed the grasslands and scrubs but nowadays vandalism seems auficient to keep them indefinitely as the dominant vegetation typ es in most places.

\section{(1) The Grasslands}

1. Grasslands in which alwost pure atands of ghemeda australis are vominant occur where the land has been cleared a lone time and little interiered with other then by firing. In the veinetery the largest ureas are in the unused parts of the Indepandentand wethodist burial irrounde vut the siecies has also re-estaulished its douinance on trie/stopes of the Fresybyterian vurial ground no. 2 despite the masive disturbance there when it was in use between 2890 ard 1910. Un juck tifver the one large atand of Themada anstralis to back a little froin neliline tor houd and is more an open, low parkland with a scuttered upperitorey of eucalypts and ti-tree. Hisewhere on the river fiost colonies of the trass are small and in open glades in the scrubs and woodlands. The two variants 
$-11-$

of the glecieg $\leq t$ rookwood form discrete though adjeinine poililatione, the one a muci zore robust, uroader-leaved and more glaucous ford, the other a finer, $G r \in \in E=$ ard sinaller one which is the only form t'ound on luck kiver. both are viscrous perennials that flower and seed jrolificeliy in early sumwer unlesa jurzed in eprine, a dry season seemire only to affect the height of $=5$ ians. Tussocks are cloyely spaced, leaves arch over between the $= \pm 0$ alzost touch, root systems are flibrous and agcressive so that there is $2 i t=1$ e light or gurface woisture in an undistrubed stand for the seedifrs of competine species or, indeed, for those of lhemeda. Yet other s jecies are often ussociated with the atands, launy of tham

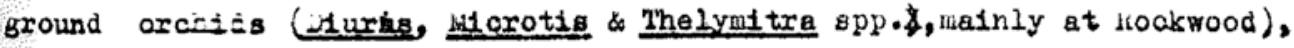

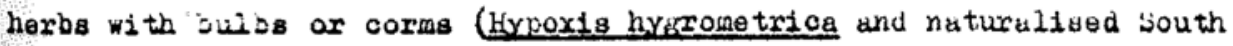
African plants suah as homules longitiolia, ireegia refracte, and Ixia, Watsonia and Fitonia spp., the latter three only at kookwood), patersonia longifolla (200<wood), Xanthorrhoeg and Lomandra spp.; and a l'ev low shrubs tiat can survive if fires are not too frequent to stop them regenerating $f=0$ rotstooks (Lisanthe stricosa, chorizena purviflorum, Bossaea buxtiolis, p latysace ericojdes, last species kookwood onlyj.

These grassiands persist only because they are often burned. Yet fire, whon followed by wet ther probabiy holys to estolish the seedings of shrubs and trees micis, were they able tosurvive and to build up into large popplations, wowid ultimately supress the light-dewanding ghemeda australig. But in the cererezy, so far, the $b$ alance seems to favour the erasses. seedlings of soze of the old avenueplantings such as pinue radiata, and Eucalyotio seitigna do establish but seem to have betn no more avle then the incre somion of the native eucalypts (bucalyptus resinifers and

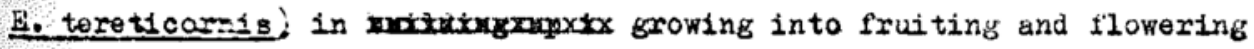
specinens as cistinct from transient populations of saplings. anxophora bakerf with its rough, more fire-resistant bark and its eurly flowering and frultng haoit might be more successful were it more widepread vut, for whatever reason, it is not. Leptospermum ettenuatum therefore is the one shrub species that has built up large populations of mature specimens but these are peinly in the burial grounds and in any case seem naturally to be scattered and to form crowns sufficientiy open to ve tolerant of the grass. kelsleuca nodosa frobably poses the frestest poxtential threat to icese srasslands beine a sjecies that is able to re-senerate on a wide renge of sites, one that is fast erowing, quiokto flower and well able to ouild up a population of lienotubers that regenerato after fire and provice an environment suited to the estaolishnent of other shrub species inkich far more shade-tolerant frasses than l'hamea are soonsupressed. But fires in the ceinetery keepthe stands to neleth barely more tien one or two raetres hikin and provavly help corifine them to the moister socikage lines. Some of the wore cownon asscciates of ielaleuca nodosa in these suall scruos ace themolves less of a threat to the grasslanis as they are mare fire-sensitive, tris tioush tive jinrote aoacias (Acacia parramattenglg, Andeslogka, h. pupgscens) olicker from the old root syatem after fire and the hesth-like pea fluwered sjecies such 
Pultenee yilloga and $P$. miorophylla re-generato profusely from seod. Yet they survive in the scrubs and in more sheltered places avout the

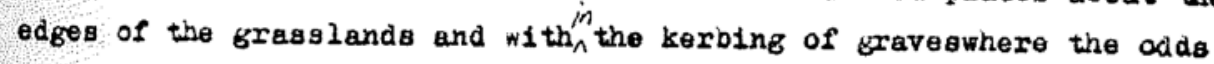
arainst their being burned are higher and could increase quite rapldily In numoers if ftres were less frequent.

In the open pariclands on Duak Hiver the situation is more c amplex as the eucelypts (Eucalyptus molueanna, bi flbrosa 8sp. fivorose, bi ougenioldes E. longtejia, 3. resinifera) and the ti-tree (Helaleuca decora) must once bave deen able to regenerate successfully for then to form an upper storey to the stand of Themede eustralis. However, fer gum or t1-tree sedinge appear to have established for quite jome time and the stand comes close to having an even-aged appearance and may be the firat phases of reganeration after these allotments pere cleard when exasses may or may not have been as couruon as thoy are now. Lost elcalypts are roughly fifteen to twenty-five feet high unless they have been axed or cut baokby flre. The t1-treels also old most of the plant being from perhaps elght to fifteen feet iligh though the popuiation of smellex stems with diameters from 3-5am is of indeterminate age as such fire-sensitive specimens can regenerste many tines when demaged. The laok of young eucalypts is partly due to the absence of many mature, fruting specimens but even where these are found there are no seedifiga and fel young plants. The ti-tree, though also slow to mature, does nor fower feely and set seed and it may be there are no seedings because the quite exacting conditionsthey noed they are to astavisis are not being t. Young plants of Holalouca decora are rare evezpinere in the diatriot but the one congested, even-aged stand of several hundred specimens I have come aoross is on a site at kookwood so seasonally moist that I wouldguess regeneration might bo abundant in a period of quite wet weather on a suitable seed bed. But the most favoura ble seedbeds in these perklands are within the occasional 8mall seruib which already have a fairly full complement of shrubs. Here the c.nopy 18 closed and casts aufficlent shade to exclude all Brasses other then scattered tufts of shadetolerent species such as kintolsis Earginats, B. atriota and Microlsena stipoides and there $1 \mathrm{~B}$ much bare oround. Onoe the canopy is opened up by fire, the accumulated s tore of acd is better able to orminate on ground that has a muloh of scorched and partly ourned ti-tree and gum leaves and the seedlings are the o otter able to surrive as there is broken shade and no establigh root system near the surface. The nearby gxasslande, when burned, are often deficient In seed, the ground tends to bare and unshaded at first, and such plants that do estaoligh can be quickly overwhelmed by the lea ves of the regenerating tussocks and deprived of moleture by their estaolished, quite ageressive root systems. Clearly there ls no one, sinple explanation why the gllanas has been tilted in favour of the $\mathrm{g}$ rasses In these perklands. But here, an at kookwood, they form a ralcro-environment favourable to flre and, being trpublio places near residential areas 
$-13-$

areas, are jrobably being burned so often that the tree and shrub apeciea that otherwise would supplant thegrasses are now unable to re-estaolish their dominance.

11. No onespecies of grass is domfant on olesred lands where the ortginal grasses have been much disturbed by grazing, clearing, levelifing and so on. Usually the mix 1s of native opecies (1)chelachne gieures,

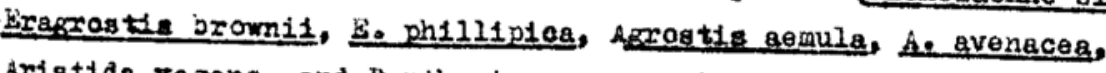
Aristida rasans, and Danthonter spp particularly Danthonia purparescens with a larger proportion of exotios, particularly of the specios that axe dorainant on the cleared, a easonally wet drainage lines (paspelum d1 atatum, Cynodon dactyion, Briga ninor, Setaria geniculata). Themeda alistralig, if it occurs, tends to forto sinall isolated colonies or solitary tussocks as it is killed off by over-grazing and does not ealily re-establish on distmbed soils. The density of the exard varies with the season a nd the coloture atatus of the sita and say be yexy close on drainage lines even in dry seasone but quitesparse and open in exposed places. Usually there are herbs, some of them auch as Centaurium tenuiflorum, and C.arythraea, ord Linum tromom trischus and sybepetes orontium tolerant of a wide range of sites, others sach as Lotus angustisg1raus, and scutellaris racomosa andso on forming a substorey to the grasses on molstersoils. A vari ety of emergents such as the dooix (Rumox exispug), Verbena bonariengla, thistles (Sonohus spp. C1resum vulgere) and rusher (Juncus spp.) also occux. Grasses such as Vulph bromaides, V. Byuxos and Sporolobus africanus near tracks and kikuyu (rennisetum clandestinum) near dumped spoil can build up quite siseablo local populations or even, in the as e of kikuru, extude most competitors.

There seess to de an uneasy balanoe between the species in these stands. ifferences in their growth habit and their seasonal flush of flowering no doubt kelp the low annual, Briza minor, and the skindider native perennial, jichelachne sciures, butld up such large populations In a pasture which in winter often oeems to be alnost entirely coarse, leafy tussocks of paspalum. But their quick response to the first warm spring rains would probably be of littie avail in the 1 ons run were the more azgressive but moxex waxmth demanding paspalun not checked from becoming rank and choking out most of 1 t competitors by repeated burning. The composition of the gxassland also changes with the moisture status of the siteand might therefore also vary over a oycle of wet or dry years. The molstest sites are very much the preserve of the exotic is rasises and herbs with anly Afrostis gpp. among the native grasses being at all common thoughboth Dichelachne solurea and Mcrodsena atipoides may be present. But native species such as Dichelachne sciurea, brasrogtis orownif and urletida vagang are auch more successful on dry sites and are ableto bloom and set seed protilifically in a dry season such as that of $1978 / 9$ when the paspalumand couch remain stunted and scarcely able to renew their crowns lot alone form culing. In such 


$$
-14-
$$

area s, Inqeed, these grasslands may be susceptiole to invasion by Thomeda australis. For that crais soems to ve re-estawlishing on the dry ex ased soils along the southern boundary and alone the tracks throuzh the old dairy on Viellington Road at buck kiver. ind if such an invesion is possible during the recent cycle of wet years it 1a conceirable that in the past the grass could also re-establish its dominarce on the well-drained slopes of the older burisl crounds in the
Cemetery. HOst of these areas seem to have veen d laturbed too recently for there to be much evidence of their having been invaded by shrubs or trees. However, about bverley kaad on the east bank of buck kiver a population of wattles (Acacie falcata, A. longlfolfa, A parmattansig) and peaflowered species (Pultenses villosg, leline nonspessulana) have outlt up among the grasses on dumped heaps of spoll. The sate species and others from the rim of bushland along the river (bucalyptus noluccana,

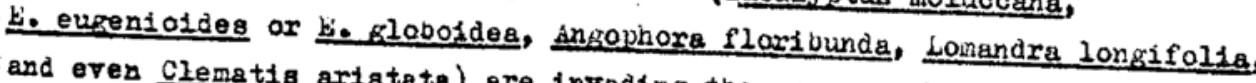
and even (lemstis ariatata) are invading the grasses that have taken over the western end of the old Hillston market garden. However, both areas have been freefrom fire for at least three years, probably more. And though they suggest that grassland ia culy a transient vegetation type In this district the succession to woodland must surely be a very slow one as even the Hillston property, solated though it is by the creek and road, has nonethelese suffered a quite violent fire in recent years that has killed or seriously weakned a number of large eucalypts, cut back the resenerating trees, and led to the formation of 1 a falrly mature second
seneration of wattles and pea-flowered species.

\section{(2) Sorues and low woodiands}

Ti-tree and eucalypt sorub (or variants on it in which such species as injophorg baicer1 and Syncurpla Blomulifere are also dominants) is the most cormon typ of vegetation on land that has veen partly oleared or which has been recolonised by shrubs and trees. In places on the vest bank of Duck Hiver the eucalypts have becomo dominest and are tall
enough to form a low woodland.

A t their simplest the eorubs are dense stends dominatedby one or anotiher of the three more important $t 1$-trees, Melaleuca decora, M. nodosa and 14. styphelloides. Scrubs in which thedominant stibey is almost solely of Melalouca nodosa are not es common as might be expected of this agressive and tolerant species, the only one that is visibly and actively colonising new Ground at present, but they do occur in kookwood and as steall stands on the Luck Kiver. However, they are rarely taller than fire-sensitive nine to fifteen feet and, as the stems and crowns are quite to ground level. Helaleucs otyphelo of being cut pack by the flames, of ten to ground level. Helaleuca styphellosdes is also fire sensitive 
$-15-$

and an 1rdifferent coloniser (and therefore, pexhaps, has veen ellainatod from kookxood wich is rarely a dominant shrub. but whan freed froin fire,

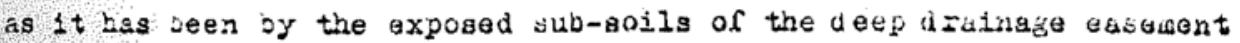
on the aest bark of juck liver, it can form tall scrubs which so bat are up

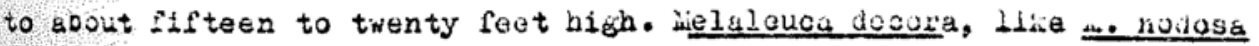

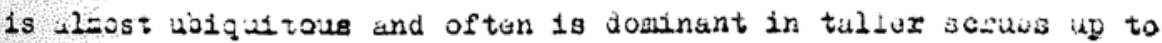
flfteen to twenty feet high. Height for height tt seems to ve able to budld us stea with a frouter diameter and perhaps a greater tricknesa

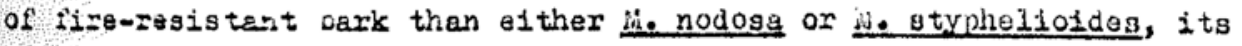
crown seess to heve a distinctly bettor abilicy to re-estaolish 10 gónched and, cespite its thick-stemmed, umbrageous habit in the open, can for slencier stems and nursom crowns in dense stands that anable it to fow up into the canopy even of woodlands incoinpetition with other syecies, perticularly the euoalypts. Wore usually the soruos ara not monosjecific stands of a dowinant ti-tree out inixes of several of the species rentioned and the saplings of some of the rough-barked und fiorous barked trees that are nost conveniently descrided as veine

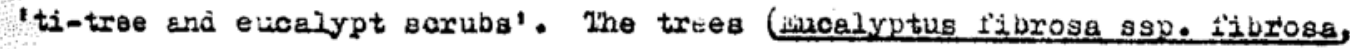
Eresinifera, Asophora bekeri, Syncarpia glomuliferg, the latter two in

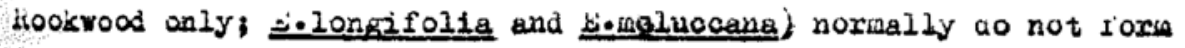
fairly pure stanis, the gre exception being that in the rrespyterian cometery dominantej uy 2 zophorg bakert, a species which has reproduced so freely from seto and sucikered so aggressively from the base after fires as to have iorzed a low scruid four to eight feet high. bilstwere, the tree species isve Grow torms and a frequency that range froil low, rire-genaitive frowth from occasional lignotubers that nay be part of the groundstorey in a scrub, inrougin secimers that axe co-dominants with helaleuca decora in telier scruos, to stands on the west bank of buck wiver up to thirty fiee to forty feet inigh that are becoming douninants in their own right and formiro tither sassy low woodlands, usually where hielaleuca decors is an imortant elezent in the stund, or low shrubby woodluids where it ia not.

The scrios owe tineir form and their very existence to the ti-trees. Ine Innate visour, adaptability and persistence of the three dain species have ansoled tiven to profit most from the vlearing of the orivinal woodlands so that rowadkys they are almost uolquitous, dominant in wost stakes of the succession, and the species to whose ecolory others, even the eucalypts, have to adapt if they are to survive. However, when young the ti-trets are rarely s o dense that they are able to exclude or suppress otber plants and usually there is a wide ranizo of low shiubs, herbs and srasses present. Wiry panic (intolasia stricta) is the grags fiost able to adapt to thet out the shade toitrunt neadow

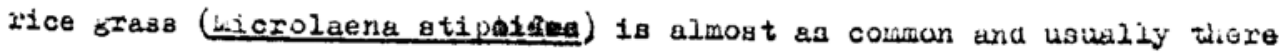
way be geciwens oi most of the native grasses collected present. Scattered herus of open kxassy places and the woodiands wily also cocur -

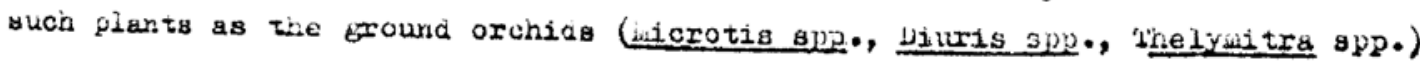


$-16-$

and the trigger flower (Stylidiun uramintfollum) at rookwood, varlous Lomandra spp. Dianella sppo, axd daisios (Venocio higejiciliag, Vernonia cinerea,

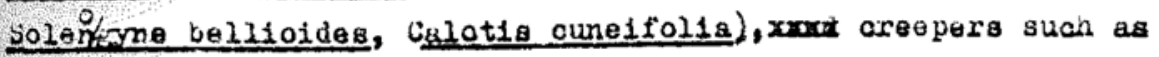
Glycine s:D. and Lyrgtphyllum asparagoides andso on. jhrubs of open places such es the wattles, various pea-flowered species (rulteiraes upp., Daviesia ullat folia, Dillwynis funiperina, Indikofers australia), preales inifolis, daisies (Olearia microphyla, Helichrysun disomifoltum), black thorn (Bursaria gpinosa), bottlebrushes (Callisteinon lineario, C. (Splnifolius, even C. salignus) and the low erowing tololoucas

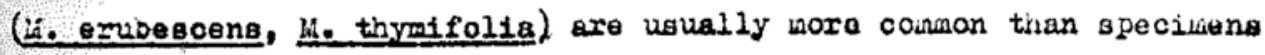
of species that prefer moister or more shady habitata suoh as rolyseias sambuatfolius, plttosporum undulatum and Breynia oblapgifulis. sut once the ti-tree beoomes fire-free it any forin a dense stand of even-med stems, even if originally the ecrub ata a pieceneal astrestation of many eenera tians of seedlings about a core of fruiting speciwens and not the outcose of ane profuse germination in an unasually yood seasan, and those stems may form a canopy so coriplete as to supress wost other plante ursble to match their rates of growth. It sone almost pure stands of Lele lever nodose on Duck kiver, for example, the canopy is rarely more than $81 \times$ to ten foethigh and fow plants other than tough herbs suab as Lienella caerulea, oud Lomandra spp. and a few tufts of tolerunt brasses oen linger on in the dense shade and the eccuralation of litter. Low stands of kelaleuea decora and hi. styuhelloides can be equally intolerant $p$ Eround lants and low shrubs. But most scrubs are nore diverse and more structured, the dense canopy of an upper storey of L. decora tending to be orocen up by eucalypts and the teller wattles so there may be sufificient light for there to be a gecond storey of a f ew of the nore fire-sensitive

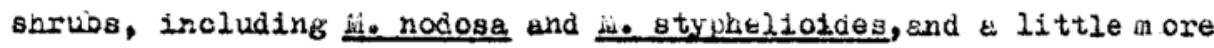
Erouna cover.

H1re, to the extent that it helpg keep the ti-tree in chack, does much to diversify and restructure the scrubs. Even the denesest of tham may burn and have their canopies out back, by a cool praso or litter fire if axly about eight feet high or if there is a secondary storey of shrubs, by a orown fire if taller, and once the floor la opened up to light it forms a very favourable seodbed that is susc eptiblo to invasion by other plants. Often all that results is an opheseral though flourishing ground cover of fire woods from setd which has been washed alown to the site or brought there by birds. Un Juck kiver, where the store of suoh seed seems somewhat exeater than that in bost parts of kookwood, the most oomen plants to apyear are short-ified

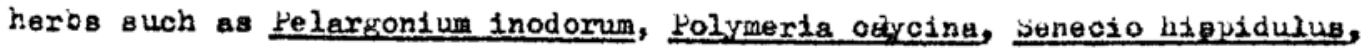
jolenue spp. and occasional thytolacce octandre but shrub upecies may slso establigh, Cassinia arouata, Helichrysum digomifaliuas, Ulearla microphylla, ilmolia linifolia, Hultenaes villoga, hcacla falcata, and Acacia longlfolla being alaung the wore common ones. 
Uccastonally these seedilines may come up so profusely about the edges of a scrub ss to choice the site and are oradually thinned out by lack of moisture and ligat as they compete with one another and with tha regenexatarz basal suckers and crown shoots of the t1-tree. 3he stants, therefore, may be short-lived, out depending on when they esteolish and the seasons that follow some (usually the Cassinia, Hintlea, and some of the beliahrysua and 0learia) iney oloom in their flret year, others in their second or In following years, the wattles being the last to sature and often the only ones that are able to grow up into and diversify the cenopy. wore rarely, at least nowadays, eucalypt seedings can invade the sorub of t1-tree in much tire same way, there belng occaslonal tall a tands of Kelsleucs decors in Kookwood where young, quite slender stemned, wauk small cromed and very fire sensitive spocimens of sucalyotas resiniferg are flghting for a place in the canopy.

But fire can also simplify the scrubs if $1 t$ is too vialent or too frequent. On the west Dank of the Duck Kiver in the summer of 1975 (I would suess) a sorub fire killed off many of the emergins eucalypt dowinants with stems of 1 ess than $10 \mathrm{~cm}$. D.A.H. and reduced many others to lignatuderous regeneratIan that 18 now simply the equal of the original understorey of ti-tree. Populations of some of the fire-sensitive wattles and pea-tilowered gpoles also seem to be rebuilt only slowly ufter flres which in any case need to be at least leas frequent thon once every two yeara if the gene pool of oven the most prolific and quick-maturins spectes is not to be put at risk and exhausted. Neverthless, the interplay between fire and the natural growth habits of the ti-tree can also help o reate new opportunities for other species to recolonise even the densest, mono-specifio sorub. By constantly pruning of the lowest oranches, oy weeding out weaker opecimens, and byt stimulating the more vigorous ti-trees to grow up wards the ground is eradually opened up to lieht and in this dappled shade a dense groundetorey can gradually develop, one that Is probably the richer in species and broth forms of eucalypts are common in these tall shrubs and lov woodlends as their canopy is more open than that of the ti-trees.

Grasses ere the most common fround cover wherever vielaleuca decora Is an important constituent of the tall scrubs and low woodlanda, pernays becauseffire and because of the shade cast by the canopy, or perhaps decause of some past factor in the succession that eludes me. Lt 1ts siajiest, the sward is made up of species distributed in ways that correlate nicely with the moistness and the light otatus of the different sites. It the most exposed, sqasonally driest end of the spectrum in sistisy glades kangaroo erss (Themeda quatralis) may be dominant; in moister areas of

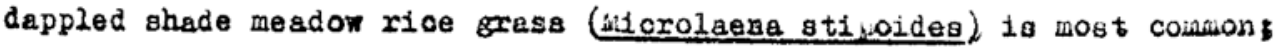
between the two extremes there tends to be a mixed stund with meadow rice grasa, margined panic (bintolagia marginata) and troxuctihodizeliog cressв8

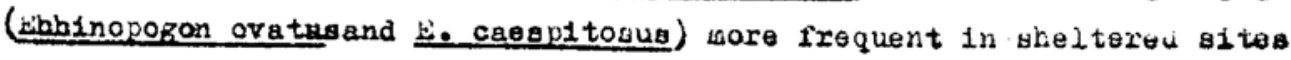
and wallaby orsses (Danthonis 8pp.) three awned syear frasues (mainly 
$-18-$

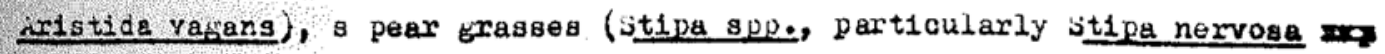
vare nervosa) and short haired plume g rass (Hichelachne steurea) and loves grasses (bragrostte bromil and hi. phillipica) mare comion in more exposed places. Sowe epectes show a wide range of tolerance along this opectrun, the wiry panio being one of the few grasses able to exor an exposed bare so1ls and yet able to tolerate the dense s hade about a substorey of ti-tree whilst short-haired plure trass is almost ublquitous. But many sites are too disturbed for these adaptations to hare sorted the erasses out. Themeda australis and itipe nervosas, for examp le, peraist as constituents of the sward in shady woodlands though they seer unable to rlower. Stands of Mlcroluena atipoides and intolesia marcinate censurvive in neat clrcles coout the sturaps of cut ti-tree in open flades or as larger stands in areas of felled and cleured scrub, Bomewhat thinned and weakened it is true and unaole to lower freely or grow well in dry seesons and therefore susceptivie to veling Invaded, of ten by exotics such as Pespalun dilatatun, Lotus angustissimus and Scutellaria raoemosa. More usualzy, the few herbs that occur in these swards are natives such a Diohondra repena, kalyweria calycina, Hardenbergia violaces, Brunontells australib, Viola betonicifolia,

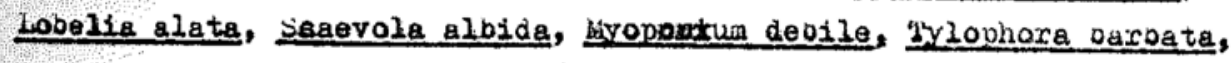
and even the maiden hair forn (Adiantum aethiopioum). But all are far fore conmon where the grasses are sparsest.

The understorey of shrubs and tall herben influwedudenels tolerant or moisture seeking apecios, many af them havinib seeds that are probably spread bybirds. The cheese tree (Gloohidian ferdinandi).

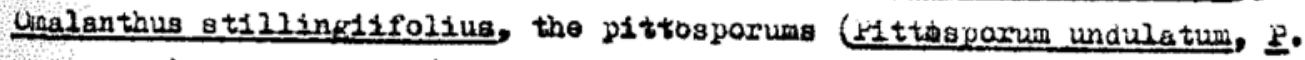
revolutua), the lentane (Lantana camara), the privots (cainly jicrestrum ainanse) are sowe of the less successful colonista yreynia oulonififolia,

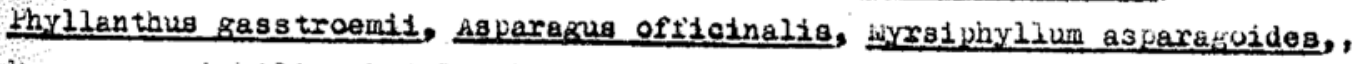
Kapanes yariajilis, Notolaealongtfolia with their ability to survive or even multifly after fires by suckering are soine of the nore successful ones. However, shrubs with a wide range of tolerance may estaulioh, many of them being fire weeds and plants of the sorups and exposed places such as the wattles, Hinelea linifolia. Helichrysum diegmifolium, Ulearia wicrop hylla, Lultenaen villosa, Daviegla ulicifolia, Indicofera austrelis,, the hop bush (Dodonaes triquetra) and oven accasional Cessinia arcusts. Usually such plants are sosttered specinens but in a fer places on buck kiver that appear to have been fire free some of them have ouilt up into a dense gubstratum of shruos beneath the eucalypta.

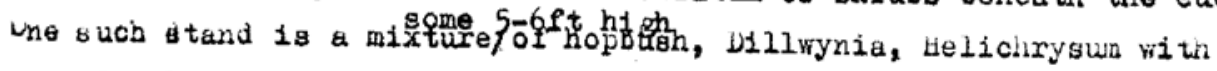
occasional bpacrids. Othere include small stands of species that elsewhere are only occasional plants in the soruts, the one population of

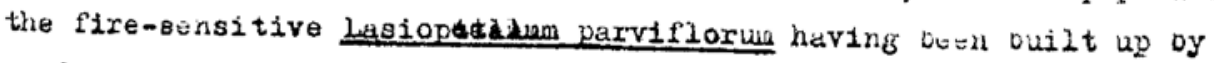
seecocarried oy flow of water, others of kulinizia pannosa apparentily Deline les ephemeral as the apecies both seeds frilifically ard suckers from 
$-19-$

1ts rootstocks after fire. However, fires saem to have oeen too frequent in nost places for a continuous low stratum of shruos to form. brodaoly constant ourning has also fraduilly reduced the remnants of an older, tall shrub and low tree stratum to laolated specinens, the currajons (arachyohiton populieum), forest oak (Casuarina toruloa), plack she-osic (Gaguerina littorglis) and the dogwood (Jacksonia scoparia) ouing on tho

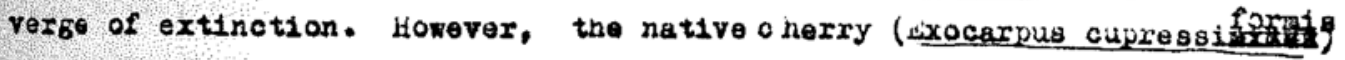
regenerates vell though there are only a few mature specicens.

The corposition of the dominants in the woodiands and scrubs has elso been altered, probably mainly by $f i r \theta$, and in ways other than the the o orious olas towards helaleuoas at the expense of the trees. The two dorifant species in the original grey box-broad leavec iranbark (buealyotur molucoana-ji. Pibrosa) assookdtion are also the last to casture Into fruiting specimens and are, at some stages in their growth, among the most fire sensitive speoimeno. Thus a single $f$ ir a eitrer killed

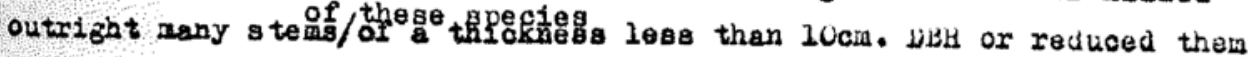
to 119 gnotubers whilst most of the red wahogeny (E. resinifera) and the voollyoutt (b. longlfolia) aurvived, the c rowns of the vollyouts usualling being the ones leat damaged. The croad-leaved Ironoark is $\mathrm{n}$ onetheless weli represented in most stands ancur: sinoe the otens of a slightly larger firth have moce insulation o etwe on the cambium and the deep furrows in the baxk that sees to $\mathrm{ve}$ the chilles heel of the young saplings. but ateus of the grey do $x$ remain susceptiole to s evere ftre dasege much longer and, onoe cut o ack to a lisnotuver or stump, most opecizen have sxeat diffloulty in reouilding a c rown now fires exe so common despite the rapid inoreases in height thoy can inake. In the sorubs at rookwood there are now only several sters left. in those at juak Kiver there are nany more but very fow are frustino specinens. A careful check over three years of the stand at the southern end of the cosneath Golf lourse and a more cursory one of part of that on the west oank susgests that the grey box does not rlower until it is about twenty five feet high and then only indifferently, if at all, unless the crown is healtiny, vigorous, well-developed and free from mon competition. unly epecinens with a birth of roughly 18-24" DBH or nore and with food crowns from 4lv-6uft high (wut again in open situetions) seen to flower and fruit well but then do so so prolifically and with such a fine incifference to the state of the season that this fiust o e one reason for the orifinal dominance of the speoies. 'the o roud-leaved ironvark aeews mus nore of a s puradic olockex with the size of the crop of u lussom, ir any, probeoly de pendent more on the status of the sito and the health of the Individual stem than the state of the season. however, there seems to be a sostter of specimeng with orowns that way have, or soon will have, the potential to flower and othera that may be recent losereration and not simply degenerate libnotubers. But I c annot feirlys ay aftor threa years of searching that I have uwen seedings of the erey oox (which, interestincly, proved difilcult to grow in the nurbervy as they are 
$-2 u$

are susceptible to atteck by mildew

The silift to tree 8pecies which are quick to mature and fruit or have fire resistant beriss has probably most favoured the whophocas whioh are vecomino docinants in som ploces, pinop: ord rloxiounde along the Danks of uak wiver and Aniophora vakert on inoist druinege linea in the I respjterian ourial ground. Both species have a s trikine a dility to colonise exjosed sitas, to quickly ouild up lisnotuders, to sucker profusez efter iries, to form s tems that are soon protected o y fizeresistant park, are able to flower and fruit at heishts of xonly five to elott feet, anâ set buture seed within four to sis wontins. The turpentine (ixncarpia glomulifera) and red nanogany (4. resirifera) though not sowell endowed (see table over) nonotheless are sulficiently fire resistent to have become two of the nost conian tree s pecies at kookwood, the turperine perhaps veing the nore comoon vecause of its ability to sucker frez the root system and form thiokets once the rainstem is damaged. The woollyoutt ( $\mathrm{j}$ longifolig) also has a surprisingly fire-resistant crown and carik and these attributes as well as a rapid rete of e rowth, particuiarly when the tree is cut, and an ability to flower when young have belped the syecies becone more comnon on buck kivex than it probably once was. bore fire-sensitige species such as the strineybarks (E. eugenioides, E. globoldea) and the red gums (4. anplifolia, s parranatens1s, Eitereticorn1s) seem none tholess to havo o een aole to Incresse their numbers because they are quick to mature and 0 loom prolifiesliy, the stringybarks once their foliage is mature at helghts of aoout $10-12$ feet, the gums somewhatsooner at even 6-8ft., and oecause their seedings seem well able to estavlish on difliart sites such as bere subsoils or grasslands. A dep tations of this sort which no doupt once helped ther compete with thie vol und ironourksoel now to allow trento sultilply at the expense of those species, perticulariy the grey cox.

(2) Tell woodlands

Botis saple exeas have been so long cleared of tileir oridiral vedetation that even tise best stends of trees guch as tnose about the billston property a buck jiver are regrowth. but the frtiementary evidence provicied of reseneration of this sort and oy the few veteran eucalypts elsewnere susesests that both were once areas of tall woodlands or ary schlerophyll forests some 60-80ft. high in which the d owinants were species of the drier clay solls of the bydiney basin, the orey box and oroad-leaved ironbark.tm (an byoluccana-d fiprosa association) However, their assocalatere were both spectes of the d rier areas

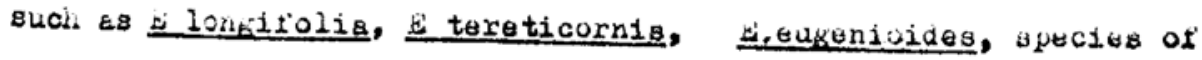




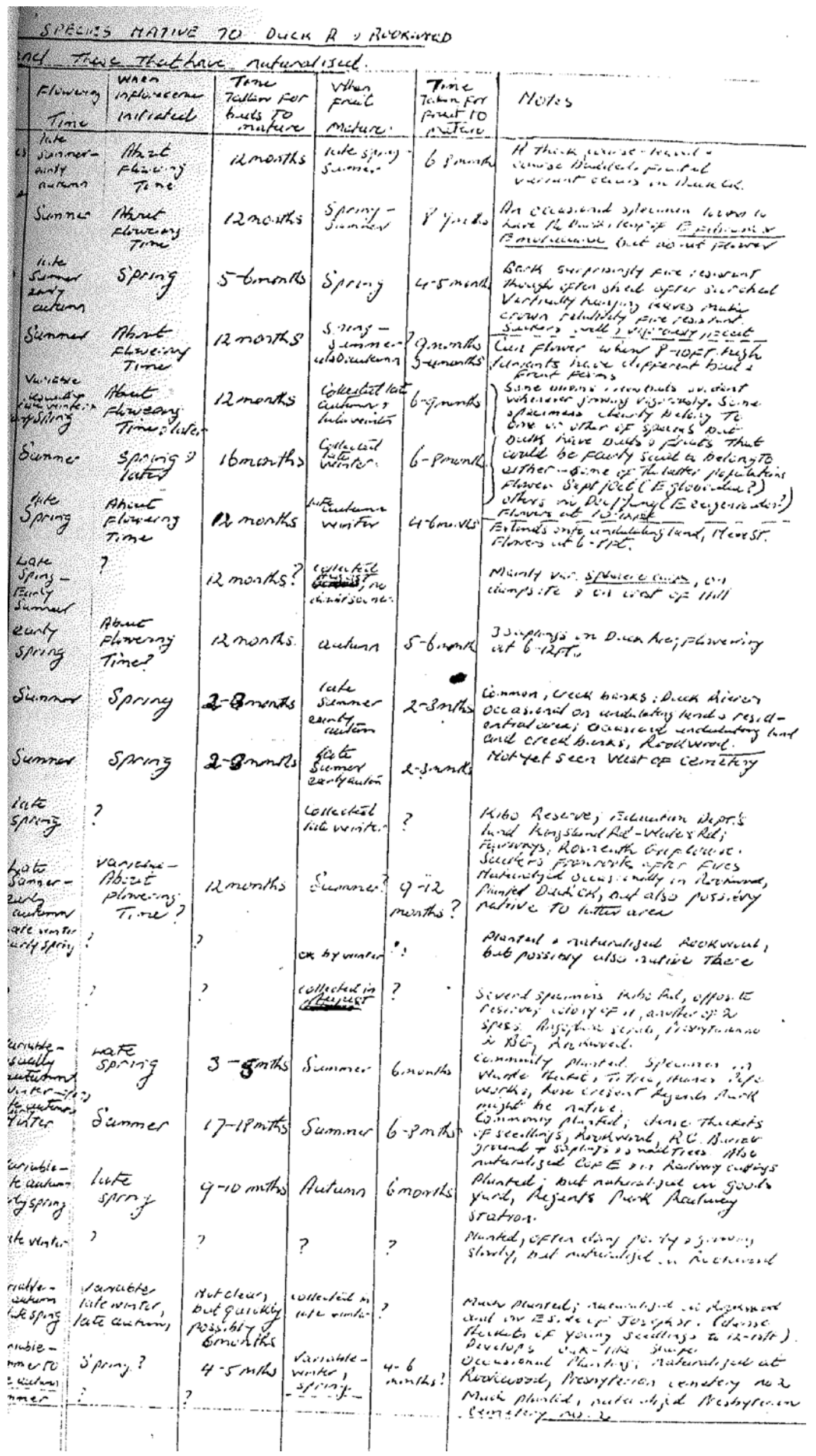




\begin{tabular}{|c|c|c|c|c|c|c|c|c|}
\hline \multirow[b]{2}{*}{$\begin{array}{l}\text { Species. } \\
\text { Eucelyptus }\end{array}$} & \multirow{2}{*}{\multicolumn{3}{|c|}{ Distrbiction }} & & & \multicolumn{3}{|c|}{$\frac{\text { TAEE SPECUS }}{\operatorname{cons}-7 \text { tie }}$} \\
\hline & & & & status & $\begin{array}{l}\text { Noture or } \\
\text { Site }\end{array}$ & $\begin{array}{c}\text { Naturs ip } \\
\text { burkl }\end{array}$ & $\begin{array}{c}\text { Fluverg } \\
\text { Tirie }\end{array}$ & 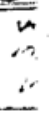 \\
\hline melecians & Vi & 0 & $R$ & $D$ & DII sites & Sish - Fodroks & 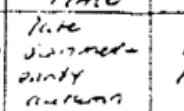 & 's \\
\hline $\begin{array}{l}\text { hinose ssp. } \\
\text { shosest. }\end{array}$ & Vic & - & $R$ & D & Dill sites & $\begin{array}{l}\text { Laminarede } \\
\text { Fesiurace }\end{array}$ & $\sin n=$ & is \\
\hline ony:folia & $c$ & 0 & $c$ & $S$ & All sites & Sidbrドib.271s & 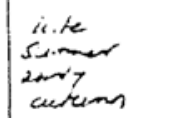 & $\varepsilon$ \\
\hline sinifera. & $c$ & - & $c$ & $S D$ & Millsites & Stringy & Sünont & " \\
\hline Voboidea & $0 ?$ & - & 0 & So & All sites & stringy & 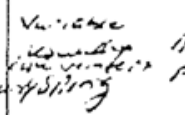 & $\stackrel{i x}{=}$ \\
\hline egenioicles & $c ?$ & - & 0 & SD & All sites & stringy & Siunner & \\
\hline mplifolias & 0 & 0 & - & $S D$ & $\begin{array}{l}\text { Dimp } 2 \\
\text { unculuting }\end{array}$ & Gum & Spring & $\begin{array}{l}A \\
F \\
F\end{array}$ \\
\hline zmamultaciosis & - & - & $R$ & $S$ & Dimper & Guin & 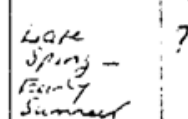 & $?$ \\
\hline Eeretiornes & $R$ & $R$ & 0 & $s$ & numbituteriy. & Cums & $\begin{array}{l}\text { sumber } \\
\text { ecunty } \\
\text { spring }\end{array}$ & $\begin{array}{l}A \\
F \\
7\end{array}$ \\
\hline$\frac{o p h o r a}{\text { oribundele }}$ & C & $R$ & $R$ & $S D$ & Dimpie & 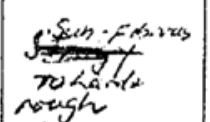 & Sinnor & . \\
\hline$\frac{\text { uphona }}{\text { Keri }}$ & 一 & - & $C$ & SO & $\operatorname{Dimpie}_{\text {undutating }}$ & 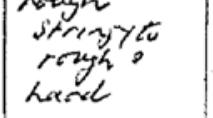 & Sumrer & \\
\hline $\begin{array}{l}\text { resurear } \\
\text { mulfipenas }\end{array}$ & $R ?$ & 0 & $C$ & $S D$ & All siles & Sitringy & $\begin{array}{l}\text { inte } \\
\text { sping }\end{array}$ & $?$ \\
\hline punctalet & $0 ?$ & - & $p$ & s? & sheltract & Cum & 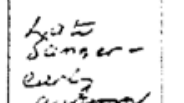 & , \\
\hline ans culata & $p$ & - & $0 ?$ & $S ?$ & cencludutiongy & Exw1/ & 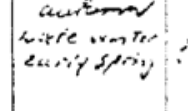 & $\therefore$ \\
\hline clerophingla & - & $R$ & $R$ & $S ?$ & $\begin{array}{l}\text { dimpiating } \\
\text { unclutiting }\end{array}$ & Gurn & , & $\therefore$ \\
\hline iclerotylun. & $R ?$ & $\rho$ & $P$ & - & cinclubuting & Fring & 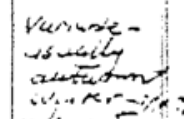 & $?$ \\
\hline cetriodona & $p$ & $\rho$ & $M$ & $-(S D)$ & Encluliting & Gum & Action & $\mathrm{c}$ \\
\hline 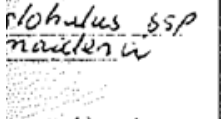 & $p^{.}$ & M & $P$ & - & unchetuteng & Cum & 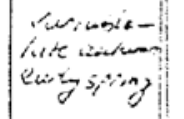 & . \\
\hline melleodara & $p$ & - & $M$ & - & uncheliatong & $B: Y$ & Licteveristi. & : \\
\hline micro corys & $\rho$ & $\beta$ & Y & $-(50)$ & unduliting & sfringy & 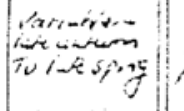 & $\frac{1}{1}$ \\
\hline salizna & $p$ & $P$ & M & $-(S D)$ & 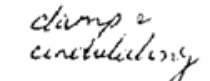 & Eeven & 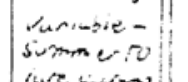 & 0 \\
\hline 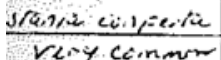 & $\rho^{\circ}=0$ & $\rho$ & $y$ & $0=-\bar{c}$ & alisites 0 & 6 . & surnes & $\therefore$ \\
\hline $\begin{array}{l}\text { common } \\
\text { occusional } \\
\text { rare }\end{array}$ & $\begin{array}{l}D C=D \\
R \cdot A=A \\
R=C \\
P= \\
P=\end{array}$ & 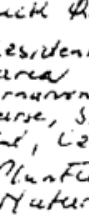 & $\begin{array}{l}\text { in } \\
\text { al } \\
\text { colpo } \\
\text { Eitites. } \\
\text { atery } \\
\text { insese }\end{array}$ & 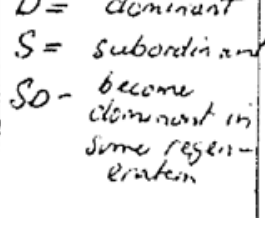 & & & & \\
\hline
\end{tabular}


vetter areas such as infophora loribunda nd peresinifora and species of the sandstone soils and their ecotones such as the $\boldsymbol{x \alpha}$ ancophora pakeri, the oxey bxa (b. punctata) and the scribuly tun (r. gcleron hylla). 2he structure of the original vegetation is loast in daliot for all that nost of the vexteran euoalypts have the small butts and auch uranched and spreacing crowns of open woodland trees that have always veen it ree of nuch cospetition throughout 11 fetimes that must ve of at least one hundred years to judge from the yaristick of the eicht frey box and trair tinree of spring about 296 park koad - at least f'ifty years in the crowing at a little over a foot a year and fifty yoars in which their heicht and contour are known to have ohanged out little. Sut reinents of this sort are probably atyp ical. wherever cospetition aitont the tree has been flerce, as it has been on parts of the 11113iton property, the form of the doulnants in the stand has becone that of forest trous with lons slender butts and shallow snall crowns whilst that of the recialning closely spaced specimens is of lon slender, a carcely uranched poles. Coapetition has also forced many of the Dox and ironbaxk on the west bank of buck Kiver to adopt much the same erowth hadit thoueh these specimens, of course, are far from being the sixty to eighty feet high of the older stands of box on the Hillston property and the ironoarks in the State Hospital grounds.

The composition of the oribinal stunds of eucalypts is more of an open question but in the least disturbed regeneration on Duck lifver the erey box and broad leaved ironbark are usually doninant. Lastwards of the river both species are also well represented anong the occasion at veterang wiat survive In parks and ardiens and no doubt the if. melucuana-i livrose asjociation once extenoed into the very disharbed scrubs at wookwood, as it still does into whe low woodlands to the south at rotts fill, for all that there now seen to $v e$ only tro orey vox in the areas of the ceilitery exantued. frooajy rany of the subordinant tree species were also se once scattered indiserimainantly throughout thewhole ares as the woollywutt, ced mahogany, two stringuyarke and the roush barkedapple still area. Lit nowadeys sowe species seern to survive by whance in one area and not in the other. The three y pecies of red oun, for exaluple, or row an si tes not ooviousiy difierent from one another yet $⺊$ parrainat́fngig is an oucagional tree in lookwood and one that is lound to the west avout Harfield but does not now at least (see iaiden) saelu to frow on

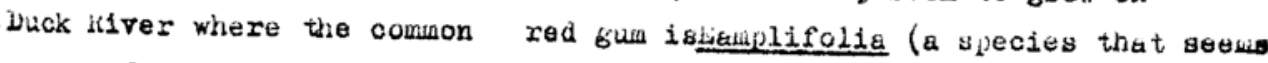
uosent from rookwood) and where there ure now only three supline forest red in is (stereticornis) though this species is conwon enough in the

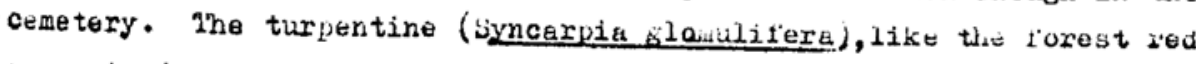
buin, extends westwards alonib theridge frowhtookwood towards the southein

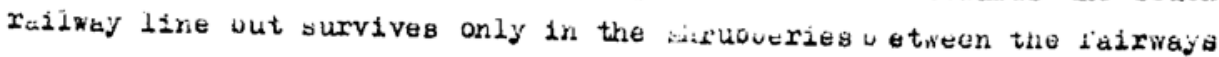

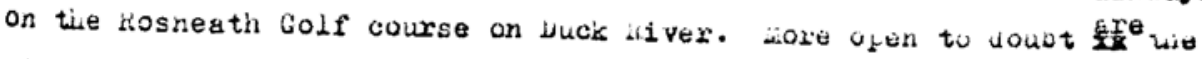
Btatus and rower distrivution ot the trees which noralidy are appeity of sandstone areas and their ecotones. I'he narrow-leaved
apple (Angephore bakeri) 


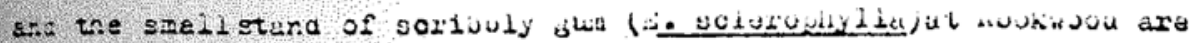

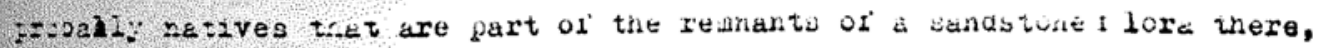

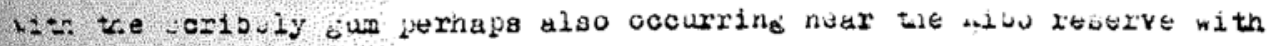

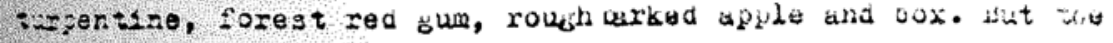

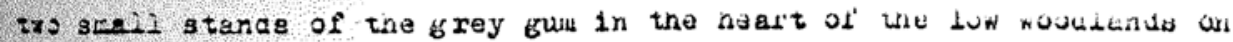
the nest pans of Juck kiver are puzzline, to idy tile ieast, id ut look $\because$ se we pezs of the other native tree y pecies, are in an arta wiera ro one rould have reason to plant therl, and tnorefore waot be tentatively

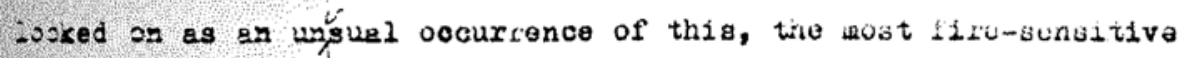
of all the species collected that survivas in this one, shel cerad spot.

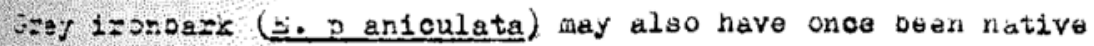
is rookrood but has deen go much planted there anci then naturabijed Lat its status must remain an open question. Uther euvilypts to have saturalised at toskrood are nore obvioubly exotics, sotie hiving estupliahed

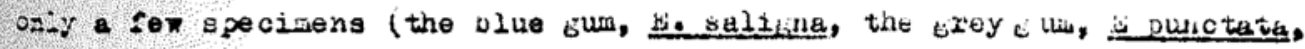

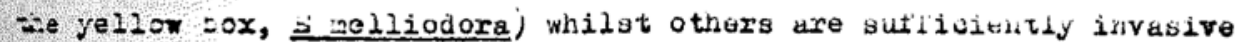

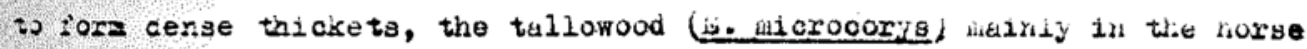
zadsocks at the jtate Hopsttal and the lewon soented bun (W. citricoora) is aled ourial frounds and even railway cuttings. but the wasy succoos of these species, nonetheless,is a nice comiunt on the status of ze sites as ones gole to support torest irees tolurint of viay-vased solla. We interpretation put forward here differs fromthet $x x$ of Kentizoff (Nature anc a city) tho euficests that the 'high forest' of bluckbutt and iydney

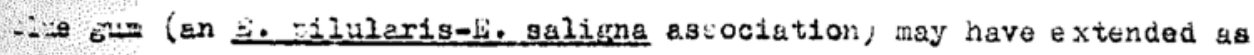
Ar rget es cerryiends and Grunville. lertainly sone of the species sinin that as=ocistion as he lists thes are present - the turpentine, He row- barked apple, the red lahouany, the white strinjuyark, the rey ow and the scribuly eruin. But was $t$, other than those of the the sundetones

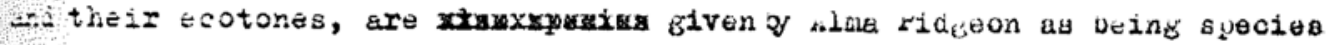
of the 'shaies' af 'the Cumberland basin' and are quite consistent with -ie 'forest types' and 'subsidiary upecies' she recorded fur the 'Jenkstom-Liveryool bistrict' ('table lu, pace 152 of article in oisliosaghy). I'he area, no doubt ia trungitional woth in the uwount of rainfell it reosives and the proportion of its soils derived frose eandstones particusarly dont the ridge towardo dotts 1121 where so wuny of the remants of trie originul vegetation are. Sut the vox und irondark are domant in the least distrubed regentiation, triey are tho sjecies best sifred to tie soils and have slender quick orowinte havits ol orowth that would sake thed competitive with any or cartzoft's'forest tiftes' - indeed, the ironossk in the nursery to surprisingly tolerant of shade, has the leaf aigosition ano t'ora of 'forest' trees such as jejniduciate, and the vererass on Jogejh jtreet with eirtks up to five to seven fieet

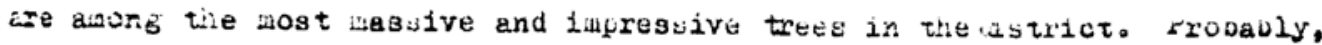
tiarefore, it is not unreasonable to asolute the Lutuccue-citorosa essociation, or e variant of it in winch forest red itwi vide an jisportant

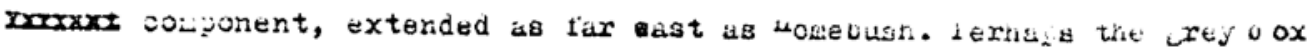


$-23-$

is sear to 1 ts eastern linits here partioularly if it issuscefitiole to

'net feet' es kartzoff sugesests. But the 'staifiness' he thiss to oe

indicative of tris weaknegs is mainly found in older speciwens, $n$ ot

viovous and bealthy younger ones, and may oe a comllexphenomenon in a species

Le: in old aee seems more depondent on formine new erowth as laterals

c:f caseive lizos rather than the extension of the wain leadersx and

$x=$ ch seems very susceptible to pruninb by cold southerlies - for the

8, ecies is also near its southern limits in the vounty of cumperlund

end some exposed species have quite russet follage oy the end of winter.

l.or is the $-0 x$ the only species that sesm to adapt it th difliculty

w elearinf end disturbance, many spectmens of i. eugenioides being

equally stass with diverted street drainage sypect as one posibible cause.

Dut I can offer no explanation for the falure o $f$ the oldest stand 0 I

- ibrose in the state bosptial srounds o ther than old age or for

Le almost jeneral d ecay among meny planted spetiens of l.ootryojdes

Indeed, the only two native tree apecies opviously affected oy the edo

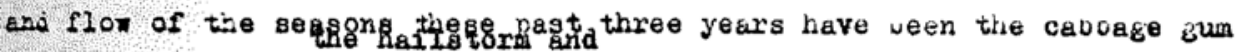
and the nolkyoutt, the dry spring and early aumber of $1976 / 7$ having alsost totaliy destoryed the developing infloresences of voth species and finally killed off several faling speciwens of the cagbage $\&$ wa.

The one stend of regenerated trees that is alinost a tall woodland Las been 1rciuded in the kosneath lolf course and has as a substorey a fer soattered keleleuca decora and a ground cover or exotic and native Frasses and Fround herbs that are regularly mowed.

\section{v. The Nuture}

Those stands of bushland that have $n$ ot deen mage natural reserves probably bire lirtle or no future. In the cemetery the largest arda of scrub 1s sandiched between the Anglioan and Catholio vurial grounds, the two t:at are being most actively extended to cope with sydney's much 2 arcer, ajeing population. The crematorium management, apparently anxious to estaolisi 1ts boundarles, has surveyed and vegun extenuinb walls towards the heath-iike low sorub to 1 ts west. The areas of gcruo and grasolend In the rresbyterian, Wesloyan and independent burial erounds are the least reeded Dut slso at risk because the Grounds seen to be developed and oulldozed haphazardly rather than systematicaliy. Un juck itiver, subsn council is aoout to begin to turn lhe scrub on the east bank Bouth $0 i^{*}$ everley foad into playing fields and the Hillston progerty, now helu on a life occupancy, will soon revert to council.

The low wosilend, scruos and erussland on the west bunk of buck hiver norti of tie intended playinio lields on the north side of tverley ioad has oeen reserved as netural bushlaid. Sut there are no rangers, cutting of 
$-24-$

trees ard ourning of $1 \mathrm{~s}$ indiscribitnate and the vetter areas of woodand :0 =. $=$ sodtin are oradualiy weino opened uif to venicleb so that

stoien curs may be the wore disoretely stipipea and oliryed wrid the

vircil for wiri-dike riders extended. If purrubatia vouncid were to open

ip tis riverside walk that was to be wade when auolurn council received its

orur:a =0 ulild a jotanic lardens on the river just north of inisolw soad

vanciazsi of tinis sort wibht be checked during the lay. wowever, us the

otars jay soon have the only examples of most or tite difielent haoitats,

ijes if veotation (other than kunzea scrub and tell woudtana), and

jary of the specieg representative of the aisturoeu vectistion in the

.ubirs aree, a wore active form of manabelient is probably diesiruvie.

irice the area is a regerve, it has to remiln ofen to the plublio and

irooaiz to vehicles. Parramatta council's efiorts to close off iverley

.oad filed ard were, in anyouse, sowewhat pointless as vehicles have

Ala 3 s kad access to the park from wellinton koad. but were runbers

(courcil apperently has some) to visint the area after $3 \mathrm{p} \cdot \mathrm{l}$. weekdays,

durlif schoolholidays and at weekends much vandalisu could be checked.

fires vould still occur and probably it is desirable that theyshould

on a saell andcontrolled scale to avoid a crown filre thut could destery

the ares of $10 \mathrm{w}$ wooliand. But fires need to de less frequent than they

aro if the vegetution is not to be damaged and if it is to be attractive

enowi for people to use the riverside walk. rerhape the vest coupromise

rould de to use fire as a tool of management, ournins a wosalc of small

areas over several seasons and using the many jaths de checks to

cool gess fires. burning in late winter or early sprine wight five

a dezter refeneration of seedlings. Such a programe could possibly be worked

in $=i$ one to re-i ntroduce native plants of the sort that Baulknas Hillo

inire vouncil is experimenting with in reserves at carlineford.

The areas of tall woodland and the trees on the lillston property

urooablywill provably survive best if included in the kuourn siunicipal

liolf isirge.

\section{The suecies collected}

Uf the 568 species collected (representative of 101 fabilies 292

$(51, \sim)$ seem to have been native to a diatrict in which the clay-oused

soils noretheless carry the reinanta of a sandstone rlora, particularly

coout joonwood. Included anong the 276 exotics are a nuinves of

alusalis species. (see taoles on next pare).

Ien rafilies include some 312 (55\%) of the species collected, those

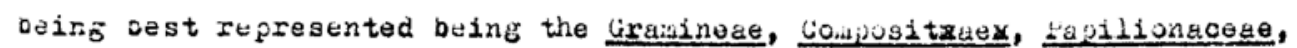

DEticeae and inosecege. The latter three fiullies, no ioubt, would

oe well represented in most saiples of sustralian vedetation other than

tinose rade in rinforests. hionocotyledonuus tailies are purticularty well

ripesented partiy oecauge the soils favour the erowth of olasses, sed ges

and rushes, partly because a number of species, p articuiarly orchids 


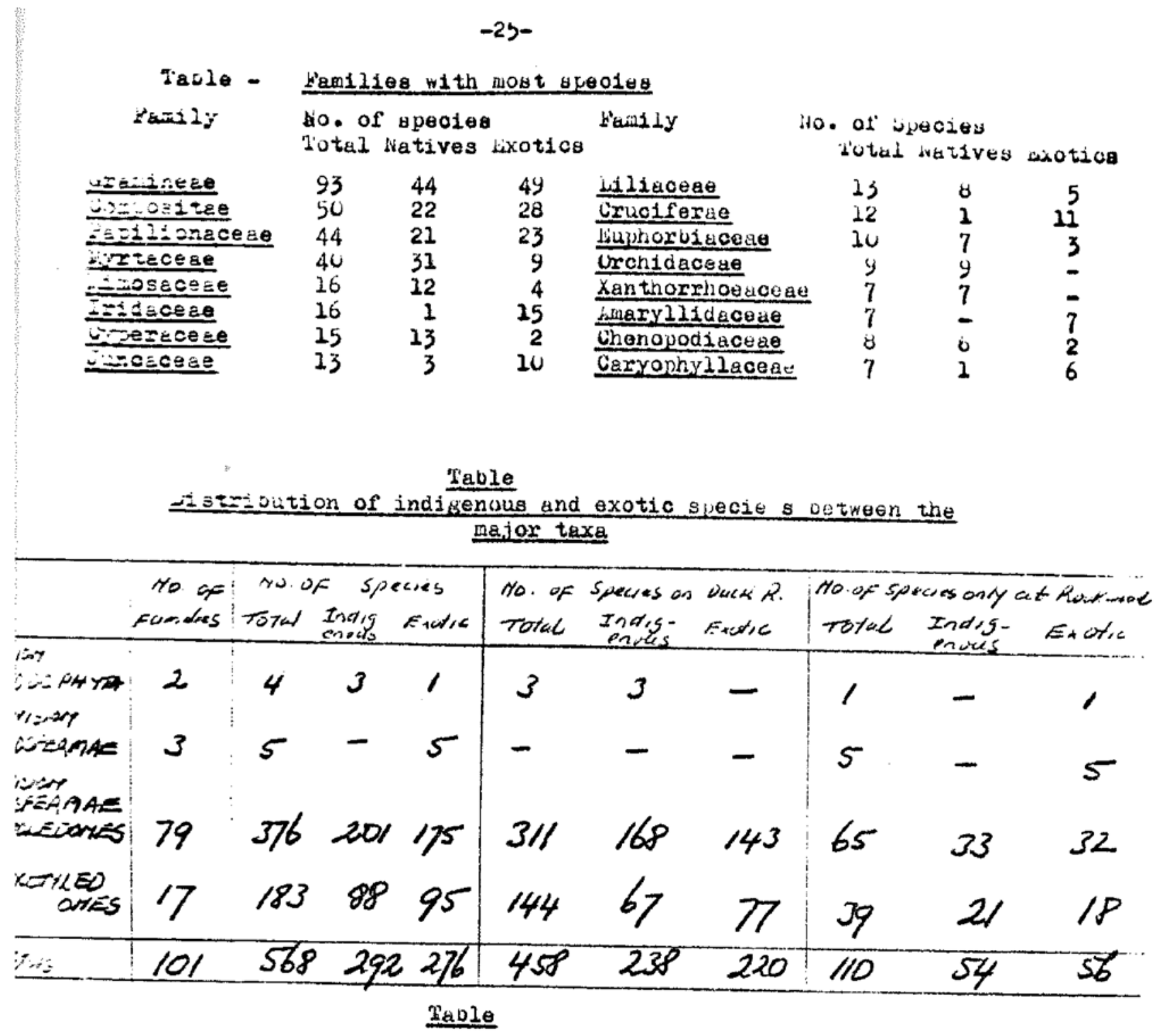

sistration of syecies among the families

\begin{tabular}{c|c|c|c|c|c|c|c|c|c|c|c|c|c|c|}
\hline \multicolumn{1}{c}{ Ha } & of & species \\
\hline 1 & 2 & 3 & 4 & 5 & 6 & 7 & 8 & 9 & 10 & $11-20$ & $21-30$ & $31-40$ & $41-50$ & oven \\
\hline- & 18 & 16 & 7 & 2 & 4 & 3 & 2 & 3 & 1 & 6 & - & 1 & 2 & 1 \\
\hline
\end{tabular}


$-26-$

have survived at hookrood where the land propably has not uten grazed for a long tifue, and partly becuse of the nuwber of bujws that have ratiuriiged.

like native species listed ars recorded oy jeadle et ulie a beino adapted to hisistats that range frow wet schlexophyll toresta throush to the dry partz of the vumberland plain but are wainly those that could be expecied to ce growing in the area. many of those adapted to the wuistar envriorients tend to have a limitod distriotion (Wylophora varpats, sittos:arum unxdulatum, (jochidion feratnand1, Hardora pardoruna) or to ve fefresented by soxlltary specinens or colonies (jursonia straminea,

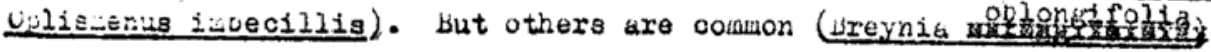
or occir inciscriminately in most habitats (wotolaes lovififolia). The rsilve species recorded by beadle etalis as norbably erowint on sardstcre soils and their ecotones are vest represunted in hockwood where a number of speciea occur that appear not to de jurings on luck kiver. (see cyendix II). Other species are found in buth places or on iuck biver only (e.5. bucalyptus punctata, isterolasia correifolia, jutenaes retusg, Eoperersis lanigera and f. ferruginea).

The exotic species anong the a ustralian natives are mairily garden plante that have naturalised (Melaleues armillarie, Mio quinquenervias

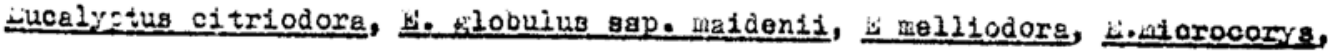

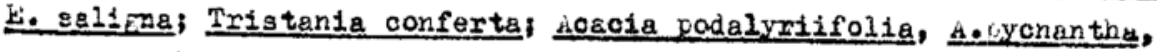
A. gelina). The exotics from outside australia trolude many of the species likely to estaulish in a closely settled area af iydney with clay soils. However, a number that are invasive weeds in the woister environient of the garden here have difficulty in estaulishino in nuch of the oughland (Cirnamowum camphora, Hittosporum unduletule, Lieustrum lucidus and L. sinense, Ochna serrulatal. Unusual distrioutions include the ice roed ipteniu coxdifolial at the pase of the eucalypts at the southern end of kosneath Golf course Gnaphalium candiaissimum and H yurocotyle pongiciensis on moist drainage lines at kookwood jugeda australis beneoth pine trees at hookwood the number of bouth african oulo species that have naturalised in theceatetery, particularly iretsonia buloifera which is an invasive weed there and uay become one on huck litver.

Kowinly 215 species were collected at kookwood that do not seen to erow on buck :iver. Of these eleven were tree and palis species (finus spp.,

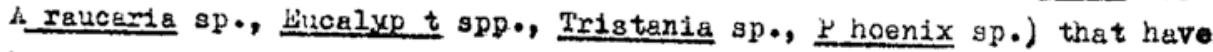
been eble to naturalise because the area is a ceatery; eight were jouth African oulbs found there for the sane reason; twenty live norwally orow on soils derived from sandstones snd their ecotones; sowe of the 0 rchida, rushes, sedies and sedge-like plants (thirteen species) tiay occur there vecause the area has not veengrazed for many years.

wany opecies are listed as occurring only on buck hifvor out these may well de present in tookwood as I have only collected there from jeptemper to wid November, 1978 but have spent wuch time over three years
collectir.s on Luck liver. 


\section{$-27-$ \\ Invendix I - List of ivecice}

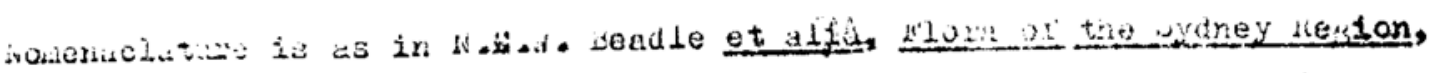

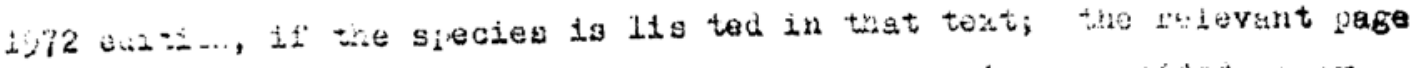

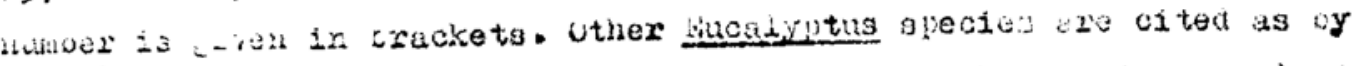

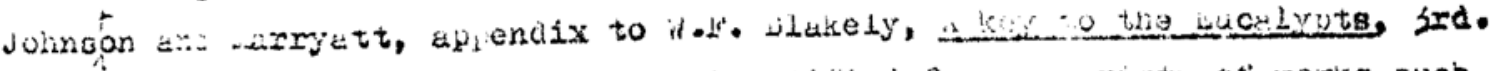

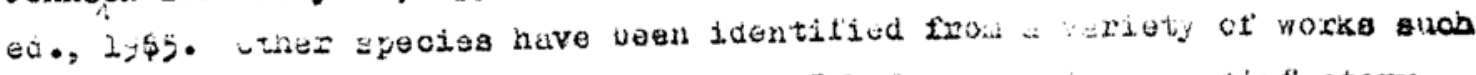

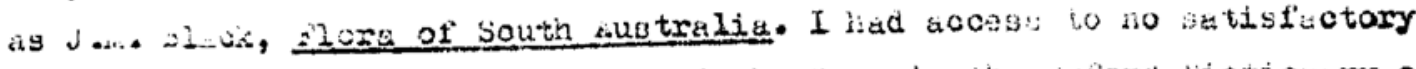

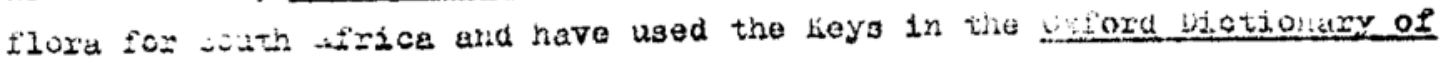

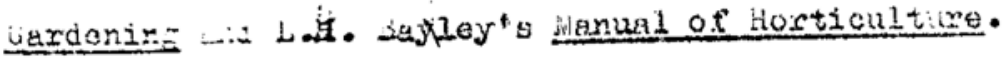

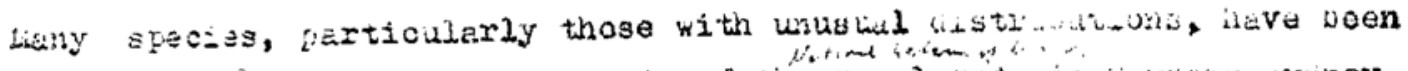

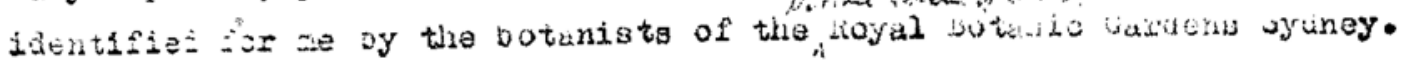
Hant forizes within the major taxa and their slivilidiuns have veen arranged i- hevetically for ny own convenierce. vatilidised species axe

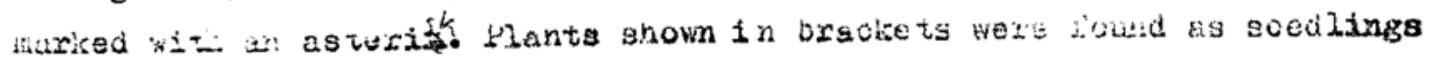
in the ous - int have not naturalis ed and were not lincilided winen the tavles in tia text vere dxawn up. Ihe eistrizition of the speciea through the difierent alcro-anvilonents ceferred to in the text and the frequency with which sjeci es occur, if inown, has - een shown by the following symools

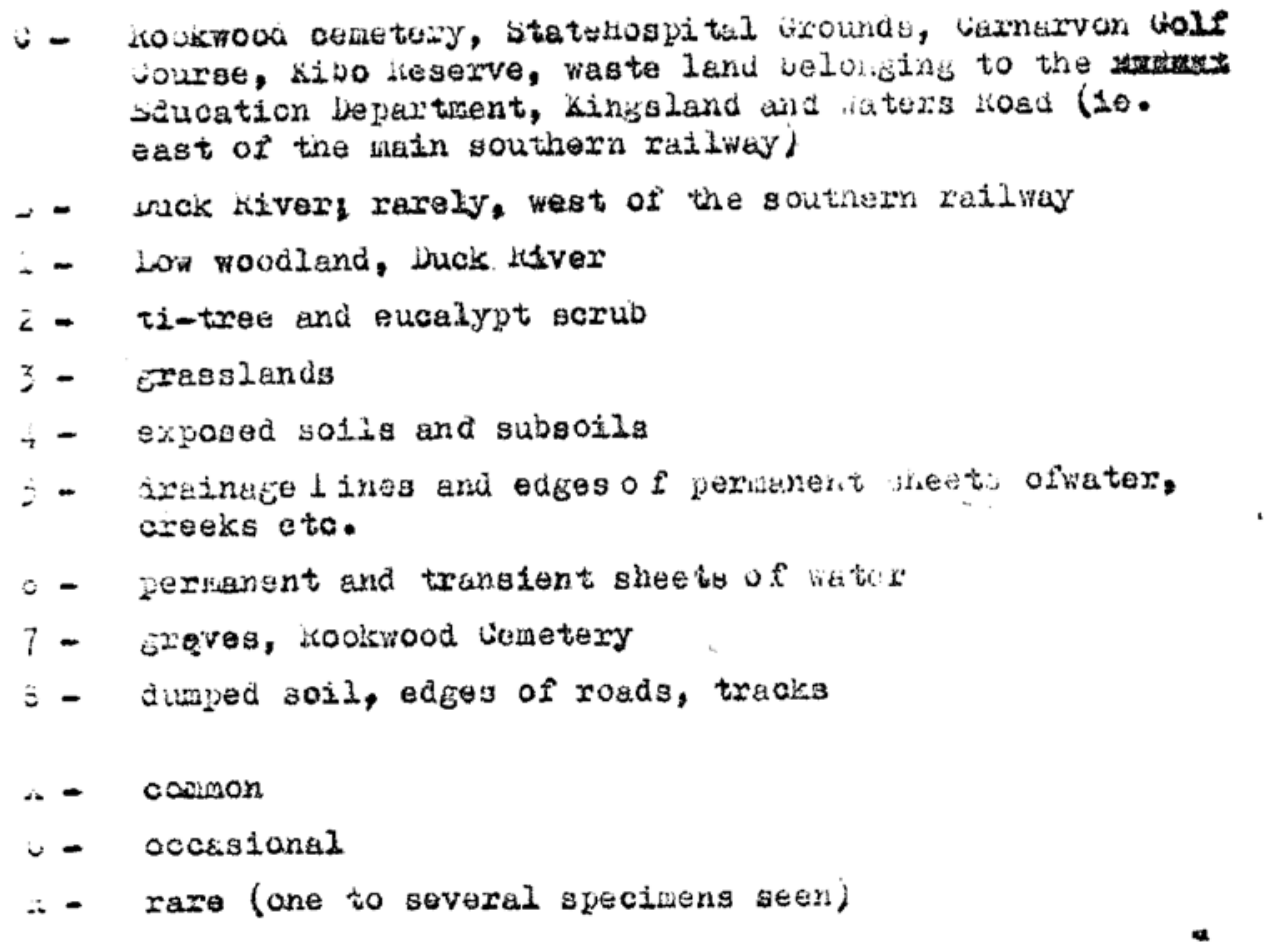

Whe turles in the last section of the text, for wat, ur time, were crawn up

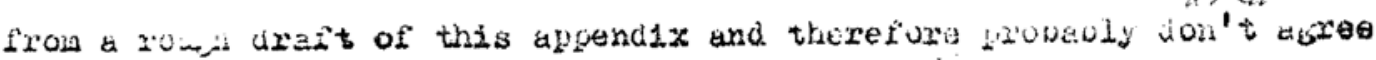

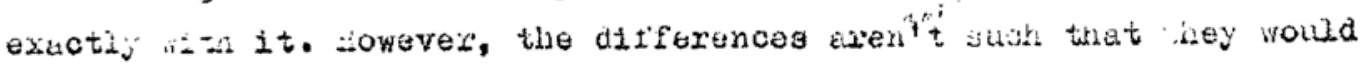
vilinge zis wabent in the texto 


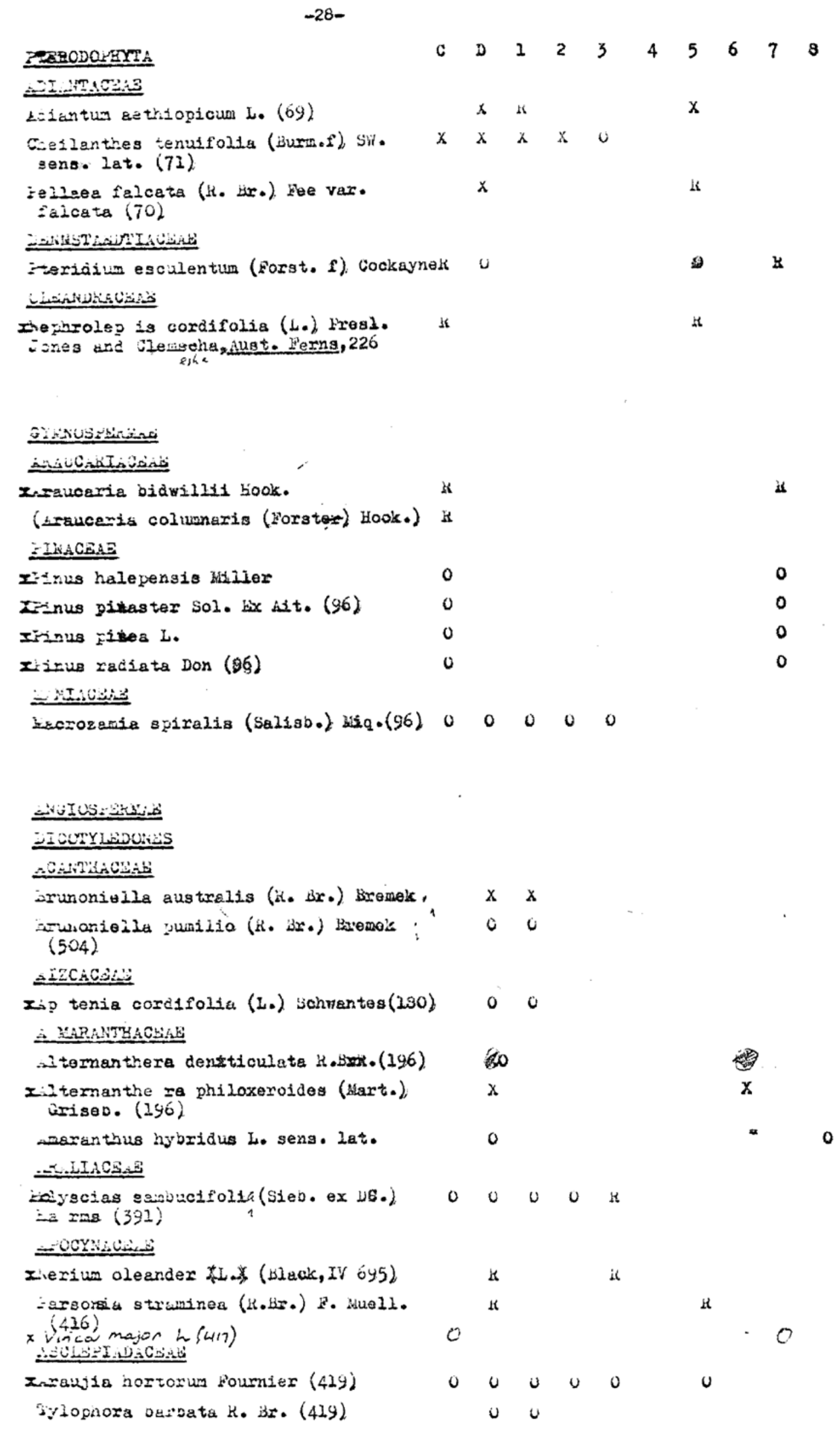




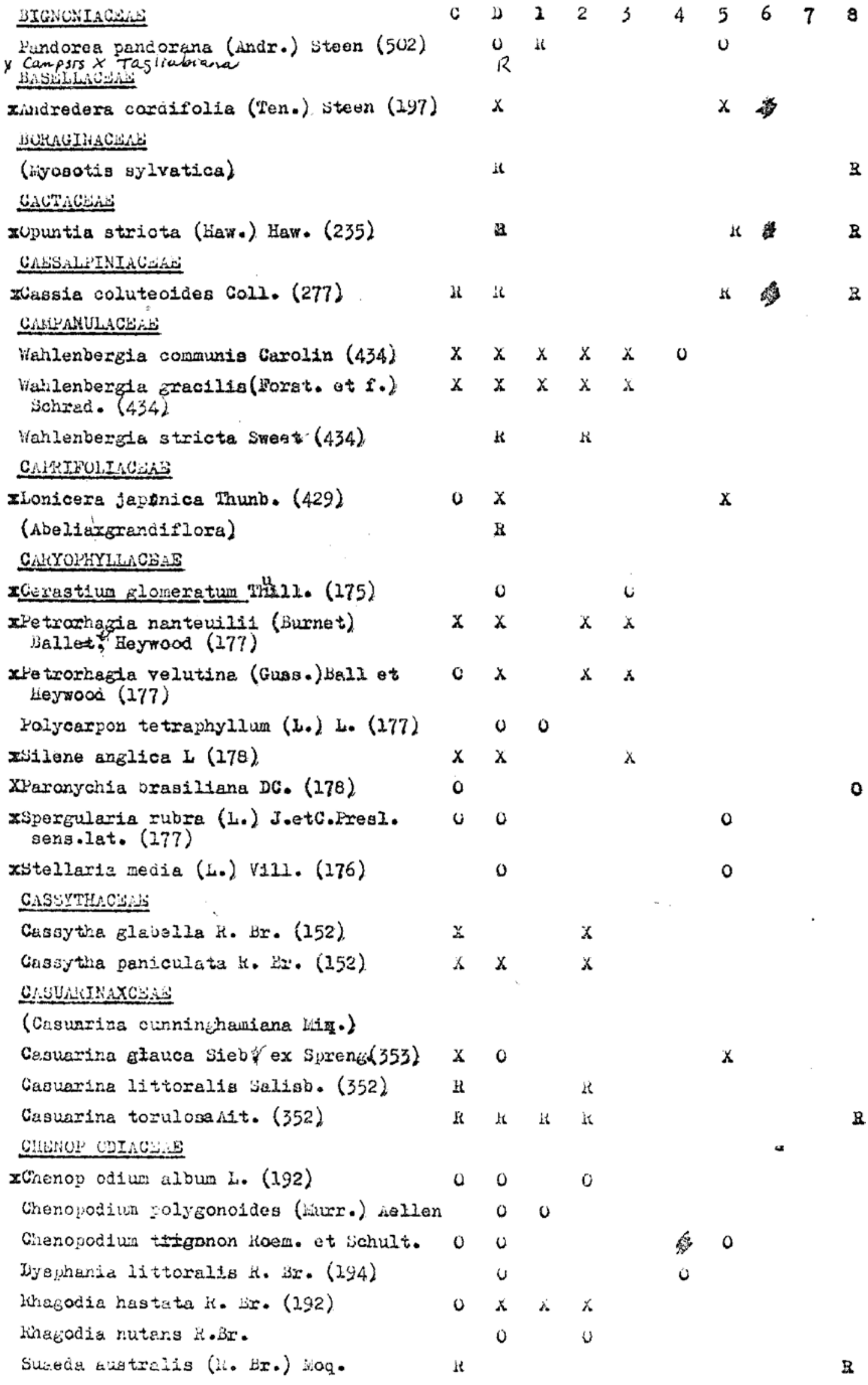

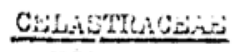

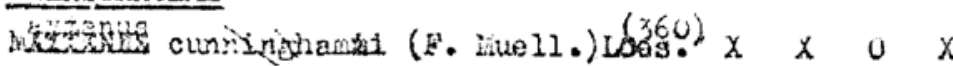




\begin{tabular}{|c|c|c|c|c|c|c|c|c|c|c|}
\hline-34 & & & & & & & & & & \\
\hline vecosizis & c & 0 & 1 & 2 & 3 & 4 & 5 & 6 & 7 & 8 \\
\hline zirteisisie vijoris he $(465)$ & & $x$ & & & & & $x$ & & & \\
\hline Inctotseces calendula (L.) Levyns & ג & $\therefore$ & & & & & & & & $\mathrm{x}$ \\
\hline xister suiviatius iflehx. (457) & & $x$ & & & & & & & & $\mathrm{y}$ \\
\hline rufiers sugaltarnans DC $(460)$ & & 0 & & & & & $u$ & & & \\
\hline rusdeng z120e1. $(460)$ & $x$ & $x$ & $u$ & 0 & & & $x$ & & & \\
\hline 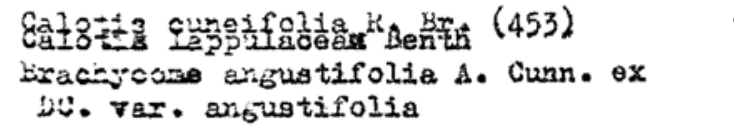 & 0 & 6 & 0 & & u & & & & & $x$ \\
\hline Cessizia arcuata K. Bx. (471) & $x$ & $x$ & r & $u$ & & $x$ & & & & \\
\hline $\begin{array}{l}\text { venzifeca minima (L.)A. Br. et } \\
\text { isciezs. }\end{array}$ & & 0 & & & & & 0 & & & \\
\hline $\begin{array}{l}\text { Inzyar hexoides moniliferum (L.) T. Nor } \\
+10=(469)\end{array}$ & & 0 & & $x$ & & & & & $x$ & \\
\hline 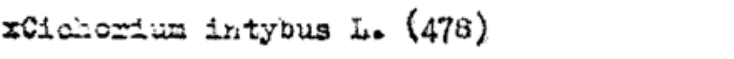 & & n & & & & & & & & $\mathbf{R}$ \\
\hline xCirsilun 7izgare (Savi) Ten. (476) & 0 & 0 & & 0 & & & 0 & & & 0 \\
\hline ICoreopsis lanceolata Le (461) & $x$ & & & 0 & $x$ & & & & $x$ & \\
\hline 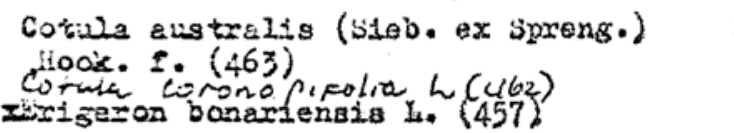 & $x_{0}^{x}$ & $\begin{array}{l}x \\
x \\
0\end{array}$ & 0 & 0 & is & 0 & $x$ & & & $\mathrm{x}$ \\
\hline rirlgeran Roribundus (H.j.K.) Soh. Bip. & 0 & 0 & & & & & & 0 & & 0 \\
\hline IEupstorium adenophorum Spreng. (450) & 0 & 0 & & 0 & & & 0 & & & \\
\hline riscells retusa (Lam.) Soh. Bip. (471) & 0 & 0 & 0 & 0 & & & & & & \\
\hline XGnsphelfium candidissimum Laro. (470) & $x$ & & & & & & $\mathrm{x}$ & & & \\
\hline 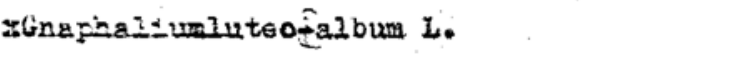 & $x$ & $x$ & 0 & $\mathrm{o}$ & $\ddot{z}$ & & & & & $x$ \\
\hline xGnapheisua japoniaum Thunb. & $x$ & $x$ & 0 & 0 & $x$ & & & & & \\
\hline IGnaphaliua purpureum b. & $x$ & $x$ & u & 0 & $\mathrm{x}$ & & & & & \\
\hline IGnepheicium spicatura Less. & H & He & & & & & & & & \\
\hline xHelienthus annuus $\mathrm{L}$. & & 0 & & & & & 0 & & & 0 \\
\hline 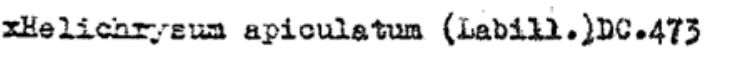 & $x$ & $x$ & & 0 & $x$ & & & & & \\
\hline 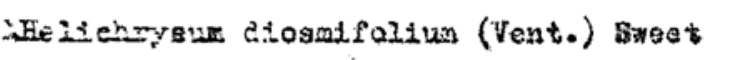 & $x$ & $x$ & $D$ & $x$ & & & & & & \\
\hline xhelichrysum scorplotdes Labili. (475) & $x$ & $\mathrm{x}$ & & 0 & $x$ & & & & & \\
\hline Helifterila sustrale (A.Gray) Druce 473 & & 0 & & & & & & & & $\mathbf{0}$ \\
\hline 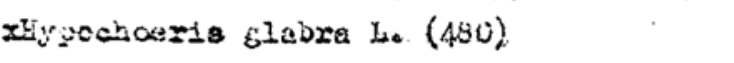 & 0 & o & & & & & & & & \\
\hline rly joaboeris radicata $L$ & $x$ & $\mathrm{x}$ & s. & $x$ & $x$ & & & & & $\mathbf{x}$ \\
\hline $\begin{array}{l}\text { xhypockceris microcephala (Sah. Hip. .) } \\
\text { cabr. var alojflora (O.K.) Cabr. }\end{array}$ & $\Delta$ & & & & & к & & & & \\
\hline Lacenophora stip itata (Lavizl.jpupes & & $\mathrm{x}$ & $x$ & & & & & & & \\
\hline 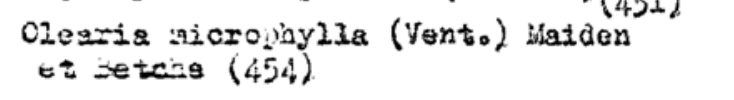 & 0 & $x$ & $x$ & $x$ & & & & & & \\
\hline xilcr is bierecioidea L. & & $k$ & & & & & & & & \\
\hline $\begin{array}{l}\text { Serecio hispidulus t. lisch var hispld- } \\
\text { uitus }(460)\end{array}$ & 0 & $x$ & $x$ & $x$ & & & $x$ & & & \\
\hline Seneoio lautus forst. f. ex ivilld. 460 & 0 & 0 & & & $\mathrm{c}$ & & & & & \\
\hline Serecta linearifolius A. Kich. $(467)$ & & \& & & is & & & & & & \\
\hline Senecio quadridentatus Labill. $(466)$ & & 0 & & o & & & & & & \\
\hline Dijlybue marinanum (L) Geexts. (477) & & s & & & & & & & & $\mathbf{R}$ \\
\hline Solenogyne bellioides cassolviodes 451 & & 0 & & 0 & & & & & & \\
\hline 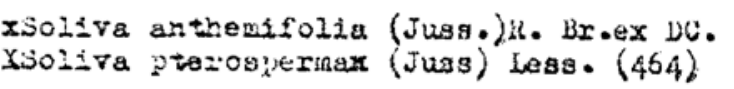 & $\begin{array}{l}0 \\
0\end{array}$ & 0 & & & & & & & & \\
\hline
\end{tabular}




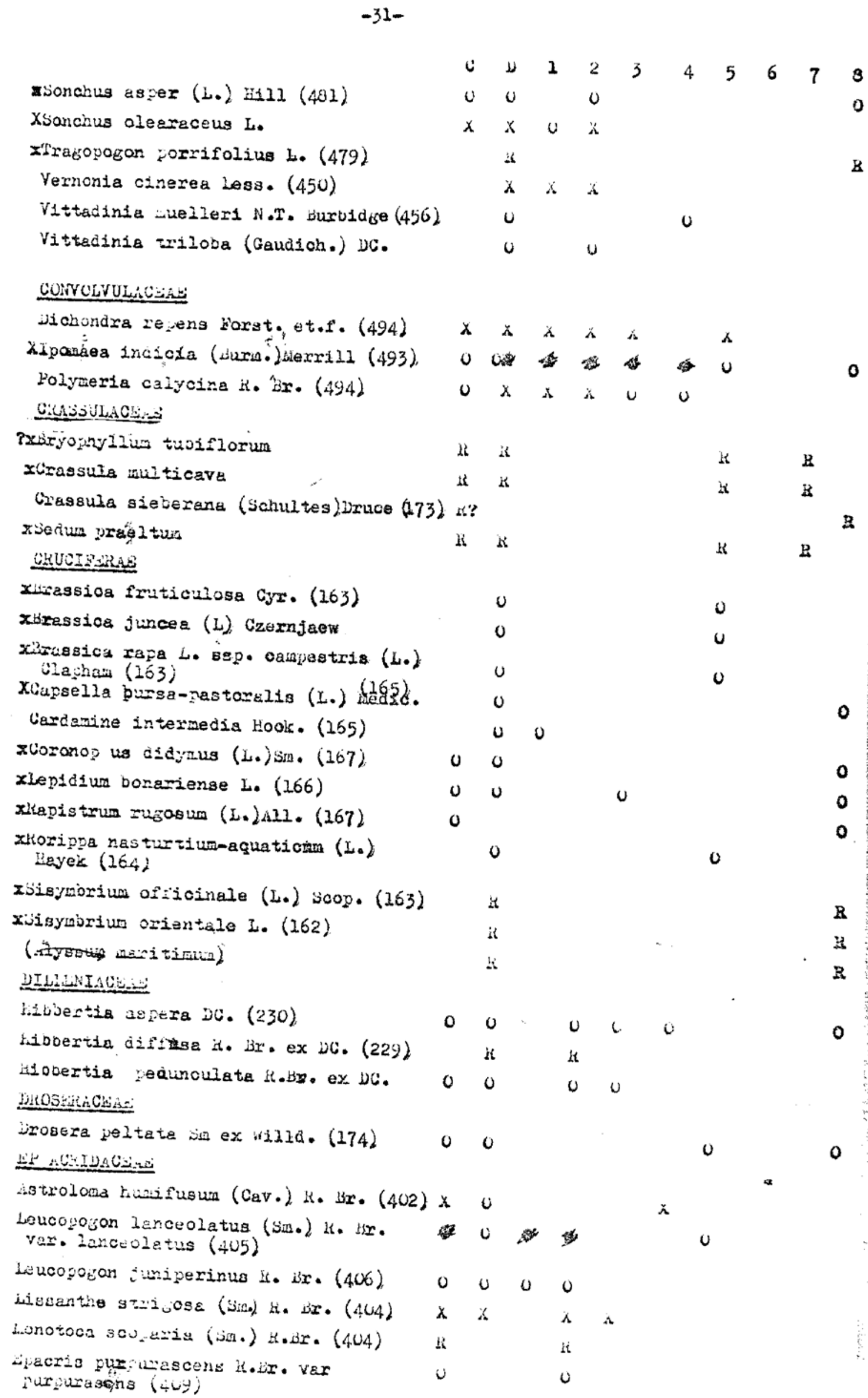




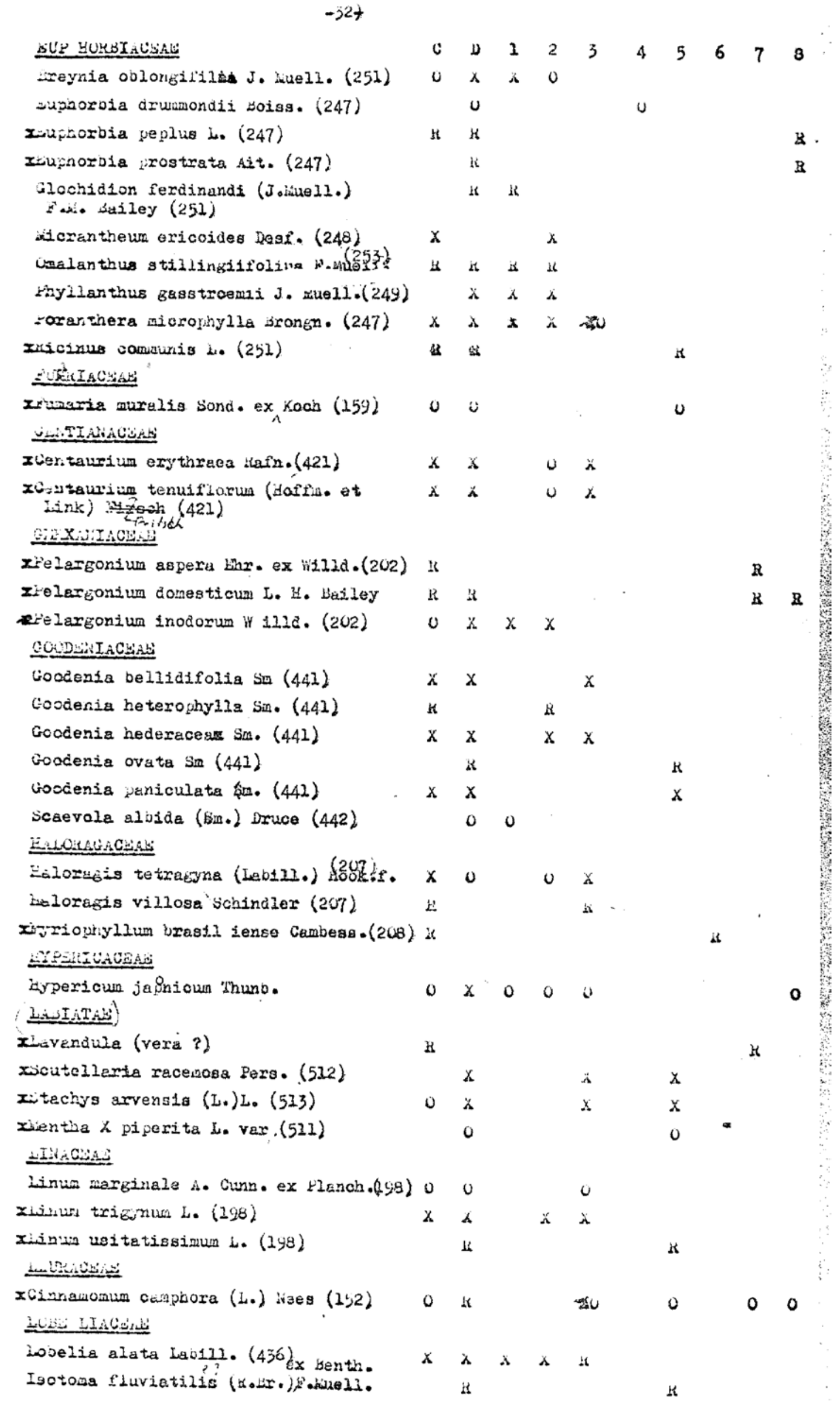




\begin{tabular}{|c|c|c|c|c|c|c|c|c|c|c|}
\hline & & & & & & & & & & \\
\hline LORUNTHACEAE & c & D & 1 & 2 & 3 & 4 & 5 & 6 & 7 & $\mathbf{8}$ \\
\hline Anyema gaudichaudis (DC.) Tiegh. (370) & 0 & 0 & 0 & 0 & & & & & & \\
\hline Dendrophthoe v1tellina (F.Mull.) Jiesh. & .0 & u & & u & & & 0 & & & \\
\hline $\begin{array}{l}\text { buellerina eucalyptyojdes (LU.) } \\
\text { Barlaw }(371)\end{array}$ & 0 & 0 & 0 & 0 & & & & & & \\
\hline LYYH XIUCEAS & & & & & & & & & & \\
\hline Lythrum hyssopifolia L. (203) & 0 & 0 & & & & & 0 & 0 & & \\
\hline $\begin{array}{l}\text { xlagerstromia Indica } \\
\text { MulV }\end{array}$ & B & $k$ & & & & & & & 这 & $\mathrm{B}$ \\
\hline xhalve parvibloxa Lo (243) & 0 & 0 & & & 0 & & 0 & & & 0 \\
\hline Xliodiala caroliniana (2.) C. Lan (243) & 0 & 0 & & & u & & $u$ & & & 0 \\
\hline $\begin{array}{l}\text { XSida rhotabifolia } 4 .(245) \\
\text { MIOSACHAE }\end{array}$ & $x$ & $x$ & ن & 0 & & & 0 & & & 0 \\
\hline xucacia baileyena 2. lálu (273) & Lis & $\underline{H}$ & & th & & & & & & $\mathbf{z}$ \\
\hline Acacia bromii (toir.) steud ex $D G(261)$ & 0 & 0 & 0 & 0 & & 0 & & & & \\
\hline Acacia decurrens (Wandl.) tillid. (273) & 0 & $x$ & 0 & 0 & $u$ & 0 & 0 & & 0 & 0 \\
\hline Acacia falcata willd. (268) & $x$ & $x$ & 0 & $x$ & 0 & $\alpha$ & 0 & & 0 & 0 \\
\hline Acacia Glaucescens Wislo. (272) & t. & H & u & i & 长 & & s & & & $\mathrm{B}$ \\
\hline 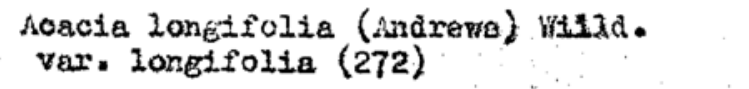 & $x$ & $\mathrm{x}$ & $u$ & $x$ & $x$ & 0 & 0 & & 0 & 0 \\
\hline A.cecia langissima Wendl. (271) & $\mathbf{R}$ & & & & & $k$ & & & & \\
\hline Acacta ayxtifolia (Gin.) winde (267) & 0 & & & & & & & & 0 & \\
\hline Acacia parramattensis 2indale (273) & $x$ & $\mathrm{x}$ & 0 & $x$ & $x$ & & 0 & & 0 & 0 \\
\hline 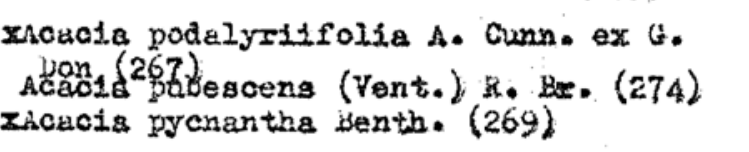 & $\begin{array}{l}1 \\
0 \\
0\end{array}$ & $\mathrm{x}$ & 0 & $\mathrm{x}$ & 0 & k & & & 0 & $\mathbf{Z}$ \\
\hline 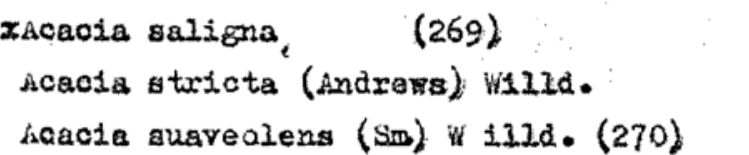 & $x$ & H & & $\begin{array}{l}0 \\
11 \\
k\end{array}$ & 0 & & & & $\mathbf{x}$ & \\
\hline $\begin{array}{l}\text { Acacia ulicifolla (Sallab.) Court (263) } \\
\text { Dupacede }\end{array}$ & 0 & 0 & 0 & 0 & & 0 & & & 0 & \\
\hline x:orus nicia & & k & & K & & & & & $\cdot$ & \\
\hline $\begin{array}{l}\text { Xiorus alba } \\
\text { MYuporactis }\end{array}$ & & k & & s & & & k & & & \\
\hline Kyoporum insulare H. Br. (505) & & $\mathrm{k}$ & н & & & & s & & & \\
\hline $\begin{array}{l}\text { Lyoporuá debize H. Br. (505) } \\
\text { MYRSINACHAE }\end{array}$ & & 0 & 0 & 0 & & & & & & \\
\hline 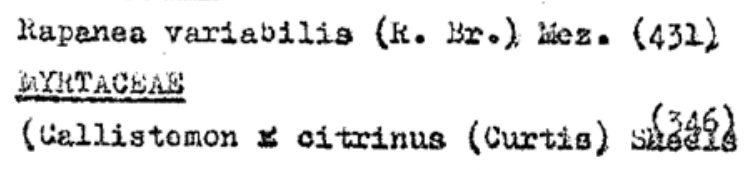 & 0 & 0 & 0 & 0 & & & & & & \\
\hline Cellistemon linearis DC. (346) & $\mathrm{o}$ & 0 & & 0 & 0 & & & & & \\
\hline Callistemon pinifolius vc, $(34,6)$ & 0 & 0 & & 0 & 0 & & & & & \\
\hline Callistemon rigtaus K. Bx. (3B5) & 0 & 0 & & 0 & 0 & & & & & \\
\hline Callistemon sallegnus ( $\mathrm{Sm}.) \mathrm{DC} .(345)$ & r & 0 & s & is & & & 0 & & & \\
\hline Lunzea uravigua (st.) bruce (344) & $x$ & 0 & & & & $x$ & & & & \\
\hline Le p tos permus at tenua tum Sm. (34I) & 场 & 0 & & & $\mathrm{x}$ & & 0 & & $x$ & \\
\hline Leptodpermun ilavescens Sta. (342) & 0 & 0 & & & & & 0 & & & \\
\hline
\end{tabular}




\section{$+34-$}

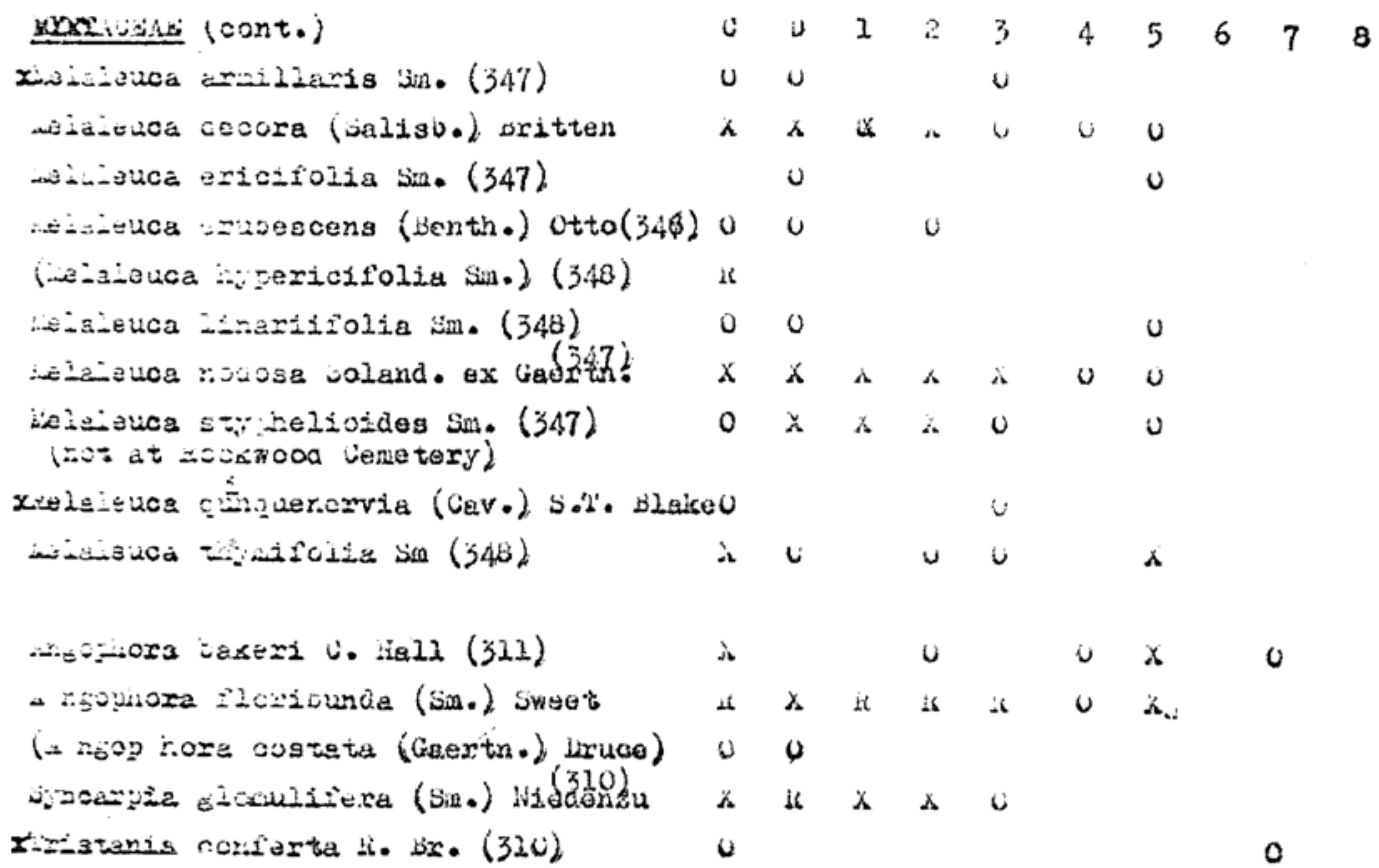

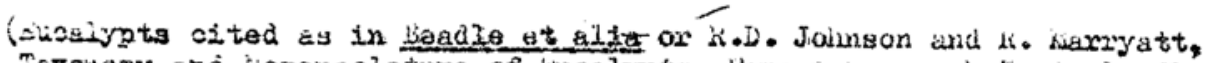

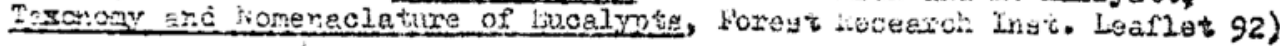
(usciyotus acacifformis leane et kaiden)(0) (0)

Whonirtus emplifolia Naudin (324)
0 is it
00

(-iceitrptus votryoides Simd

(u) (o)

(تuelyptus cinerea F. htill. ex Benth.

(ki) (at)

(aicilypus cladocalys F. Auell.)

(v) (k)

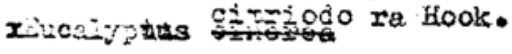

(Hcaiyptus ore exa F. Muell.) (318),

$x \quad(0)$

(iscelyptus exisia schau.) (334)

(B)

(Gucalyptis elate Dehnho) (327)

(ii)

$x$

0

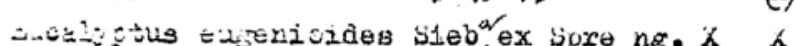

(3z)

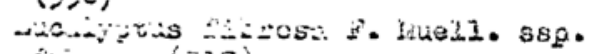

incosia $(51 ?)$

$x$ i $x$ x

inciontivs cloooinea blakely (351)

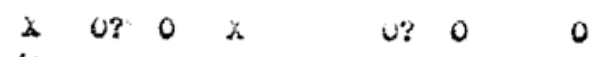

(-incilyptus globulus Labill. Bsp.

$(k)$ crovidus (-i)

Eincejytus Mooulus Labill. 38p. تtconil

art

(Eceigytus lobulus Labial. sop.

(0) (o)

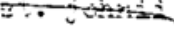

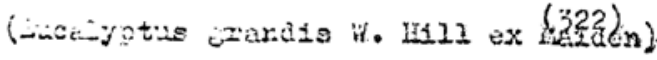

(c)

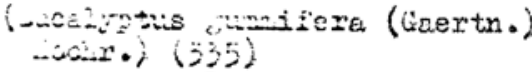

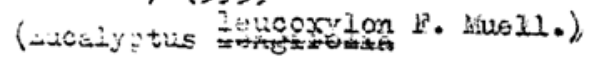

(is)

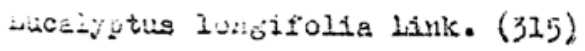

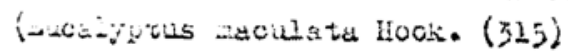

(a)

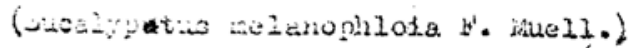

(x) (u)

$x$ x $x$ x 0

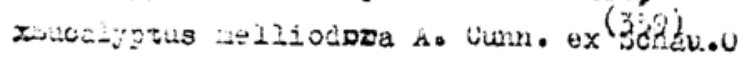

(iv)

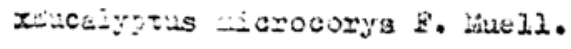

$x \quad x$

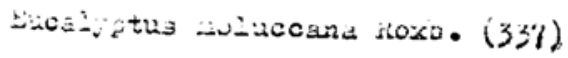

is $x<x$ 


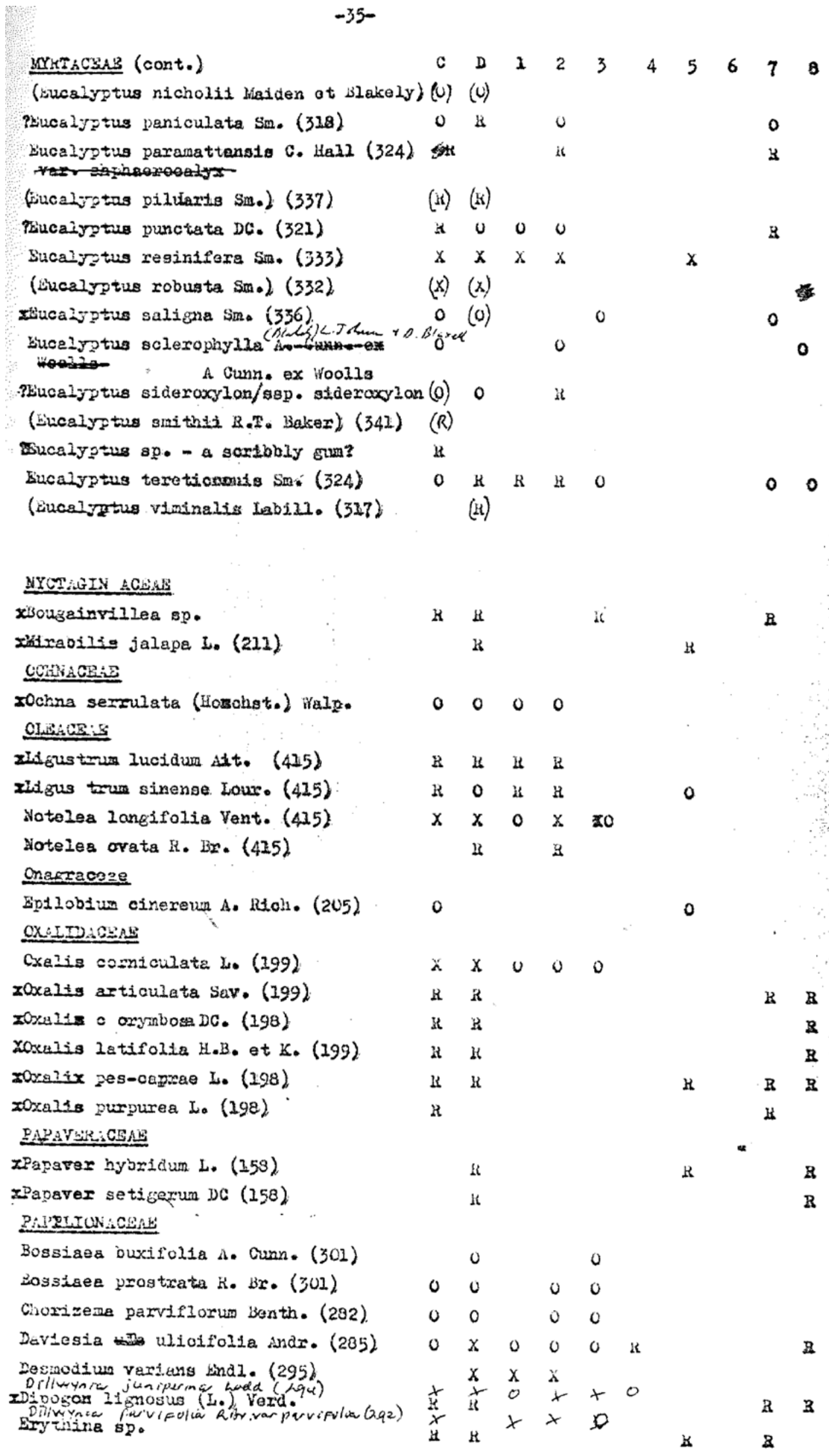




\begin{tabular}{|c|c|c|c|c|c|c|c|c|c|c|}
\hline$-30-$ & & & & & & & & & & \\
\hline 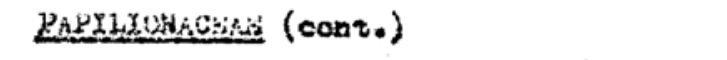 & c & 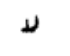 & 2 & 2 & 3 & 4 & 5 & 6 & 7 & $\mathbf{a}$ \\
\hline Elycine clandeatina andi. $(3 \omega)$ & $w$ & $s$ & $\therefore$ & $\therefore$ & $\Lambda$ & & & & & \\
\hline (1)ycine tapuciza benth. $(306)$ & $v$ & i & $u$ & v & $\checkmark$ & & & & & \\
\hline 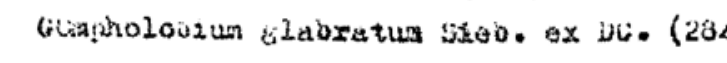 & & $v$ & & & 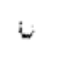 & & & & & \\
\hline 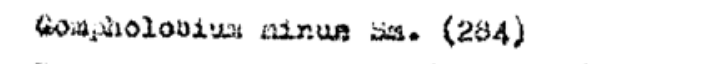 & $x$ & & & is & & & & & & \\
\hline 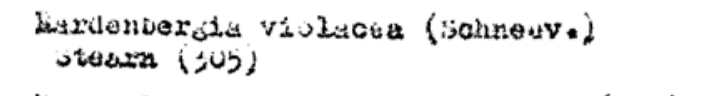 & i & $\lambda$ & $\therefore$ & a & $\therefore$ & ¿ & & & & 0 \\
\hline Hovea lanistiolite t. ix. ex ite (jwi) & & $v$ & & & & & 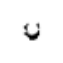 & & & \\
\hline 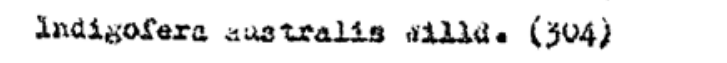 & & $y$ & $\dot{v}$ & c & 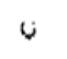 & & & & & 0 \\
\hline Jeckentia scokartu iteire. (294) & & s & is & & $\therefore$ & & & & & \\
\hline Xannedsa rubicinds kent. (sus) & $x$ & $\checkmark$ & & i & $\iota$ & $u$ & & & & \\
\hline xunimus ap. & is & & & & & & & & & घ \\
\hline 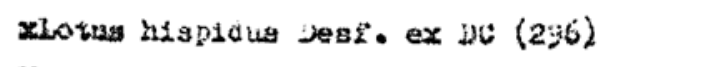 & $u$ & & & & i & & & & & \\
\hline XLotus angustisaiausi. $\left(290^{\circ}\right)$ & a & $x$ & & $\therefore$ & . & & & & & \\
\hline 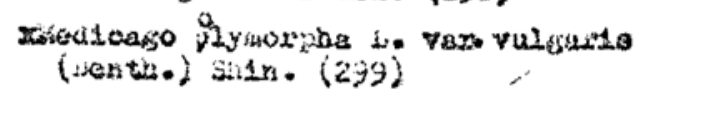 & $s x$ & 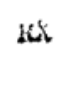 & & & is & & & & & $\underline{a x}$ \\
\hline Xedicaso sativa Le (299) & H & a & & & ii & & & & & 8 \\
\hline ibiolilotus inatos (L.) all. (290) & 0 & 0 & & & $i$ & & & & & 0 \\
\hline 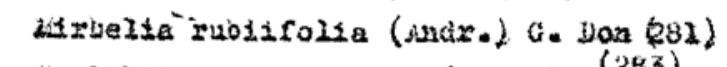 & is & & & & & & ה & & & \\
\hline 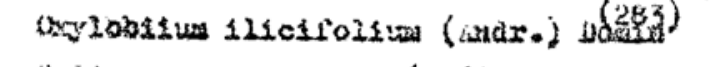 & 0 & & & $u$ & & & & & & \\
\hline 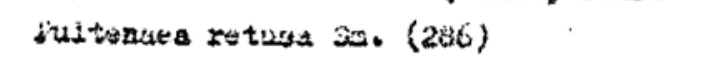 & is & is & & & si & & & & & $x$ \\
\hline 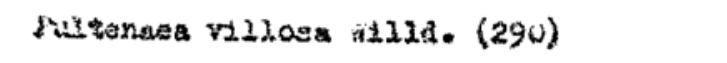 & 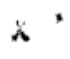 & $x$ & d & 0 & ن & $\lambda$ & & & 0 & 0 \\
\hline Jivavinla preudoacscia i. (304) & $x$ & & & & & & & & $x$ & \\
\hline 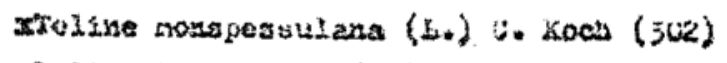 & $v$ & v & & & $\dot{s}$ & $v$ & & & & 0 \\
\hline Deline Lindrolin (L.) weve et berth & $u$ & & & & & & & & & 0 \\
\hline Xinsfolius exverse io $(29 a)$ & is & is & & & is & & & & & 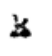 \\
\hline 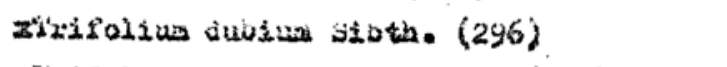 & $x$ & $x$ & & & $\check{a}$ & & $x$ & & & $x$ \\
\hline 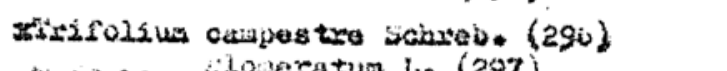 & $v$ & 0 & & & 0 & & 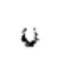 & & & 0 \\
\hline 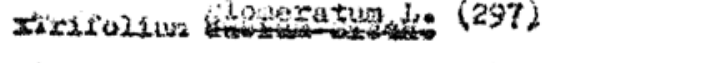 & $\ddot{x}$ & $\lambda$ & & & $\lambda$ & & $\ddot{z}$ & & & $x$ \\
\hline 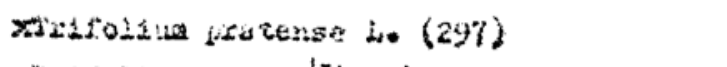 & $\therefore$ & K & & & $\therefore$ & & & & & a \\
\hline 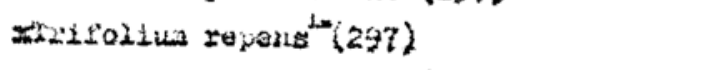 & $x$ & $\ddot{s}$ & & & $\therefore$ & - & $v$ & & & 0 \\
\hline 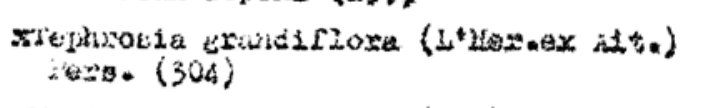 & u & & & Hh & & & ii & & & \\
\hline 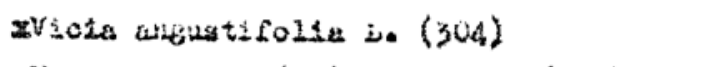 & $u$ & 0 & & & $u$ & & $s$ & & & 0 \\
\hline 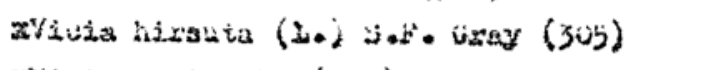 & $u$ & 0 & & & $i$ & & u & & & 0 \\
\hline siviclas eative Le $(j(4)$ & u & ن & & & 0 & & $\omega$ & & & 0 \\
\hline 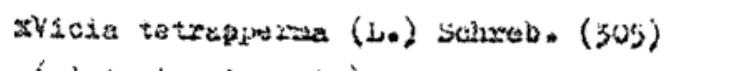 & 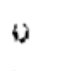 & 0 & & & $ن$ & & $v$ & & & \\
\hline 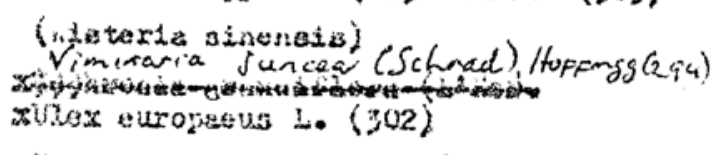 & 筇 & & & & & & $R$ & & 0 & \\
\hline Eorma dyct1ocanpa be. (295) & & is & & & $\therefore$ & & & & & \\
\hline \multicolumn{11}{|l|}{ 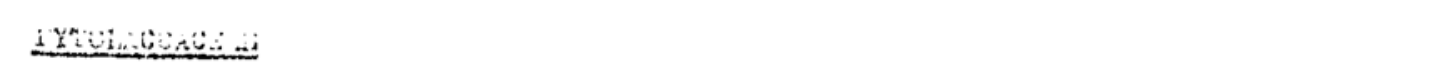 } \\
\hline 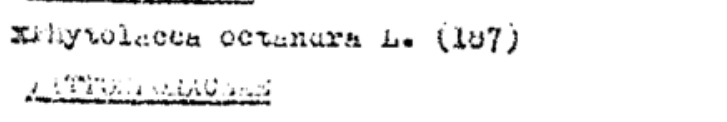 & 0 & $v$ & $v$ & $\mathrm{v}$ & & & 0 & & & \\
\hline 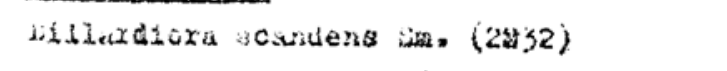 & it & 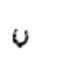 & & $u$ & & & & & & \\
\hline Lursuriat isinosa vav. $(252)$ & $\lambda$ & 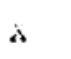 & $v$ & $x$ & $\iota$ & & & & & \\
\hline iztos)orwa dovolutua hato (252) & 0 & $s$ & v & 0 & & & & & & \\
\hline 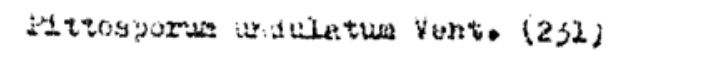 & i & $\omega$ & $u$ & 0 & & & & & & \\
\hline
\end{tabular}


$-3 i-$

\begin{tabular}{|c|c|c|c|c|c|c|c|c|c|}
\hline 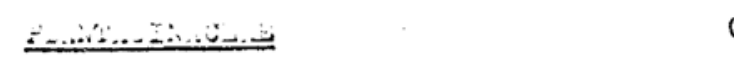 & c & b & 1 & 2 & 3 & 4 & i & 6 & 7 \\
\hline Xintaso coronopus L. $(433)$ & is & & & & & & k & & \\
\hline$x$-untajo -urceolata L. $(432)$ & 2 & $x$ & & & $\therefore$ & 0 & $x$ & & \\
\hline 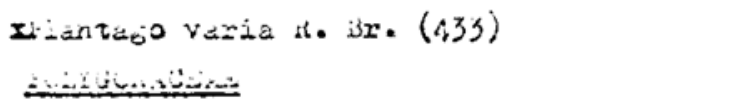 & & $x$ & & & $\lambda$ & & & & \\
\hline$x$ Liyooxir arenautrun & k & & & & & & is & & \\
\hline xojyzonum eviculare b. sens lat.(184) & 0 & 0 & & & & $u$ & & & \\
\hline Iolyzoram decipiens k. br. (185) & 0 & $x$ & & & & & $x$ & & \\
\hline Folyconia in iejathifolium L. (185) & K & r & & & & & $x$ & & \\
\hline Litex irowris usimpd. (183) & 0 & 0 & & & $v$ & & & & \\
\hline zivex crispus t. (104) & $x$ & $x$ & & & $\checkmark$ & & $x$ & & \\
\hline 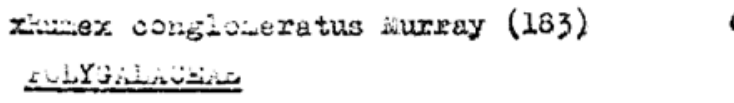 & 0 & 0 & & & $v$ & & $u$ & & \\
\hline xiroiysaia uyrtifolia b. (270) & st & & & & & & & & H \\
\hline 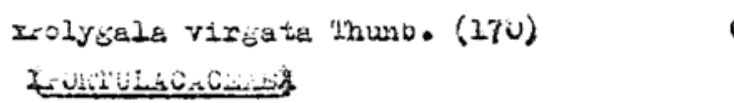 & $u$ & & & & & & $u$ & & 0 \\
\hline 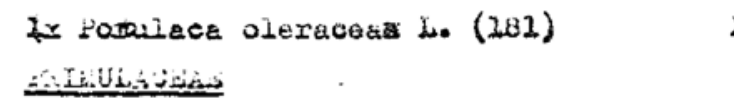 & $x$ & is & & & $\lambda$ & & & & $x$ \\
\hline 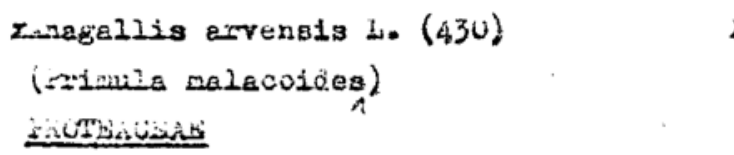 & $x$ & $\begin{array}{l}x \\
\text { is }\end{array}$ & & & & & z & & \\
\hline Enksia aspieniffolia Salisb. (220) & is & & & k & & & & & \\
\hline zenksis spinulosa Sil. (typ (218) & $x$ & & & $x$ & & & & & \\
\hline 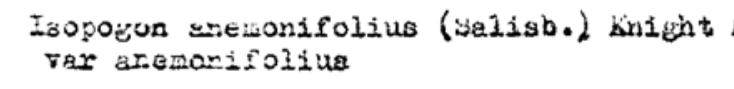 & k & & & s & & & & & \\
\hline Eersoonia lúurina Fers. & ß & & & 1 & & & & & \\
\hline Eersoania inearis And $x$ & & is & & & & & « & & \\
\hline 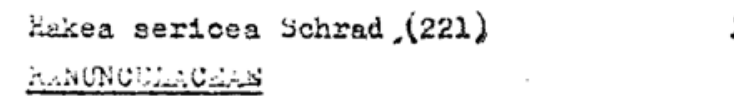 & $x$ & th & & 0 & & $x$ & is & & \\
\hline 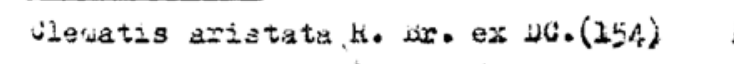 & a & 0 & is & 0 & & & & & \\
\hline Hematis olycinoider DC. (254) & & $u$ & $s$ & 0 & & & & & · \\
\hline 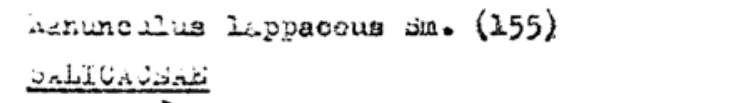 & & H & & « & & & k & & \\
\hline 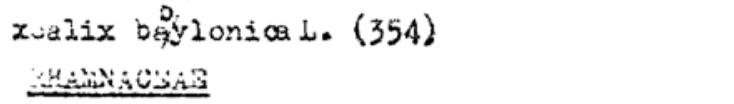 & & $u$ & & & & & 0 & & \\
\hline Fonaderzis ferruginea Sieb ex Lenzl. (36 & 6 (6) & $H$ & & 4 & & & $x$ & & \\
\hline Fonaderris lanifrera (A ndr.) Sirso (366) & & k & & & & & k & & \\
\hline 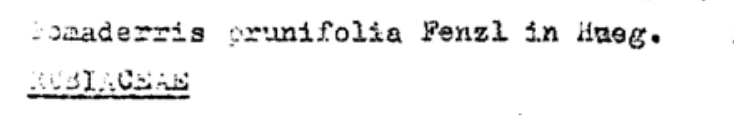 & $\mathrm{H}$ & & & H & & & & * & \\
\hline Vercularise aspexe linertn. (425) & 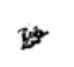 & 济 & 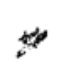 & 话 & $\Leftrightarrow$ & & & & \\
\hline Fercularis diphylue Gaertn. (425) & 0 & $x$ & 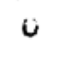 & $x$ & $u$ & & & & \\
\hline Garculeria varta took f. (425) & & k & & & & & is & & \\
\hline $\begin{array}{l}\text { ane velista (Gaertn.) Soland. ex } \\
\text { a lisch. }(424)\end{array}$ & $x$ & & $x$ & & & & & & \\
\hline 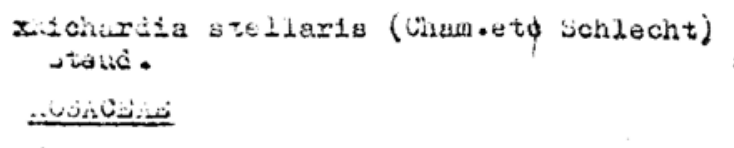 & 0 & 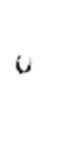 & & & $u$ & & & & \\
\hline $\begin{array}{l}\text { xiotoneaster sp. } \\
\text { xnotinia slabra }\end{array}$ & k & k & & $k$ & & & k & & \& \\
\hline
\end{tabular}




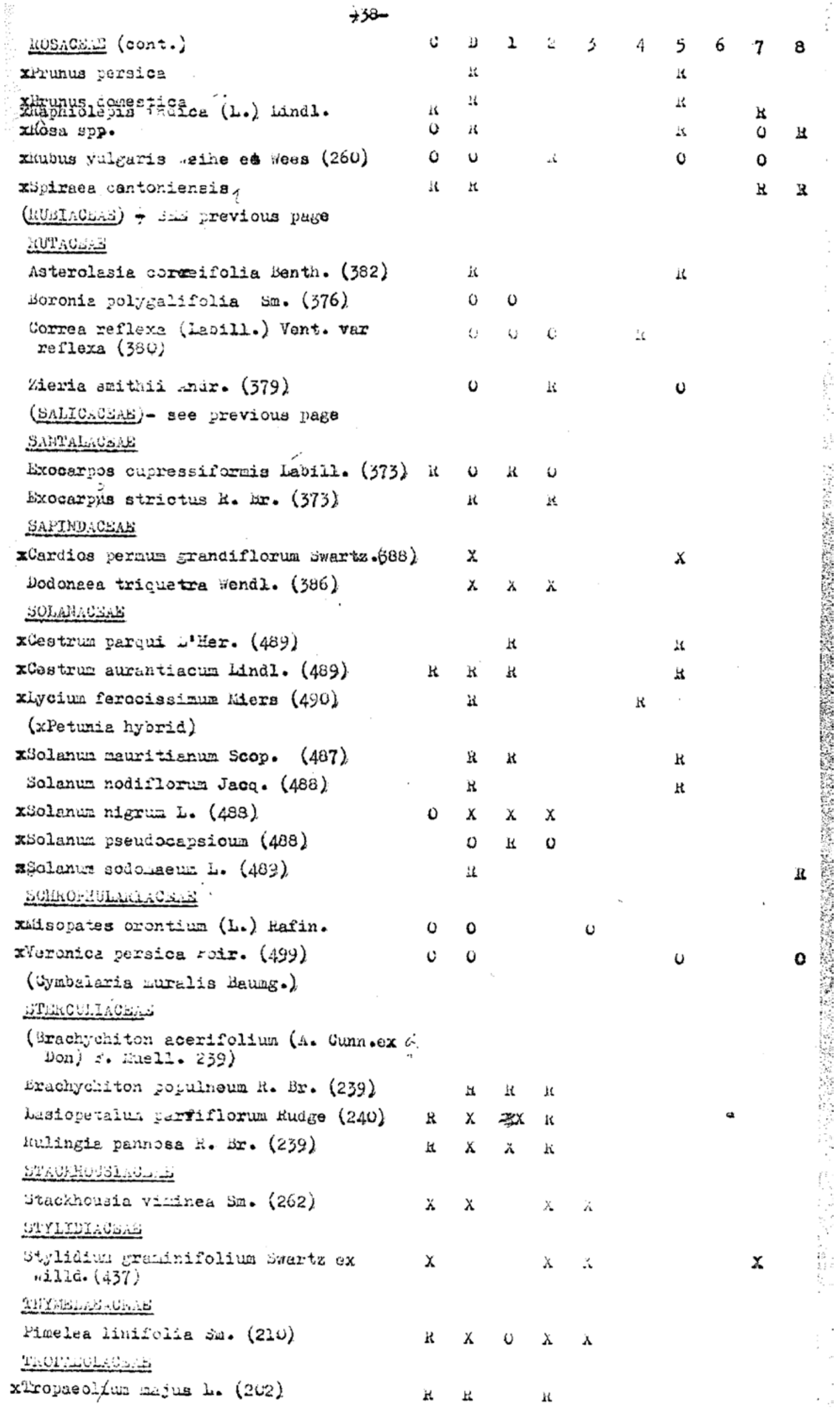




\section{UMASLIPSER}

xAptum zra veolens 1. (396)

C

xapiun leptoptyllum (Bers.) i. suell.

xpoenicuilu vuigare iatil.

0

xilycirocotyle Donarienais Leth. (394)

K $\mathrm{X}$

Platysece ezicoides (Siebo ex. jpreng.) $x$

c. ilozian

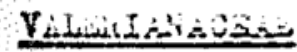

xCétrantives ruber DC (HIack IV BOI)

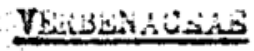

xhontans casora L.

$\begin{array}{lll}8 & 0 & 0\end{array}$

xbentana matevidensis (Jprenge) Lotą.

k

Wervane poxariensio I.

$x \quad x \quad 0 \quad 0$

Verbona officinalis t.

00

$\mathrm{VIulivij}$

viola betonsicifolia Sim. (168)

\section{SUIDYVISTO GOHOCUTYLEDONES}

\section{AGAVACP}

xheave azexicana I.

xighrinium cooitianum

B

k

$x$
xispittaris graninea wahx. var. veatherdiana (Hern.)Boerin (one lasisecoloxy)

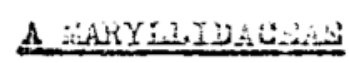

zallium neapolitianun Cyr.

'xarupantius orientelis

xcrinum peciuroulatin k. Br.

xavucojua estivum

rvarcissis (jonquilza?)

$(540)$

K

k k

$k$

w

is is

0

0

ofidpeastrum $x$ equestre (Dutch Hybrid).

C.

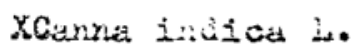

xerina 8 .

is

ti

k

HI

Andoxes

xintodeschis aethiopica (L.) ivreng.

$k$

k a

0

$x$

0

3

$x$

a

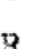

0

0

I

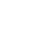

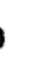

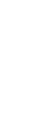




\section{$-39-$}

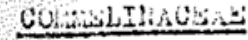

c $112 ; 4 ; 078$

xlradescantie aloillora kunth. (125)

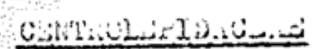

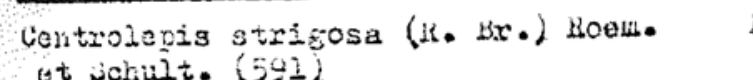

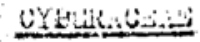

Garex inversa .. - 2 (617)

Gyathociseta diandra liees (60)

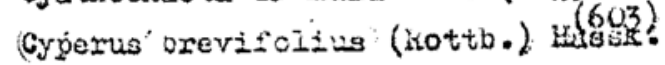

xiyperus aragrosis lam. (6ou)

oyperus airus o. clacke (bul)

Cyperus tenelius L. f. (6U1)

Lopidosporna leteraie R. br. (612)

lepicapering ineare k. Bx. (612)

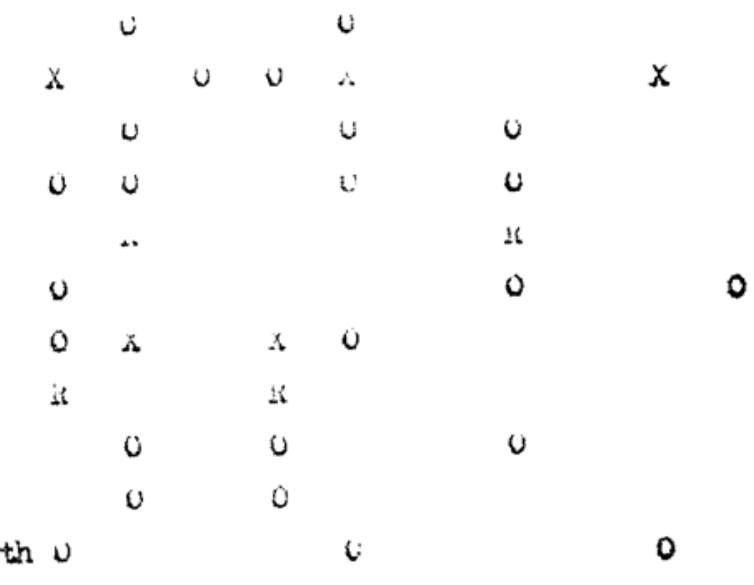

Guirita aspera (K. in.) Spreng. (6́14)

ithnia telarocarpa i. Bx. (614)

(

0

schoeius agoron kcem. et. Schult. (610) 0

xiscirius chlorostachys

18

Uesrpus invidatus (R.br.) Poir. 0

Xichrpus prolifers kotto. (606) 0

IYYOXIDAC

Hypoxis hycrosietrica Labizl. (552)

o $x$

k

o

0

GhuINizize (Poorly collected, Kookwood)

(Note that arrangement is not alphabetical but as in beadie et alia)

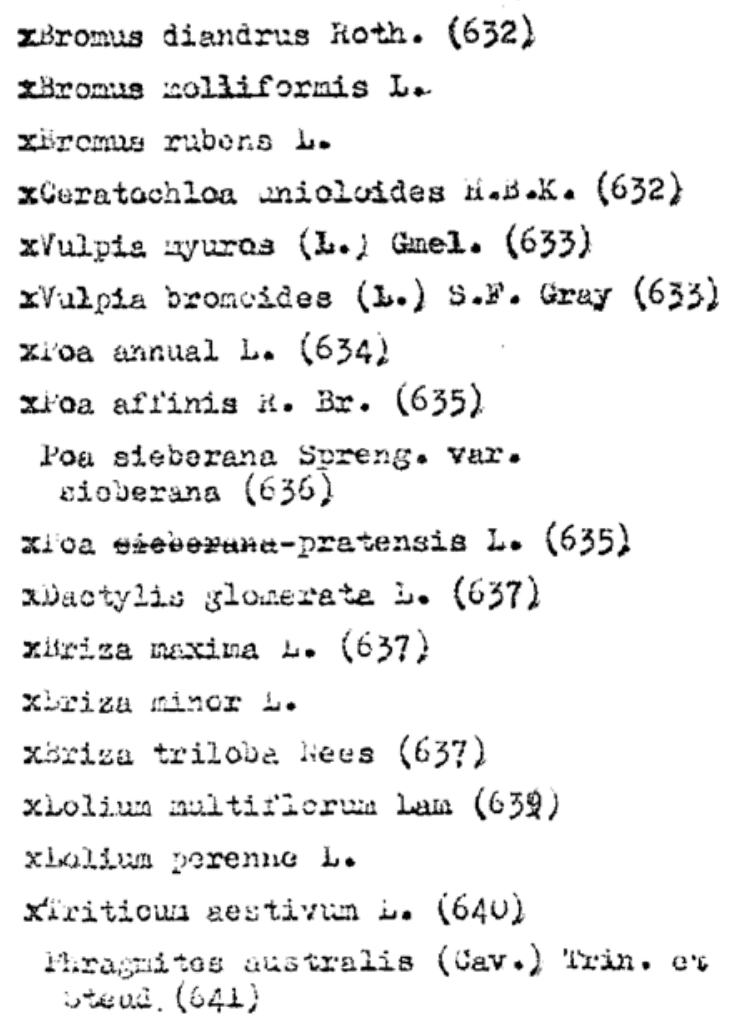

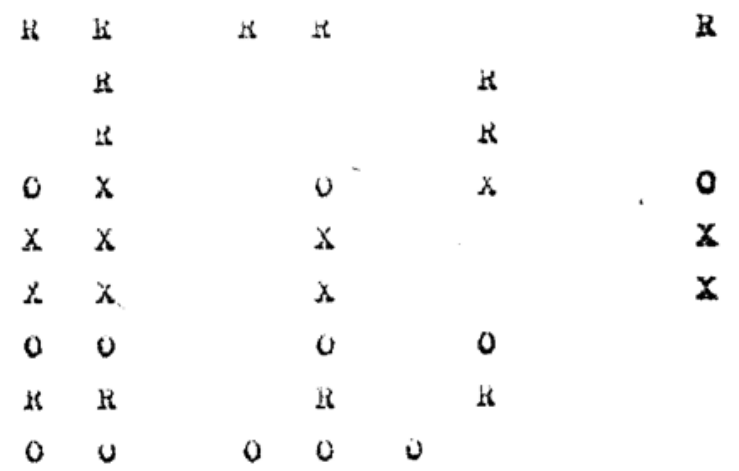

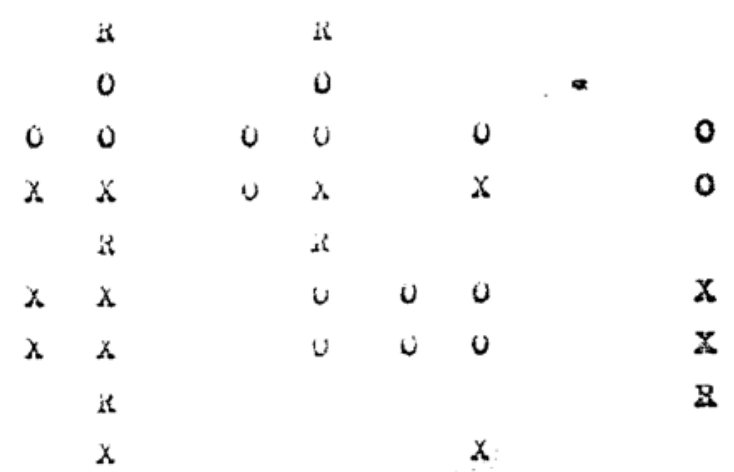




\begin{tabular}{|c|c|c|c|c|c|c|c|c|c|}
\hline Gesiritisine (cont.) & C & $\underline{\omega}$ & 2 & 2 & 3 & 4 & 5 & 6 & 7 \\
\hline $\begin{array}{l}\text { xCortateria selloana (Sohult.) A sohers } \\
\text { et iraoun. }(E<L)\end{array}$ & & si & & & & & H & & \\
\hline Lratgrostis arowili Nees ex bteud. (643) & 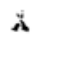 & $x$ & & & $i$ & $\dot{\Lambda}$ & & & \\
\hline 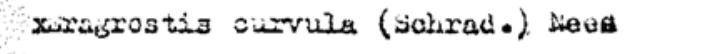 & & $\therefore$ & & & & & « & & \\
\hline Wragrostis iejtostachya iteud. (642) & & $u$ & & & $u$ & & & & \\
\hline Lragrostis 隹ipica Jedwabnick (642) & & $u$ & & & v & & & & \\
\hline 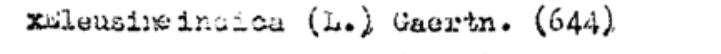 & & 0 & & & u & it & & & \\
\hline xilleusine tristacinja (Lem.)Lame & & $u$ & & & 0 & & & & \\
\hline $\begin{array}{l}\text { Xiporolobus afutcanus (koir.) kobyns } \theta t \\
\text { Tournay }(6<5)\end{array}$ & $x$ & $\therefore$ & & & & $\hat{\lambda}$ & & & \\
\hline Sporolozus eiongatis K. is. (645) & ن & u & & & $\mathbf{C}$ & & & & \\
\hline 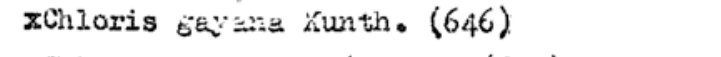 & & h & & & ii & & & & \\
\hline Gharis truceata fik. isr. (645) & & $x$ & & & $x$ & & $\dot{\boldsymbol{\lambda}}$ & & \\
\hline$\pi$ (hloris vizosina jwartz $(646)$ & & 13 & & & & & & & \\
\hline xoynucion dactsion (h.l pers. $(646)$ & $x$ & $x$ & & & $x$ & $x$ & $x$ & & \\
\hline xulvena barbeza scot. $(647)$ & $x$ & $x$ & & & 6 & & $x$ & & \\
\hline xivena fatie Le & u & & & & & & & & \\
\hline xavena ludoviatana bux. & K & is & & & & & & & \\
\hline xivena stemis is to $(647)$ & k & & & & & & & & \\
\hline xiolcus lanatis $\mathrm{I} .(648)$ & $\mathrm{K}$ & & & & & & $\mathrm{K}$ & & \\
\hline Anghibromus netsis steud. (648) & & is & & & & & $\mathrm{k}$ & & \\
\hline 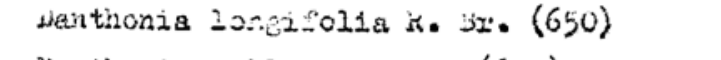 & & it & & & & & & & \\
\hline Wanthonia peiiida A. Bx. (649) & $\mathbf{k}$ & & & & & & K & & \\
\hline Denthonia Fupurascens Jo Vickery $(551)$ & $x$ & $\mathrm{x}$ & & 0 & C & $x$ & & & \\
\hline Wantihonia racesosak. Hr. $(651)$ & & ז & & $\mathrm{H}$ & & & & & \\
\hline Denthorta 2 eteces H. 3x. $(650)$ & & 0 & & & 0 & & & & \\
\hline A Grostis cezula K. Br. $(652)$ & $x$ & $x$ & & 0 & $x$ & & $x$ & & \\
\hline Arrostis avenacea limel. (652) & $x$ & $x$ & & 0 & $x$ & & $x$ & & \\
\hline Deyeuxis quecriseta (bioill.) bento & $x$ & $\mathrm{x}$ & & 0 & $x$ & & & & \\
\hline Echinopogon Jvetus (gorst. 20) Begur. & $\dot{x}$ & $x$ & i & $\dot{A}$ & & & & & \\
\hline Eohinonogon caespitosue C.E. Hubserd & $x$ & $x$ & $x$ & $x$ & & & & & . \\
\hline jolutacine sciurea (Hoor.) Hook 955$)$ & $x$ & $x$ & u & 0 & $x$ & u & $x$ & & \\
\hline Wicholachne =era (k. Bro) J Hiokery & & is & & & $\mathfrak{k}$ & & & & \\
\hline xilny ojon congpeliansis (L.)Lesf. $(655)$ & K & u & & & & & & $\mathfrak{H}$ & \\
\hline Sttpa nolisis $\mathrm{x} \cdot \mathrm{B2} \cdot(656)$ & $\mathrm{k}$ & & & & $\mathfrak{k}$ & & & & \\
\hline itipa neryuse j. Vickery ver. nervoea & $x$ & $x$ & it & $x$ & $x$ & & & & \\
\hline Stipa pubeveens Ko Br. (657) & $x$ & $x$ & & $\mathrm{x}$ & $u$ & & & & \\
\hline Stipa scibre Iind2. & & 0 & & 0 & 0 & & & & \\
\hline arigtica rstose K. Bxo(658) & & k & & & u & & & 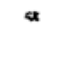 & \\
\hline aristide vecens filav. (65.28) & $x$ & 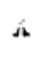 & & $i$ & $\Delta$ & & & & \\
\hline xhaleris ticenosa L. (661) & & 0 & & & U & & & & \\
\hline 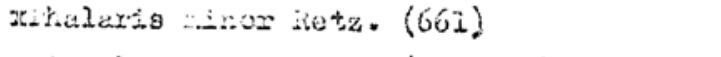 & & 0 & & & $v$ & & & & \\
\hline 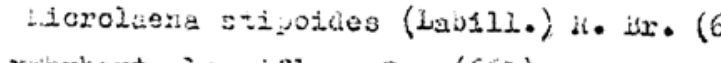 & 6011) & $\check{n}$ & L. & $\dot{\lambda}$ & $\alpha$ & & $x$ & & \\
\hline 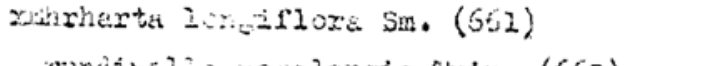 & & $i$ & & & & is & & & \\
\hline .rundíleliz vejalengis 2rin. (662) & k & & & & & & $\pi$ & & \\
\hline 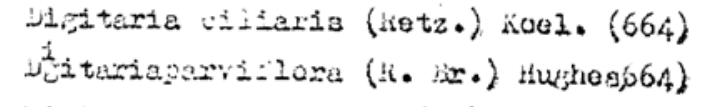 & & $u$ & & $u$ & $u$ & & $u$ & & \\
\hline 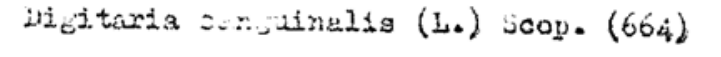 & & 0 & & & $u$ & & & & \\
\hline
\end{tabular}




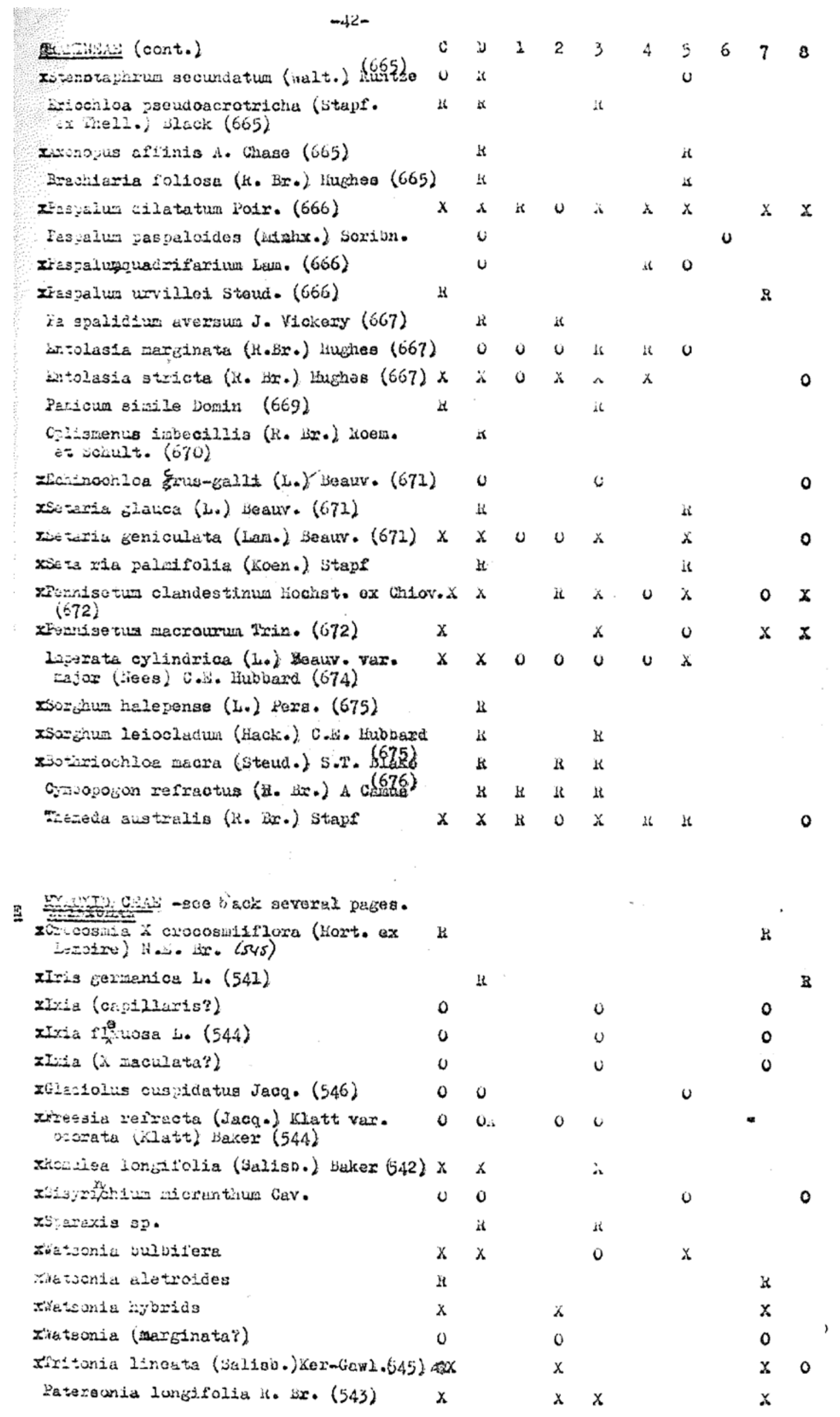




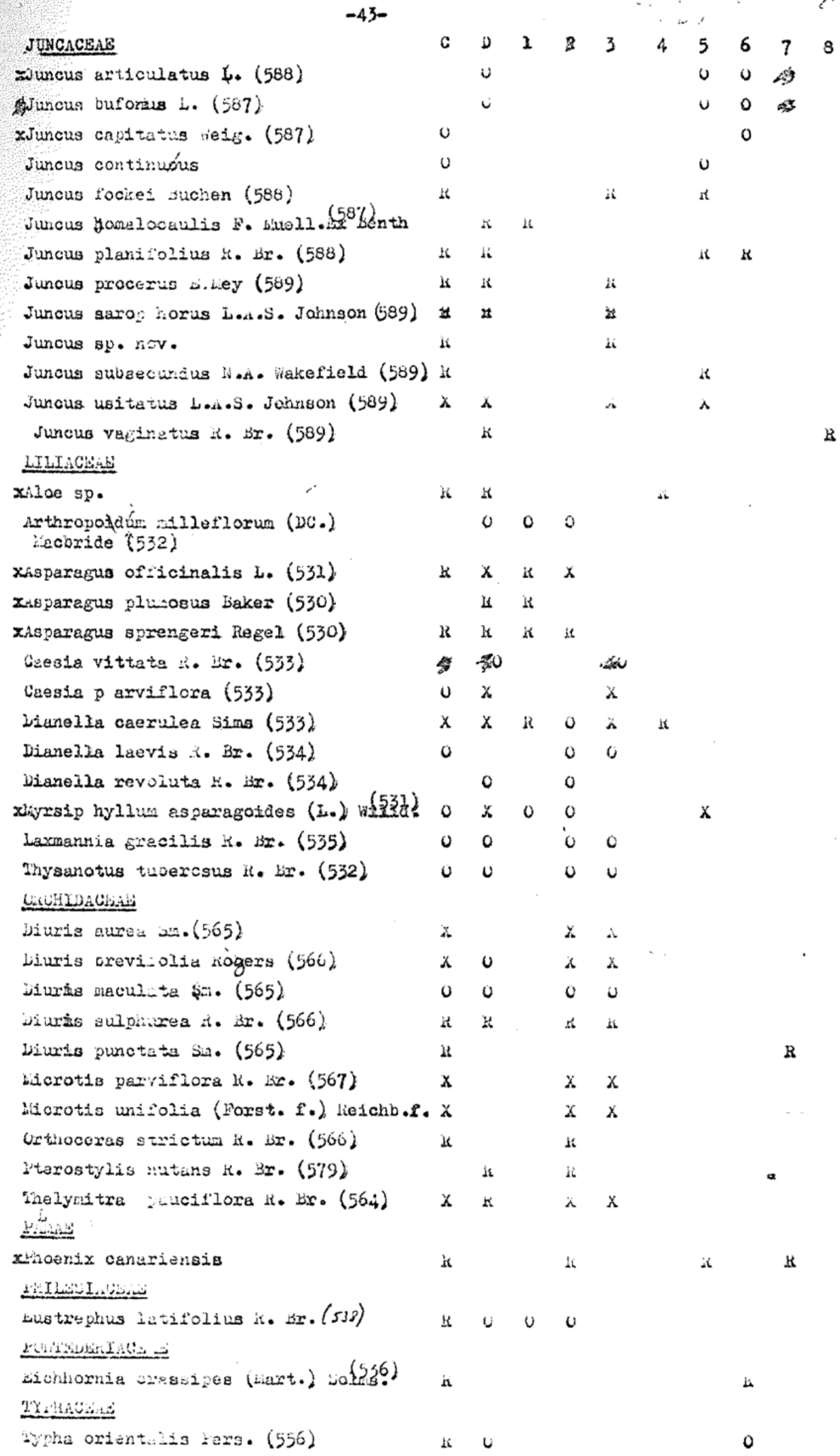


$-44-$

\begin{tabular}{|c|c|c|c|c|c|c|c|c|c|}
\hline 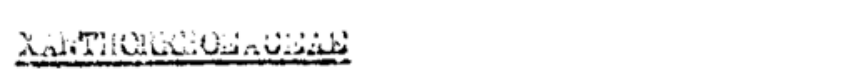 & C & ע. & 2 & 2 & 3 & $4_{4}$ & 5 & 6 & 7 \\
\hline Wountra cylincrica dee (549) & c & & & & $u$ & & & & \\
\hline 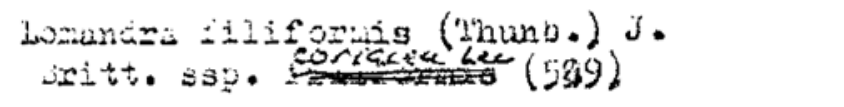 & $v$ & $u$ & & i & U & & & & \\
\hline Lotiandas lluriatilis (k. jro) Loo (550) & & 0 & & & 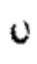 & & & & \\
\hline 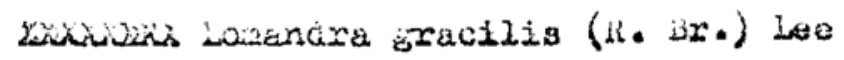 & $x$ & & & & $\lambda$ & & & & \\
\hline Lovenda & & $v$ & & u & & & & & \\
\hline 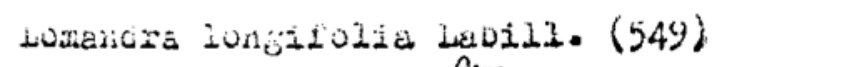 & $u$ & $v$ & & $v$ & & & & & \\
\hline $\begin{array}{l}\text { Xinthorrinoea resinosa pers. } \\
\text { concave les } \\
\text { (547) }\end{array}$ & u & $v$ & di & $u$ & $u$ & & $u$ & & 0 \\
\hline
\end{tabular}




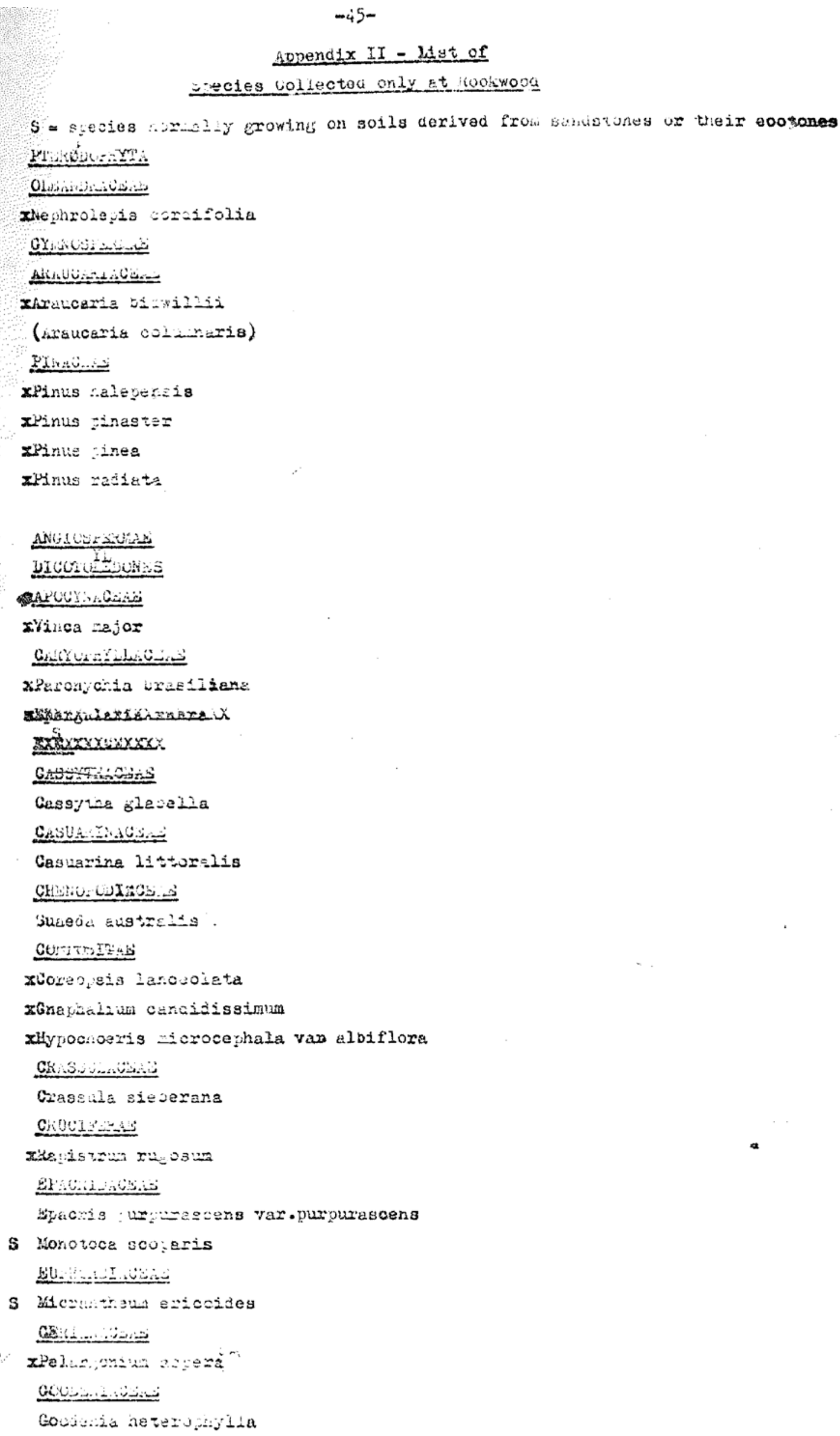




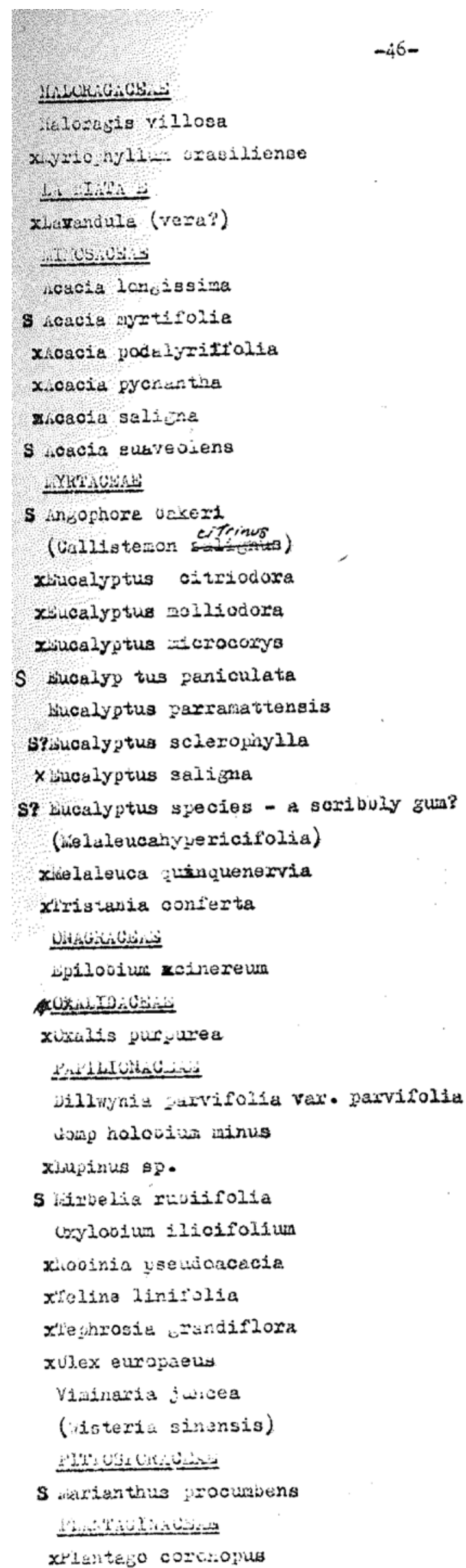

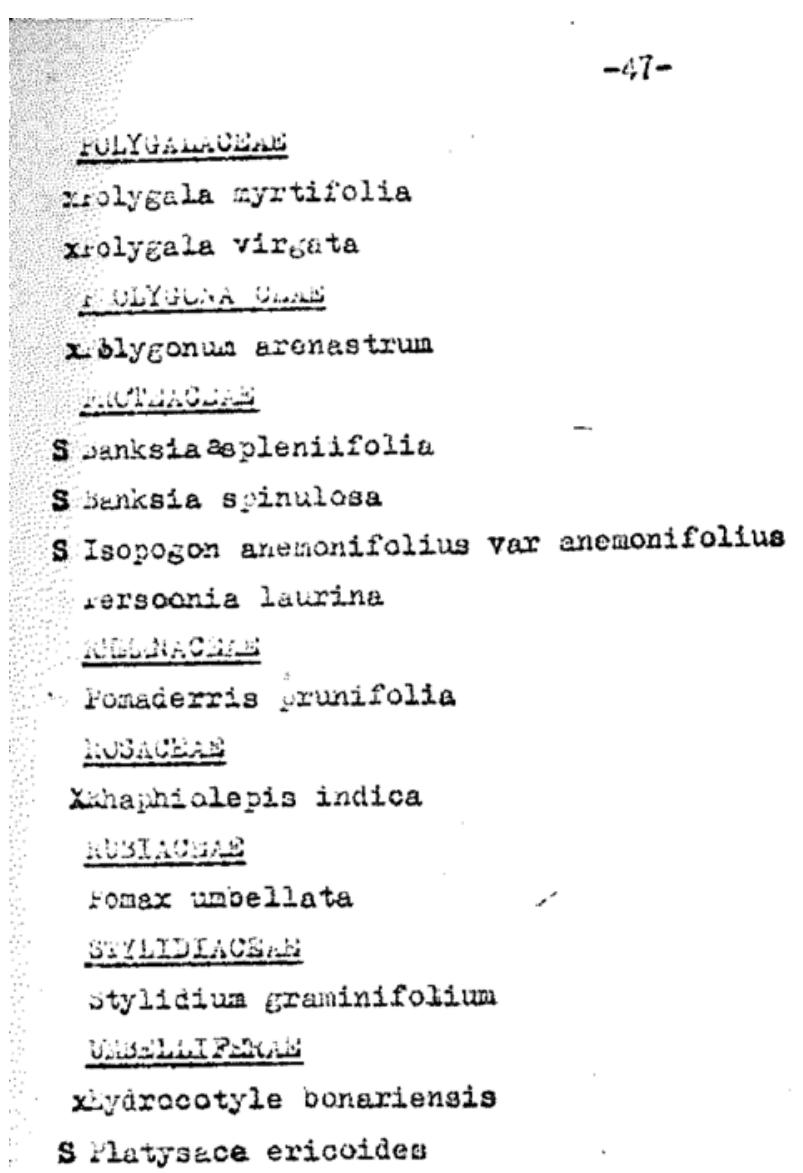

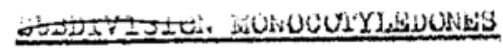

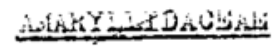

xillium neapolitionum

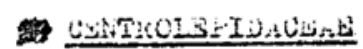

Cs? Centrolefis strigoga

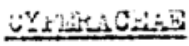

s Gyathocianete diandra oyperus tenallus

t S? Loj izosperwa linaare

(s) ichoenus aposon

s itizantheliua devotum xiciryus chlorostachys نcirpus inundatus icirgus rolifer 证

x.vena sterilis

x.olcus lenatus Janthonia pillida itije collis rundinejla nepalensis

$x$ rogatum urrillei suicus sicile x-entisetwe horoun 


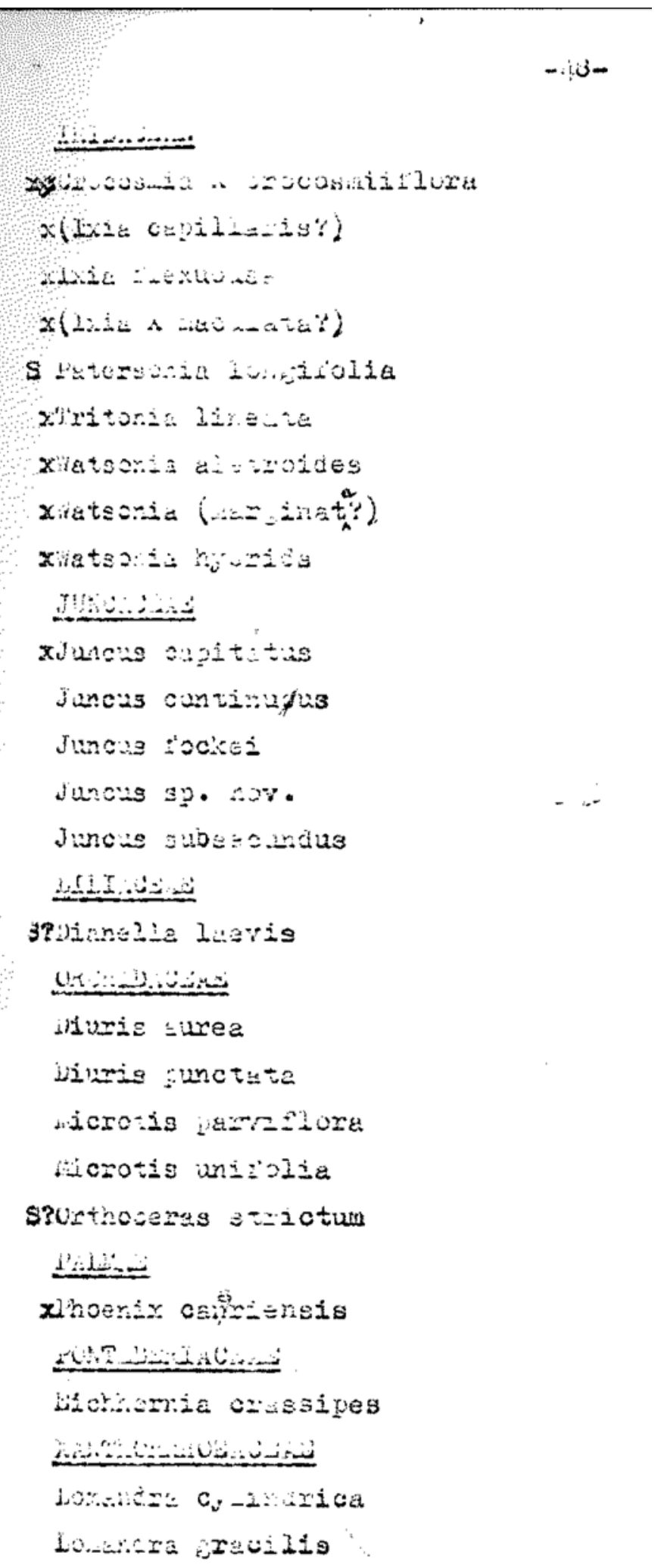




\section{nibliogrengy}

hitos

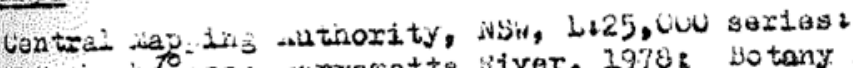

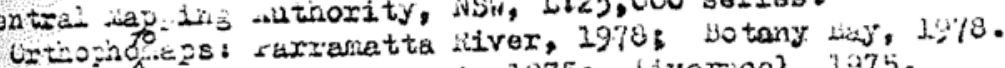

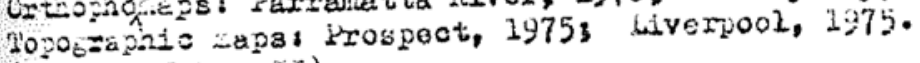

(Jource or hep II).

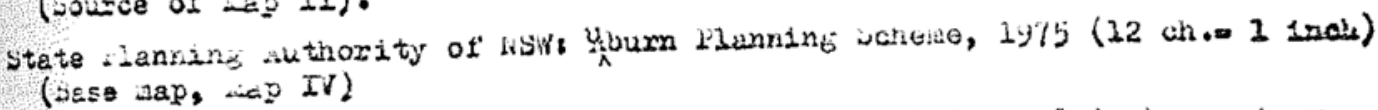

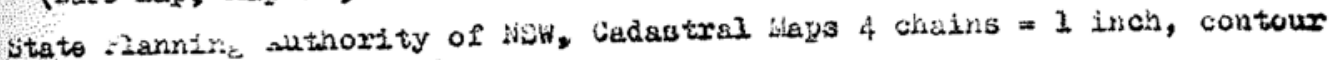

interral lcit. : iruck uiver area

(ذese sip, ip III)

Auburn junicizal council, Cadastrel hapa 2 chaing $=1$ inch, contour interras.

5ft. Canarion Gojf Courve, State Hosptital and lookwood venetery urea

(Popozraphíc detisiz, hap IV)

Valuer jenereisisepartment, Regional Offlce Rexraniatia; Vutastual waps

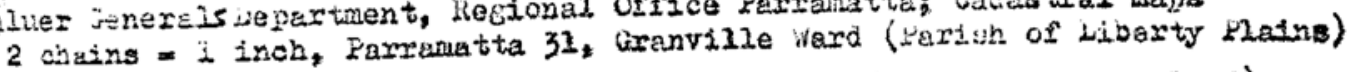

Lands jepartient of lvSil, Paxish of biberty Plating (vounty of wumoerland), 3ra ejiticn (1202), 4 the and 5 the Gditions.

Lianuscript sources

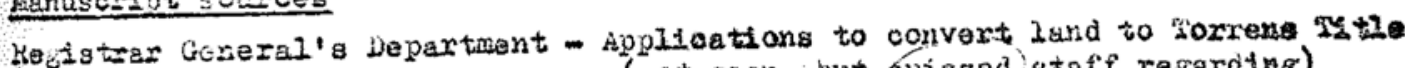

than! hat heri!!

archives of .. Sh

bichell hioeary

Articles Charies 1 Bertie

c. Leriert

A.I. Dee

A.l. LeS

bitzaveth bogex

L.s.it. johnson

joyce, viciery

Joyce te $\sqrt{\text { icjesy }}$

See ofs pso.

Booirs

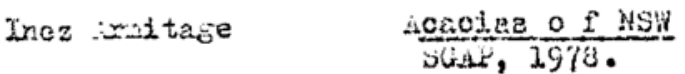

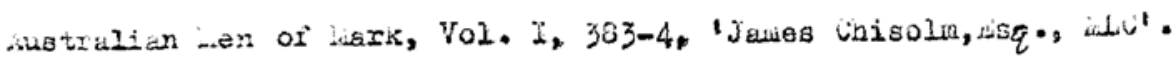

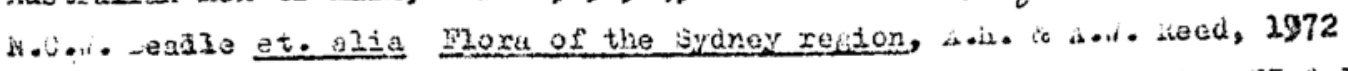

.H.C... . joajle

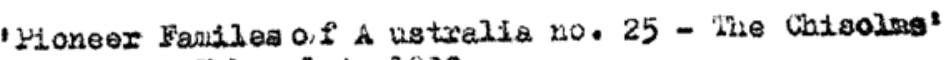
The Home, Lbby. 13t.,1932.

ine sedimentology and Palaeoenvirurment of the Iriass io Vianamatta Group of Sancistones, Sydney Basin?

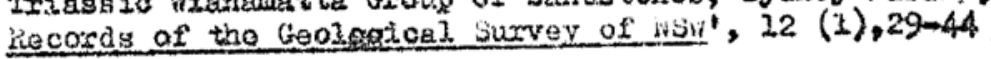

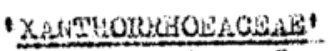
Contrivutiong From the NSir Nationel llaxpariun, sore series, no. 34.

Notes on Kanthorxhoes in Lestorn is ustruitia, Contrioutions from the wibl Hational Herbariun, Iv (1) $35 \mathrm{fs}$

The loufleas spectes of JUhivis in Hew Lealand, Naw wealand $J$. of bat., II, 1964, 277ff.

Now vyecies o fJunous in Australica and Wew Lealand, 2Azes.

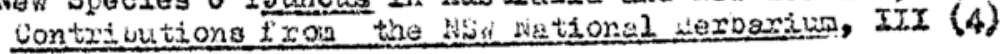

A revision of the A ustrejian jpeoieg of Denthonis DC., c rrom the HSil Nat. Lerp., 21 (3) 24 lift.

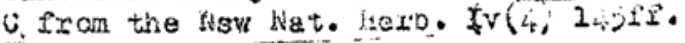

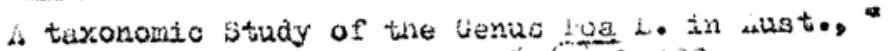




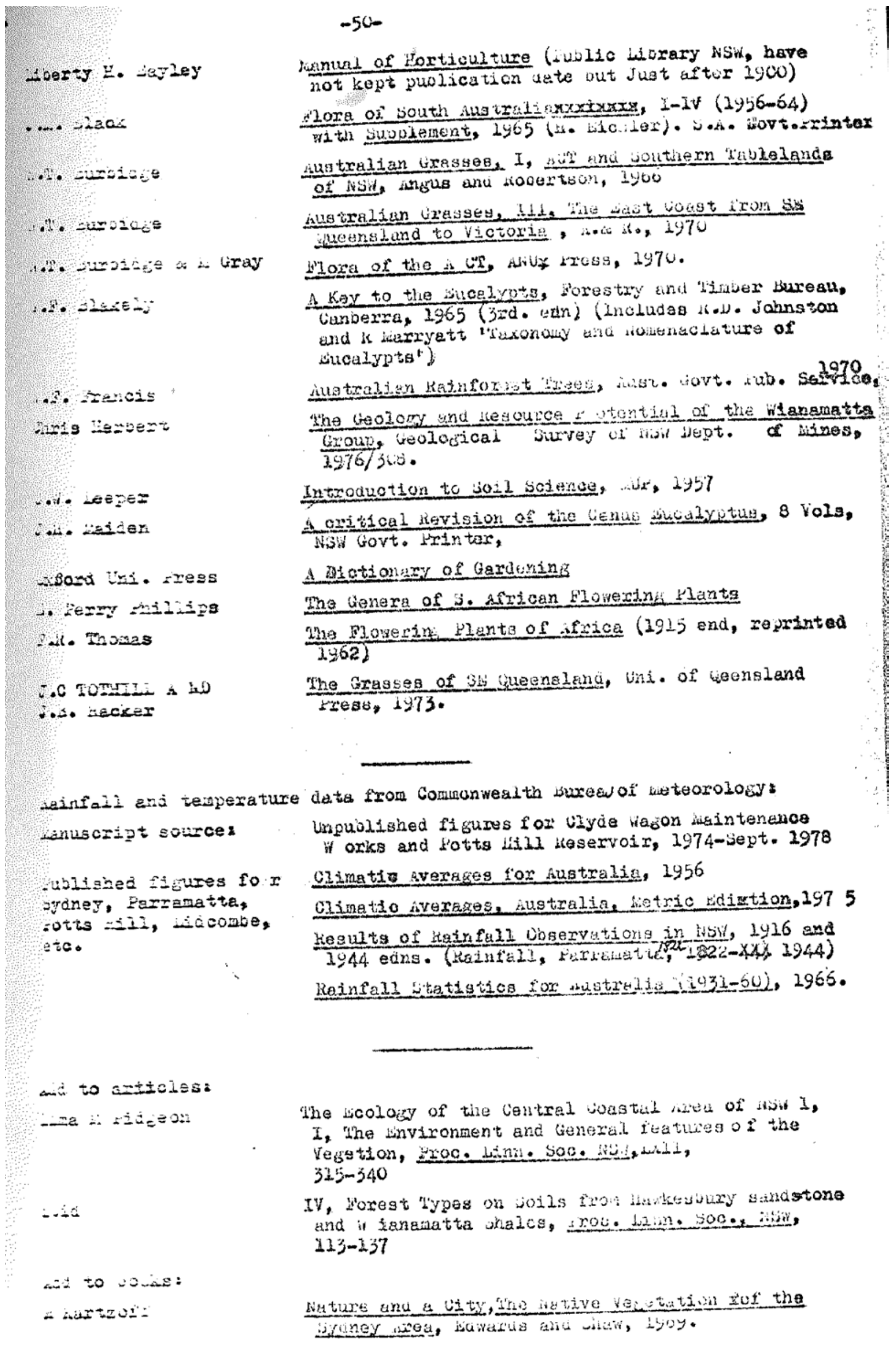

